Florida International University FIU Digital Commons

\title{
The Effect of Hyperthermia on Doxorubicin Therapy and Nanoparticle Penetration in Multicellular Ovarian Cancer Spheroids
}

Abhignyan Nagesetti

Florida International University, anage001@fiu.edu

DOI: 10.25148 /etd.FIDC001791

Follow this and additional works at: https://digitalcommons.fiu.edu/etd

Part of the Bioimaging and Biomedical Optics Commons, Biological Engineering Commons, $\underline{\text { Biomaterials Commons, Biomechanics and Biotransport Commons, Molecular, Cellular, and Tissue }}$ Engineering Commons, and the Other Biomedical Engineering and Bioengineering Commons

\section{Recommended Citation}

Nagesetti, Abhignyan, "The Effect of Hyperthermia on Doxorubicin Therapy and Nanoparticle Penetration in Multicellular Ovarian Cancer Spheroids" (2017). FIU Electronic Theses and Dissertations. 3183.

https://digitalcommons.fiu.edu/etd/3183 


\title{
FLORIDA INTERNATIONAL UNIVERSITY
}

Miami, Florida

\section{THE EFFECT OF HYPERTHERMIA ON DOXORUBICIN THERAPY AND NANOPARTICLE PENETRATION IN MULTICELLULAR OVARIAN CANCER SPHEROIDS}

\author{
A dissertation submitted in partial fulfillment of \\ the requirements for the degree of
}

DOCTOR OF PHILOSOPHY

in

BIOMEDICAL ENGINEERING

by

Abhignyan Nagesetti

2017 
To: $\quad$ Interim Dean Ranu Jung

College of Engineering and Computing

This dissertation, written by Abhignyan Nagesetti, and entitled The Effect of Hyperthermia on Doxorubicin Therapy and Nanoparticle Penetration in Multicellular Ovarian Cancer Spheroids, having been approved in respect to style and intellectual content, is referred to you for judgment.

We have read this dissertation and recommend that it be approved.

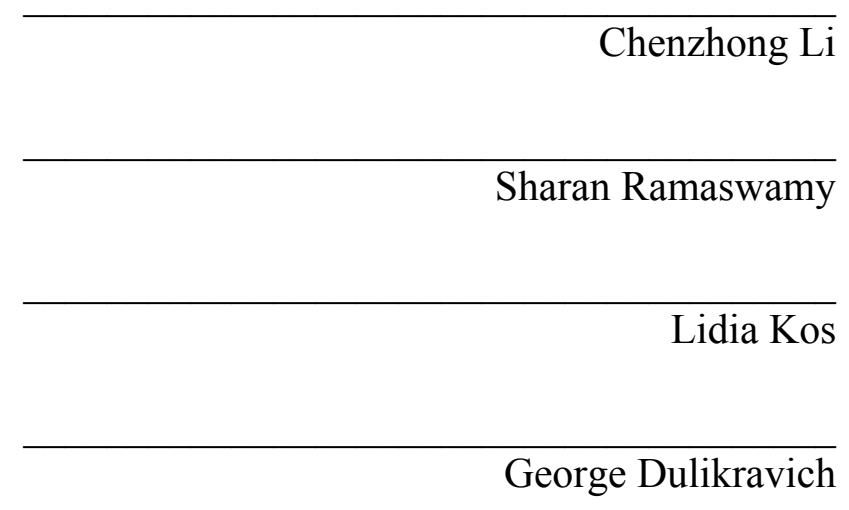

Anthony J.McGoron, Major Professor

Date of Defense: February 12, 2017

The dissertation of Abhignyan Nagesetti is approved.

Interim Dean Ranu Jung

College of Engineering and Computing

Andrés G. Gil

Vice President for Research and Economic Development and Dean of the University Graduate School

Florida International University, 2017 
(C) Copyright 2017 by Abhignyan Nagesetti

All rights reserved. 


\section{DEDICATION}

This work is dedicated to my maternal grandmother, the Late Vatrapu

Subbalakshmi Reddy. Her positive spirit always soared above her sickness. She raised me during the formative years of my childhood when my parents were working, but left me too soon before I was able to take care of her. 


\section{ACKNOWLEDGMENTS}

I thank my Amma (Mother), Sandhya Kumari, with all my heart. In my opinion, she has given me the greatest gifts that a parent can give to his/her child, trust and freedom. My nannagaru (Father) Nagesetti Madhusudhan Rao for being patient and kind. To my sister who is still trying to find her place in this world, I hope I will be the brother she can look up to. I promise I will always be there to support her in whatever direction she chooses to take.

I would like to give my deepest gratitude to Shri. Dr Anthony J McGoron for his unflinching commitment to further independent research of his students. He has instilled in me the core skills of attention to detail and critical thinking necessary to undertake high quality scientific research. Dr Romila Manchanda for teaching me emulsion chemistry that was later critical for preparation of colloidal theranostic probes. The committee members, Dr. Lidia Kos, Dr. Chenzhong Li, Dr. Sharan Ramaswamy and Dr. George Dulikravich for being patient and accommodating to my requests. Their advice improved the quality of my research. Dr. George Dulikravich was instrumental in developing the inverse estimation technique to model cellular uptake of nanoparticles under hyperthermia. Dr. Dulikravich's students Diego Cardoso Estumano wrote the preliminary code for the Monte Carlo method and Dr. Abas Abdoli provided ideas for the finite element model. My labmates, Dr. Supriya Srinivasan, Dr. Tingjun Lei, Dr. Yuan Tang, Dr. Vinay Bharadwaj and Dr. Alicia Fernandez-Fernandez whose achievements I will always look up to.

To all of my friends, who are too many to list and I will refrain from doing so for the fear of omitting any names. They are the most important, wonderful and amazing 
people in my life. They have strengthened my faith in love and kindness. The little ones that they brought into this world fill me with hope and gusto to work towards creating a better future.

For Office of Global Learning Initiatives (OGLI), especially Shri Dr. Hilary Landorf, for providing me financial support and helping a graduate student like me in the best possible way. I am forever grateful for the confidence and support she has shown in me. The OGLI staff, Dr. Stephanie Doscher, Melissa Livingston, soon to be PhD's Eric Feldman, Bahia Simons-Lane and Dr Bimal Soti. The work that they do is incredibly nuanced and very cool. They will be my inspiration for making the world a better place through education.

Last but not the least, I would like to thank the Department of Biomedical Engineering for providing financial support in the initial stages of my graduate studies. Ms. Claudia Estrada for just being very nice. To all the undergraduate students that I mentored and helped me with my work. The Minority Biomedical Research Initiatives for providing me with a summer research grant in 2014. 


\begin{abstract}
OF THE DISSERTATION
THE EFFECT OF HYPERTHERMIA ON DOXORUBICIN THERAPY AND

NANOPARTICLE PENETRATION IN MULTICELLULAR OVARIAN CANCER

SPHEROIDS
\end{abstract}

by

Abhignyan Nagesetti

Florida International University, 2017

Miami, Florida

Professor Anthony J. McGoron, Major Professor

The efficient treatment of cancer with chemotherapy is challenged by the limited penetration of drugs into the tumor. Nanoparticles $(10-100$ nanometers) have emerged as a logical choice to specifically deliver chemotherapeutics to tumors, however, their transport into the tumor is also impeded owing to their bigger size compared to free drug moieties. Currently, monolayer cell cultures, as models for drug testing, cannot recapitulate the structural and functional complexity of in-vivo tumors. Furthermore, strategies to improve drug distribution in tumor tissues are also required. In this study, we hypothesized that hyperthermia $\left(43^{\circ} \mathrm{C}\right)$ will improve the distribution of silica nanoparticles in threedimensional multicellular tumor spheroids. Tumor spheroids mimic the functional and histomorphological complexity of in-vivo avascular tumors and are therefore valuable tools to study drug distribution. Ovarian cancer (Skov3) and uterine sarcoma (MESSA/Dx5) spheroids were generated using the liquid overlay method. The growth ratio and 
cytotoxicity assays showed that the application of adjuvant hyperthermia with Doxorubicin (DOX) did not yield higher cell killing compared to DOX therapy alone. These results illustrated the role of spheroids in resistance to heat and DOX. In order to study the cellular uptake kinetics of nanoparticles under hyperthermia conditions, the experimental measurements of silica nanoparticle uptake by cells were fitted using a novel inverse estimation method based on Bayesian estimation. This was coupled with advection reaction transport to model nanoparticle transport in spheroids. The model predicted an increase in Area Under the Curve (AUC) and penetration distance $\left(\mathrm{W}_{1 / 2}\right)$ that were validated with invitro experiments in spheroids. Based on these observations, a novel multifunctional theranostic nanoparticle probe was created for generating highly localized hyperthermia by encapsulating a Near Infrared (NIR) dye, IR820 (for imaging and hyperthermia) and DOX in Organically modified silica nanoparticles (Ormosil). Pegylated Ormosil nanoparticles had an average diameter of $58.2 \pm 3.1 \mathrm{~nm}$, zeta potential of $-6.9 \pm 0.1 \mathrm{mV}$ and high colloidal stability in physiological buffers. Exposure of the IR820 within the nanoparticles to NIR laser led to the generation of hyperthermia as well as release of DOX which translated to higher cell killing in Skov3 cells, deeper penetration of DOX into spheroids and complete destruction of the spheroids. In-vivo bio-distribution studies showed higher fluorescence from organs and increased plasma elimination life of IR820 compared to free IR820. However, possible aggregation of particles on laser exposure and accumulation in lungs still remain a concern. 


\section{TABLE OF CONTENTS}

CHAPTER

PAGE

Introduction.

1

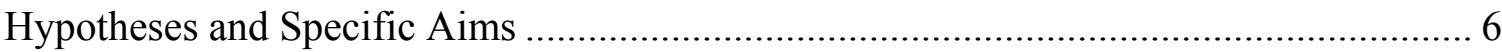

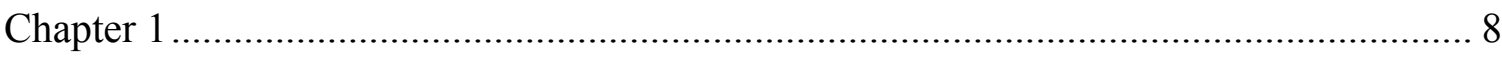

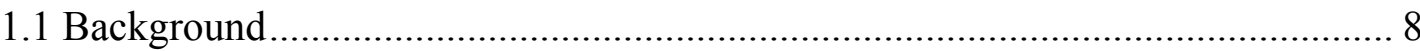

1.1.2 Two-Dimensional cultures vs. Three-Dimensional models........................... 10

1.1.3 Multicellular tumor spheroids:................................................................... 12

1.1.4 Tumor spheroids as drug screening tools.................................................... 12

1.1.5 Basic culture techniques of spheroids........................................................ 13

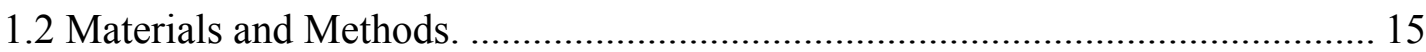

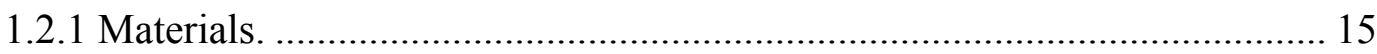

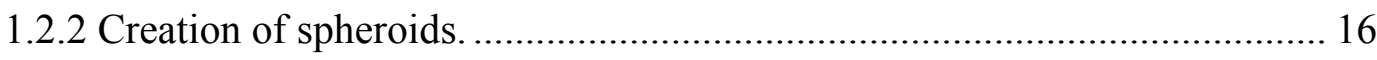

1.2.3 Characterization of spheroids ................................................................ 17

1.2.4 Cytotoxicity assessment under Doxorubicin chemotherapy and adjuvant

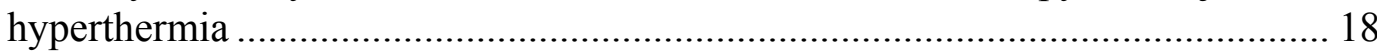

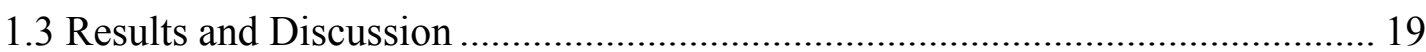

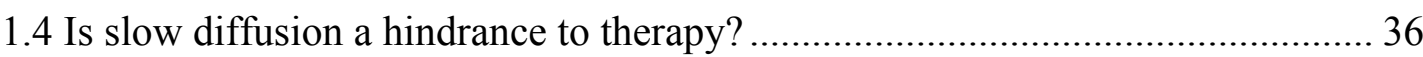

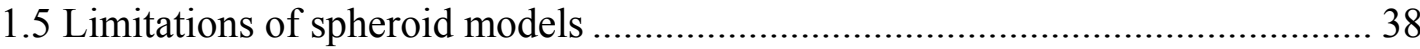

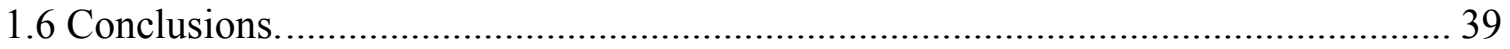

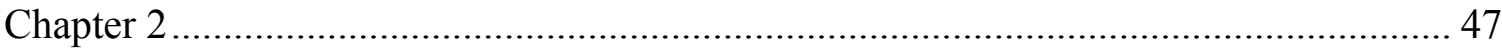

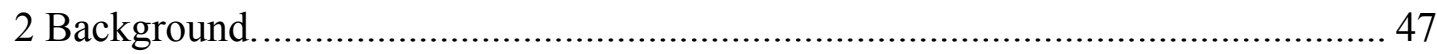

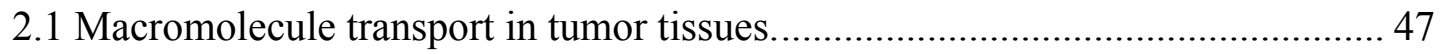

2.2 Mathematical description of transport in tumor spheroids................................... 49

2.3 Mathematical models of cell uptake kinetics......................................................... 53

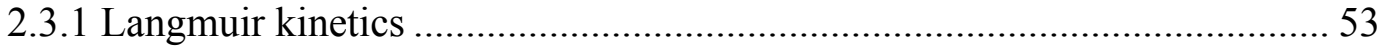

2.3.2 Determination of cell uptake constants using Langmuir kinetics................ 54

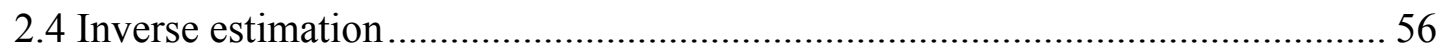

2.5 Silica nanoparticles as model nanoparticles for cell uptake. ................................. 60 


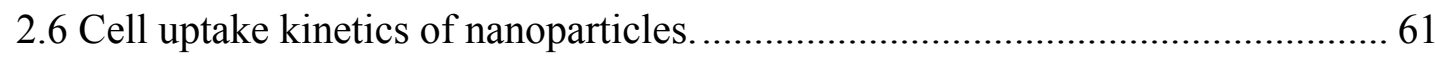

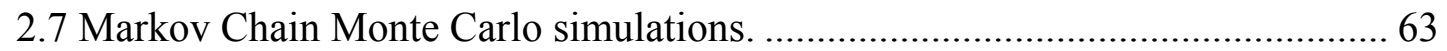

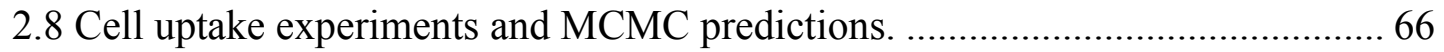

2.9 Simulating nanoparticle transport in spheroids under hyperthermia................. 71

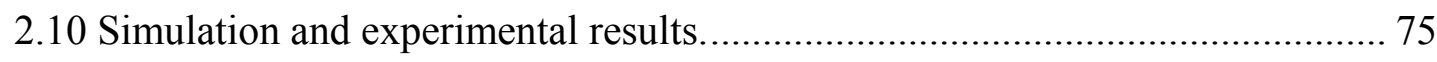

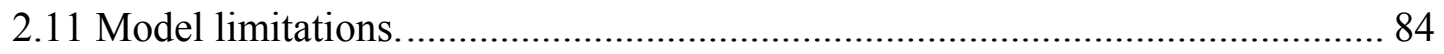

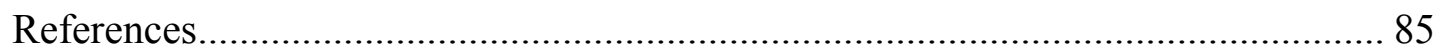

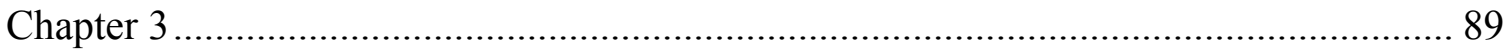

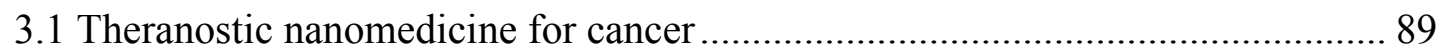

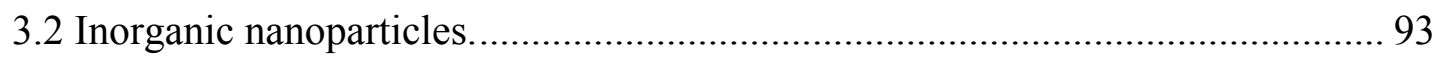

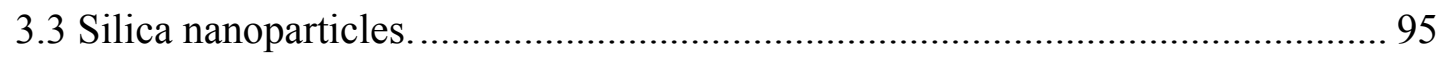

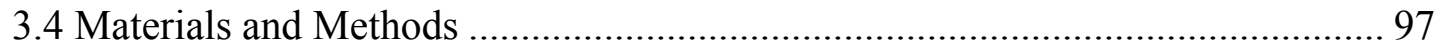

3.4.1 DOX IR820 Loaded Ormosil Synthesis ................................................... 97

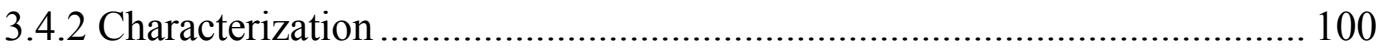

3.4.3 Absorbance, Fluorescence and Release kinetics.................................... 100

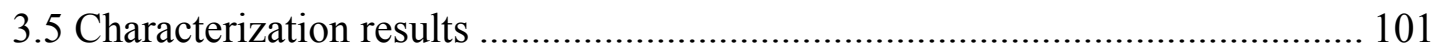

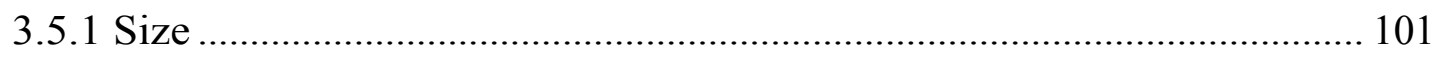

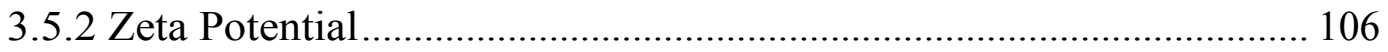

3.5.3 FTIR, Absorbance results ............................................................... 108

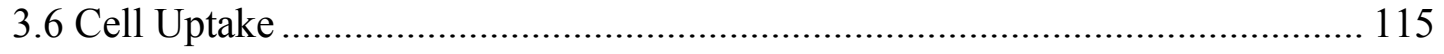

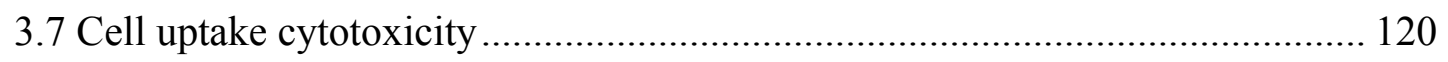

3.7.1 Cytotoxicity of Different Ormosil Formulations in Skov-3 cells ............. 121

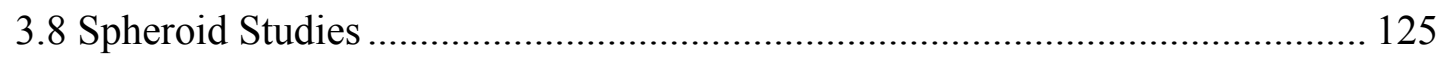

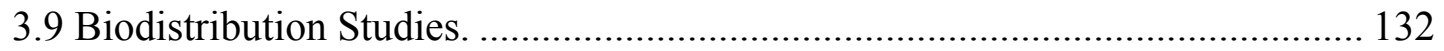

3.10 Nanoparticle Design Criteria for Cancer Theranostics................................ 139

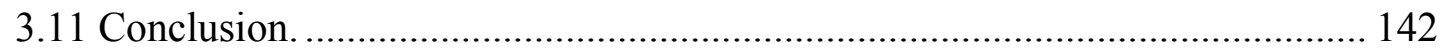

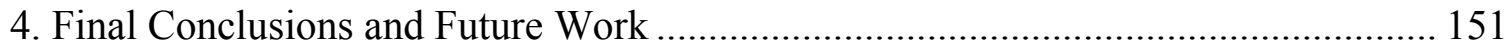

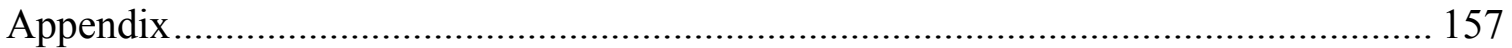

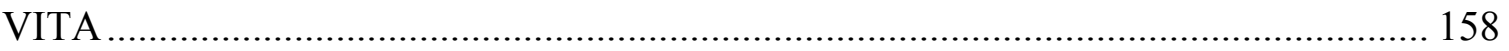




\section{LIST OF FIGURES}

FIGURE

PAGE

Figure 1.1 : Stages of Spheroid Formation a) initial clustering, b) reorganization of clusters, c) aggregation, d) compaction.

Figure 1.2 : Brightfield images of spheroids. Images were taken using $4 \mathrm{X}$ objective,

Skov3 (left) and Dx-5 (right). Scale bar at the bottom left of images is $200 \mu \mathrm{m}$. 23

Figure 1.3: SEM Images of Skov-3 Spheroids show compact organization, 350X magnification (left) and 1,900X magnification showing the surface (right)....

Figure 1.4: SEM Images of MES-SA/Dx-5 Spheroids show distinct cell bodies. 1000X magnification (left) showing disperse cellular organization and 10,000X magnification (right) showing the individual arrangement of cells. Visible perforations on the cell bodies may have been due to tissue processing.

Figure 1.5: Histological analysis of Skov-3 (left) and MES-SA/Dx-5 (right) spheroids show the stratified organization of the spheroids. Spheroids show a dense cuticular layer (outside) followed by a lighter core containing necrotic cells (lighter appearance and shrunk cell nuclei). The empty spaces in the images are due to the loss in sample during histological processing.

Figure 1.6: DOX + HYP therapy causes cell death at the spheroid periphery. (Top) Skov-3 control spheroids without any treatment, the growth of the spheroid over time is evident, (Bottom) Skov-3 Spheroids under DOX treatment, images show a shrinking spheroid with progress of time. Scale bar represents $100 \mu \mathrm{m}$.

Figure 1.7: MES-SA/Dx-5 spheroids under combined DOX + HYP treatment over 6 days. Scale bar represents $200 \mu \mathrm{m}$.

Figure 1.8: Growth Curves of Skov-3 (left) and MES-SA/Dx5 spheroids show reduction in spheroid volume. Data is represented as mean \pm stdev of $n=4, *$ indicates significant difference $(\mathrm{p}<0.05)$ between control and treatment. The growth ratios were calculated by dividing the corresponding day volume by the initial volume of the spheroid before treatment.

Figure 1.9:Alamar blue assay results showing the presence of viable cells at the end of treatment i.e., day 6. $\mathrm{P}<0.05$ indicates significant difference between the control and different treatments. No significance was found for mean cell viability $(n=6)$ between DOX and DOX + HYP treatment.

Figure 2.1: Trace plots showing chain mixing of parameters. $\mathrm{k}_{\mathrm{a}}, \mathrm{k}_{\mathrm{d}}$ and $\mathrm{ki}$, show good mixing while $\mathrm{k}_{\mathrm{rec}}$ shows marginal mixing. 
Figure 2.2: Parameter prediction is not dependent on the chosen initial guess. Results show no dependence on initial guess.

Figure 2.3: MCMC Predictions align closely with experimental measurements for all cases. Image shows predictions of cell uptake overlaid with experimental data.

Figure 2.4: MCMC Predictions align closely with experimental measurements for all cases. Image shows predictions of cell uptake overlaid with experimental data.

Figure 2.5: Schematic illustrating the Multiphysics strategy used in COMSOL. ............ 73

Figure 2.6: Plots showing reduction in cell viability under hyperthermia (left) and changes in effective diffusion coefficient as a result of particle aggregation and change in porosity (right).

Figure 2.7 : FITC fluorescence is concentrated around the periphery of the spheroid. Confocal sections of Skov-3 spheroids incubated with cPEGSi at $37^{\circ} \mathrm{C}$ with DAPI and FITC channels merged.

Figure 2.8: FITC fluorescence is more diffuse and is not found around the periphery. Confocal sections of Skov-3 spheroids incubated with cPEGSi at $43^{\circ} \mathrm{C}$ with DAPI and FITC channels merged.

Figure 2.9: Overlay of model predictions with experimental data. The experimental data plotted as two curves denote the higher and lower limits of standard error $(\mathrm{n}=3)$.

Figure 2.10: COMSOL simulations comparing of transport of $50 \mathrm{~nm}$ gold nanoparticles with high binding affinity to cells and cPEGSi. The uptake parameters for gold nanoparticles were from studies by Jin et al. 2009. The inset shows the particle distribution (zoomed) inside the spheroid.

Figure 3.1: Schematic of nanoparticle formation in microemulsion and different modes of DOX loading.

Figure 3.2: Intensity size distribution of Ormosil nanoparticles in dulbeccos phosphate buffered saline show that the mean diameter of the particles is at $58 \mathrm{~nm}$ 's.

Figure 3.3: Scanning electron microscopy of CDSIR 820 particles show monodisperse spherical particles

Figure 3.4: FITR-ATR spectra shows core shell structure of CDSIR820. Image shows FTIR-ATR spectra of Blank ORM, CDSIR820, Free Dox and Free IR820. A clear overlap is seen for Blank ORM (no drug or dye) with CDSIR820 indicating the presence of an additional silica shell. 
Figure 3.5: Fluorescence studies show leakage of DOX from FDSIR820 in McCoy's $5 \mathrm{~A}$ media. Fluorescence spectra of different $\mathrm{Si}$ formulations $(3.2 \mu \mathrm{M}$ equivalent DOX concentration) were acquired in DPBS (Left) and McCoy's 5A cell culture media (Right).

Figure 3.6: Absorbance curves of Free DOX and IR820 mixture in DI water and PEGCDSIR820 in DI water.

Figure 3.7: Exposure of PEGCDSIR820 to NIR laser resulted in temperature raise. Temperature raise profile of PEGCDSIR820 for successive laser exposures 1 hour apart.

Figure 3.8: Drug (DOX) and Dye (IR820) are retained by ORM formulations in dulbeccos phosphate buffered saline at $37^{\circ} \mathrm{C}$ (left). NIR Exposure of PEGCDSIR820 lead to release of DOX. Particles were periodically exposed to laser every hour over a period of 3 hours (right). The error bars represent standard error of mean $(n=3) \ldots \ldots .115$

Figure 3.9: Confocal images show differences in intracellular localization of DOX between FDSIR820 and CDSIR820. Fluorescence microscope images of Skov-3 cells incubated with different Ormosil formulations in different fluorescence channels. Scale bar shown in left bottom corner is $40 \mu \mathrm{m}$. White arrows indicate DOX fluorescence from the nuclear region.

Figure 3.10 : DOX released from PEGCDSIR820 on NIR exposure trans locates to the cell nucleus. Confocal Images of DOX and PEGCDSIR820 distribution in Skov-3 cells. Top panel shows DOX fluorescence from cell nuclei. Middle panel shows fluorescent DOX puncta with minimal fluorescence from the nuclei. Bottom panel shows Skov-3 cells with PEGCDSIR820 particles after laser exposure. DOX fluorescence is evident from cell nucleus. Laser exposed cells show fragmented and shrunk nucleus indicating cell necrosis.

Figure 3.11: Dose dependent cytotoxicity of different nanoparticle formulations under presence or absence of laser exposure. Comparison of FDSIR820 and CDSIR820 (left). Effect of Pegylation and laser exposure on cytotoxicity of PEGCDSIR820. The error bars represent standard error of the mean $(n=3)$.

Figure 3.12: Silica nanoparticles affect the cell mitochondria. Cells were stained with Mitotracker Red as per manufacturer's instructions. Green puncta indicate nanoparticles. Cells incubated with aminated silica particles show fragmented mitochondrial cristae (left). Carboxylated silica nanoparticles did not induce mitochondrial fragmentation (right). Images were taken with $100 \mathrm{X}$ objective using the DeltaVision ${ }^{\circledR}$ deconvolution Microscope.

Figure 3.13 : Confocal Images of $90 \mu \mathrm{m}$ deep sections of Skov-3 spheroids from 2hour study in which spheroids were incubated with PEGCDSIR820 for 2 hours and then exposed to laser 3 times. Images were collected after 8 hours. Top panel is the 
image of PEGCDISR820 particles with no laser exposure. DOX fluorescence is concentrated around the periphery. Bottom panel shows image of PEGCDSIR820 particles with laser exposure. DOX and DAPI fluorescence is more diffuse in the spheroid following laser exposure compared to the no-exposure group. Scale bar is $100 \mu \mathrm{m}$

Figure 3.14: Exposure to NIR hyperthermia reduced the cell uptake of PEGFITCORM nanoparticles. Uptake of PEGylated Fluorescein Isothiocyanate loaded Ormosil particles (PEGFITCORM) by Skov-3 cells after 24 hours under different temperature conditions (left), $(*)$ represents significant difference between uptake at $37^{\circ} \mathrm{C}$ (PEGFITCORM+37) and NIR exposure (PEGFITCORM+NIR).

Figure 3.15 : Evidence of autophagic uptake (different uptake mechanism compared to endocytosis) of silica nanoparticles under hypoxia. Cells were subject to $1 \% \mathrm{PO}_{2}$ (Hypoxia) for $18 \mathrm{hrs}$. followed by incubation with Si nanoparticles for 24 hours. Autophagy was imaged by staining cells with Monodansylcadaverine.

Figure 3.16: Exposure to NIR hyperthermia of PEGCDSIR820 lead to spheroid toxicity.Top left image shows Skov-3 spheroids incubated with PEGCDSIR820, the compact structure is apparent with some loose cells at the periphery. Bottom left shows a less compact spheroid as a result of cell dissociation and death due to PEGCDSIR820 + Laser treatment (scale bar is $100 \mu \mathrm{m}$ ). Trypan blue viability results for spheroids from the 24-hour study. Spheroids were incubated with PEGCDSIR 820 for 24 hours and then exposed to laser 3 times. Trypan blue assay was done 24 hours after laser exposure. Data is represented as Mean \pm S.E $\mathrm{n}=8,\left({ }^{*}\right)$ indicates significance for $\mathrm{p}<0.05$ and $(* *)$ indicates significance at $\mathrm{p}<0.001$ level.

Figure 3.17: High NIR fluorescence is observed from the internal RES organs of mice. Fluorescence images of different organs at different time points obtained from Li-Cor imaging system.

Figure 3.18 : Plasma pharmacokinetics show increased circulation time of IR820 administered using PEGCDSIR820 particles.Plasma clearance curves of IR820 vs Time after IV injection of PEGCDSIR820 particles. Y-Axis is shown in log units. Data is represented as Mean \pm S.E of $n=3$.

Figure 3.19: Simulation of the effect of plasma clearance of nanoparticles on tumor accumulation. 
Introduction.

Chemotherapy of cancer uses cytotoxic agents to target rapidly multiplying cancer cells. In recent years, several classes of chemotherapeutic drugs, viz, campothecins, platinates and anthracyclines have been researched for cancer therapy. However, most of these drugs suffer from poor bioavailability, poor pharmacokinetics and severe systemic toxicity due to the exposure of drug to healthy organs that leads to adverse side effects (Venditto and Szoka, 2013; Wilhelm et al., 2016). Current research has focused on developing analogs of the aforementioned drugs with improved pharmacokinetics, solubility and reduced side effects. This is achieved either by structural modifications to the drug molecule or by encapsulating the drug in a special class of delivery vehicles known as nanocarriers/nanoparticles. Recent advances in material science have provided a vast array of materials such as polymers, lipids, metals and organo-metals with unique advantages for the preparation of nanoparticles for delivery of therapeutic agents. For example, liposomes are formulated from lipids are lamellar vesicles that consist of a hydrophobic tail and a hydrophilic head. The tail can retain hydrophobic drugs and the head promotes the solubility of drug carrier in aqueous media which makes it suitable for in-vivo injection. Similarly, amphiphilic polymers such as Poly-Lactic co-Glycolic Acid (PLGA) are used to simultaneously encapsulate hydrophilic and hydrophobic agents (Manchanda et al., 2010). From a drug delivery perspective, nanoparticles offer several advantages; 1) passive accumulation at the tumor $\operatorname{site}^{1}$ via the enhanced permeation and

\footnotetext{
1 Tumor site comprises of the tumor tissue, surrounding vasculature and the interstitial space.
} 
retention effect (EPR). EPR arises as a result of discontinuous fenestrations of poorly developed tumor vasculature that leads to preferential extravasation of the nanocarrier, 2) active targeting of the tumor by tagging the nanocarrier with ligands that are specific to cancer cell receptors, and 3) modifying nanoparticle surface with "stealth" materials such as PolyEthylene Glycol (PEG) to reduce clearance of nanoparticles by the reticulo endothelial system (RES) (Ferrari, 2005). Naturally, these advantages have led to extensive research of nano-formulations for cancer drug delivery.

There have been close to three million publications related to cancer nanomedicine since 1945 . However, only $3 \%$ of total research has been translated to clinical trials and an even a smaller fraction has progressed to actual therapy in the clinic (Venditto and Szoka, 2013). Antibody based drug conjugates and liposomal formulations of drugs have had greater success in progressing to clinical trials compared to polymer based nanoparticles. Some notable examples among antibody therapies are Rituxan ${ }^{\circledR}(\mathrm{CD} 20$ based, Genentech) for chronic lymphocytic leukemia and Herceptin ${ }^{\circledR}$ (HER-2 receptor targeted, Genentech/Roche) for HER-2 positive breast and gastric cancer. Examples of liposomal based nanomedicines include DOXIL $®$ (Johnson \& Johnson) for platinum therapy resistant ovarian cancer, Daunoxome ${ }^{\circledR}$ (Gilead) and Myocet ${ }^{\circledR}$ (Enzon) for metastatic breast cancer (liposomal formulations of Doxorubicin). Despite the above mentioned advantages of nanomedicine, one of the main reasons for such low translation from research to the clinic is that nanomedicines have failed to increase the therapeutic index of encapsulated drug compared to its free form. Nanomedicines that show great success in in-vitro studies during the innovation phase often fail to show improvement over 
the un-encapsulated (free) form in the translation (in-vivo) phase. It has been estimated that only $0.7 \%$ (median value from various studies) of the drug injected as nanoparticles reaches the tumor (Wilhelm et al., 2016). Another challenge at the tumor site that further limits the efficacy of nanomedicine is the limited penetration of nanoparticles into the tumors. This aspect (limited penetration) of delivery of nanoparticles has been largely ignored (Curtis and Frieboes, 2016; Minchinton and Tannock, 2006). Along-with increasing the availability of nanoparticles at the tumor site, the innovation strategy for nanomedicine should also consider improving the distribution of nanoparticles in tumors in order to increase the efficacy of therapy. One way to achieve this goal is by using rigorous models (experimental and mathematical) to screen the nano-formulations at the in-vitro stage. These models should simulate or recreate the structural and functional characteristics of a tumor of interest as closely as possible. This will ensure efficient screening of nanomedicines and their properties in relation to the properties of the tumor in order to predict a set of optimal nanoparticle properties that may be effective for deep delivery. Additionally, mathematical models can simulate several combinations of the properties of a nanoparticle in order to predict optimal properties of the nanoparticle that are suitable for delivery to the tumor in question. This will reduce the number of experiments that need to be performed (as nanoparticles with low success can be eliminated prior to experimentation) which will also reduce the cost associated with developing nanomedicines for cancer therapy

Another strategy to enhance the effect of nanoparticle based chemotherapy at the tumor site is by using a combination of therapies, i.e., an adjuvant therapy. By properly 
tailoring or choosing the properties of the material from which the nanoparticle is formulated, more than one agent (imaging, drugs etc.) can be simultaneously encapsulated within a nanoparticle system. Moreover, the nanoparticle can be functionalized with targeting and stealth properties for specific targeting of the tumor and evasion from reticuloendothelial clearance. Various functions, i.e., imaging, targeting, adjuvant treatments etc. can thus be achieved with such a multifunctional system.

In this study three dimensional tumor spheroids were used as experimental models to study nanoparticle distribution in avascular tumors. The effect of the combination of Doxorubicin with adjuvant hyperthermia $\left(43^{\circ} \mathrm{C}\right)$ was studied in tumor spheroids. Hyperthermia was further investigated as a modality to improve nanoparticle distribution in the tumor. A novel inverse estimation method was developed to predict cell uptake kinetics of nanoparticles under hyperthermia. This was combined with a mathematical model of advection- reaction transport to predict the transport of nanoparticles in spheroids under hyperthermia. A novel multifunctional nanoparticle system capable of delivering chemotherapy, rapid rise adjuvant hyperthermia and near infrared imaging was developed and tested for efficacy in tumor spheroids. The conclusions from this study will be valuable for planning hyperthermia based adjuvant therapies with nanoparticles as well as for using hyperthermia to enhance nanoparticle transport in avascular tumors.

\section{References}

1. Curtis, L. T.; Frieboes, H. B. In The Tumor Microenvironment as a Barrier to Cancer Nanotherapy; Systems Biology of Tumor Microenvironment; Springer: 2016; pp 165-190. 
2. Ferrari, M. Cancer nanotechnology: opportunities and challenges. Nature Reviews Cancer 2005, 5, 161-171.

3. Manchanda, R.; Fernandez-Fernandez, A.; Nagesetti, A.; McGoron, A. J. Preparation and characterization of a polymeric (PLGA) nanoparticulate drug delivery system with simultaneous incorporation of chemotherapeutic and thermo-optical agents. Colloids and Surfaces B: Biointerfaces 2010, 75, 260-267.

4. Minchinton, A. I.; Tannock, I. F. Drug penetration in solid tumours. Nature Reviews Cancer 2006, 6, 583-592.

5. Venditto, V. J.; Szoka, F. C. Cancer nanomedicines: so many papers and so few drugs! Adv. Drug Deliv. Rev. 2013, 65, 80-88.

6. Wilhelm, S.; Tavares, A. J.; Dai, Q.; Ohta, S.; Audet, J.; Dvorak, H. F.; Chan, W. C. Analysis of nanoparticle delivery to tumours. Nature Reviews Materials 2016, l, 16014. 


\section{Hypotheses and Specific Aims}

Objective Statement:

The first objective of this study is to determine the effect of combination of hyperthermia on doxorubicin therapy in multicellular tumor spheroids. The second objective is to study the effect of hyperthermia on nanoparticle transport in multicellular tumor spheroids.

Objective A:

Create multicellular tumor spheroids to test the combination of Doxorubicin and adjuvant hyperthermia $\left(43^{\circ} \mathrm{C}\right)$ therapy.

Hypothesis for Objective A:

Multicellular tumor spheroids are more resistant to Doxorubicin and to the combination of Doxorubicin and adjuvant hyperthermia than are monolayer cultures.

Specific Aims for Objective A:

1) Create and characterize a 3-dimensional tumor model (spheroids) with ovarian (Skov3) and uterine (MES-SA/DX5 and MES-SA) cancer cell lines.

2) Study the effect of Doxorubicin chemotherapy and adjuvant hyperthermia on Skov3, MES-SA and MES-SA/DX5 cell spheroids.

Objective B:

Estimate the parameters for cellular uptake kinetics of nanoparticles and formulate a mathematical model for describing nanoparticle transport in spheroids under hyperthermia. 
Hypothesis of Objective B

Mild-hyperthermia will increase the penetration of nanoparticles into tumor spheroids by increasing the porosity (denoted $\varepsilon$ ) of the spheroid tissue.

Specific Aims for Objective B:

1) Develop a theoretical/mathematical model to study changes in drug diffusion in a tumor spheroid model under mild-hyperthermia.

2) Validate the theoretical model by performing drug penetration studies in spheroids under mild-hyperthermia.

Objective C:

Create a multifunctional theranostic nanoparticle system capable of imaging, chemotherapy and adjuvant hyperthermia.

Specific Aims for objective C

1) Synthesize a multifunctional theranostic platform by encapsulating DOX and IR820

2) Evaluate the system characteristics i.e., size, release kinetics of IR820, DOX and efficacy i.e., cytotoxicity in in-vitro cell culture and spheroids.

3) Study cell uptake and DOX penetration into spheroids under NIR hyperthermia.

4) Obtain Bio-distribution profile in mice. 


\section{Chapter 1}

\subsection{Background}

Anthracycline drugs such as Doxorubicin (DOX) are DNA intercalating agents that inhibit topoisomerase II and prevent DNA stabilization. The main disadvantage of DOX is the systemic side effect, notably irreversible cardiotoxicity caused by the lack of tissue and organ specificity. Moreover, cancer cells may develop multidrug resistance through expression of $\mathrm{P}$-glycoprotein (P-gp). DOX is a $\mathrm{P}$-gp substrate which leads to its active exclusion from MDR cells via the P-gp pump. The use of P-gp inhibitors such as Verapamil hydrochloride in combination with DOX has been shown to enhance DOX retention in P-gp positive cells. However, these agents are by themselves also toxic. Another strategy to enhance the efficacy of cancer therapy is to use a combination of different modalities i.e., chemotherapy and radiotherapy, surgery and chemotherapy, etc. (Fotopoulou et al., 2010; Macchiarini et al., 1991; Olivo et al., 2010; Wolf et al., 1991). Our lab has made valuable contributions to the research of combining chemotherapy with adjuvant hyperthermia at $43^{\circ} \mathrm{C}$ (Tang, 2010; Tang and McGoron, 2009).

Hyperthermia (Hyp) (also called thermal therapy, thermotherapy) is the application of heat $\left(41^{\circ} \mathrm{C} \sim 45^{\circ} \mathrm{C}\right)$ to selectively kill cancer cells. Healthy cells are less susceptible to heat than are cancer cells (van der Zee, 2002). At high temperature, cell proteins coagulate or get damaged leading to activation of cell killing pathways. The mode of cell killing, i.e., apoptosis or necrosis, is determined by the rate of heating. We have found that long duration slow rate hyperthermia at $43^{\circ} \mathrm{C}$ induced mild cell apoptosis while, short duration fast rate hyperthermia causes necrosis in uterine sarcoma cells (Tang, 2010). These benefits can be combined with DOX chemotherapy to achieve a synergistic, or at least a more than 
additive effect of independent treatments. Hyperthermia can be administered to the whole body or locally through microwaves, radio waves and via the sequestration of photo thermal agents that generate heat on laser exposure at the tumor site. Our recent work has concentrated on evaluating the mechanisms of cell killing in chemotherapy and adjuvant hyperthermia (Tang, 2010), the evaluation of the near infrared (NIR) imaging dyes Indocyanine Green and IR820 for laser guided local hyperthermia (Fernandez-Fernandez et al., 2012). Multifunctional carrier systems (nanoparticles) that enhance the stability of DOX, ICG and IR820 against degradation were also formulated from different polymer materials and were evaluated for cell killing potential in Ovarian (Skov-3) and Uterine (MES-SA/DX5) cancer cells. Studies in monolayer in-vitro cell cultures have shown great promise for chemotherapy and adjuvant hyperthermia, especially when delivered through encapsulation of DOX, IR820 or ICG in nanoparticles. However, further studies are needed in order to assess the efficacy of the aforementioned approach for in-vivo applications due to the complex nature of the tumor microenvironment (stroma).

Tumor stroma is a critical determinant of the response to chemotherapy and adjuvant hyperthermia due to the presence of different cell types (fibroblasts, macrophages), gradients of oxygen, low $\mathrm{pH}$ and abnormally developed vasculature (Hirschhaeuser et al., 2010; Sutherland, 1988). The extracellular matrix (ECM) surrounding the tumor stroma is composed of glycosaminoglycans, laminin, collagen, fibronectin and hyaluronic acid (HA). The ECM and opposing interstitial fluid pressure are a barrier to the transport of macromolecules and solutes. Oxygen and nutrient levels have been found to drop rapidly in tumor tissues with an increase in distance from the nearest blood supply. Reduction of oxygen leads to regions of hypoxia. Reduced nutrient 
availability (glucose) and lack of drainage of waste products of cell metabolism (lactic acid) result in regions of low $\mathrm{pH}$. Cells that are exposed to these conditions (hypoxia, low pH) for long durations respond differently to therapy (Jain, 1999; Tredan et al., 2007a). Hence, when planning chemotherapy with adjuvant hyperthermia the functional complexity of tumor stroma should be taken into account.

\subsubsection{Two-Dimensional cultures vs. Three-Dimensional models}

Two-dimensional cultures (2-D) do not possess the structural complexity of an invivo tumor required to simulate macromolecule transport and the associated response of cells in relation to the conditions of the tumor microenvironment. Hypoxia, low $\mathrm{pH}$ and low glucose metabolism can be created in 2-dimensional cultures (2-D cultures). However, it is not possible to generate heterogeneous populations of cells that arise as a result of these conditions in a tumor tissue. Also, some cells, when cultured in 2-D cultures lose their functional phenotype (Abbott, 2003). The shortcomings of a 2-D model can be addressed by three-dimensional cultures (3-D). Cells grown in 3-D cultures have been shown to regain their functional phenotype. Bokhari et al. have shown that hepatocytes grown in 3-D scaffolds are able to regain their functionality (albumin production) that was lost when the cells were grown in 2-D (Bokhari et al., 2007). Similarly, breast epithelial cells formed structures that resembled in-vivo acini when cultured in 3-D (Debnath et al., 2003). Moreover, the gene profile of 3-D cultures resembles more closely the clinical expression profiles of in-vivo tumors when compared to 2-D cell cultures (Hirschhaeuser et al., 2010). 
Acellular Models: Gels such as agarose and hydrogels can be formulated to possess the basic physical properties (density, porosity) of ECM. They can be considered the simplest models of "tissue" and are used to study the effects of changes in the physical parameters of macromolecules, viz size, shape and surface charge, on macromolecule transport and distribution. Acellular models have also been used to study the effect of external stimuli (light, heat and chemical) on the change in gel structure and its corresponding influence on molecule transport (Sykes et al., 2016; Wong et al., 2011b). However, since these models do not have cells they cannot be used for studying the functional effects of macromolecules on cells, such as the response to therapy and corresponding effects of the cells on the ECM (Goodman et al., 2008).

In-vivo/Ex-vivo models: Tumors grown in an animal host are ideal for studying drug, antibody and nanoparticle transport. They are beneficial for real time monitoring of macromolecule transport using techniques such as intravital microscopy and confocal microscopy (for excised tumors). Furthermore, animal models are also used to study the pharmacokinetics and bio-distribution of the administered macromolecules. A very large data set related to drug bio-distribution, clearance/residence time of drugs in pharmacokinetic compartments and drug efficacy or side effects can be collected from a single experiment (Goodman et al., 2008). Ex-vivo models correspond to tumor tissues that have been harvested directly from human subjects or animals. Ex-vivo models have been used to study the distribution of macromolecules in tumor tissues. However, studies in invivo/ex-vivo models are expensive, time consuming, and require significant expertise to 
control the experimental variables, which might result in the limited reproducibility of results.

\subsubsection{Multicellular tumor spheroids:}

Tumor spheroids are models of small tumor tissues that are grown in in-vitro conditions. Tumor spheroids are formed when cells aggregate in culture due to cellular characteristics (microvilli, membrane interdigitations) or through secretion of ECM. The formation can be further augmented by supplementing the culture with ECM components such as collagen, fibronectin or an ECM alternative (Matrigel ${ }^{\circledR}$ ). Spheroids mimic the structural and functional features of a tumor microenvironment, which makes them attractive candidates to study macromolecule transport and distribution within the tumor. Gradients of nutrients, $\mathrm{pH}$ and oxygen have been found in tumor spheroids (Acker et al., 1987; Sutherland, 1988). Structural features such as a dense ECM around the aggregated cells and in some cases, functional cell-cell contacts such as E-cadherin junctions and gap/tight junctions (desmosomes) have also been found in tumor spheroids (Cottin et al., 2010). Therefore, tumor spheroids are suitable models of in-vivo avascular tumors such as micro metastases and early stage tumors.

\subsubsection{Tumor spheroids as drug screening tools.}

The use of cancer spheroids as a research tool to study the cellular-response to therapeutic interventions can be found as early as the late 1970's (Yuhas et al., 1977). Tumor spheroids have been used to study the response of cancer cells to radiotherapy, photodynamic treatment, hyperthermia, chemotherapy including antibody and gene based 
therapies (Hirschhaeuser et al., 2010). They have also been routinely used to quantify drug distributions using techniques such as fluorescence microscopy and autoradiography. Multicellular tumor spheroids have also been used to study the penetration of antibodies (Weinstein et al., 1987), nanoparticles (Goodman et al., 2007) and SiRNA (Wong et al., 2011a) in avascular tumors.

\subsubsection{Basic culture techniques of spheroids}

Hanging Drop Cultures: Cell suspensions of 15-20 $\mu 1$ are plated in a dish and the dish is inverted. The drops are held in place due to surface tension. Cells aggregate with each other due to the formation of intracellular contacts under the action of gravity and form spheroids. Cell spheroids of different cell types have been reported using this method (Kelm et al., 2003; Timmins and Nielsen, 2007). The hanging drop method is consistent in producing spheroids of the same size since the size is dependent only upon the initial seeding density of cells. However, the major disadvantages of this method include; 1) the small volume of cell-suspension that is susceptible to evaporation which leads to dry-out in extreme cases or a change in nutrient concentration available to the cells (hence the hanging drop cultures usually require moisture chambers), 2) the small volume of cell suspension places a limit on the size of spheroids that can be cultured, and 3) cultures must not be disturbed during spheroid growth as it may interrupt cell-cell organization and arrest spheroid growth. Since the cultures cannot be disturbed, the addition of supplements for spheroid growth (Matrigel ${ }^{\circledR}$, collagen and fibronectin) is difficult to achieve in a hanging drop culture. 
Spinner Flask Cultures: These cultures are done in large vessels (150-300 ml) of media that are continuously rotated at a fixed rate. Constant rotation inhibits adhesion of cells to the surface of the vessel and enhances interactions between the cells. This leads to the formation of spheroids (Lin and Chang, 2008). Spinner flask cultures can lead to the formation of a large number of spheroids. However, spinner flask cultures demand a lot of materials in terms of cell culture media and supplements. Also, due to the large volume of media, carbon dioxide and oxygen needs to be perfused through the culture vessel at regular intervals of time to maintain the $\mathrm{pH}$ and oxygen balance. Although spinner flasks can produce a large number of spheroids, there are variations in shape and size of the spheroids with this method. Hence, in experiments where spheroid size is important, additional techniques such as sieving through nylon meshes to select similar size spheroids are necessary (Lin and Chang, 2008).

Liquid Overlay Culture/Use of cell-adhesion inhibition surfaces: Liquid overlay cultures are performed by coating the tissue culture plate with agarose (Carlsson and Yuhas, 1984), PolyHEMA (Ivascu and Kubbies, 2006) or poly- $N$-p-vinylbenzyl-Dlactonamide (Hou et al., 2001). These materials prevent the adhesion of cells to the culture plate and promote cell-to-cell interactions resulting in the formation of spheroids. Liquid overlay cultures are usually performed in 96 or 48 well formats. The advantages of this system include; 1) A large number of spheroids can be cultured in a multi well format (96, 48 wells, etc.) facilitating high throughput drug screening. Although, for doing high throughput culture specialized equipment such as an automated liquid dispensing system and micro patterned substrates are needed (Kikuchi et al., 2009; Xu et al., 2011); 2) 
Spheroids can be cultured with minor modifications to the culture plate and the size of spheroids can be easily controlled by selecting the initial seeding density of the cells. Despite these advantages liquid overlay cultures are very sensitive to the experimental protocol, i.e., type of agarose used and coating of agarose. Minor modifications can lead to some, or even a complete inhibition of spheroid formation (Friedrich et al., 2009).

Other methods: Other techniques described in the literature to produce tumor spheroids include the use of rotary bioreactors (Ingram et al., 1997), culture in 3-D scaffolds (Fischbach et al., 2007) and linker-engineered spheroids where the primary aggregation between the cells is maintained through a cell-linker, where the linker gradually degrades as the cell spheroid grows (Ong et al., 2010).

1.2 Materials and Methods.

\subsubsection{Materials.}

Ovarian cancer cells (Skov-3), uterine sarcoma cells (MES-SA), drug resistant uterine sarcoma cells (MES-SA/Dx5), McCoy's 5A media, Fetal Bovine Serum (FBS) and penicillin/streptomycin were obtained from American Type Culture Collection (ATCC). Culture media was prepared by supplementing McCoy's 5A with $10 \%$ (v/v) FBS and $1 \%$ (v/v) penicillin/streptomycin. Matrigel ${ }^{\circ}$, an extracellular membrane extract derived from a mouse hybridosarcoma, was purchased from BD Biosciences. Doxorubicin Hydrochloride (DOX-HCl) was obtained from Watershed Technologies. Histology and scanning electron microscopy supplies, such as low melting paraffin, Permount ${ }^{\circledR}$ (mounting medium), hematoxylin, eosin and osmium tetroxide were obtained from Electron Microscopy Sciences. Glutaraldehyde was acquired from Sigma Aldrich. 


\subsubsection{Creation of spheroids.}

Spheroids were cultured using the liquid overlay method (Carlsson and Yuhas, 1984; Yuhas et al., 1977). Ninety-six well plates were prepared for spheroid culture by coating with $2.5 \%(\mathrm{w} / \mathrm{v})$ agarose gel. Briefly, $0.313 \mathrm{~g}$ agarose was dissolved in $12.5 \mathrm{ml}$ PBS and stirred on a hot plate at medium speed ( $\sim 800$ r.p.m) until the solution became transparent. Then, the solution was sterilized by autoclaving. Each well was coated with $50 \mu 1$ agarose gel and the gel was allowed to solidify at room temperature. Agarose coating was done freshly before every culture.

Skov-3, MES-SA/Dx5 and MES-SA cells were maintained in T-25 $\left(25 \mathrm{~mm}^{2}\right.$ growth area) cell culture flasks under standard conditions, i.e., $5 \% \mathrm{CO}_{2}, 37^{\circ} \mathrm{C}$ and $95 \%$ humidity. They were fed with McCoy's 5 A media as recommended by ATCC. For spheroid preparation, cells were washed with DPBS and detached from the surface using trypsin EDTA (EDTA concentration: $0.025 \%(\mathrm{v} / \mathrm{v})$ ). Trypsinized cells were centrifuged at 1500 r.p.m (300 g) for 4 minutes after which the supernatant was discarded and the cell pellet was re-suspended in fresh McCoy's 5A media. Cells were counted using a Neubaeur hemocytometer and diluted with McCoy's 5A media to 25,000 cells per milliliter. Two hundred microliters of diluted cell-suspension was placed in each well (5000 cells/well). The plates were shaken briefly and incubated under standard conditions (Day $=0)$. On Day $3,5 \mu 12.5 \%(\mathrm{v} / \mathrm{v})$ Matrigel solution was prepared in ice-cold media and transferred to every well. The plates were further incubated. On Day 5, the spheroids were ready for performing further tests. All spheroids were used on day 5 unless otherwise specified. 


\subsubsection{Characterization of spheroids}

Histology: Spheroids were embedded in an agarose plug and then fixed in $10 \%$ formalin for 24 hours. Fixed samples were dehydrated in graded ethanol (EtOH) series $(70 \%(\mathrm{v} / \mathrm{v})$ to $100 \%(\mathrm{v} / \mathrm{v}) \mathrm{EtOH})$. Dehydrated samples were infiltrated with molten paraffin at $56^{\circ} \mathrm{C}$ for 2 hours and embedded into paraffin blocks. Thin sections of thickness 5-10 $\mu \mathrm{m}$ were obtained using a rotary microtome. Sections were stretched on gelatin coated microscope slides and allowed to attach at $50^{\circ} \mathrm{C}$ for 1 minute.

Sections were stained with hematoxylin (Electron Microscopy Sciences) for 20 minutes followed by differentiation in $1 \%$ acid alcohol and the contrast of hematoxylin was enhanced by a bluing agent $(0.2 \%$ ammonia). Eosin-staining (Electron Microscopy Sciences) was done by immersing the slides in eosin for 2 minutes. Sections were mounted using Permount ${ }^{\circledR}$ and were viewed with an Olympus-IX81 microscope.

Scanning Electron Microscopy: Spheroids were fixed in 2.5\% glutaraldehyde and post fixed in osmium tetraoxide. Following the same protocol as mentioned above for histology, the samples for SEM were dehydrated. After dehydrating with $100 \% \mathrm{EtOH}$, spheroids were critically dried using hexadimethyldisilazane on a lysine coated glass coverslip. The coverslip with the spheroid was mounted onto SEM stubs using doublesided carbon tape and sputter coated with gold palladium for 2 minutes under argon atmosphere. Samples were observed with a JEOL 1500 -E scanning electron microscope operating at an accelerating voltage of 20 Kilovolts. 
1.2.4 Cytotoxicity assessment under Doxorubicin chemotherapy and adjuvant hyperthermia

The effect of the following treatments was studied in Skov-3 and Dx5 spheroids; 1) Doxorubicin (DOX), and 2) Combination of Doxorubicin and slow rate Hyperthermia (@ $\left.43^{\circ} \mathrm{C}\right)(\mathrm{DOX}+\mathrm{HYP})$ in a cell culture incubator set to $43^{\circ} \mathrm{C}$. For DOX, cell media containing $12.5 \mu \mathrm{M}$ DOX-HCl was prepared and $200 \mu \mathrm{l}$ was dispensed into each well of a 96 well plate. Spheroids were transferred into this media (equivalent DOX concentration: $10 \mu \mathrm{M}$ ) and the plates were incubated under standard conditions (Day 0). Hyperthermia was simulated in a cell-culture incubator (CellStar $\left.{ }^{\circledR}\right)$ that was set to $43^{\circ} \mathrm{C}$. Spheroids were transferred into DOX-HCl containing media and incubated at $43^{\circ} \mathrm{C}$ for 1 hour after which, they were returned to the $37^{\circ} \mathrm{C}$ incubator (Day 0). Every 24 hours following Day 0, half of the media from each well was replaced with fresh media containing DOX. Spheroid volume and the number of live cells were chosen as the measures to assess the spheroid's response to the above mentioned treatments.

Spheroids were monitored daily after day 0 until day 6 using an Olympus-IX81 inverted microscope. Images were collected under bright-field and the major and minor diameters of the spheroid were measured in Image $\left({ }^{\circledR}\right.$ software (NIH) using a slight

$$
\pi \times \frac{\left(\text { Minor Diameter }{ }^{2}\right) \times(\text { Major Diameter })}{6}
$$

modification of procedure suggested by Rodday et.al (Rodday et al., 2011). From the measured diameters, volume was determined using the formula in Equation 1 
To assess the cytotoxicity induced by the above treatments an Alamar Blue assay was performed on Skov-3 and MES-SA/Dx5 spheroids on Day 6 according to the manufacturer's instructions. Alamar Blue is a commercially available dye that is routinely used to study cell growth and function in mono-layer cultures. Specifically, it measures the metabolic activity of cells through the conversion of nonfluorescent resazurin to a highly fluorescent form, resorufin $\left(\lambda_{\mathrm{ex}}: 530 \mathrm{~nm} ; \lambda_{\mathrm{em}}: 570 \mathrm{~nm}\right)$ inside the cells. Cells that are dead cannot convert resazurin to resorufin. Fluorescence from resorufin is directly proportional to the number of live cells. Spheroids were incubated with Alamar Blue for 10 hours and the fluorescence was measured in a Tecan ${ }^{\circledR}$ microplate reader. The fluorescence values were converted to number of cells using a calibration curve (number of cells vs Alamar Blue fluorescence) that was obtained in monolayer.

\subsection{Results and Discussion}

\subsubsection{Spheroid formation.}

The type of agarose plays a critical role in liquid overlay cultures for spheroid formation. In our case, only electrophoretic quality agarose for DNA separation prepared in PBS resulted in optimal spheroid ${ }^{2}$ generation. Using other low grade agarose and gels prepared in DI water or McCoy's 5A media (with/without FBS) did not result in the formation of spheroids. Similarly, agarose coating had to be done freshly before every spheroid culture. Storing the agarose coated plates at $4^{\circ} \mathrm{C} /$ Room temperature $\left(23^{\circ} \mathrm{C}\right)$ and

\footnotetext{
${ }^{2}$ Optimal spheroid: A dense spherical structure in which individual cell boundaries are indistinguishable.
} 
using them for spheroid culture or utilizing the same batch of agarose repeatedly for multiple cultures did not result in optimal spheroid formation. This is due to the tendency of agarose to gradually lose moisture which has been shown to affect spheroid formation (Friedrich et al., 2009). Therefore, it is recommended to aliquot the agarose gels after preparation and use the aliquots for coating the plates. Alternatively, the gel coated plates can be stored in a hermetically sealed chamber, if one is available.

Physical models, such as the Gompretzian kinetics (bi-phasic) model for tumor growth and the Smoluchowski rate equations for self- assembly have been used to explain the formation and growth of tumor spheroids (Enmon et al., 2001; Enmon et al., 2002; Olea et al., 1994). In general, spheroid formation in liquid overlay culture progresses through the formation of cell clusters followed by the transient redistribution of clusters, aggregation and finally the compaction of aggregates. Figure. 1 shows different stages of spheroid formation from Skov-3 cells. On initial seeding, cells settle down under the influence of gravity and form clusters. Clustering is solely dependent on the initial density of cell seeding, random cell placement and the cell-cell distance. The aggregation of clusters happens due to random collisions (cell-cell, cell-cluster) in the culture as predicted by Brownian dynamics ${ }^{3}$. Furthermore, biologically attractive forces (Froehlich type forces; (Rowlands et al., 1981)) that are cell potential dependent and act over short distances (one cell distance) have been postulated to play a role in transient redistribution and subsequent aggregation. Although, physical forces lead to the initial self-assembly of cells, the final

\footnotetext{
${ }^{3}$ The reader is referred to papers from (Enmon et al., 2002) for more details on physical models of spheroid formation
} 
stages of spheroid formation, i.e., compaction of aggregates through the secretion of ECM proteins, viz collagen, fibronectin and intra-spheroid cell growth, is dictated by the type and characteristics of the cells. In our case, MES-SA uterine sarcoma cells did not form compact spheroids and only formed loose cell aggregates that dissociated under small shear forces (i.e., agitation of culture plates, pipetting inside the well). MES-SA/Dx5 exhibit differential expression of 37 proteins compared to MES-SA cells which might explain the difference in spheroid formation ability among them (Hsinchu et.al, 2013 (explained in detail below).
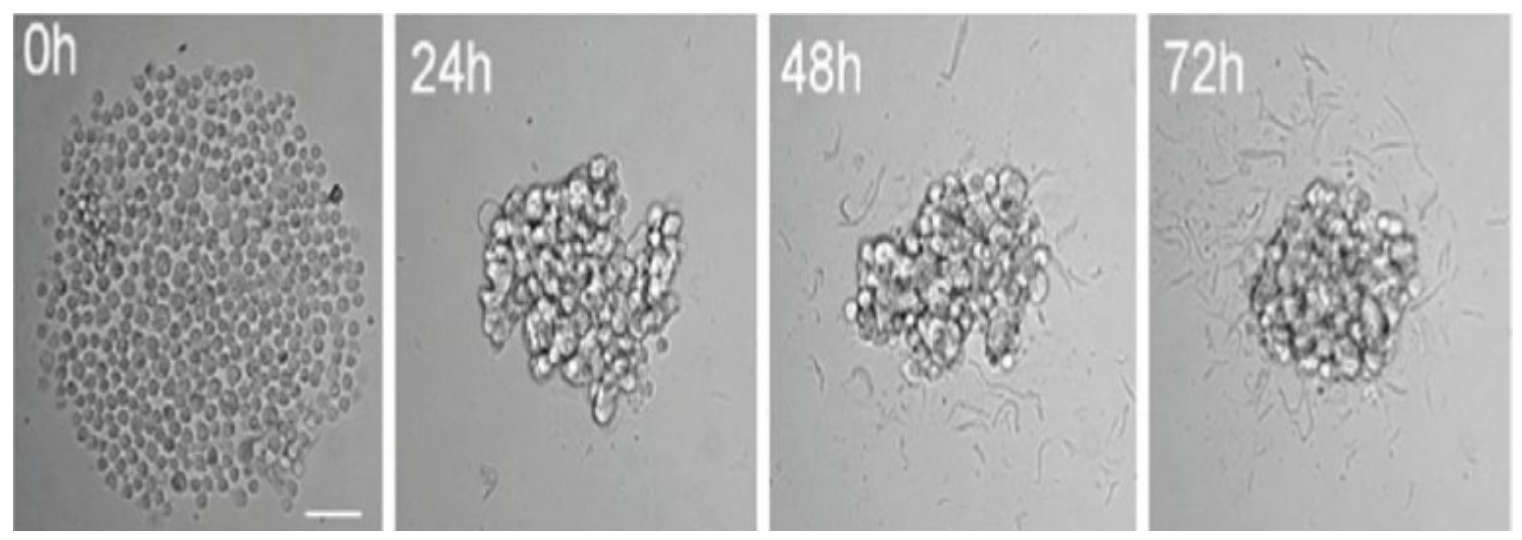

Figure 1.1 : Stages of Spheroid Formation a) initial clustering, b) reorganization of clusters, c) aggregation, d) compaction. Scale bar is $100 \mu \mathrm{m}$. Reproduced with permission from Pampaloni et.al. 2013.

During spheroid formation, cells secrete or regulate proteins such as cytokeratins, vimentins (cell motility and contractility), and several cell-cell/cell-ECM adhesion markers such as E-cadherins, $N$-cadherins, and $\beta 1$-integrins. The upregulation of $\beta 1$-integrins and E-cadherins has been shown to promote spheroid compaction in hepatoma and ovarian cancer cell lines that otherwise form loose aggregates (Casey et al., 2001; Lin et al., 2006). 
However, the secretion of these markers does not bear any direct relation to compact spheroid formation. Spheroid compaction is strongly dependent on the cell and culture type. Skov-3 cells cultured in hanging drop cultures did not form spheroids, whereas, they formed spheroids when cultured in liquid overlay (Sodek et al., 2009). Furthermore, in a study by Lee et al. investigating the differences between the 2-D and 3-D culture of several (31) epithelial ovarian cancer cell lines, $70 \%$ of the cells that formed compact spheroids (6 of 8 , including Skov-3) showed an upregulation in cytokeratin compared to cells in monolayers (Lee et al., 2013). Interestingly, the expression of E-cadherin and N-cadherin was not found in either Skov-3 spheroids or 2-D cultures. Similarly, upregulation of $\beta 1$ integrin through ectopic expression did not result in the compaction of Skov-3 aggregates grown in hanging drop culture (Sodek et al., 2009).

The factors that seem to be fair predictors of spheroid formation are the adhesion/invasion properties and the innate ability of the cells to synthesize ECM molecules in relation to their culture environment. Cells that formed spheroids synthesized ECM in greater amounts compared to cells that did not form spheroids (Lin et al., 2006). Moreover, the exogenous addition of ECM components such as collagen and fibronectin to the spheroid culture can either deter or promote spheroid formation. This is because ECM fibers present sites for cell attachment and growth which leads to spheroid formation or increase the distance between cells thereby reducing cell-cell interactions necessary for

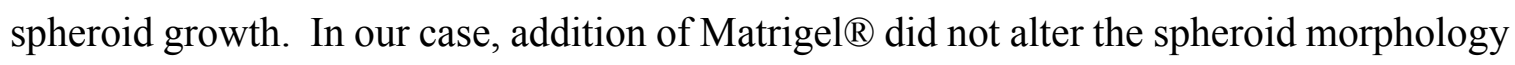
but accelerated the spheroid formation in Skov-3 and MES-SA/Dx5 cells. However, MESSA cells did not form spheroids despite the addition of Matrigel ${ }^{\circledR}$. 


\subsubsection{Spheroid characterization.}

Figure 1.2 shows the bright field images (Obj 4X) of Day 5 spheroids formed from MES-SA/Dx5 and Skov-3 cells. It is evident that Skov-3 cells form small and compact spheroids whereas, MES-SA/Dx5 cells form large spheroids with independent cells visible around their circumference (Figure1.2). The volume of MES-SA/Dx5 spheroids was $0.342 \pm 0.012 \mathrm{~mm}^{3}$ (mean \pm S.E. of $\mathrm{n}=5$ spheroids) and that of Skov- 3 was $0.083 \pm 0.010$ $\mathrm{mm}^{3}$ (mean \pm S.E. of $\mathrm{n}=5$ spheroids). The large size of MES-SA/Dx 5 spheroids compared to Skov-3 spheroids might be due to faster proliferation of MES-SA/Dx 5 cells (doubling half-life, $\mathrm{t}_{1 / 2}=17$ hours) compared to Skov-3 cells ( $\mathrm{t}_{1 / 2}=43$ hours). This is supported by the results of the Alamar Blue assay which showed that Skov-3 spheroids had a live cell count of $26,000 \pm 1500$ cells (mean \pm S.E. of $n=3$ ) and MES-SA/Dx 5 spheroids consisted of $52,000 \pm 3,000($ mean \pm S.E. of $n=3)$.

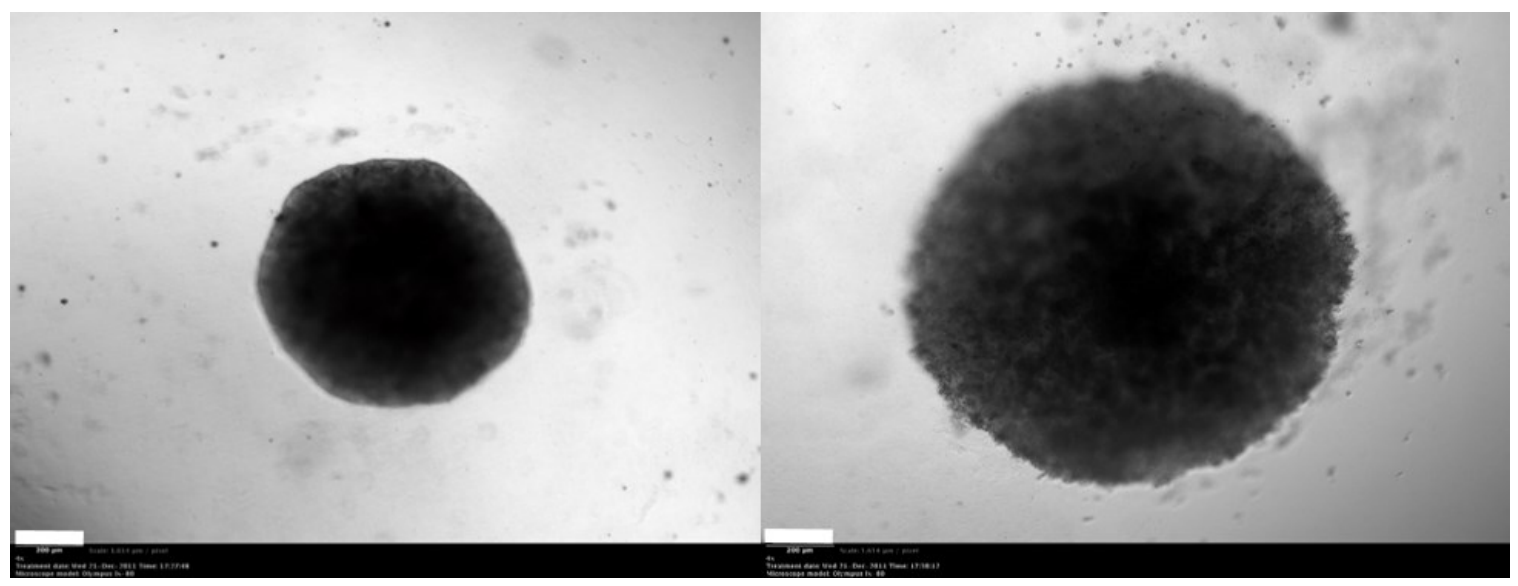

Figure 1.2: Brightfield images of spheroids. Images were taken using 4X objective, Skov3 (left) and Dx-5 (right). Scale bar at the bottom left of images is $200 \mu \mathrm{m}$. 
Scanning electron microscope images reveal striking differences between MESSA/Dx5 and Skov-3 spheroids and confirm the inferences drawn from bright-field images. The 3-D organization of cells is evident from the SEM images at low magnification. Skov3 spheroids form ellipsoid structures with small circular openings on the surface and individual cells are indistinguishable due to the dense ECM matrix synthesized by the cells (Figure 1.3). Similar morphology due to the secretion of ECM was observed in hepatocyte spheroids (Kelm et al., 2003; Peshwa et al., 1996). Alternately, high magnification images of MES-SA/Dx 5 spheroids show a porous structure and individual cells held together by the membrane inter-digitations of the cells (Figure 1.4). Faute et al. also observed similar differences in the cellular organization in tumor spheroids formed from Adriamycin resistant and Adriamycin sensitive breast cancer cell lines MCF-7 (dit Faute et al., 2002). The resistant cell line showed a lack of e-cadherin expression between the cells and the spheroids resembled an aggregate of cells held together by the membrane inter-digitations on cell membrane. Moreover, studies have shown that as the tumors increase in size, the ECM disintegrates due to reduction in collagen fiber thickness. This explains the loose association of cells in MES-SA/Dx5 spheroids. Therefore, based on the cellular organization, MES-SA/Dx5 spheroids resemble large cell aggregates (LA's) and Skov-3 spheroids represent compact/optimal spheroids. 

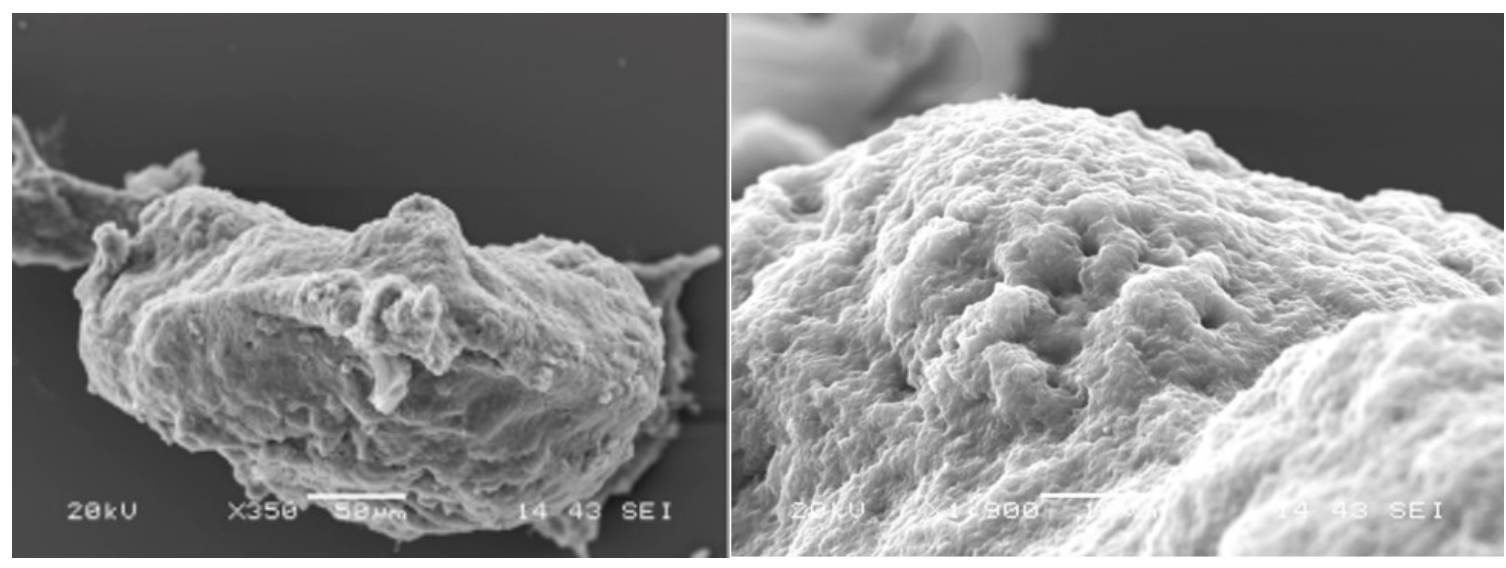

Figure 1.3: SEM Images of Skov-3 Spheroids show compact organization, 350X magnification (left) and 1,900X magnification showing the surface (right)

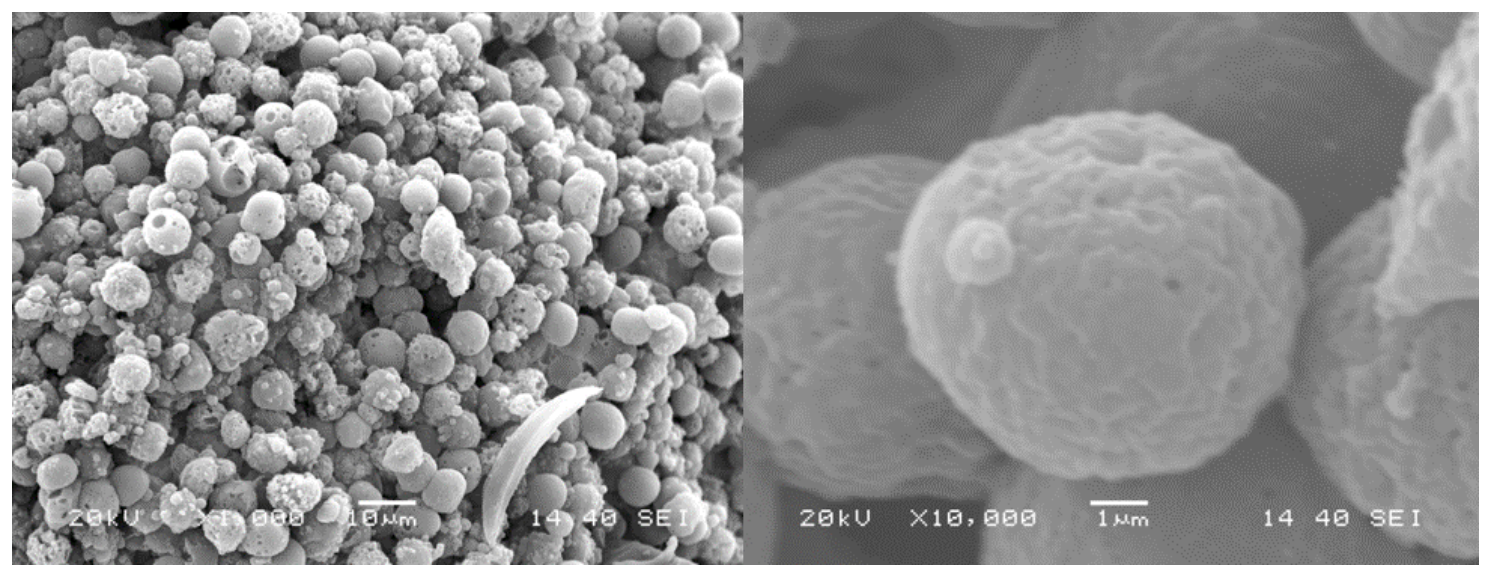

Figure 1.4: SEM Images of MES-SA/Dx-5 Spheroids show distinct cell bodies. 1000X magnification (left) showing disperse cellular organization and 10,000X magnification (right) showing the individual arrangement of cells. Visible perforations on the cell bodies may have been due to tissue processing.

Histology sections show a stratified organization of cell layers, i.e., a necrotic core surrounded by a dense cuticular layer of proliferating cells (Figure 1.5). Limited molecule 
diffusion results in a significant deprivation of nutrients and oxygen at the core of the spheroid. This, coupled with the accumulation of waste products due to cell metabolism, results in a necrotic core (Figure 1.5). The availability of nutrients and oxygen at the core, with an increase in distance from the periphery, is determined by cell consumption rate and the presence of an ECM. The necrotic core is surrounded by a quiescent cell region in which cells are metabolically dormant and do not reproduce due to a lack of nutrients. In some cases, a large fraction of cells separated from the quiescent region has been shown to regain their reproductive ability. In spheroids, these cells can be recruited for repopulating the proliferating cells at the spheroid periphery.

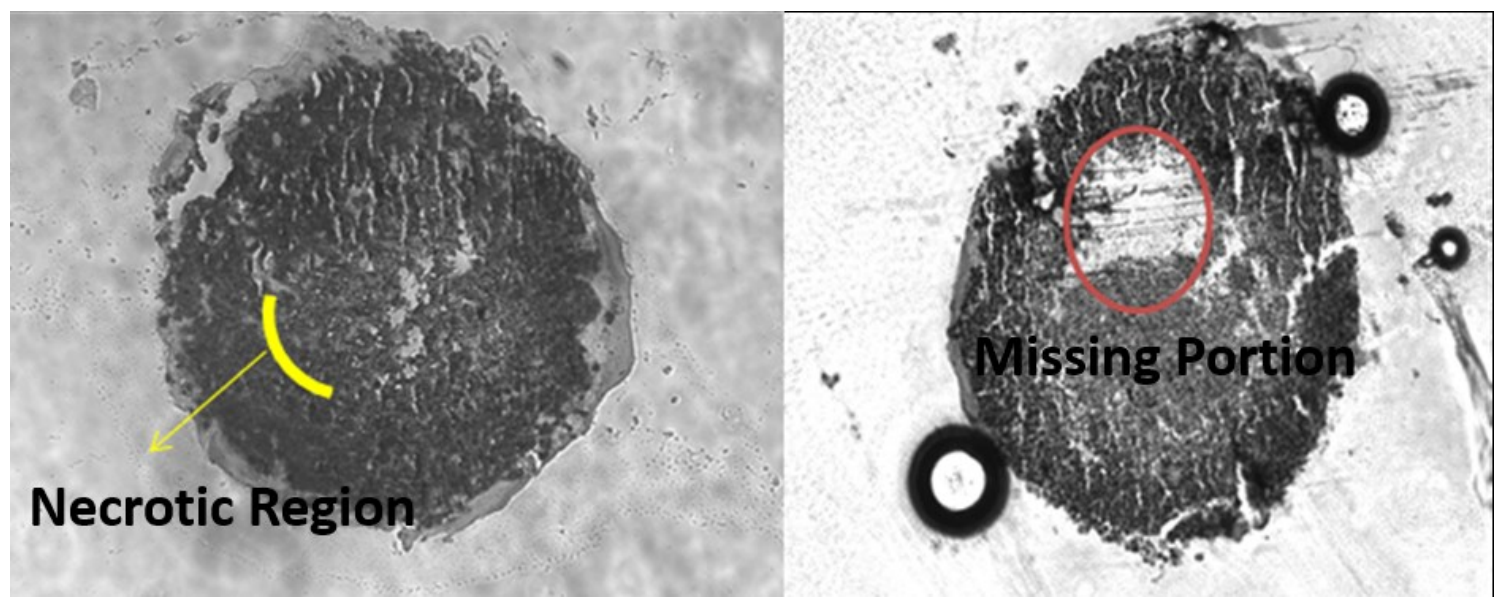

Figure 1.5: Histological analysis of Skov-3 (left) and MES-SA/Dx-5 (right) spheroids show the stratified organization of the spheroids. Spheroids show a dense cuticular layer (outside) followed by a lighter core containing necrotic cells (lighter appearance and shrunk cell nuclei). The empty spaces in the images are due to the loss in sample during histological processing.

Steep gradients of oxygen and $\mathrm{pH}$, which are in agreement with theoretical calculations, have been found in spheroids through microelectrode measurements. Experiments in HT29 colon carcinoma spheroids $(\sim 1 \mathrm{~mm}$ in diameter) showed that the 
oxygen partial pressure $\left(\mathrm{pO}_{2}\right)$ decreased by $70 \%$ at a distance of $500 \mu \mathrm{m}$ from the spheroid periphery and $\mathrm{pO}_{2}$ levels were at $0 \mathrm{mmHg}$ at the core (Acker et al., 1987; Carlsson and Acker, 1988; Sutherland, 1988). The accumulation of metabolites inside the spheroid results in regions of lower $\mathrm{pH}$, which can vary between 5.8 and 7.4 depending on the type of spheroid. The structural characteristics and resulting gradients of $\mathrm{O}_{2}$ and $\mathrm{pH}$ have important implications on the therapeutic response of cells in spheroids to DOX and adjuvant hyperthermia treatment.

\subsubsection{Response to Doxorubicin and adjuvant hyperthermia treatment.}

The cytotoxic effect of DOX and DOX + HYP treatment was evident after day 1 from the dissociation of cells at the spheroid periphery (Figure 1.6). The dissociation of cells occurred due to the loss of cell-cell contacts and cell-ECM contacts caused by cell death. That the dissociated cells were dead was confirmed by that fact that seeding them in a separate 96 well plate did not result in colony formation or cell- attachment and growth. The reduction in cell viability was also later confirmed using the Alamar Blue assay. At the $6^{\text {th }}$ day, a spheroid with reduced volume is visible (Figure 1.6). A consistent decrease of volume in Skov-3 spheroids was observed after day 2 in both DOX and DOX+HYP groups while the volume increased in the control group. This response is slower than that observed in monolayer cultures and is probably due to the relatively slow diffusion of DOX into the tumor spheroid. Doxorubicin and mitoxantrone bind avidly to cell-DNA and have been shown to only slowly penetrate tumor spheroids (Tredan et al., 2007b). On day 6, there was no significant difference in the reduction of volumes between DOX and 
$\mathrm{DOX}+\mathrm{HYP}$ treatments. A student's t-test was performed to assess the statistical significance.

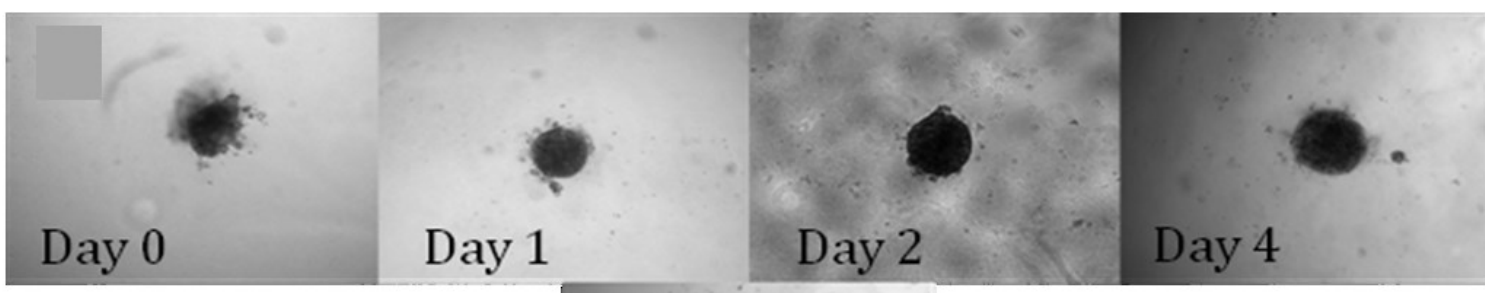

\section{Skov-3 Control}

\section{Day 6}

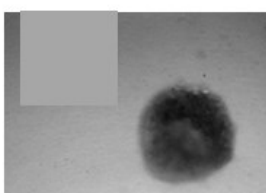

Day 0

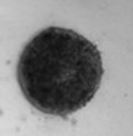

\section{1}

1

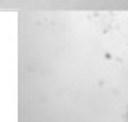

\section{Skov-3 Treatment}

\section{Day 6}

Figure 1.6: DOX+HYP therapy causes cell death at the spheroid periphery. (Top) Skov-3 control spheroids without any treatment, the growth of the spheroid over time is evident, (Bottom) Skov-3 Spheroids under DOX treatment, images show a shrinking spheroid with progress of time. Scale bar represents $100 \mu \mathrm{m}$.

Similarly, MES-SA/DX5 LA's exhibited a decrease in volume until day 3 of treatment, after-which there was a minor increase in their volumes, possibly due to higher cell multiplication (Figure 1.7). The control group of MES-SA/Dx5 LA's also showed a decrease in their volume after day 4 . This might be due to the rapid depletion of nutrients 
in media owing to their big size and a higher cell number in the aggregate. There was no significant difference between the volume decrease in DOX and DOX + HYP groups in MES-SA/Dx5. The spheroid cytotoxicity assay showed the presence of viable cells and an intact spheroid mass on day 6 . These results show that the spheroids might be resistant to a combination of DOX and adjuvant hyperthermia.

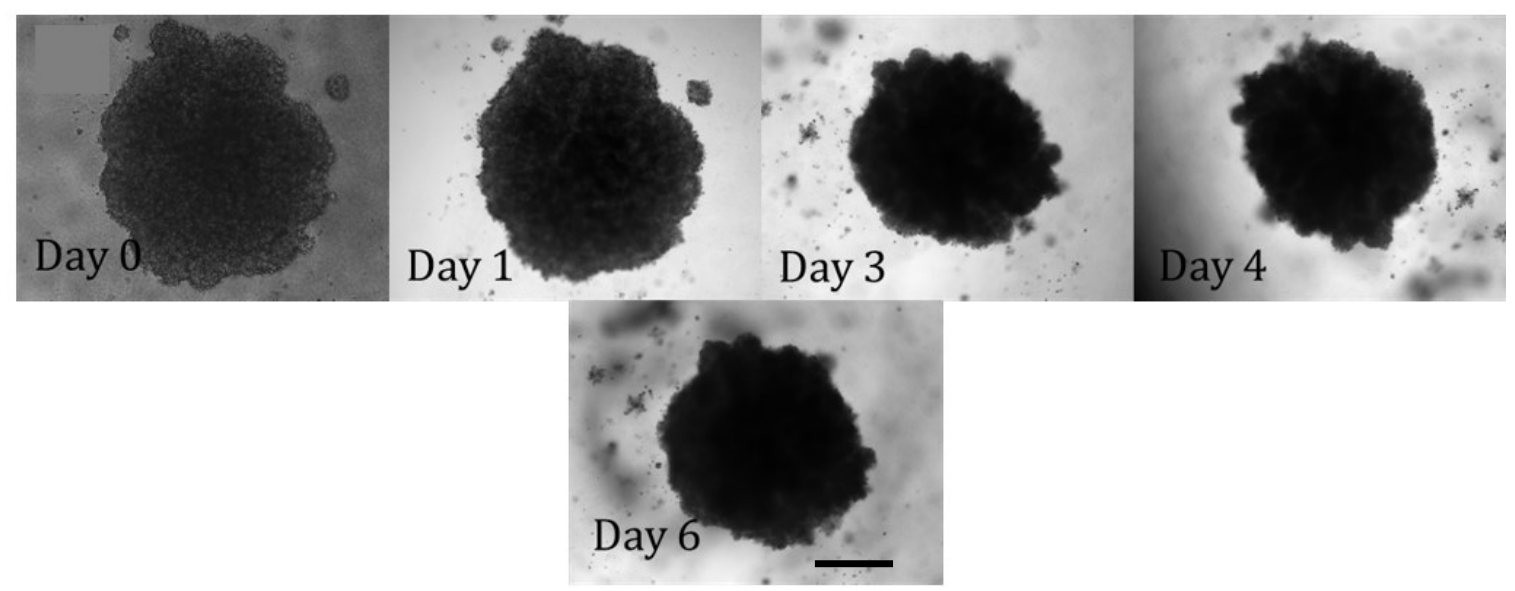

Figure 1.7: MES-SA/Dx-5 spheroids under combined DOX + HYP treatment over 6 days. Scale bar represents $200 \mu \mathrm{m}$.
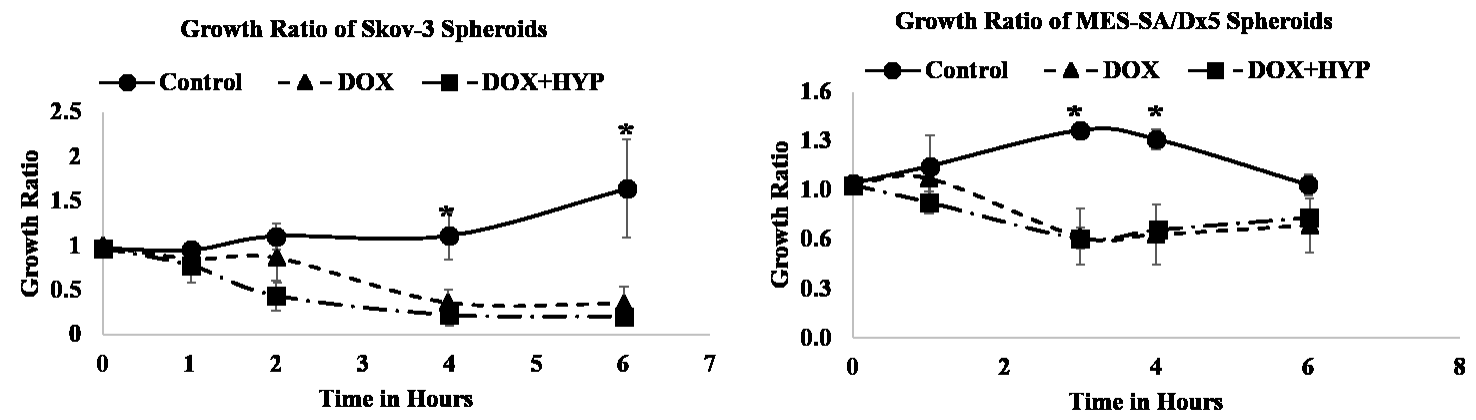

Figure 1.8: Growth Curves of Skov-3 (left) and MES-SA/Dx 5 spheroids show reduction in spheroid volume. Data is represented as mean \pm stdev of $n=4, *$ indicates significant difference $(p<0.05)$ between control and treatment. The growth ratios were calculated by dividing the corresponding day volume by the initial volume of the spheroid before treatment. 


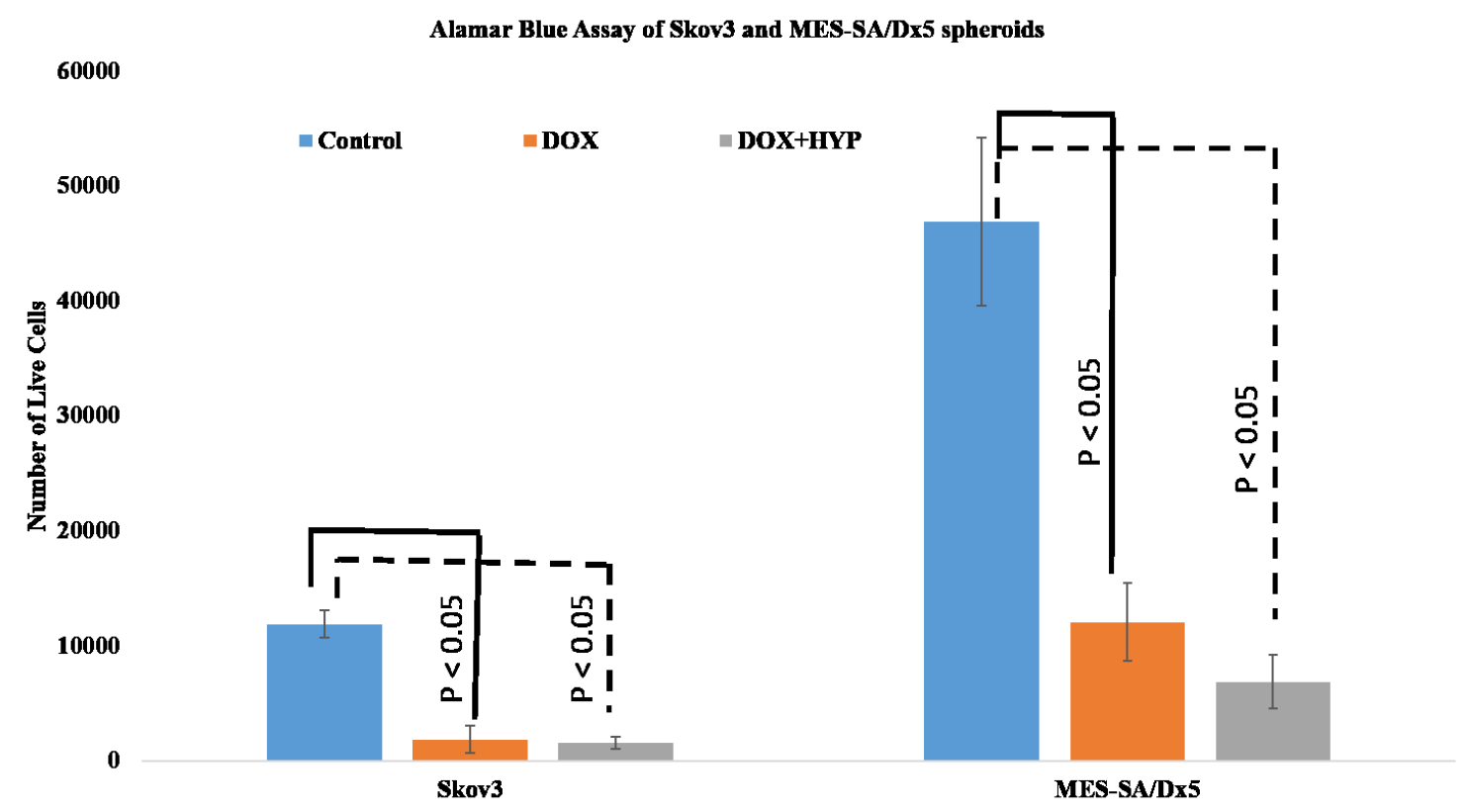

Figure 1.8:Alamar blue assay results showing the presence of viable cells at the end of treatment i.e., day 6. $\mathrm{P}<0.05$ indicates significant difference between the control and different treatments. No significance was found for mean cell viability $(n=6)$ between DOX and DOX +HYP treatment.

Tumor cells respond to a hypoxic environment by expressing Hypoxia Inducible Factor 1 (HIF-1). HIF-1 binds to the DNA sequence 5'-RCGTG-3' and up-regulates genes for vascular endothelial growth factor (VEGF) and angiopoetin-2, precursors for promoting angiogenesis. Rice et al. have shown that in Chinese hamster ovary cells subjected to hypoxia, DNA synthesis is inhibited. However, when the cells are returned to normoxic conditions, certain populations of cells (nearly $60 \%$ ) show over-replication of DNA that is responsible for amplification of dihydrofolate reductase gene and subsequent resistance to the drug methotrexate. Similarly, these authors have shown that the combination of methotrexate and doxorubicin therapy in Chinese hamster ovary cells under hypoxia resulted in a 10-100-fold increase in the frequency of drug resistance when 
compared to independent treatments. Methotrexate resistance was mediated by amplification of the dihydrofolate reductase gene and doxorubicin resistance was a result of up-regulation of P-glycoprotein (Rice et al., 1986; Rice et al., 1987). In a study by Comerford et al. PCR analysis confirmed a 7-fold increase in MDR in epithelial cells under hypoxia. The studies mentioned above confirm the role of hypoxia in inducing drug resistance. In a hypoxic environment, glucose metabolism by the glycolytic pyruvate pathway produces lactate, and as a result, the extracellular $\mathrm{pH}$ in most tumors tends to be acidic (Dang and Semenza, 1999; Tredan et al., 2007b). However, the intracellular pH is maintained at a normal level ( $\mathrm{pH} 7.2-7.4)$. Weakly acidic drugs can permeate the cells in their uncharged state, which upon entry into the cells become protonated at neutral $\mathrm{pH}$ and get trapped in cellular organelles. This leads to higher intracellular accumulation of weakly acidic drugs (Tredan et al., 2007b). The acidic environment is a hindrance to weakly basic drugs such as doxorubicin. Gerweck et al. have shown that lowering the tumor $\mathrm{pH}$ (by 0.2) by infusion of glucose lead to a 2-fold increase in intracellular accumulation of chloroambucil (weak acid) and a 2-fold decrease in accumulation of doxorubicin (weak base). Tumors treated with doxorubicin and chloroambucil under glucose infusion showed opposite effects in growth delays (Gerweck et al., 2006) ${ }^{4}$. In our case, from the spheroid organization, it is apparent that gradients of Oxygen as well as $\mathrm{pH}$ may exist in Skov-3 and MES-SA/Dx5 spheroids. Furthermore, we can speculate that these gradients will be steeper in Skov-3 spheroids compared to MES-SA/Dx5 LA's. Therefore, the acidic environment

${ }^{4}$ For a detailed analysis of drugs and their response to extracellular $\mathrm{pH}$ the reader is referred to Raghunand et al (Mahoney et al., 2003; Raghunand et al., 2003; Raghunand et al., 1999). 
inside the Skov-3 spheroid will be a hindrance to the transport and cellular uptake of Doxorubicin. In MES-SA/Dx5 LAs the amplified P-gp mediated drug resistance owing to a hypoxic environment inside the LA (explained below) may also contribute to Doxorubicin resistance. Further studies are needed to confirm this.

Tumors have higher uptake of glucose compared to normal tissues due to the upregulation of glucose hexokinase-2. This leads to higher glucose metabolism and glucose transport flux in tumors (Dang and Semenza, 1999). Glucose depletion due to nutrient deprivation leads to an increase in p-glycoprotein expression (Ledoux et al., 2003; Yun et al., 1995). Studies by Yun et al. (Yun et al., 1995) have shown that glucose regulated stresses confers drug resistance in human cancer cells by down-regulation of DNA topo2. In addition to the above-mentioned mechanisms, tight aggregation of cells in spheroids gives rise to new (de-novo) forms of drug resistance as a result of 'contact effect' (Sutherland, 1988). These mechanisms include: alterations in drug targets, reduction in cancer cell apoptosis, decrease in cell proliferation and an integrin signaling cascade.

The proliferating rim of the spheroids is a primary barrier that hinders drug diffusion towards the core and since cells in this region are metabolically active they rapidly consume the drug, which explains cell detachment due to cell death at the periphery. The effect of this physical barrier is likely to be more pronounced in Skov-3 spheroids than in MES-SA/Dx5 due to their compact aggregation. The slow transport of DNA binding drugs like DOX coupled with the dense ECM of Skov-3 spheroids, results in an inhomogeneous distribution of DOX in the interior regions, thereby, mitigating the augmentation of its cytotoxic effect with adjuvant hyperthermia. Treatment with 
topoisomerase II inhibitors has been shown to increase thermotolerance and might contribute towards resistance to combinational therapy.

Early studies in spheroids grown from EMT6 (mice mammary carcinoma; Sutherland et.al) and V79 (Chinese hamster ovary; Olive et al.) illustrate different mechanisms of contact resistance (Olive and Durand, 1994; Sutherland, 1988). These include changes in cell cycle and chromatin packaging, differences in cell shape and changes in repair related gene expressions. Chromatin packaging can play an important role in DNA damage and repair by influencing the nature of DNA lesions caused in response to therapy. DNA extraction and denaturation studies showed that DNA in spheroids is more resistant to unwinding than cells in monolayers. Therefore, the regions of DNA that are sensitive to respective therapeutic moiety/modality may not be targeted and denaturation of DNA may not occur due to tight packaging of the chromatin. Cells in spheroids assume a different shape than those in monolayers. The changes in microtubule arrangement may increase resistance to heat by changing the membrane properties and also influences the uptake configuration of a respective cell. Cells treated with microtubule inhibitors showed increased etoposide (topoisomerase II inhibitor) accumulation and DNA damage (Yalowich, 1987). Monolayer cells grown in spheroid cultures also exhibit fast DNA repair (G2 cycle) to damage induced by ionizing radiation. Furthermore, EMT6 mouse mammary carcinoma cells grown as compact spheroids showed an inability to arrest in the $\mathrm{G} 2 / \mathrm{m}^{5}$ phase of cell division on exposure to cell mitosis inhibitor 4-

\footnotetext{
${ }^{5} \mathrm{G} 2 / \mathrm{M}$ is the final stage in cell cycle where damages to DNA replicated in the S-phase are verified and is followed by the prophase of cell mitotic division.
} 
hydroperoxycyclophosphamide (4-HC). This was not observed in monolayer cultures which showed $\mathrm{G} 2 / \mathrm{m}$ arrest followed by cell death. The degree of compaction also plays an important role in DNA repair. Significant differences in DNA replication and cell cycles were observed by Croix et al. between loose spheroids and compact spheroids. These observations explain the trends seen for the growth curves under DOX therapy in Skov-3 spheroids and MES-SA/Dx5 LAs. DOX is a DNA intercalating agent which arrests cell replication via topioisomerase II inhibition in the late S-phase of cell division that immediately precedes the G2 cycle. Additionally, the tight chromatin packaging may not present sites on the DNA for DOX binding. This coupled with the inability of spheroids to arrest in the $\mathrm{G} 2 / \mathrm{m}$ phase explains the contact induced drug resistance to DOX. In order to resolve contact mediated resistance, the cellular organization of spheroids needs to be disrupted. This can be done via the use of collagenase that can disrupt the tumor ECM, cell penetrating peptides and or via external stimuli such as (ultrasound)

In MES-SA/Dx5 LA's, expression of DOX and heat resistance related genes also play an important role towards resistance to DOX and hyperthermia. MDR mediated by Pglycoprotein and an increase in thermotolerance and upregulation of Heat Shock Protein70 may be the determinants of observed resistance to DOX $+\mathrm{HYP}$ treatment. Increase is Pgp expression was observed in the quiescent regions of DU-145 prostate cancer spheroids which resulted in the exclusion of DOX from the cells in the spheroid and reduced uptake of DOX in the deeper regions of spheroid. Incubation with MDR reversal agents such as verapamil increased the DOX accumulation in spheroids (Wartenberg et al., 1998). Since quiescent cell regions are developed in relation to spheroid size, large tumor spheroids are 
more likely to exhibit an increase in P-gp resistance compared to small tumor spheroids. Studies in DU-145 prostate cancer spheroids by Wartenberg et al. have found that upregulation of P-gp expression under hyperthermia is not correlated with a corresponding increase in HSP expression. Instead, hyperthermia stabilized the HIF-1 $\alpha$ expression which increased its production and increased the P-gp expression. Moreover, high levels of reactive oxygen species (ROS) produced during DOX treatment and hyperthermia increased the P-gp expression (Wartenberg et al., 1998; Wartenberg et al., 2005). An increase in production of HIF-1 $\alpha$ and ROS under incubator hyperthermia in MES-SA/Dx5 monolayers has been verified in our laboratory by Lei et al. (Lei et al., 2014). A minor increase in MES-SA/Dx5 LA's after day 3 suggests an increase in cell multiplication to balance cell death caused by DOX and DOX + HYP. It is possible that cells from quiescent layers are recruited to repopulate the dead cell population. This kinetic change in cell multiplication was observed by Olive et al. in DOX treated spheroids (Olive and Durand, 1994). The above results illustrate the multifactorial nature of drug resistance (genetic \& kinetic) which present a different perspective to study the tumor interaction to therapy that is otherwise not achieved by mono-layer cultures. The results from spheroid growth and cytotoxicity to DOX and DOX $+\mathrm{HYP}$ also confirm the first hypothesis that spheroids are resistant to combination of DOX and slow rate adjuvant hyperthermia. Previous experiments in MES-SA/Dx5 showed that the combination of DOX and incubator hyperthermia resulted in significantly more cell killing ( $80 \%$ growth inhibition) compared to DOX therapy alone ( $40 \%$ growth inhibition). The results above show that the successive application of DOX and adjuvant hyperthermia may not be successful in achieving increased cell killing than DOX therapy alone in multicellular spheroids. Therefore, in 
order to achieve the additive advantage of both the therapies in tumor spheroids the drug resistance mechanisms need to be circumvented. P-gp MDR can be circumvented by encapsulating the drug in nanoparticles and the drug can be released in a controlled manner from the nanoparticles. Controlled release of drug may also have an indirect advantage in avoiding the drug tolerance induced by the expression of HSP70. Studies have shown that HSP70 levels in bovine aortic endothelial cells peaked 2 hours after the removal of heat exposure ( $\left(30\right.$ mins at $43^{\circ} \mathrm{C}$ ) followed by a gradual decline to base level and another second peak at 12 hours (Wang et al., 2003; Wang et al., 2008). Hence, if the expression kinetics of HSP70 is known then drug release from the nanoparticles can be controlled to avoid thermal and drug tolerance to achieve the additive effect of both therapies on the cancer cell. Another strategy to avoid the expression of HSP70 is by using short duration rapid rate hyperthermia. This can be easily achieved by using NIR dyes such as IR820 and ICG that generate heat on exposure to NIR laser. However, owing to the short plasma life and rapid degradation of these dyes in-vivo they have to be delivered shielded in nanoparticles.

\subsection{Is slow diffusion a hindrance to therapy?}

The above discussion on limited penetration and drug resistance may seem to present an overly complex challenge for achieving the benefits of DOX and adjuvant hyperthermia. However, slow diffusion provides another perspective for tumor therapy. If the drug is slow to diffuse inward, it will also be slow to diffuse outward and coupled with the active exclusion of drugs from cells will increase the intratumoral concentration of drug. One of the strategies to increase efficacy of chemotherapeutics is to effectively deliver the drug (without degradation or in-vivo clearance) by exploiting the poorly 
developed vasculature of tumors and increase the intratumoral concentration of drug. At the same time, drug resistance should also be circumvented. This can be effectively done without the systemic toxicity associated with the free form of drugs by encapsulating them in nano carriers. Once the nanoparticles reach the tumor site or penetrate the tumor, other aspects of therapy, like the dosage and timing of hyperthermia, can be planned to achieve the combined effect of the therapies (drug and adjuvant hyperthermia) on cell killing. Hyperthermia has been shown to enhance drug delivery to tumors by increasing local blood pressure and promoting drug extravasation at the hyperthermia site. For drug delivery, hyperthermia is preferable to tumor ablation as ablation leads to collapse of blood vessels and impedes drug delivery. Although there are several methods of hyperthermia delivery, NIR Dyes (600-1000 nm wavelength) such as Indocyanine Green and IR820 that generate heat rapidly on exposure to laser energy can be used to deliver localized hyperthermia at the tumor site. Combined with the fluorescence of these dyes in the NIR window where absorbance and scattering by in-vivo tissues is minimal, ICG and IR820 serve as ideal probes for image guided chemotherapy with adjuvant hyperthermia. However, their direct administration in-vivo is challenged by a lack of stability, i.e., rapid degradation and fast clearance from the plasma (Fernandez-Fernandez et al., 2012; Saxena et al., 2004). These shortcomings can be overcome by delivering them entrapped in a nano carrier. However, nanoparticles also present the challenge of limited penetration into the tumor tissue owing to bigger size compared to free drugs or dyes. 


\subsection{Limitations of spheroid models}

Although spheroids replicate the morphological and functional features of tumor nodules, solid tumors and micro metastases, the static culture system adapted to culture the spheroids is disadvantageous and thus dynamic conditions are needed to produce welldefined models. In-vivo, tumors have multiple cell types present, such as fibroblasts, cancer stem cells, etc. Therefore, using a single cell type may not faithfully represent the functional complexity of in-vivo tumors. The co-culture of different cells into tumor spheroids and the creation of angiogenic (microvascular) networks in tumor spheroids are in their nascent stages with progress moving rapidly towards the co-culture of tumor cells with blood vessel endothelial cells, microfluidics and synthetic microvascular networks. Static culture methods for spheroids (liquid overlay, hanging drop) are not suitable for simulating dynamic conditions of macromolecule delivery, such as convection and extravasation from vasculature.

Techniques for assessing the cytotoxicity of a drug in tumor spheroids have not been standardized. Cytotoxicity assays, such as lactate dehydrogenase (LDH), 3-(4, 5dimethylthiazolyl-2)-2, 5-diphenyltetrazolium bromide (MTT), clonogenic methods and Alamar Blue applicable to cells in suspension culture have been used for spheroids. It should be noted that the use of cytotoxicity assays such as Alamar Blue and Cell Titer blue in spheroids should be considered with a careful optimization of time of incubation and spheroid type. Waltzl et al. showed that compact spheroids pose a barrier to efficient penetration of Cell Titer Blue which affects the dye availability, cell uptake and subsequent reduction. Disruption of cell-cell contacts by trypsinization or by addition of drugs 
increased the reduction of the Alamar Blue (Walzl et al., 2014). Other techniques such as acid phosphatase assay have been mentioned in the literature (Friedrich et al., 2007). However, standardized methods are still lacking and need development.

The extent of drug penetration into the spheroid can be observed in real time through confocal and two photon microscopy; however, the resolution of confocal imaging is challenging because of light absorption and scattering by the spheroid tissue. Image correction techniques (for scattering and laser power attenuation) for spheroids imaged under confocal microscopy have been described by Wartenberg et al. (Wartenberg et al., 2005). The development of confocal microscopes with smaller pinholes and sophisticated optics has led to an improvement in spheroid imaging making tumor spheroids a very valuable tool for positive/negative drug selection before in-vivo trials are undertaken.

\subsection{Conclusions.}

In this chapter, a 3-dimensional spheroid model was developed with an Ovarian carcinoma (Skov3) and uterine sarcoma (MES-SA/Dx5) cell lines. Both spheroids showed 3dimensional and stratified organization with a necrotic core (Figure $1.2 \&$ Figure 1.5). Skov-3 spheroids had a mean volume of $0.083 \mathrm{~mm}^{3}$ and MES-SA/Dx5 aggregates had a mean volume of $0.342 \mathrm{~mm}^{3}$. Scanning electron microscopy showed marked difference between the structures of Skov3 and MES-SA/Dx5 spheroids (Figure 1.3 \& Figure 1.4). Exposure to DOX with adjuvant hyperthermia (at $43^{\circ} \mathrm{C}$ for 1 hour) showed cell dissociation from the spheroid periphery (Figure 1.6). However, the combination did not result in significant enhancement in cell cytotoxicity compared to just DOX therapy alone illustrating the resistance of tumor spheroids to the combination treatment (Figure 1.8). 


\section{References}

1. Abbott, A. Cell culture: biology's new dimension. Nature 2003, 424, 870-872.

2. Acker, H.; Carlsson, J.; Mueller-Klieser, W.; Sutherland, R. M. Comparative pO2 measurements in cell spheroids cultured with different techniques. Br. J. Cancer 1987, 56, 325-327.

3. Bokhari, M.; Carnachan, R. J.; Cameron, N. R.; Przyborski, S. A. Culture of HepG2 liver cells on three dimensional polystyrene scaffolds enhances cell structure and function during toxicological challenge. J. Anat. 2007, 211, 567-576.

4. Carlsson, J.; Yuhas, J. In Liquid-overlay culture of cellular spheroids; Spheroids in Cancer Research; Springer: 1984; pp 1-23.

5. Carlsson, J.; Acker, H. Relations between $\mathrm{pH}$, oxygen partial pressure and growth in cultured cell spheroids. International journal of cancer 1988, 42, 715-720.

6. Casey, R. C.; Burleson, K. M.; Skubitz, K. M.; Pambuccian, S. E.; Oegema, T. R.; Ruff, L. E.; Skubitz, A. P. $\beta 1$-integrins regulate the formation and adhesion of ovarian carcinoma multicellular spheroids. The American journal of pathology 2001, 159, 2071-2080.

7. Cottin, S.; Ghani, K.; de Campos-Lima, P. O.; Caruso, M. Gemcitabine intercellular diffusion mediated by gap junctions: new implications for cancer therapy. Molecular cancer 2010, 9, 1 .

8. Dang, C. V.; Semenza, G. L. Oncogenic alterations of metabolism. Trends Biochem. Sci. 1999, 24, 68-72.

9. Debnath, J.; Muthuswamy, S. K.; Brugge, J. S. Morphogenesis and oncogenesis of MCF-10A mammary epithelial acini grown in three-dimensional basement membrane cultures. Methods 2003, 30, 256-268.

10. dit Faute, M. A.; Laurent, L.; Ploton, D.; Poupon, M.; Jardillier, J.; Bobichon, H. Distinctive alterations of invasiveness, drug resistance and cell-cell organization in 3D-cultures of MCF-7, a human breast cancer cell line, and its multidrug resistant variant. Clin. Exp. Metastasis 2002, 19, 161-167.

11. Enmon, R. M.; O'Connor, K. C.; Lacks, D. J.; Schwartz, D. K.; Dotson, R. S. Dynamics of spheroid self-assembly in liquid-overlay culture of DU 145 human prostate cancer cells. Biotechnol. Bioeng. 2001, 72, 579-591. 
12. Enmon, R. M.; O'Connor, K. C.; Song, H.; Lacks, D. J.; Schwartz, D. K. Aggregation kinetics of well and poorly differentiated human prostate cancer cells. Biotechnol. Bioeng. 2002, 80, 580-588.

13. Fernandez-Fernandez, A.; Manchanda, R.; Lei, T.; Carvajal, D. A.; Tang, Y.; Raza Kazmi, S. Z.; McGoron, A. J. Comparative study of the optical and heat generation properties of IR820 and indocyanine green. Molecular imaging 2012, 11, 99.

14. Fischbach, C.; Chen, R.; Matsumoto, T.; Schmelzle, T.; Brugge, J. S.; Polverini, P. J.; Mooney, D. J. Engineering tumors with 3D scaffolds. Nature methods 2007, 4, 855860 .

15. Fotopoulou, C.; Cho, C.; Kraetschell, R.; Gellermann, J.; Wust, P.; Lichtenegger, W.; Sehouli, J. Regional abdominal hyperthermia combined with systemic chemotherapy for the treatment of patients with ovarian cancer relapse: Results of a pilot study. International Journal of Hyperthermia 2010, 26, 118-126.

16. Friedrich, J.; Seidel, C.; Ebner, R.; Kunz-Schughart, L. A. Spheroid-based drug screen: considerations and practical approach. Nature protocols 2009, 4, 309-324.

17. Friedrich, J.; Eder, W.; Castaneda, J.; Doss, M.; Huber, E.; Ebner, R.; KunzSchughart, L. A. A reliable tool to determine cell viability in complex 3-d culture: the acid phosphatase assay. J. Biomol. Screen. 2007, 12, 925-937.

18. Gerweck, L. E.; Vijayappa, S.; Kozin, S. Tumor pH controls the in vivo efficacy of weak acid and base chemotherapeutics. Mol. Cancer. Ther. 2006, 5, 1275-1279.

19. Goodman, T. T.; Olive, P. L.; Pun, S. H. Increased nanoparticle penetration in collagenase-treated multicellular spheroids. International journal of nanomedicine 2007, 2, 265.

20. Goodman, T. T.; Ng, C. P.; Pun, S. H. 3-D tissue culture systems for the evaluation and optimization of nanoparticle-based drug carriers. Bioconjug. Chem. 2008, 19, 1951-1959.

21. Hirschhaeuser, F.; Menne, H.; Dittfeld, C.; West, J.; Mueller-Klieser, W.; KunzSchughart, L. A. Multicellular tumor spheroids: an underestimated tool is catching up again. J. Biotechnol. 2010, 148, 3-15.

22. Hou, D.; Arimura, M.; Fukuda, M.; Oka, T.; Fujii, M. Expression of cell adhesion molecule and albumin genes in primary culture of rat hepatocytes. Cell Biol. Int. 2001, 25, 239-244.

23. Ingram, M.; Techy, G.; Saroufeem, R.; Yazan, O.; Narayan, K.; Goodwin, T.; Spaulding, G. Three-dimensional growth patterns of various human tumor cell lines 
in simulated microgravity of a NASA bioreactor. In Vitro Cellular \& Developmental Biology-Animal 1997, 33, 459-466.

24. Ivascu, A.; Kubbies, M. Rapid generation of single-tumor spheroids for highthroughput cell function and toxicity analysis. J. Biomol. Screen. 2006, 11, 922-932.

25. Jain, R. K. Transport of molecules, particles, and cells in solid tumors. Annu. Rev. Biomed. Eng. 1999, 1, 241-263.

26. Kelm, J. M.; Timmins, N. E.; Brown, C. J.; Fussenegger, M.; Nielsen, L. K. Method for generation of homogeneous multicellular tumor spheroids applicable to a wide variety of cell types. Biotechnol. Bioeng. 2003, 83, 173-180.

27. Kikuchi, K.; Sumaru, K.; Edahiro, J.; Ooshima, Y.; Sugiura, S.; Takagi, T.; Kanamori, T. Stepwise assembly of micropatterned co-cultures using photoresponsive culture surfaces and its application to hepatic tissue arrays. Biotechnol. Bioeng. 2009, 103, 552-561.

28. Ledoux, S.; Yang, R.; Friedlander, G.; Laouari, D. Glucose depletion enhances Pglycoprotein expression in hepatoma cells: role of endoplasmic reticulum stress response. Cancer Res. 2003, 63, 7284-7290.

29. Lee, J. M.; Mhawech-Fauceglia, P.; Lee, N.; Parsanian, L. C.; Lin, Y. G.; Gayther, S. A.; Lawrenson, K. A three-dimensional microenvironment alters protein expression and chemosensitivity of epithelial ovarian cancer cells in vitro. Laboratory investigation 2013, 93, 528-542.

30. Lei, T.; Fernandez-Fernandez, A.; Manchanda, R.; Huang, Y.; McGoron, A. J. Nearinfrared dye loaded polymeric nanoparticles for cancer imaging and therapy and cellular response after laser-induced heating. Beilstein journal of nanotechnology 2014, 5, 313-322.

31. Lin, R.; Chang, H. Recent advances in three-dimensional multicellular spheroid culture for biomedical research. Biotechnology journal 2008, 3, 1172-1184.

32. Lin, R.; Chou, L.; Chien, C. M.; Chang, H. Dynamic analysis of hepatoma spheroid formation: roles of E-cadherin and $\beta 1$-integrin. Cell Tissue Res. 2006, 324, 411-422.

33. Macchiarini, P.; Chella, A.; Ducci, F.; Rossi, B.; Testi, C.; Bevilacqua, G.; Angeletti, C. A. Neoadjuvant chemotherapy, surgery, and postoperative radiation therapy for invasive thymoma. Cancer 1991, 68, 706-713.

34. Mahoney, B. P.; Raghunand, N.; Baggett, B.; Gillies, R. J. Tumor acidity, ion trapping and chemotherapeutics: I. Acid $\mathrm{pH}$ affects the distribution of chemotherapeutic agents in vitro. Biochem. Pharmacol. 2003, 66, 1207-1218. 
35. Olea, N.; Villalobos, M.; Nunez, M.; Elvira, J.; Almodóvar, J.; Pedraza, V.

Evaluation of the growth rate of MCF-7 breast cancer multicellular spheroids using three mathematical models. Cell Prolif. 1994, 27, 213-223.

36. Olive, P. L.; Durand, R. E. Drug and radiation resistance in spheroids: cell contact and kinetics. Cancer Metastasis Rev. 1994, 13, 121-138.

37. Olivo, M.; Bhuvaneswari, R.; Lucky, S. S.; Dendukuri, N.; Soo-Ping Thong, P. Targeted therapy of cancer using photodynamic therapy in combination with multifaceted anti-tumor modalities. Pharmaceuticals 2010, 3, 1507-1529.

38. Ong, S.; Zhao, Z.; Arooz, T.; Zhao, D.; Zhang, S.; Du, T.; Wasser, M.; van Noort, D.; $\mathrm{Yu}, \mathrm{H}$. Engineering a scaffold-free 3D tumor model for in vitro drug penetration studies. Biomaterials 2010, 31, 1180-1190.

39.Pampaloni, F., Ansari, N., \& Stelzer, E. H. (2013). High-resolution deep imaging of live cellular spheroids with light-sheet-based fluorescence microscopy. Cell and Tissue Research, 352(1), 161-177.

40. Peshwa, M. V.; Wu, F. J.; Sharp, H. L.; Cerra, F. B.; Hu, W. Mechanistics of formation and ultrastructural evaluation of hepatocyte spheroids. In Vitro Cellular \& Developmental Biology-Animal 1996, 32, 197-203.

41. Raghunand, N.; Mahoney, B. P.; Gillies, R. J. Tumor acidity, ion trapping and chemotherapeutics: II. $\mathrm{pH}$-dependent partition coefficients predict importance of ion trapping on pharmacokinetics of weakly basic chemotherapeutic agents. Biochem. Pharmacol. 2003, 66, 1219-1229.

42. Raghunand, N.; He, X.; van Sluis, R.; Mahoney, B.; Baggett, B.; Taylor, C. W.; Paine-Murrieta, G.; Roe, D.; Bhujwalla, Z. M.; Gillies, R. J. Enhancement of chemotherapy by manipulation of tumour pH. Br. J. Cancer 1999, 80, 1005-1011.

43. Rice, G. C.; Hoy, C.; Schimke, R. T. Transient hypoxia enhances the frequency of dihydrofolate reductase gene amplification in Chinese hamster ovary cells. Proc. Natl. Acad. Sci. U. S. A. 1986, 83, 5978-5982.

44. Rice, G. C.; Ling, V.; Schimke, R. T. Frequencies of independent and simultaneous selection of Chinese hamster cells for methotrexate and doxorubicin (adriamycin) resistance. Proc. Natl. Acad. Sci. U. S. A. 1987, 84, 9261-9264.

45. Rodday, B.; Hirschhaeuser, F.; Walenta, S.; Mueller-Klieser, W. Semiautomatic growth analysis of multicellular tumor spheroids. J. Biomol. Screen. 2011, 16, 11191124. 
46. Rowlands, S.; Sewchand, L.; Lovlin, R.; Beck, J.; Enns, E. A Fröhlich interaction of human erythrocytes. Physics Letters A 1981, 82, 436-438.

47. Saxena, V.; Sadoqi, M.; Shao, J. Enhanced photo-stability, thermal-stability and aqueous-stability of indocyanine green in polymeric nanoparticulate systems. Journal of Photochemistry and Photobiology B: Biology 2004, 74, 29-38.

48. Sodek, K. L.; Ringuette, M. J.; Brown, T. J. Compact spheroid formation by ovarian cancer cells is associated with contractile behavior and an invasive phenotype. International journal of cancer 2009, 124, 2060-2070.

49. Sutherland, R. M. Cell and environment interactions in tumor microregions: the multicell spheroid model. Science 1988, 240, 177.

50. Sykes, E. A.; Dai, Q.; Sarsons, C. D.; Chen, J.; Rocheleau, J. V.; Hwang, D. M.; Zheng, G.; Cramb, D. T.; Rinker, K. D.; Chan, W. C. Tailoring nanoparticle designs to target cancer based on tumor pathophysiology. Proc. Natl. Acad. Sci. U. S. A. 2016, 113, E1142-51.

51. Tang, Y. Cancer Therapy Combining Modalities of Hyperthermia and Chemotherapy: in vitro Cellular Response after Rapid Heat Accumulation in the Cancer Cell. 2010. http://digitalcommons.fiu.edu/dissertations/AAI3431329/;

52. Tang, Y.; McGoron, A. J. Combined effects of laser-ICG photothermotherapy and doxorubicin chemotherapy on ovarian cancer cells. Journal of Photochemistry and Photobiology B: Biology 2009, 97, 138-144.

53. Timmins, N. E.; Nielsen, L. K. Generation of multicellular tumor spheroids by the hanging-drop method. Tissue Eng. 2007, 141-151.

54. Tredan, O.; Galmarini, C. M.; Patel, K.; Tannock, I. F. Drug resistance and the solid tumor microenvironment. J. Natl. Cancer Inst. 2007a, 99, 1441-1454.

55. Tredan, O.; Galmarini, C. M.; Patel, K.; Tannock, I. F. Drug resistance and the solid tumor microenvironment. J. Natl. Cancer Inst. 2007b, 99, 1441-1454.

56. van der Zee, J. Heating the patient: a promising approach? Ann. Oncol. 2002, 13, 1173-1184.

57. Walzl, A.; Unger, C.; Kramer, N.; Unterleuthner, D.; Scherzer, M.; Hengstschlager, M.; Schwanzer-Pfeiffer, D.; Dolznig, H. The Resazurin Reduction Assay Can Distinguish Cytotoxic from Cytostatic Compounds in Spheroid Screening Assays. $J$. Biomol. Screen. 2014, 19, 1047-1059. 
58. Wang, S.; Diller, K. R.; Aggarwal, S. J. Kinetics study of endogenous heat shock protein 70 expression. J. Biomech. Eng. 2003, 125, 794-797.

59. Wang, S.; Xie, W.; Rylander, M. N.; Tucker, P. W.; Aggarwal, S.; Diller, K. R. HSP70 kinetics study by continuous observation of HSP-GFP fusion protein expression on a perfusion heating stage. Biotechnol. Bioeng. 2008, 99, 146-154.

60. Wartenberg, M.; Frey, C.; Diedershagen, H.; Ritgen, J.; Hescheler, J.; Sauer, H. Development of an intrinsic P-glycoprotein-mediated doxorubicin resistance in quiescent cell layers of large, multicellular prostate tumor spheroids. International journal of cancer 1998, 75, 855-863.

61. Wartenberg, M.; Gronczynska, S.; Bekhite, M. M.; Saric, T.; Niedermeier, W.; Hescheler, J.; Sauer, H. Regulation of the multidrug resistance transporter Pglycoprotein in multicellular prostate tumor spheroids by hyperthermia and reactive oxygen species. International journal of cancer 2005, 113, 229-240.

62. Weinstein, J.; Eger, R.; Covell, D.; Black, C.; Mulshine, J.; Carrasquillo, J.; Larson, S.; Keenan, A. The Pharmacology of Monoclonal Antibodiesa. Ann. N. Y. Acad. Sci. 1987, 507, 199-210.

63. Wolf, G. T.; Hong, W. K.; Fisher, S. G.; Urba, S.; Endicott, J. W.; Close, L.; Fisher, S. R.; Toohill, R. J.; Karp, D.; Miller, D. M. Induction chemotherapy plus radiation compared with surgery plus radiation in patients with advanced laryngeal cancer. $N$. Engl. J. Med. 1991, 324, 1685-1690.

64. Wong, H. L.; Shen, Z.; Lu, Z.; Wientjes, M. G.; Au, J. L. Paclitaxel tumor-priming enhances siRNA delivery and transfection in 3-dimensional tumor cultures. Molecular pharmaceutics 2011a, 8, 833-840.

65. Wong, C.; Stylianopoulos, T.; Cui, J.; Martin, J.; Chauhan, V. P.; Jiang, W.; Popovic, Z.; Jain, R. K.; Bawendi, M. G.; Fukumura, D. Multistage nanoparticle delivery system for deep penetration into tumor tissue. Proc. Natl. Acad. Sci. U. S. A. 2011b, $108,2426-2431$.

66. Xu, F.; Celli, J.; Rizvi, I.; Moon, S.; Hasan, T.; Demirci, U. A three-dimensional in vitro ovarian cancer coculture model using a high-throughput cell patterning platform. Biotechnology journal 2011, 6, 204-212.

67. Yalowich, J. C. Effects of microtubule inhibitors on etoposide accumulation and DNA damage in human K562 cells in vitro. Cancer Res. 1987, 47, 1010-1015.

68. Yuhas, J. M.; Li, A. P.; Martinez, A. O.; Ladman, A. J. A simplified method for production and growth of multicellular tumor spheroids. Cancer Res. 1977, 37, 3639-3643. 
69. Yun, J.; Tomida, A.; Nagata, K.; Tsuruo, T. Glucose-regulated stresses confer resistance to VP-16 in human cancer cells through a decreased expression of DNA topoisomerase II. Oncol. Res. 1995, 7, 583-590. 


\section{Chapter 2}

2 Background.

2.1 Macromolecule transport in tumor tissues.

Tumors can be broadly classified into two types based on their degree of vascularity, 1) vascular tumors, which have a well-developed blood supply serving all tumor regions and 2) avascular tumors which have poorly developed or no blood supply to them. Nutrients, drugs and other macromolecules are transported via convective flow of blood to a tumor site where they extravasate into tumor interstitium from the discontinuous and wide fenestrations of poorly developed blood capillaries. Upon extravasation, macromolecules depend on convective and diffusive forces to further penetrate into tumor tissue. However, unlike normal tissues, tumor vasculature is often poorly developed and lacks an efficient lymphatic drainage system. This leads to higher interstitial pressure and oncotic pressure in tumors compared to well vascularized tissues. Elevated interstitial pressure impedes extravasation of fluid and macromolecules into the tumor interstitium. Increase in tumor oncotic pressure due to elevated levels of endogenous plasma proteins results in reduced oncotic flow. Hence, in tumors with poorly developed vasculature, diffusion is the dominant mode of transport to deliver macromolecules into the tumor tissue (Jain and Stylianopoulos, 2010; Minchinton and Tannock, 2006; Jain, 1999).

The flux for a diffusing macromolecule is determined by its diffusion coefficient (units: meter $^{2} /$ second) and its concentration gradient in the diffusing medium (in this case the tumor). Macromolecules of large size and high molecular weight have lower diffusion 
coefficients and hence diffuse slower compared to small size molecules ${ }^{6}$. Dreher et al. showed that dextran's of medium molecular weight $(40-70 \mathrm{kDa})$ penetrated more efficiently into tumor tissue compared to dextrans of high size (3 MDa) (Dreher et al., 2006). In tumors, however, the diffusion coefficient is further dependent on available fraction of fluid space (porosity) to diffuse and the binding affinity of macromolecules with tumor cells and extracellular components. Tumors with high packing density (low porosity) of cells offer more resistance to diffusion compared to tumors with low packing density (high porosity). Similarly, molecules with high binding affinity to tumor cells or components of ECM diffuse slowly into the tissue. The charged molecules of ECM, viz collagen (negative charge) and glycosaminoglycan's, hyaluronic acid (positive charge) lead to electrostatic interactions with the macromolecules that can also affect macromolecule diffusion. Interestingly, P-gp mediated drug resistance has also been shown to affect macromolecule diffusion. This was shown by Tunngal et al. using multicellular constructs (MCC), in which diffusion of P-gp substrates (doxorubicin) was enhanced through P-gp positive MCC's, whereas doxorubicin diffusion was hindered when P-gp inhibitors were used to counter MDR (Tunggal et al., 2000).

Limited diffusion of oxygen causes hypoxia and regions with low glucose availability and low $\mathrm{pH}$ due to poor nutrient transport as discussed in detail in chapter 1 . Thus, these regions show altered uptake and corresponding response to respective molecules. Therefore, in order to successfully study and predict the delivery of macromolecules to a tumor, all of the aforementioned factors should be considered.

\footnotetext{
${ }^{6}$ The diffusion coefficient is inversely related to size as per the Stokes Einstein relationship
} 
Although, 2-D cultures provide valuable information regarding macromolecule uptake and function, they cannot satisfactorily recreate the complex conditions of the tumor. The barrier to penetration of macromolecules and the multi-factorial nature of drug resistance conferred by the tumor microenvironment remains a great challenge to the delivery of drugs and ultimately to the efficacy of chemotherapy. Hence, models that reflect the functional and structural complexity of tumors are needed.

\subsection{Mathematical description of transport in tumor spheroids ${ }^{7}$.}

The definite geometrical properties of spheroids (circular and ellipsoidal) and symmetry are advantageous for developing a mathematical model to predict macromolecule transport based on spheroid (porosity, cell uptake) and macromolecule properties (size, charge, surface modifications). Mathematical models are cost efficient in terms of finding combinations of spheroid and macromolecule properties that could result in the optimal distribution of macromolecules by simulating a wide range of cases, instead of conducting independent experiments for each case. This view has led to the formulation of an Advection - Reaction (AR) model for spheroid transport. The first model was reported by Weinstein et al. for studying penetration of antibodies, antibody fragments and free drug moieties (Graff and Wittrup, 2003; Weinstein et al., 1987). Predictions of antibody transport from this model have been validated in experiments from various groups (Thurber and Wittrup, 2008). The basic mathematical framework presented below (Eq (2)) is also applicable to model transport of moieties larger than antibodies, viz nanoparticles.

\footnotetext{
${ }^{7}$ Table describing all model parameters is given in Appendix A.
} 


$$
\frac{\partial \mathrm{C}}{\partial \mathrm{t}}=\frac{1}{\mathrm{r}^{2}} \frac{\partial}{\partial \mathrm{r}}\left[\mathrm{D}_{\mathrm{eff}} \varepsilon \mathrm{r}^{2} \frac{\partial}{\partial \mathrm{r}}\left(\frac{\mathrm{C}}{\varepsilon}\right)\right]-\mathrm{k}_{\mathrm{a}} \mathrm{C}_{\mathrm{bs}} \frac{\mathrm{C}}{\varepsilon}+\mathrm{k}_{\mathrm{d}} \mathrm{C}_{\mathrm{b}}
$$

Equation (2) is the general form of Fick's second law of diffusion in spherical coordinates that relates the rate of change of concentration $\mathrm{C}(\mathrm{M}$; molar concentration) in the spheroid with change of flux as a result of a concentration gradient. This rate is determined by the porosity, effective diffusion coefficient $\mathrm{D}_{\text {eff }}\left(\mathrm{m}^{2} / \mathrm{s}\right)$ and a reaction component governed by association $\left(\mathrm{k}_{\mathrm{a}} ; \mathrm{M}^{-1} \mathrm{~s}^{-1}\right)$ and dissociation $\left(\mathrm{k}_{\mathrm{d}} ; \mathrm{s}^{-1}\right)$ rate constants. Porosity $(\varepsilon)$ is a dimensionless variable that is defined as the fraction of void volume to total volume. For a porous medium (spheroid) immersed in liquid the void volume is filled with fluid and acts as diffusing medium for macromolecules (Fournier, 2011; Truskey et al., 2004).

The ideal diffusion coefficient of a molecule $\left(\mathrm{D}_{0}\right)$ is determined from its size using the Stokes - Einstein relation. However, $\mathrm{D}_{0}$ is not sufficient to describe molecule diffusion in complex media. $\mathrm{D}_{0}$ is modified to the effective diffusion coefficient ( $\left.\mathrm{D}_{\text {eff }}\right)$ that accounts for available volume fraction for diffusion and hydrodynamic interactions between solutes and solid matrix (Eq (3)).

$$
\mathrm{D}_{\mathrm{eff}}=\mathrm{D}_{0} \frac{\mathrm{L}(\lambda)}{\mathrm{F} \tau(\varepsilon)}
$$

In equation (3), $\lambda$ is defined as the ratio of particle radius ( $\left.r_{p}\right)$ to pore radius $(r)$, as $\lambda \rightarrow 0$ solute pore interactions can be ignored. $L(\lambda)$ accounts for the hydrodynamic and steric reduction of diffusion in a pore, for $\lambda<0.4 \mathrm{~L}(\lambda)$ is represented by $(\mathrm{Eq}(5)) . \mathrm{F}$ is the shape factor, $\tau(\varepsilon)$ represents the tortuosity, i.e., increased path length between two points in a 
porous medium that is measured by the distance between the points through connected pores (Eq (5)) (Truskey et al., 2004).

$$
\begin{gathered}
L(\lambda)=(1-\lambda)^{2}\left(1-2.1044 \lambda+2.089 \lambda^{3}-0.984 \lambda^{5}\right) \\
\frac{1}{\tau(\varepsilon)}=1-\frac{2}{3}(1+\varepsilon)(1-\varepsilon)^{\frac{2}{3}}
\end{gathered}
$$

Equations (3), (4), and (5) describe the physical parameters that influence the transport of molecules in a porous medium. Additionally, solutes can bind to cell membranes and also be internalized by the cells. Therefore, the total concentration of solute in a spheroid is the sum of solute in fluid phase $(\mathrm{C})$, solute bound to cell membranes $\left(\mathrm{C}_{\mathrm{b}}\right)$ and solute internalized by the cells $\left(\mathrm{C}_{\mathrm{i}}\right)(\mathrm{Eq}(6))$.

$$
\mathrm{C}_{\text {tot }}=\mathrm{C}+\mathrm{C}_{\mathrm{b}}+\mathrm{C}_{\mathrm{i}}
$$

It is assumed that cells do not migrate and that binding sites on cells are recycled at a constant rate. Hence, concentration of binding sites $\left(\mathrm{Cbs}_{\mathrm{bs}}\right)$ is solely dependent on the association and dissociation of solute on the cell membrane (Eq (7))

$$
\frac{\partial \mathrm{C}_{\mathrm{bs}}}{\partial \mathrm{t}}=-\frac{\partial \mathrm{C}_{\mathrm{b}}}{\partial \mathrm{t}}=\mathrm{k}_{\mathrm{a}} \mathrm{C}_{\mathrm{bs}} \mathrm{C}-\mathrm{k}_{\mathrm{d}} \mathrm{C}_{\mathrm{b}}-\mathrm{k}_{\mathrm{i}} \mathrm{C}_{\mathrm{b}}
$$

The bound solute $\left(\mathrm{C}_{\mathrm{b}}\right)$ is subsequently internalized by cells, exocytosis of solutes (i.e., recycling back to the surface) can be neglected here as it is very slow compared to 
internalization and does not have any significant or direct contribution to the concentration flux. Rate of internationalization is determined by $\mathrm{k}_{\mathrm{i}}$ as shown by Equation (8).

$$
\frac{\partial \mathrm{C}_{\mathrm{i}}}{\partial \mathrm{t}}=\mathrm{k}_{\mathrm{i}} \mathrm{C}_{\mathrm{b}}
$$

Equations (2), (7) and (8) need to be solved simultaneously to obtain total concentration of a solute in the spheroid with the following initial and boundary conditions (I.C, B.C)

I.C: $\mathrm{C}(0, \mathrm{r})=\mathrm{C}_{\mathrm{i}}(0, \mathrm{r})=\mathrm{Cb}_{\mathrm{b}}(0, \mathrm{r})=0 ; \mathrm{Cbs}_{\mathrm{bs}}(0, \mathrm{r})=\frac{2}{\pi} \frac{\mathrm{k}_{\beta} \beta \varepsilon}{\alpha^{2} \mathrm{r}_{\mathrm{p}} \mathrm{N}_{\mathrm{A}}}$

B.C: $\mathrm{C}(\mathrm{t}, \mathrm{R})=\mathrm{C}_{0}$ (Dirichlet Boundary) $; \frac{\partial \mathrm{C}}{\partial \mathrm{r}}(\mathrm{t}, 0)=0$ (Neumann Boundary condition based on spherical symmetry).

The available binding sites $(\mathrm{Cbs})$ for the cells is affected by the porosity $(\varepsilon)$, available binding site fraction ( $\beta$ ) and the surface area of a nanoparticle (a) relative to pore radius $\left(\mathrm{r}_{\mathrm{p}}\right)$ (Goodman et.al.2007). Values of physical parameters such as porosity for different tumor types are available from the literature, it can also be determined from spheroid cross sections using scanning electron microscopy or transmission electron microscopy. Particle radius is determined from scanning electron microscopy or through dynamic light scattering techniques. Determination of the cell uptake rate constants $\left(\mathrm{k}_{\mathrm{a}}, \mathrm{kd}_{\mathrm{d}}\right.$, $\mathrm{k}_{\mathrm{i}}$ ) and binding sites require independent experiments since each cell type has different membrane and uptake properties. 
2.3 Mathematical models of cell uptake kinetics.

The uptake of macromolecules by cells happens via passive diffusion or through energy dependent endocytosis. For macromolecules with size greater than $5 \mathrm{~nm}$, endocytosis is the mode of uptake. Endocytosis happens via absorption of molecules on clathirin or caveolae coated pits on the cell membrane led by membrane invagination, wrapping and subsequent "pinching" of the clathirin/caveolae vesicles into the cell. The uptake of ligands targeted specifically to receptors on cells happens via receptor mediated endocytosis. Two different formulations have been widely used to model molecule uptake into cells; 1) based on adsorption kinetics (Langmuir kinetics) and 2) based on thermodynamics of membrane wrapping (energy formulation).

\subsubsection{Langmuir kinetics}

Langmuir kinetics assumes the cell surface as a Langmuir membrane with finite capacity for binding molecules $\left(\mathrm{N}_{0}\right)$ (Wilhelm et al., 2003). The number/mass of particles adsorbed at any time on the cell surface is proportional to the number of particles in extracellular medium $\left(\mathrm{N}_{\mathrm{ext}}\right)$ and the number of particles that can still bind to the cell surface $\left(\mathrm{N}_{0}-\mathrm{N}(\mathrm{t})\right)$. The extracellular medium acts as a reservoir that is assumed to be a nondepleting source (constant) of molecules at the boundary of the tumor. This is a valid assumption since the number of molecules is very high compared to the number of cells at any time. The number of molecules desorbing from the surface is proportional to the absorbed molecules $(\mathrm{N}(\mathrm{t}))$. The proportionality constants are specified by association $\left(\mathrm{k}_{\mathrm{a}}\right.$; $\left.\mathrm{M}^{-1} \mathrm{~s}^{-1}\right)$ and dissociation $\left(\mathrm{k}_{\mathrm{d}} ; \mathrm{s}^{-1}\right)$ rate constants. Therefore, rate of change number of molecules on cell surface is mathematically expressed by Eq (9) 


$$
\frac{\mathrm{dN}(\mathrm{t})}{\mathrm{dt}}=\mathrm{k}_{\mathrm{a}} \mathrm{N}_{\mathrm{ext}}\left(\mathrm{N}_{0}-\mathrm{N}(\mathrm{t})\right)-\mathrm{k}_{\mathrm{d}} \mathrm{N}(\mathrm{t})
$$

A fraction of adsorbed particles is internalized at a rate $\mathrm{k}_{\mathrm{i}}$ by the cells through endocytosis in equation (10). The internalized particles are also actively recycled back to the cell membrane at an externalization rate, $\mathrm{k}_{\mathrm{rec}}$.

$$
\frac{d N_{i}(t)}{d t}=k_{i} N(t)-k_{r e c} N_{i}(t)
$$

Equations (9) and (10) are solved simultaneously using the following initial conditions $\mathrm{N}(\mathrm{t})$ $=0 ; \mathrm{N}_{\mathrm{i}}(\mathrm{t})=0$ at $\mathrm{t}=0$. The total concentration of solute present in a cell is the sum of $\mathrm{N}(\mathrm{t})$ (mass adsorbed on the cell membrane) and $\mathrm{N}_{\mathrm{i}}(\mathrm{t})$ (mass internalized by the cell).

\subsubsection{Determination of cell uptake constants using Langmuir kinetics.}

The Langmuir kinetic model has been widely used to fit experimental data of cell uptake in order to estimate the rate constants of cell uptake. However, the model cannot be used directly to independently model $\mathrm{N}(\mathrm{t})$ and $\mathrm{Ni}(\mathrm{t})$ since this information is not often readily available from cell experiments. The information available on total cell concentration is the sum of $\mathrm{N}(\mathrm{t})$ and $\mathrm{N}_{\mathrm{i}}(\mathrm{t})$. Therefore, additional experiments that make some a priori assumptions regarding cell uptake or specialized techniques are necessary to explicitly extract $\mathrm{N}(\mathrm{t})$ and $\mathrm{N}_{\mathrm{i}}(\mathrm{t})$. Conventionally, to estimate membrane concentration, cells are incubated with solute of interest at $4^{\circ} \mathrm{C}$ where the internalization and externalization processes are assumed to be dormant (i.e., $\mathrm{ki}_{\mathrm{i}}$ and $\mathrm{krec}_{\mathrm{r}}=0$ ). The measured concentration is fitted to equations (9) and (10) using a non-linear least squares method (example: 
Levenberg-Marquadt) to obtain $\mathrm{k}_{\mathrm{a}}$ and $\mathrm{k}_{\mathrm{d}}$. The uptake experiments are repeated at $37^{\circ} \mathrm{C}$ and the information on $\mathrm{ka}_{\mathrm{a}}$ and $\mathrm{kd}$ obtained at $4^{\circ} \mathrm{C}$ is used to estimate $\mathrm{k}_{\mathrm{i}}$ and $\mathrm{krec}$. It has been mentioned above that association and dissociation rate constants are strongly dependent on temperature and hence using $\mathrm{k}_{\mathrm{a}}$ and $\mathrm{k}_{\mathrm{d}}$ obtained at different temperatures will lead to an inaccurate representation of uptake kinetics. Furthermore, it has been shown that small nanoparticles enter into the cells even at $4^{\circ} \mathrm{C}$ via energy independent pathways (Gottstein et al., 2013).

Different experimental techniques have been reported in the literature to extract explicit information regarding $\mathrm{N}(\mathrm{t})$ and $\mathrm{N}_{\mathrm{i}}(\mathrm{t})$. These are fluorescence quenching and chemical etching of particles adsorbed on the cell membrane. The fluorescence of adsorbed particles is quenched using agents such as trypan blue and the fluorescence of internalized particles is measured to obtain $\mathrm{Ni}(\mathrm{t})$. With improvements in confocal technology, particle tracking and imaging combined with fluorescent cytometry has been used to gather explicit information on $\mathrm{N}(\mathrm{t})$ and $\mathrm{N}_{\mathrm{i}}(\mathrm{t})$. Jin et al. used a single particle tracking method to track carbon nanotube trajectories to calculate endocytosis and exocytosis rates in NIH 3T3 cells (Jin et al., 2009). Gottstein et al. developed a generic method for estimating particle kinetics by mathematically integrating the fluorescence obtained from confocal microscopy with fluorescence cytometry (Gottstein et al., 2013). The experimental technique and an associated mathematical model was used to study the effect of different surface modifications on the uptake of vesosomes, polystyrene and iron oxide nanoparticles in murine macrophages. Cell uptake kinetics are long term events and the studies may extend for 24 hours. This creates the need for live cell culture microscope chambers and other 
specialized equipment for the constant delivery of gas and culture media during imaging. Advanced optics and software for processing the obtained images and particle tracking are also required, which may not be readily available. Additionally, fluorescent measurements of nanoparticles are severely affected by measurement artefacts that arise due to premature leakage of fluorescent agents from the particles. These artefacts may be reduced by covalent conjugation of dye with particles or pre-washing the particles before incubation with cells. However, direct estimation of particle material inside the cell (gold, silver, iron etc.) is preferable when dye leakage is a concern in modeling particle uptake.

Cho et al. have recently shown that gold nanoparticles adsorbed on the surface of a cell can be etched away using a potassium iodide (KI) solution to estimate internalized fraction of the gold nanoparticles (Cho et al., 2009). However, this technique requires the optimization of various parameters, such as incubation time with KI, and concentration of KI required for complete etching of particles adhered to the cell membrane. These parameters are specific for each cell and particle type (Cho et al., 2009). From the above literature studies it is clear that there is a need for a generic, robust technique that can estimate particle uptake parameters from total measured concentration of particles without the need for additional experiments that are specific to cell and particle type. Inverse estimation approach is one such technique that can be used to predict a large number of parameters (based on a physical phenomenon) from experimental data.

\subsection{Inverse estimation}

An inverse problem is defined as the estimation of unknown parameters that appear in a mathematical formulation of a physical system (cell uptake, heat generation etc.) by 
observing the system's response(Costa et al., 2015). If equations (9) and (10) and are treated as a direct, or forward problem, then the values of $\mathrm{k}_{\mathrm{a}}, \mathrm{k}_{\mathrm{d}}$, $\mathrm{ki}_{\mathrm{i}}$ and $\mathrm{krec}_{\mathrm{c}}$ are known and the differential equations can be solved, based on a set of initial conditions, to study the time evolution of cell uptake. Alternatively, an inverse formulation uses the information on $\mathrm{N}_{\text {tot }}(\mathrm{t})$ available from experiments to recover the cell uptake parameters (Orlande et al., 2011; Orlande et al., 2012; Orlande et al., 2014). This set of parameters are denoted by a vector.

$$
\mathrm{P}^{\mathrm{T}} \equiv\left[\mathrm{P}_{1}, \mathrm{P}_{2} \ldots \mathrm{P}_{\mathrm{n}}\right]
$$

In equation (11), $\mathrm{n}$ denotes the number of parameters. The experimental measurement of response or output of a physical system is denoted in vector form by equation (12).

$$
\mathrm{N}_{\text {tot }}^{\mathrm{T}}=\left[\mathrm{N}_{\text {tot }}^{1}, \mathrm{~N}_{\text {tot }}^{2}, \ldots \mathrm{N}_{\text {tot }}^{\mathrm{i}}\right]
$$

where $\mathrm{N}_{\text {tot }}^{\mathrm{i}}=\mathrm{N}_{\text {tot }}\left(\mathrm{t}_{\mathrm{i}}\right), \mathrm{i}=1,2, \ldots . \mathrm{I}$.

The common approach to solve an inverse problem is to estimate the parameter set $\mathrm{P}^{\mathrm{T}}$ with a given set of experimental measurements $\mathrm{N}_{\text {tot }}\left(\mathrm{t}_{\mathrm{i}}\right)$ is by maximizing the likelihood probability density function (pdf) or by minimizing the exponent of likelihood pdf (equation (13)) (Calvetti and Somersalo, 2007; Kaipio and Somersalo, 2004).

$$
\pi\left(\mathrm{N}_{\text {tot }}^{\mathrm{T}} \mid \mathrm{P}^{\mathrm{T}}\right)=(2 \pi)^{-\mathrm{I} / 2}|\mathrm{~W}|^{1 / 2} \exp \left\{-\frac{1}{2}\left[\mathrm{~N}_{\mathrm{Tot}}-\mathrm{V}_{\mathrm{M}}(\mathrm{P})\right]^{\mathrm{T}} \mathrm{W}^{-1}\left[\mathrm{~N}_{\mathrm{Tot}}-\mathrm{V}_{\mathrm{M}}(\mathrm{P})\right]\right\}
$$

$\mathrm{V}_{\mathrm{M}}(\mathrm{P})$ is the set of responses generated by the system (forward problem) for a given set of P. The likelihood pdf specifies the relative probability of different measurement outcomes 
$\mathrm{N}_{\text {tot }}$ with a fixed P. Equation (13) is formulated on the assumption that measurement errors are Gaussian random variables with zero means and covariance matrix W. Furthermore, it is also assumed that the errors in measurements are independent of the parameters $\mathrm{P}$. The likelihood pdf is based on the classic Bayesian Statistics and is not dependent on the modeling of prior information regarding the system and related uncertainty about the unknown parameters. Instead, the probability distribution models for the measurements and unknowns are constructed separately and explicitly.

Bayes theorem specifies the conditional probability of $\mathrm{N}_{\text {tot }}$ occurring given a set of parameters P. It serves as a mechanism to combine new information (experimental measurements) with previously available information (known as the prior). Furthermore, Bayes theorem is a statistical inversion approach based on the following principles, 1) all variables occurring in the inverse problem are considered random; 2) the randomness describes the degree of information related to their realization, which is coded into the likelihood pdf; 3) the solution of the inverse problem is recast in the form of statistical inference from the posterior probability density, which is the solution to the Bayes problem. Bayes theorem is mathematically stated as.

$$
\pi_{\text {posterior }}(\mathrm{P})=\pi\left(\mathrm{P} \mid \mathrm{N}_{\text {tot }}\right)=\frac{\pi(\mathrm{P}) \pi\left(\mathrm{N}_{\text {tot }} \mid \mathrm{P}\right)}{\pi\left(\mathrm{N}_{\text {tot }}\right)}
$$

where, $\pi_{\text {posterior }}(\mathrm{P})$ or $\pi\left(\mathrm{P} \mid \mathrm{N}_{\text {tot }}\right)$ is the posterior distribution of parameters and is interpreted as the conditional probability of $\mathrm{P}$ occurring for a given set of measurements $\mathrm{N}_{\text {tot. }} \pi(\mathrm{P})$ is the prior probability distribution of the parameter set, $\pi\left(\mathrm{N}_{\text {tot }} \mid \mathrm{P}\right)$ is the 
maximum likelihood function and $\pi\left(\mathrm{N}_{\text {tot }}\right)$ is the marginal probability density of measurements.

The solution of Bayes theorem predicts the posterior distributions (Equation (14)) that are sampled using Markov Chain Monte Carlo methods (MCMC). The most widely used algorithm to implement the MCMC method is the Metropolis-Hastings method (Gilks, 2005). The Metropolis-Hastings (MH) algorithm is implemented by choosing a sample distribution $p\left(\mathrm{P}^{*}, \mathrm{P}^{(\mathrm{t}-1)}\right)$ which is used to draw a new candidate sampling $\mathrm{P}^{*}$ given the current state of Markov chain $\mathrm{P}^{(\mathrm{t}-1)}$. Once the proposal distribution is selected, the $\mathrm{MH}$ algorithm is repeated as following:

1) Sample the proposal distribution based on the current state of the Markov Chain.

2) Calculate the acceptance factor

$$
\alpha=\min \left\{1, \frac{\pi\left(\mathrm{P}^{*} \mid \mathrm{N}_{\text {tot }}\right) \mathrm{p}\left(\mathrm{P}^{(\mathrm{t}-1)}, \mathrm{P}^{*}\right)}{\pi\left(\mathrm{P}^{(\mathrm{t}-1)} \mid \mathrm{N}_{\mathrm{tot}}\right) \mathrm{p}\left(\mathrm{P}^{*}, \mathrm{P}^{(\mathrm{t}-1)}\right)}\right\}
$$

3) Generate a random variable $U$ that is uniformly distributed on the interval 0 to 1 .

4) If $\mathrm{U} \leq \alpha$, then set $\mathrm{P}^{(\mathrm{t})}=\mathrm{P}^{*}$, else set $\mathrm{P}^{(\mathrm{t})}=\mathrm{P}^{(\mathrm{t}-1)}$

5) Return to step 1 and repeat until convergence.

The above algorithm generates a sequence of posterior distribution and the inference on this distribution is obtained from the inference of samples $\left\{\mathrm{P}^{(1)}, \mathrm{P}^{(2)}, \ldots . \mathrm{P}^{(\mathrm{i})}\right\}$. However, a certain number of distributions have to be discarded before the Markov Chain reaches equilibrium (known as the burn in period). 
2.5 Silica nanoparticles as model nanoparticles for cell uptake.

Inorganic nanoparticles made from silver, silica and gold offer advantages such as 1) control over size and surface properties, and 2) ease of loading with various fluorescent agents (Zhao et al., 2004). Silica nanoparticles were chosen for our studies since silver and gold nanoparticles flocculate in physiological buffers and are not stable against aggregation. Aggregation increases effective particle diameter and induces high experimental variability that is often difficult to model without explicit information on its dynamics. Moreover, silver particles induce cell toxicity due to leaching of silver ions in physiological buffers. The advantages of silica nanoparticles over other inorganic nanoparticle systems and mechanism of formation is discussed in detail in chapter 3 .

Nanoparticle Preparation: Fluorescent silica nanoparticles were prepared by a slight modification of the reverse microemulsion method (Bagwe et al., 2004). Briefly, $1.6 \mathrm{mg}$ Fluorescein Isothiocyanate (FITC) was reacted with $20 \mu \mathrm{l}$ 3-Aminopropyltriethoxysilane (APTES) in $1 \mathrm{ml}$ ethanol (200 proof) for 6 hours. The ternary emulsion was prepared by adding $7.8 \mathrm{ml}$ cyclohexane, $1.6 \mathrm{ml}$ 1-hexanol (co-surfactant), $1.77 \mathrm{ml}$ Triton X-100 (surfactant), $480 \mu \mathrm{l}$ De-ionized water (18 MOhms), $100 \mu \mathrm{l}$ of APTES-FITC mixture and $100 \mu 1$ TetraEthylorthoSilicate (silica precursor). The mixture was stirred on a rotor plate at 800 r.p.m for 30 minutes after which $65 \mu$ of aqueous ammonia ( $28 \%$ in water) was added. The stirring was allowed to proceed under a nitrogen atmosphere for 48 hours after which the nanoparticles were recovered by adding ethanol to the microemulsion. The recovered particles were washed 2 times with ethanol and 1 time with $\mathrm{NaOH}$ solution $(8 \mathrm{mM})$ to get rid of the unentrapped dye (FITC) and the surfactant. The final yellow 
product (FITC-SiNP) was resuspended in $5 \mathrm{ml}, 8 \mathrm{mM} \mathrm{NaOH}$ solution using sonication and was stored at $4^{\circ} \mathrm{C}$ until further use.

FITC-SiNP were modified with Polyethylene Glycol. Methoxy- PEG- Silane (mPEGSi) and Carboxy-PEG-Silane (cPEGSi) were dissolved in water at $5 \mathrm{mg} / \mathrm{ml}$ and used in for pegylation of the nanoparticle surface. Nanoparticle suspension $(2.5 \mathrm{ml})$ was mixed with $10 \mathrm{ml}$ DI water containing $50 \mu \mathrm{l}$ aqueous ammonia and sonicated for 5 minutes. This was followed by the addition of $15 \mu \mathrm{l}$ of TEOS and $1 \mathrm{ml}$ of PEG solution. The mixture was vigorously stirred at $60^{\circ} \mathrm{C}$ for 15 hours. The particles were washed 3 times using a 100 KDa centrifugation filter to get rid of unattached PEG. Finally, the particles were resuspended in $1 \mathrm{ml} \mathrm{DI}$ water and stored at $4^{\circ} \mathrm{C}$ for further experiments.

Nanoparticle Characterization: The size and surface charge were characterized using dynamic light scattering. Silica content of nanoparticles was estimated using the blue molybdosilicic assay as per manufacturer's instructions. The number of silica nanoparticles was calculated by assuming a monodisperse size distribution and the known density value of silica, i.e., $2.0 \mathrm{~g} / \mathrm{cm}^{3}$. A calibration curve of fluorescent intensity and number of silica particles was prepared by the measurement of fluorescence at $\lambda_{\mathrm{em}}=515 \mathrm{~nm}$ 's and $\lambda_{\mathrm{ex}}=480$ nm's by serial dilutions of FITC- SiNP. The calibration curve was linear within the measured limits of nanoparticle concentration.

2.6 Cell uptake kinetics of nanoparticles.

Skov-3 cells cultured under standard conditions were seeded in a 96 well plate at a density of 25,000 cells/well and allowed to attach overnight in an incubator. PEGylated-FITC loaded silica nanoparticles (PEGSiNp) modified with methoxy-PEG (mPEGSi) or 
carboxy-PEG (cPEGSi) were incubated with McCoy's 5A media for 30 minutes prior to addition to the cells at different concentrations (for determining dose dependent uptake) to determine the optimal concentration at which the cell uptake of nanoparticles saturated. The time kinetics of nanoparticle uptake was studied by incubating the cells with an optimal concentration (determined via dose dependent uptake study to be $100 \mu \mathrm{g} / \mathrm{ml}$ ) of PEGSiNp nanoparticles at different time intervals. Every hour following the incubation (i.e., $1 \mathrm{hr} ., 2 \mathrm{hr} . \ldots . .5 \mathrm{hr}$.), the cells in the wells were washed 3 times with ice-cold DPBS to get rid of free nanoparticles from the extracellular medium. The total nanoparticle concentration in the cells was determined from FITC fluorescence using a multi plate reader. Fluorescence intensity from FITC was corrected by subtracting the intensity obtained from cells without any PEGSiNp-loaded nanoparticles. After the completion of the uptake study, cell number was estimated using the SRB assay. The estimated protein content in each well was converted to cell number using a calibration curve of SRB protein content vs cell number.

The effect of hyperthermia $\left(43^{\circ} \mathrm{C}\right)$ on cell uptake of PEGSiNp loaded silica nanoparticles was studied in a cell culture incubator. The cells were placed in the incubator set at $43^{\circ} \mathrm{C}$ and allowed to equilibrate for 50 minutes, and followed by the addition of the PEGSiNp. The temperature of a well was monitored using a sterile wire thermocouple. Cells were incubated with PEGSiNp at $43^{\circ} \mathrm{C}$ for 1 hour, after which the plates were moved to a $37^{\circ} \mathrm{C}$ incubator. Nanoparticle uptake was determined in a similar manner as described above. FITC fluorescence was converted to the number of Si nanoparticles and divided by number of cells to estimate nanoparticle per cell. 
2.7 Markov Chain Monte Carlo simulations.

Experimental measurements of nanoparticle uptake were imported to MATLAB 13.0® (Math works, MA) and additional data points between each time period were generated using cubic spline interpolation. A total of 1000 data points were generated for the MCMC routine. MCMC simulation was performed with the default number of states as: $\mathrm{N}=1 \mathrm{E} 5$ and chain length $\mathrm{w}=0.01$. The chosen parameter set of uptake rate constants with their respective upper and lower limits is shown in (Table 2.1). It has to be noted that the upper and lower bounds represent a wide range of cell uptake constants. From an initial guess given by the user, equations (9) and (10) were solved using the ode15s solver in MATLAB. The estimated concentration from initial guess was then compared to the experimental data to evaluate the error and an acceptance factor. Based on the acceptance factor, Markov Chain was advanced to choose a new parameter set. Initial iterations of the Markov Chain were discarded (burn in period) and the simulation was run until the Markov Chains of all parameters converged. The robustness of the Monte Carlo method was assessed by choosing various initial guesses for each parameter and from the potential scale reduction factor (PSRF), which is the ratio of the variance of the posterior estimates and in-chain variance. Ideally, PSRF should be close to 1 .

\begin{tabular}{|l|l|l|}
\hline & Lower Bound & Upper Bound \\
\hline $\mathrm{ka}_{\mathrm{a}}\left(\mathrm{M}^{-1} \mathrm{~min}^{-1}\right)$ & $1.00 \mathrm{E}+00$ & $1.00 \mathrm{E}+08$ \\
\hline $\mathrm{k}_{\mathrm{d}}\left(\mathrm{min}^{-1}\right)$ & $2.20 \mathrm{E}-07$ & $2.20 \mathrm{E}-01$ \\
\hline $\mathrm{ke}_{\mathrm{e}}\left(\mathrm{min}^{-1}\right)$ & $2.20 \mathrm{E}-06$ & $2.88 \mathrm{E}-01$ \\
\hline
\end{tabular}




\begin{tabular}{|l|l|l|}
\hline Krec $\left(\min ^{-1}\right)$ & $8.00 \mathrm{E}-06$ & $9.30 \mathrm{E}-02$ \\
\hline
\end{tabular}

Table 2.1 : Lower and upper bounds chosen for different parameter values. The values were chosen from different literature studies and the maximum and minimum values were extended by $10 \%$ to form the bounds. (Cho et al., 2009; Jin et al., 2009; Wilhelm et al., 2003).

Figure 2.1 shows the trace plots of Monte Carlo chains for different parameters, the plots for $\mathrm{k}_{\mathrm{a}}$ and $\mathrm{k}_{\mathrm{d}}$ show a well-mixed ${ }^{8}$ chain with small fluctuations around the mean predicted value of the parameters. The Markov Chain ${ }^{9}$ for $\mathrm{k}_{\mathrm{i}}$ required some burn in, but is well-mixed locally, the predicted values in the burn in period were discarded and only values towards the end were considered. Poor mixing of the chain was observed for the parameter $\mathrm{k}_{\mathrm{rec}}$. However, after a long burn in time, i.e., 4E4 iterations, all chains seemed to converge towards the mean parameter value. The PSRF for $\mathrm{k}_{\mathrm{a}}$ and $\mathrm{k}_{\mathrm{d}}$ was 1 , for $\mathrm{k}_{\mathrm{i}}$ it was 0.87 and $\mathrm{k}_{\mathrm{rec}}$ was 0.61 . The prediction of parameters was also not dependent on initial guess, all parameters converged to within $10 \%$ deviation from the mean parameter value for initial guesses that were an order of magnitude (in some cases 2 to 3 orders of magnitude) apart (Figure 2.2). These results indicate the robustness of the Markov Chain Monte Carlo method in fitting the experimental data.

\footnotetext{
${ }^{8}$ A well-mixed chain is a Markov process near its steady state distribution.

${ }^{9}$ In a Markov process the prediction of next event depends on the previous events. The progression to the steady state is a sequential dependence on adjacent events that is defined as a 'chain'.
} 
Trace plot of association rate ka

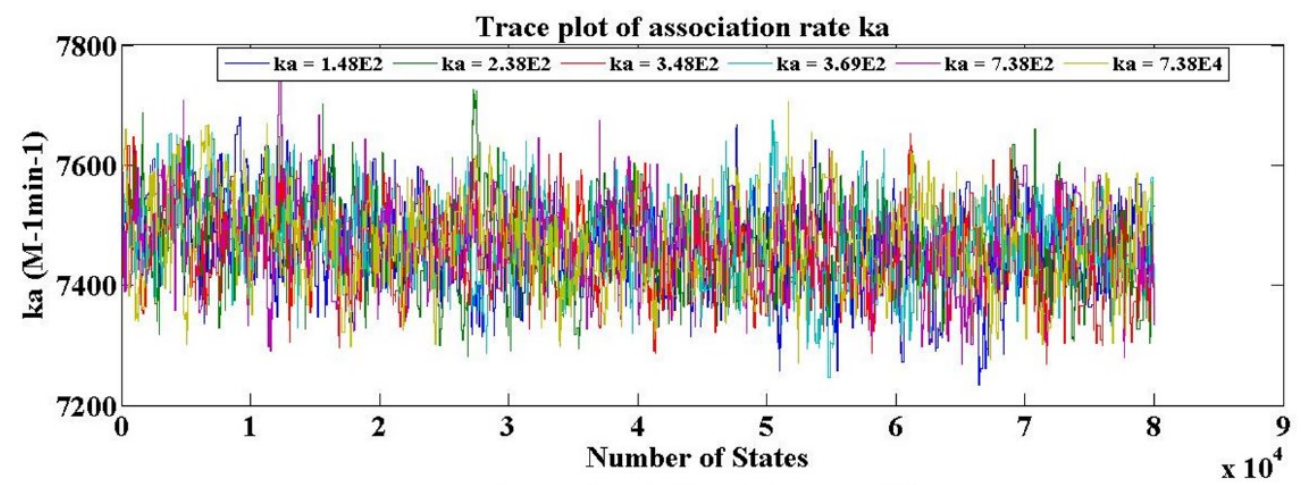

Trace plot of dissociation rate $\mathrm{kd}$
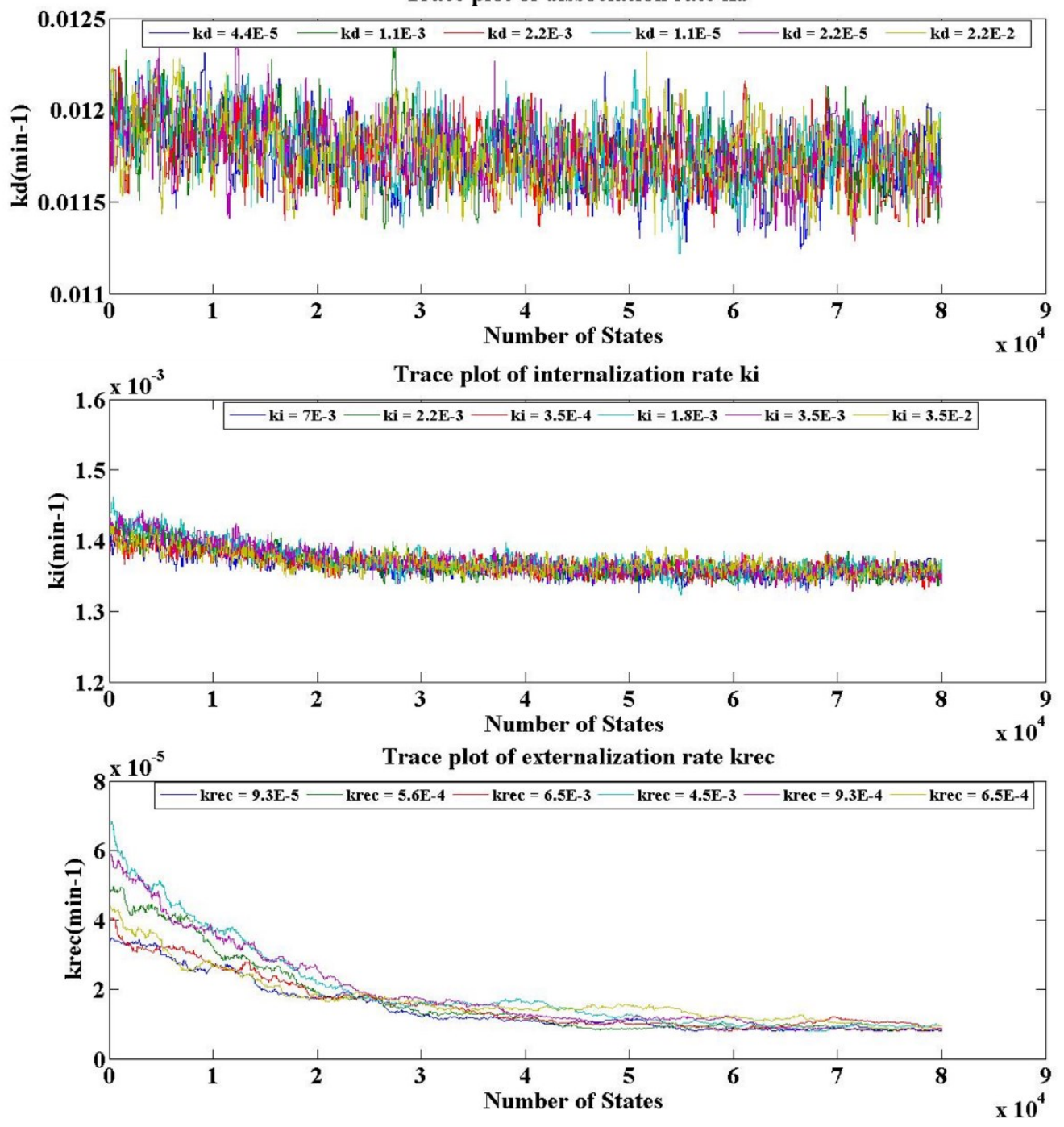

Figure 2.1: Trace plots showing chain mixing of parameters. $k_{a}, k_{d}$ and $k_{i}$, show good mixing while $\mathrm{k}_{\mathrm{rec}}$ shows marginal mixing. 
2.8 Cell uptake experiments and MCMC predictions.

Cell uptake of nanoparticles happens primarily via energy dependent mechanisms such as endocytosis (non-specific, specific/receptor mediated), pinocytosis and macropinocytosis. These processes are dependent on binding of nanoparticles to the cell membrane and subsequent internalization through membrane wrapping and pinch-off into the cell. The interaction of nanoparticles with a cell is primarily determined by the protein corona that forms on the nanoparticle surface due to the adsorption of proteins that are present in incubation media (cell culture and plasma). From the above described events, it is clear that temperature plays a very important role in 1) the physical processes that affect nanoparticles, such as aggregation, 2) protein adsorption on the nanoparticle surface and 3) affecting the cell membrane and/or nanoparticle interaction with the membrane (Mahmoudi et al., 2013b).

The effect of nanoparticle surface functionalization on uptake by Skov-3 cells is shown in (Figure 2.3). The comparison of experimental data and uptake kinetics determined from the parameters predicted by the MCMC method are shown in Figure 2.3. MCMC predictions of cell uptake agree with experimental results. 

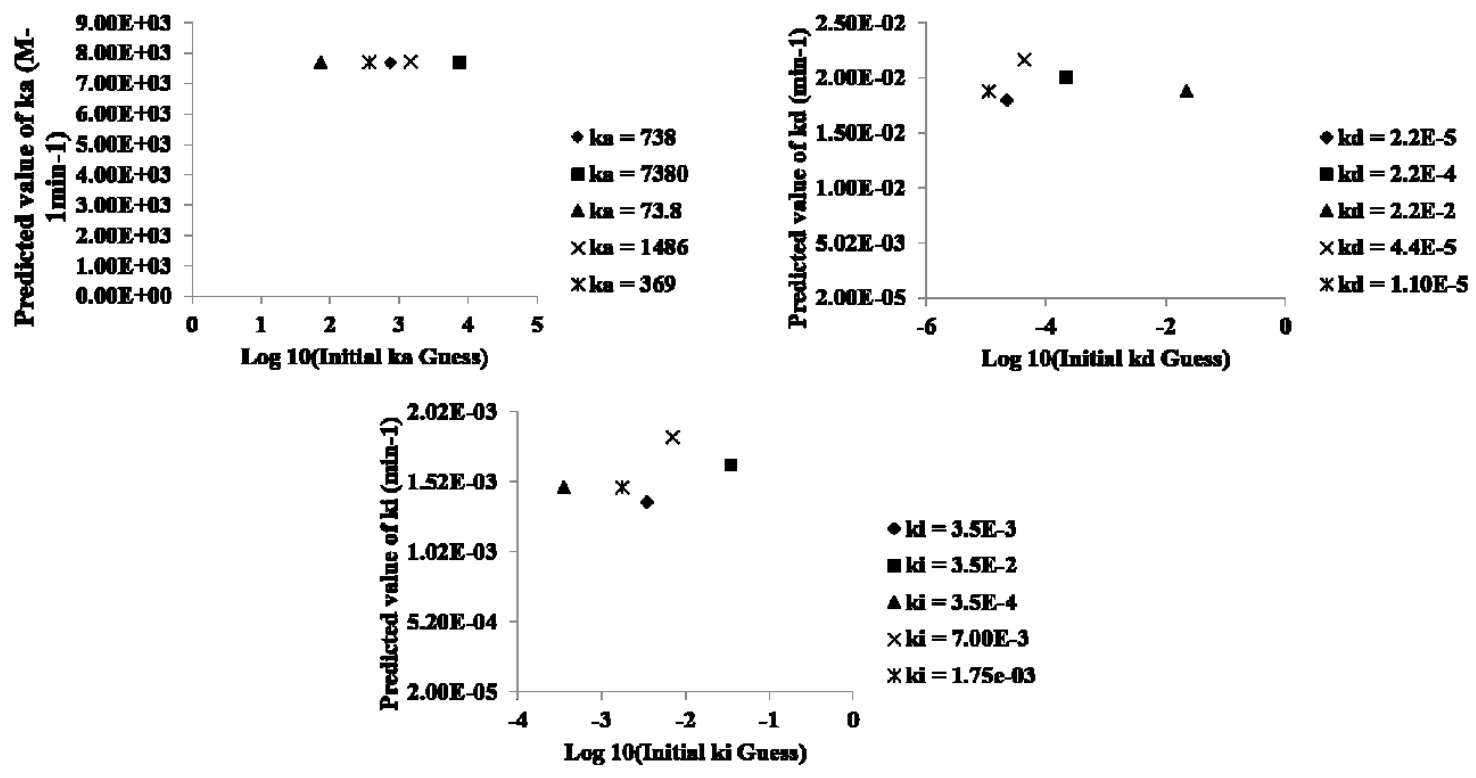

Figure 2.1: Parameter prediction is not dependent on the chosen initial guess. Results show no dependence on initial guess.

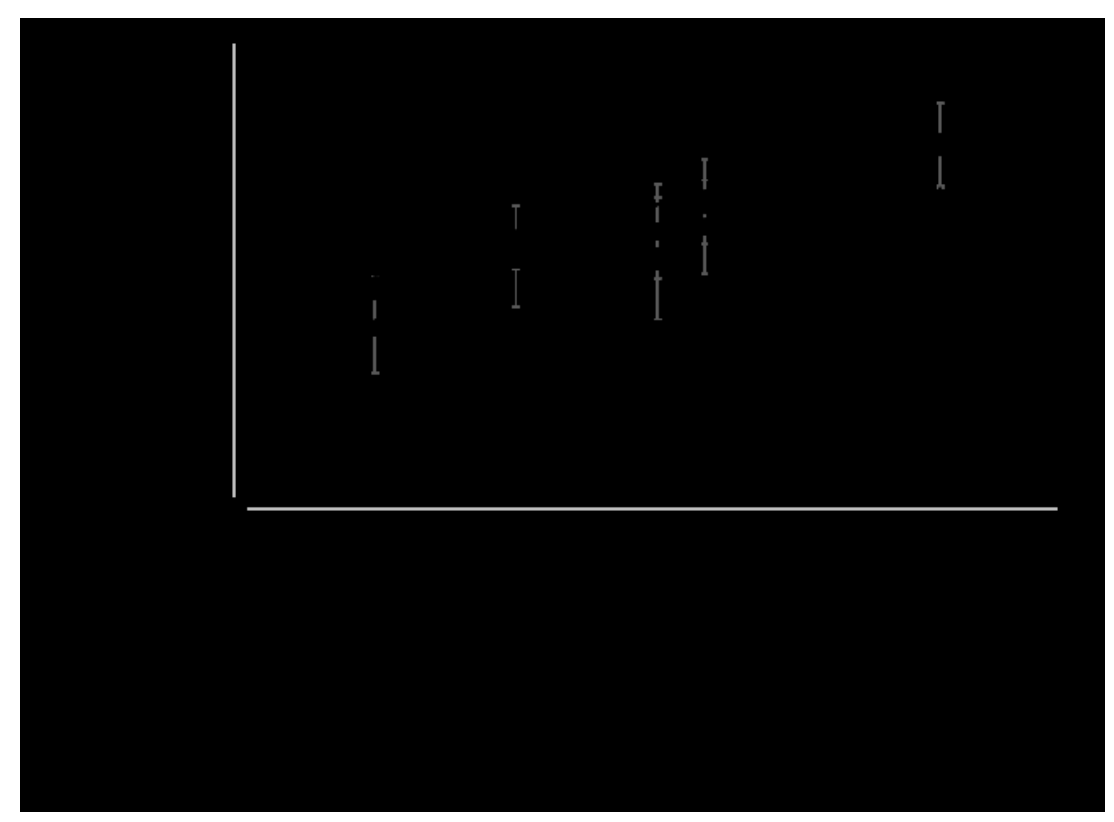

Figure 2.3: MCMC Predictions align closely with experimental measurements for all cases. Image shows predictions of cell uptake overlaid with experimental data. 


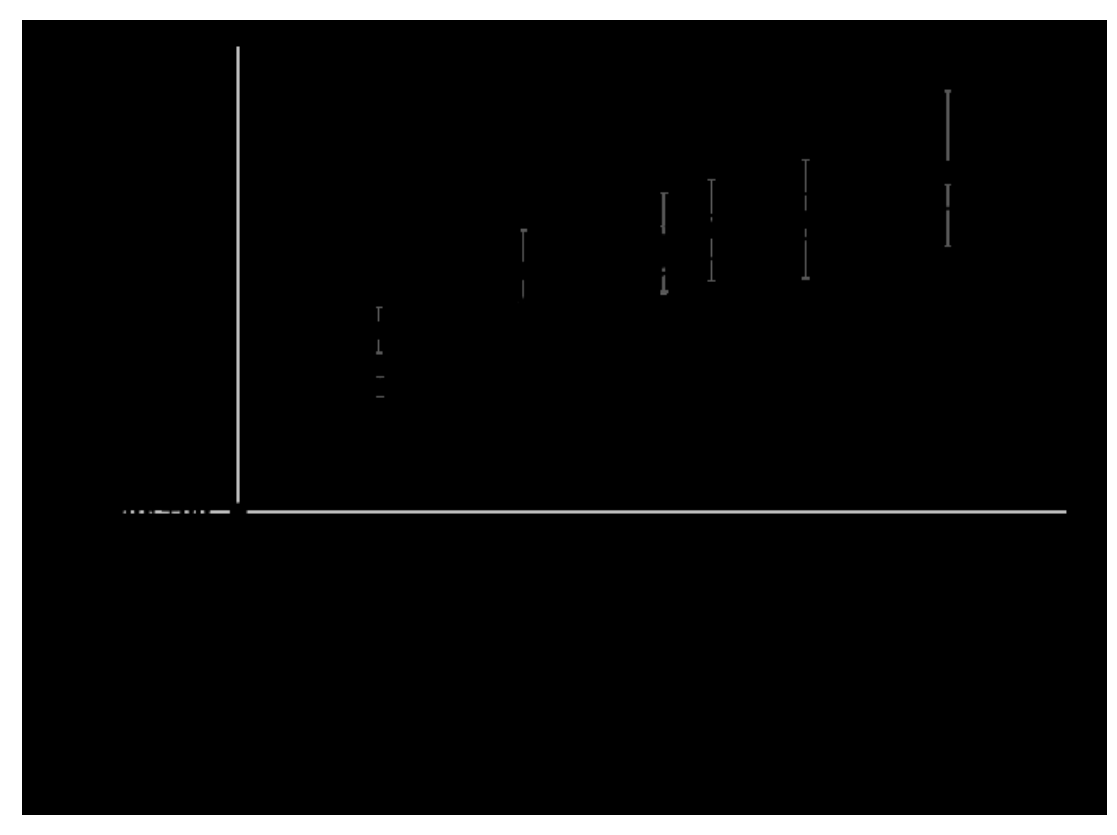

Figure 2.4: MCMC Predictions align closely with experimental measurements for all cases. Image shows predictions of cell uptake overlaid with experimental data.

Silica nanoparticles modified with mPEGSi and cPEGSi had the same size ( $\sim 58 \mathrm{~nm}$ diameter) and surface potential ( -6 mV@ @ pH 7.4). However, mPEGSi particles showed less uptake compared to cPEGSi particles. This is due to the inert methoxy groups that do not interact with components of the incubation media (Gyenge et al., 2011). Carboxy groups have been shown to be more reactive than methoxy groups and hence show more cell uptake than mPEGSi particles (Zhang and Monteiro-Riviere, 2009). mPEGSi particles interact weakly with the cell surface as seen from the predicted value of dissociation constant $\mathrm{K}_{\mathrm{d}}, 5.14 \mathrm{E} 2 \mathrm{M}$ and therefore also have a low internalization rate (Table 2.2). cPEGSi particles show strong binding to the cell surface $\left(\mathrm{K}_{\mathrm{d}} 6.34 \mathrm{E} 5 \mathrm{M}\right)$ and higher internalization rate compared to mPEGSi (Table 2.1). The exposure of cells to hyperthermia at $43^{\circ} \mathrm{C}$ for 1 hour led to faster binding of nanoparticles to cell membrane compared to binding at $37^{\circ} \mathrm{C}$ for both mPEGSi and cPEGSi. This can be seen from the 
initial slope $(0-1 \mathrm{hr})$ of the uptake kinetics curve at $43^{\circ} \mathrm{C}$ which is steeper compared to uptake at $37^{\circ} \mathrm{C}$ (Figure 2.3). With hyperthermia, the predicted association rates for both PEG types were almost twice the values predicted at $37^{\circ} \mathrm{C}$. However, hyperthermia did not increase the number of nanoparticles taken up by cells as no significant difference was found between nanoparticle content at 5 hours for $43^{\circ} \mathrm{C}$ and $37^{\circ} \mathrm{C}$. This is because the cells have a finite capacity for nanoparticle internalization and membrane turnover after internalization. The metabolic processes such as lysosome production have been shown to increase with temperature rise from $4^{\circ} \mathrm{C}$ to $37^{\circ} \mathrm{C}$ to accommodate more nanoparticles into the cell. However, these processes seem to plateau after $37^{\circ} \mathrm{C}$ and a further rise in temperature did not cause a significant increase in lysosome production (Mahmoudi et al., 2013b). Our results are consistent with recent observations by DeWitt et al. which showed that the cell uptake of cisplatin was increased in cells at $42^{\circ} \mathrm{C}$ but no such temperature dependent increase was observed in the uptake of cisplatin conjugated carbon nanotubes (DeWitt et al., 2014). From the experimental measurements and predicted values of internalization $\left(\mathrm{k}_{\mathrm{i}}\right)$ and externalization rate constants $\left(\mathrm{k}_{\mathrm{rec}}\right)$ it can be observed that for cPEGSi particles, kinetic processes at $43^{\circ} \mathrm{C}$ are faster than at $37^{\circ} \mathrm{C}$. Interestingly, for mPEGSi, hyperthermia caused an increase in $\mathrm{k}_{\mathrm{i}}$ whereas $\mathrm{k}_{\text {rec }}$ decreased. 


\begin{tabular}{|c|c|c|c|c|c|c|}
\hline & \multicolumn{3}{|l|}{ mPEGSi@+37º } & \multicolumn{3}{|l|}{ mPEGSi@+43ㄷ } \\
\hline & Predicted Value & $+99 \%$ CI & $-99 \%$ CI & Predicted Value & $+99 \% \mathrm{CI}$ & $-99 \%$ CI \\
\hline $\mathrm{k}_{\mathrm{a}}$ & $9.45 \mathrm{E}+00$ & $9.41 \mathrm{E}+00$ & $9.49 \mathrm{E}+00$ & $1.94 \mathrm{E}+01$ & $1.92 \mathrm{E}+01$ & $1.97 \mathrm{E}+01$ \\
\hline $\mathrm{kd}$ & $1.84 \mathrm{E}-02$ & $1.83 \mathrm{E}-02$ & $1.85 \mathrm{E}-02$ & 5.49E-02 & $5.40 \mathrm{E}-02$ & $5.58 \mathrm{E}-02$ \\
\hline $\mathrm{ki}_{\mathrm{i}}$ & $9.10 \mathrm{E}-05$ & 8.29E-05 & $9.90 \mathrm{E}-05$ & 2.52E-03 & $2.48 \mathrm{E}-03$ & $2.56 \mathrm{E}-03$ \\
\hline Krec & $3.85 \mathrm{E}-03$ & $3.58 \mathrm{E}-03$ & 4.12E-03 & $4.52 \mathrm{E}-05$ & $3.78 \mathrm{E}-05$ & $5.26 \mathrm{E}-05$ \\
\hline & \multicolumn{3}{|l|}{ cPEGSi@+37 $\mathrm{C}$} & \multicolumn{3}{|l|}{ cPEGSi@43든 } \\
\hline & Predicted Value & $+99 \%$ CI & $-99 \%$ CI & Predicted Value & $+99 \% \mathrm{CI}$ & $-99 \%$ CI \\
\hline $\mathrm{k}_{\mathrm{a}}$ & $7.46 \mathrm{E}+03$ & $7.30 \mathrm{E}+03$ & $7.62 \mathrm{E}+03$ & $1.43 \mathrm{E}+04$ & $1.41 \mathrm{E}+04$ & $1.45 \mathrm{E}+04$ \\
\hline kd & $1.18 \mathrm{E}-02$ & 1.14E-02 & $1.21 \mathrm{E}-02$ & 3.03E-02 & 2.95E-02 & $3.10 \mathrm{E}-02$ \\
\hline $\mathrm{k}_{\mathrm{i}}$ & $1.37 \mathrm{E}-03$ & $1.35 \mathrm{E}-03$ & $1.39 \mathrm{E}-03$ & $2.72 \mathrm{E}-03$ & 2.66E-03 & $2.78 \mathrm{E}-03$ \\
\hline $\mathrm{k}_{\text {rec }}$ & $1.94 \mathrm{E}-05$ & 1.81E-05 & 2.07E-05 & $2.15 \mathrm{E}-04$ & 2.02E-04 & $2.27 \mathrm{E}-04$ \\
\hline
\end{tabular}

Table 2.2: MCMC predictions of different uptake parameters for mPEGSi and cPEGSi in Skov3 at $37^{\circ} \mathrm{C}$ and $43^{\circ} \mathrm{C}$. (CI; confidence interval) 
2.9 Simulating nanoparticle transport in spheroids under hyperthermia.

Nanoparticle transport in avascular tumor spheroids depends on particle size, particle uptake by the cells (i.e., $\mathrm{K}_{\mathrm{d}}$ ) and tumor porosity. The effects of hyperthermia on particle properties and cell uptake have been discussed above. Depending on the thermal dose, hyperthermia related thermal damage causes cell death via apoptosis or necrosis (Tang, 2010). Thermal damage to tumor tissue is modeled by well-known Arrhenius

$$
\Omega(r, T)=F \int_{0}^{t \operatorname{texp}} e^{E_{a} / R_{t}}(x, y, t) d t
$$

equation.

In equation (15), $F$ is the frequency or pre-exponential factor (1/s), $\mathrm{E}_{\mathrm{a}}$ is the activation energy barrier $(\mathrm{J} / \mathrm{mole}), R$ is the universal gas constant $(\mathrm{J} /$ Mole- $\mathrm{K})$, and $T_{t}(x, y, t)$ is the absolute tissue temperature at the specified coordinates. The value of $\Omega$ is zero before application of the thermal energy. At $\Omega=1,63 \%$ protein denaturation occurs and at $\Omega=4$ 98\% protein denaturation occurs (Chang and Nguyen, 2004).

Cell death in spheroids as a result of hyperthermia causes an increase in the available fluid fraction (i.e., porosity) for the nanoparticles to access. Therefore, hyperthermia may enhance the transport of nanoparticles into tumor spheroids (Attaluri et al., 2011). Equation (16) describes the change in porosity as a result of cell death under hyperthermia.

$$
\varepsilon_{43}=\varepsilon_{37}+\left(100 \%-\varepsilon_{37}\right) \times(1-\mathrm{S}(\mathrm{r}, \mathrm{T}))
$$


$\mathrm{S}(\mathrm{r}, \mathrm{T})$ is cell survival rate which is related to the Arrhenius parameter $\Omega$ by

$$
S(r, T)=e^{-\Omega / 4}
$$

Modeling nanoparticle transport as a function of porosity change in response to the application of hyperthermia requires solving a system of coupled non-linear partial differential equations. The scheme depicted in (Figure 2.4) illustrates the strategy used in modeling the transport of nanoparticles in tumor spheroids. The system of equations was solved using COMSOL 4.2a. Equations (2), (3), (7) and (8) were solved with corresponding initial and boundary conditions using equations for the transport of diluted species. Equation (15) was solved using the partial differential module with free form scripting (ODE DAE). Free form scripting in COMSOL allows the user to input custom differential equations and their boundary/initial conditions. Since, the equations for thermal damage are not available in COMSOL, ODE DAE had to be used to model the thermal damage using the Arrhenius equation. The temperature profile was obtained from the incubator by placing a thermocouple in a well of a 96 well plate containing media. The discrete temperature values at various time points were imported to COMSOL and a continuous profile was generated by interpolation. The tumor spheroid was modeled as a $2 \mathrm{D}$ circle with another concentric circle of radius $(\mathrm{R}+100 \mu \mathrm{m})$ as the outer domain of the spheroid. A free triangular mesh was used for both domains (i.e., spheroid and outer domain). The mesh of the outer domain was finely resolved (max element size: $10 \mu \mathrm{m}$ and min element size: $1.2 \mu \mathrm{m}$ ) in order to account for concentration discontinuity, or 'jump' at the boundary. The spheroid domain was meshed with max element size equal to $90 \mu \mathrm{m}$. The transport 
simulations for different cases, i.e., cPEGSi at $37^{\circ} \mathrm{C}$ and $43^{\circ} \mathrm{C}$, were simulated for 24 hours. The values of different parameters used in the model are presented in table 2.3.

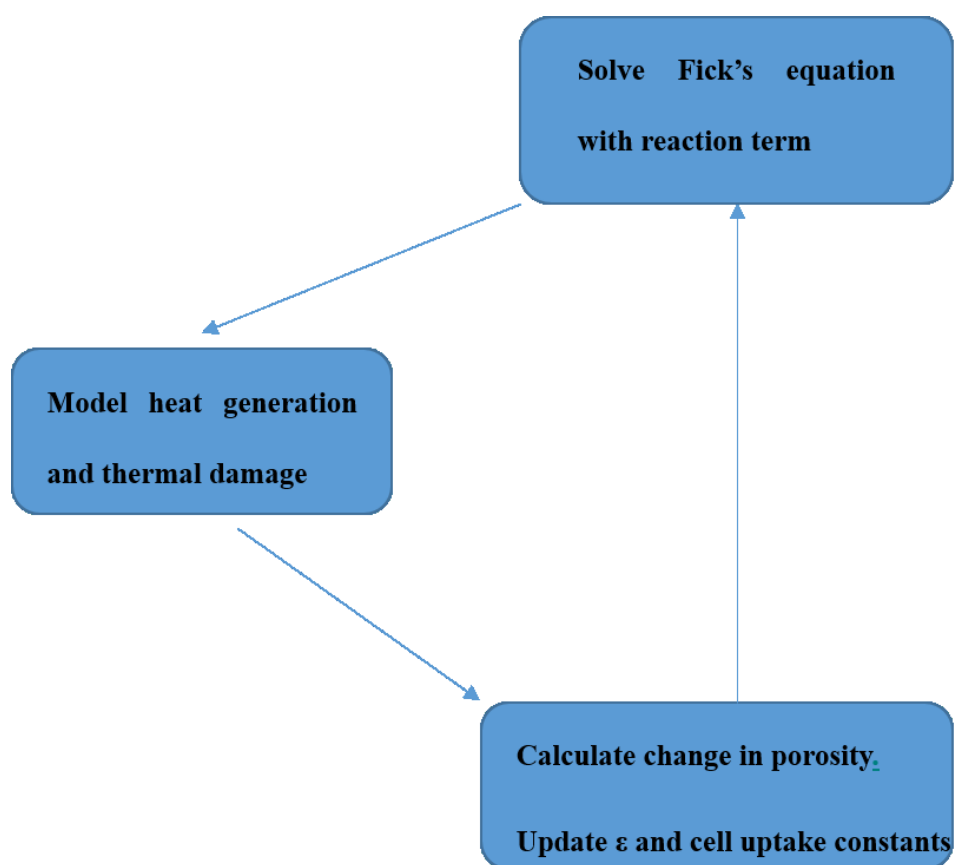

Figure 2.5: Schematic illustrating the Multiphysics strategy used in COMSOL.

\begin{tabular}{|l|l|l|}
\hline Parameter & Value (Unit) & Description \\
\hline $\mathrm{R}$ & $300 \mathrm{E}-6[\mathrm{~m}]$ & Spheroid Radius \\
\hline Bmax & $1.3 \mathrm{E}-7[\mathrm{~mol} / \mathrm{L}]$ & Cell Surface Binding Capacity of \\
CMedium & $1.13 \mathrm{E}-9[\mathrm{~mol} / \mathrm{L}]$ & Spheroids \\
\hline $\mathrm{kB}$ & $1.38 \mathrm{E}-23\left[\left(\mathrm{~m}^{\wedge} 2^{*} \mathrm{~kg}\right) /\left(\mathrm{s}^{\wedge} 2^{*} \mathrm{~K}\right)\right]$ & Boltzmann Constant \\
\hline muCp & $8.94 \mathrm{E}-4\left[\mathrm{~Pa}^{*} \mathrm{~s}\right]$ & Viscosity of Fluid (media) \\
\hline $\mathrm{a}$ & $30[\mathrm{~nm}]$ & Particle Radius \\
\hline
\end{tabular}




\begin{tabular}{|l|l|l|}
\hline $\mathrm{rp}$ & $0.003^{*} \mathrm{R}($ Fournier, 2011) & Collagen Fiber Radius \\
\hline$\lambda$ & $\mathrm{a} / \mathrm{rp}$ & $\begin{array}{l}\text { Ratio of Nanoparticle Radius to } \\
\text { Pore Size }\end{array}$ \\
\hline $\mathrm{Ax}$ & $1.19 \mathrm{E} 38[1 / \mathrm{s}]$ (Attaluri et al., & \\
\hline & $2011)$ & Activation Energy \\
\hline $\mathrm{EAx}$ & $2.57 \mathrm{E} 5[\mathrm{~J} / \mathrm{mol}]$ (Attaluri et al., & Activation Energy Barrier \\
\hline $\mathrm{R}$ & $2011)$ & \\
\hline$\varepsilon$ & $8.31\left[\mathrm{~J} /\left(\mathrm{mol}{ }^{*} \mathrm{~K}\right)\right]$ & Universal Gas Constant \\
\hline
\end{tabular}

Table 2.3: Parameter values used in modeling. Values that could not be measured were adopted from literature studies.

Spheroids were transferred to glass imaging dishes and incubated for 24 hours with cPEGSi at $37^{\circ} \mathrm{C}$ or $43^{\circ} \mathrm{C}$ in the manner similar to monolayer cells. mPEGSi were not tested due to low cell uptake. Prior to imaging, spheroids were treated with (4',6-diamidino-2phenylindole) (DAPI; nuclear stain) for 30 minutes followed by fixation with $4 \%$ formalin. The spheroids were mounted with Fluoroshield and imaged using an Olympus FLV1200 confocal microscope at $10 \mathrm{X}$ objective. For each spheroid, $10 \mu \mathrm{m}$ sections along the $\mathrm{z}$-axis were obtained. Linear attenuation correction and background subtraction of fluorescence intensity were done in FLV1200® software and images were exported for analysis in Image $J$ 1.39. A macro created in Image $J$ was used to locate the center of the spheroid from the DAPI channel and to quantify the fluorescence intensity in the FITC channel. DAPI dye is a small molecule that diffuses rapidly into the spheroid and binds to cell nuclei, hence by using DAPI and FITC the spheroid shape and the distribution of nanoparticle 
fluorescence are easily located. If FITC fluorescence is low or inhomogeneous, especially in deep sections of the spheroid (Figure $2.7 \&$ Figure 2.8) then the spheroid shape is not resolved clearly by the thresholding algorithms. In such cases DAPI dye provides the advantage of locating the spheroid. Firstly, the center and periphery of the spheroid was found by thresholding the images from the DAPI channel. This information was used to quantify fluorescent intensity along a straight line (with length equal to the radius of the spheroid) from the images in the FITC channel. The line was rotated by a $10^{\circ}$ angle and the procedure was repeated. This procedure was adopted to minimize location-related variability within a spheroid (Le Roux et al., 2008). A total of 3 spheroids per each case were analyzed and change in intensity profile with spheroid depth was normalized to intensity at the outer perimeter. Once the intensity curves were obtained, the linear trapezoidal rule was used to calculate the area under the curve (AUC). Another parameter, $\mathrm{W}_{1 / 2}$, half width maximum of concentration was calculated based on the distance in the spheroid at which the total concentration (sum of $\mathrm{Cb}, \mathrm{C}_{\mathrm{i}}$ and $\mathrm{C}$ ) reduced by $50 \%$. A higher AUC value denotes an increase in the amount of nanoparticles in a spheroid whereas a shorter $\mathrm{W}_{1 / 2}$ indicates a deeper penetration (or more shallow concentration decline). The AUC and $\mathrm{W}_{1 / 2}$ from the images were compared to the predictions from the model. Additionally, a fraction of data points from model predictions were measured to calculate the percentage within $95 \% \mathrm{CI}$ of experimental data (Wientjes et al., 2014).

2.10 Simulation and experimental results.

A dynamic diffusion model was used to account for particle aggregation under hyperthermia. cPEGSi particles were incubated in DPBS at $43^{\circ} \mathrm{C}$ and size measurements at various time-points (starting at $1 \mathrm{hr}$. to $24 \mathrm{hrs}$.) were obtained in the Malvern Zetasizer. 
The size was converted to effective diffusion coefficient using equation (3). The effective diffusion coefficient increases initially until 6 hours due to increase in porosity because of cell death (Figure 2.5). After 6 hours, the diffusion coefficient reduces due to increase in particle size due to aggregation. Then, a further decrease in cell viability contributes to an increase in the effective diffusion coefficient (Figure 2.5). The steep reduction in diffusion coefficient at 6 hours is due to the discrete size measurements obtained from the zetasizer. A continuous measurement of size will yield a better representation of the aggregation kinetics.

Spheroid images showed a sharp drop-off of fluorescence at the periphery. This is due to the loss of nanoparticles (and thus FITC) at the periphery during washing and processing steps. To account for this, a window function was introduced in the model (Goodman et al., 2007). It can be seen from Figure 2.6 that after 24 hours' incubation of cPEGSi at $37^{\circ} \mathrm{C}$, particles are localized around the periphery of the spheroid. The moving front of fluorescence drops sharply at a $30 \%$ distance from the periphery and does not progress further towards the center. These findings indicate that diffusion of cPEGSi in Skov-3 spheroids was a slow process (occurring over hours) and was limited to the first few cell layers (Figure 2.8). 

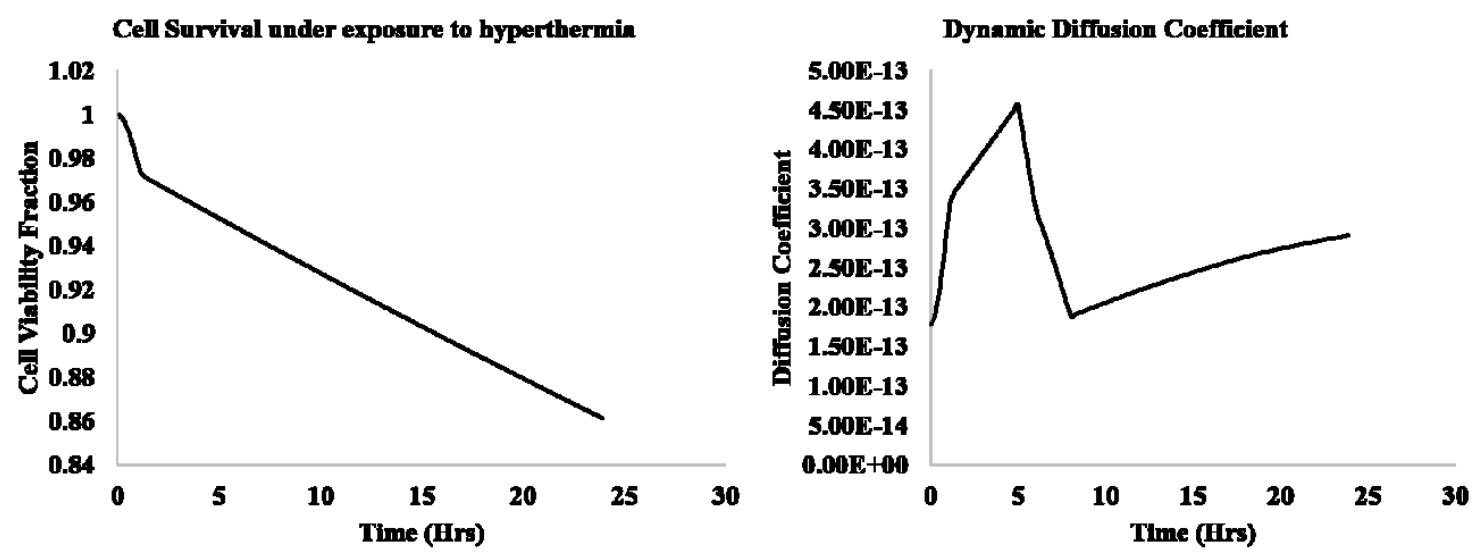

Figure 2.2: Plots showing reduction in cell viability under hyperthermia (left) and changes in effective diffusion coefficient as a result of particle aggregation and change in porosity (right).

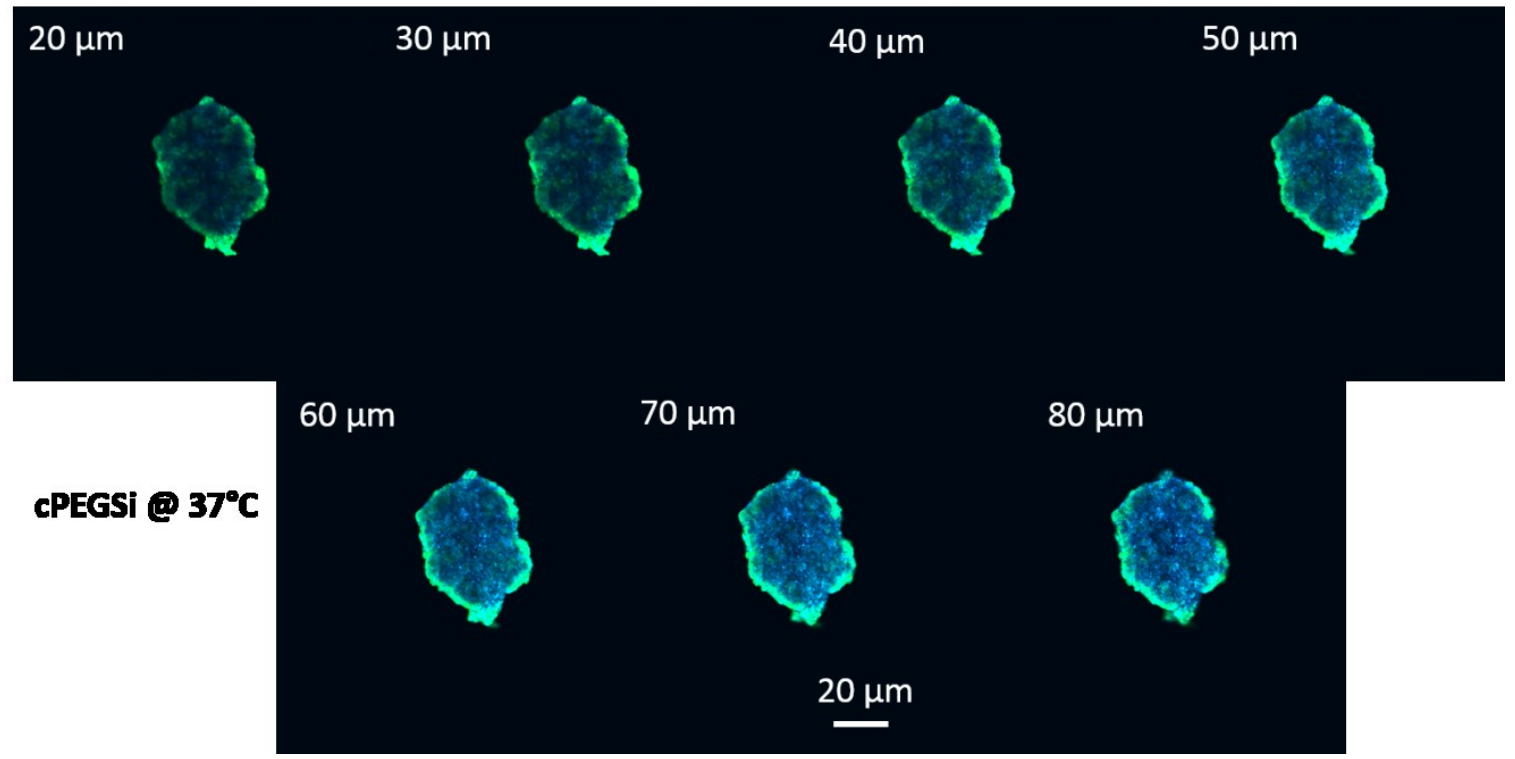

Figure 2.7 : FITC fluorescence is concentrated around the periphery of the spheroid. Confocal sections of Skov-3 spheroids incubated with cPEGSi at $37^{\circ} \mathrm{C}$ with DAPI and FITC channels merged.

Alternatively, spheroids incubated with cPEGSi at $43^{\circ} \mathrm{C}$ for 24 hours showed a less pronounced front and more diffuse fluorescence throughout the spheroid (Figure 2.7). 


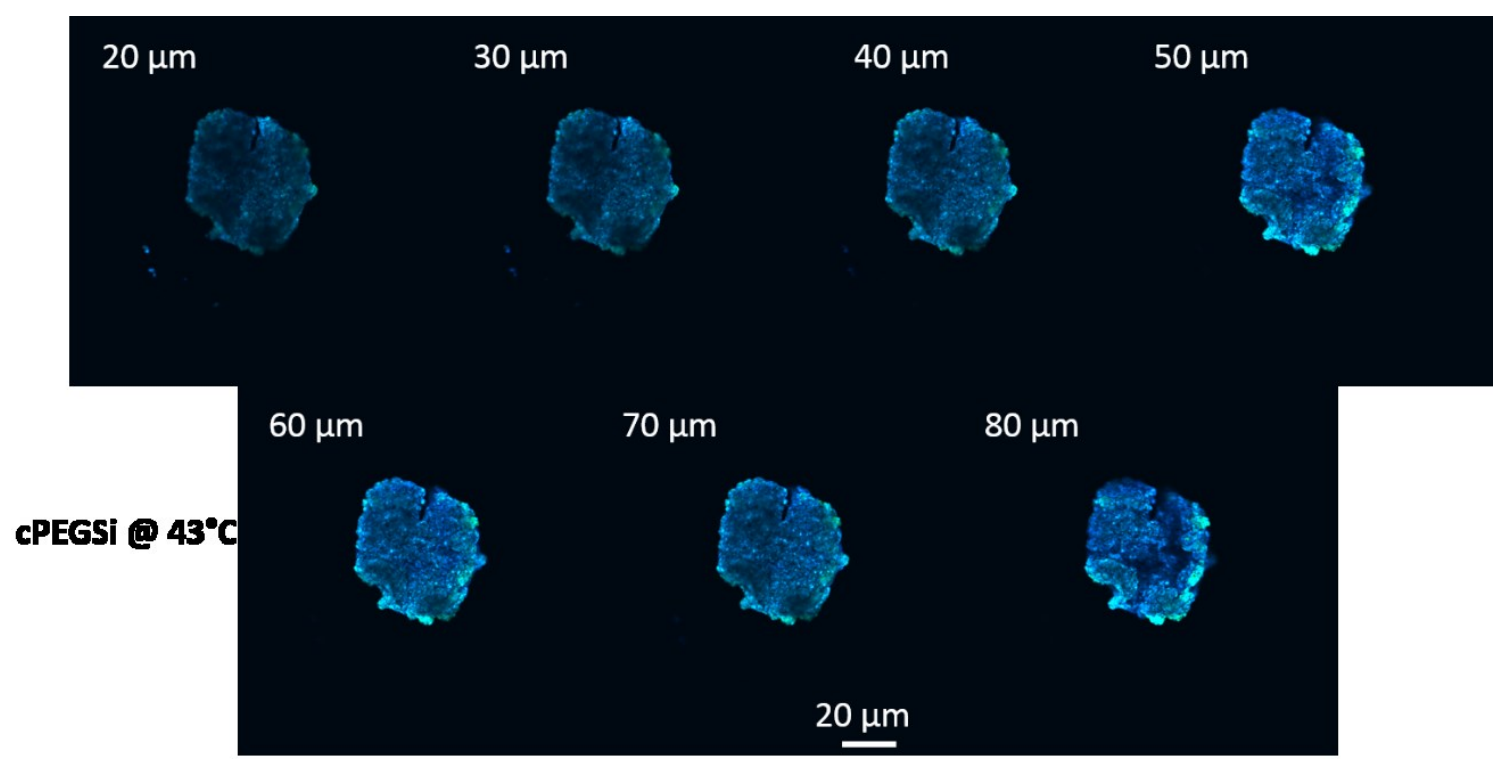

Figure 2.8: FITC fluorescence is more diffuse and is not found around the periphery. Confocal sections of Skov-3 spheroids incubated with cPEGSi at $43^{\circ} \mathrm{C}$ with DAPI and FITC channels merged.

Interestingly, under hyperthermia, MCMC predictions estimated an increase in $\mathrm{K}_{\mathrm{d}}$ which should slow the transport of nanoparticle transport. Concurrently, the internalization rate constant increased at $43^{\circ} \mathrm{C}$, this leads to depletion of nanoparticles in the interstitial space of the spheroid which maintains the concentration gradient and drives the diffusion of nanoparticles. The predicted increase in $\mathrm{K}_{\mathrm{d}}$ may not be sufficient to overcome the diffusive forces. Furthermore, cell death due to thermal damage increased available fluid fraction for the nanoparticles to diffuse (Figure $2.5 \&$ Figure 2.8). The mean AUC and $\mathrm{W}_{1 / 2}$ values calculated from the experiments agree with the values predicted from simulations (Table 2.4). The AUC at $43^{\circ} \mathrm{C}$ was significantly higher than the AUC value at $37^{\circ} \mathrm{C}$. Nanoparticles also penetrated deeper into the spheroid under hyperthermia, $\mathrm{W}_{1 / 2}$ increased to $\sim 50 \%$ at $43^{\circ} \mathrm{C}$ from $\sim 31 \%$ at $37^{\circ} \mathrm{C}$ (Table 2.4 ). However, no significant 
difference was found between the $\mathrm{W}_{1 / 2}$ values for the two cases, owing to variability of the experimental data. A comparison of simulated and experimental data points showed that $55 \%$ and $67 \%$ values of simulated data points fall within the $95 \% \mathrm{CI}$ of experimental data at $37^{\circ} \mathrm{C}$ and $43^{\circ} \mathrm{C}$, respectively (Table 2.4 ).
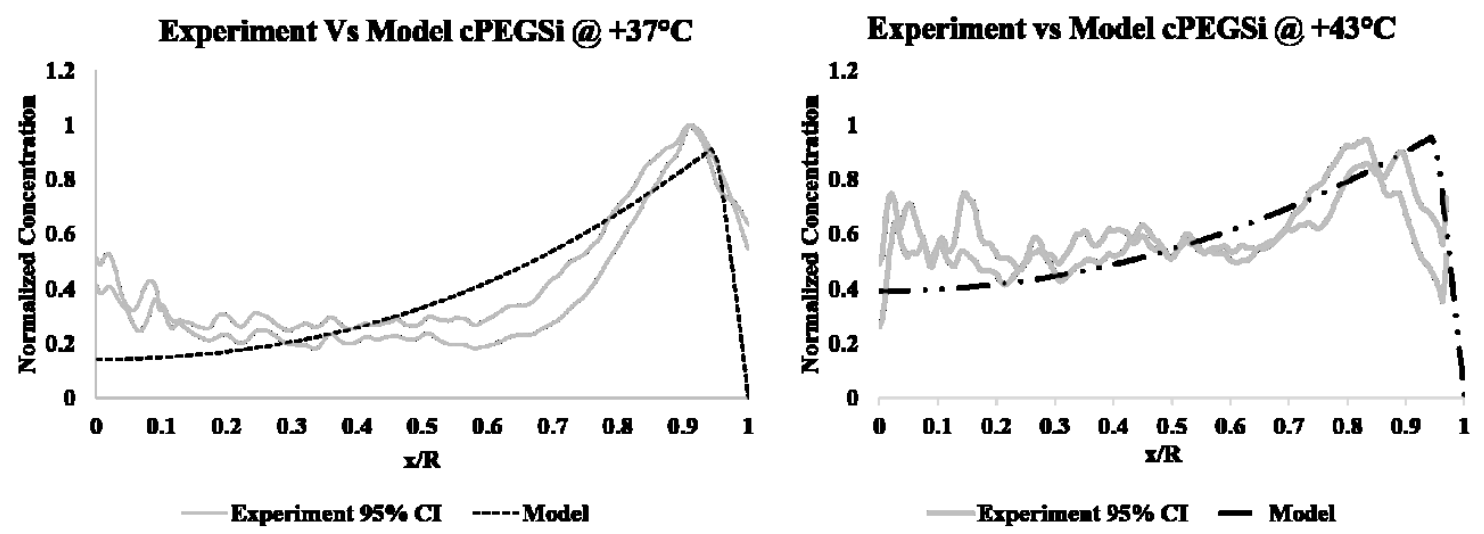

Figure 2.9: Overlay of model predictions with experimental data. The experimental data plotted as two curves denote the higher and lower limits of standard error $(n=3)$.

AUC and $\mathrm{W}_{1 / 2}$ calculations from cPEGSi transport in Skov3 Spheroids.

\begin{tabular}{|l|l|l|l|}
\hline & AUC & $\mathrm{W}_{1 / 2}$ & \multirow{2}{*}{ Fraction 95\% CI } \\
\hline Experiment @ 37 ${ }^{\circ} \mathrm{C}$ & $0.34 \pm 0.06$ & $0.68 \pm 0.15$ & \multirow{2}{*}{$55 \pm 8 \%$} \\
\hline Simulation@ $37^{\circ} \mathrm{C}$ & 0.38 & 0.66 & \\
\hline Experiment @ $43^{\circ} \mathrm{C}$ & $0.61 \pm 0.11^{*}$ & $0.49 \pm 0.12$ & \multirow{2}{*}{$67 \pm 5 \%$} \\
\hline Simulation@ $43^{\circ} \mathrm{C}$ & 0.57 & 0.43 & \\
\hline
\end{tabular}


Table 2.4: Comparison between experimental determination and model prediction of Area under the curve and $\mathrm{W}_{1 / 2}$ values. The fraction indicates percentage of model simulated data points that are within the $95 \%$ confidence limits of experimental data.

The results show that diffusion is the rate limiting step for cPEGSi transport instead of reaction parameters of cell uptake. The moving front progresses into the spheroid as long as free nanoparticles are available at the tumor surface. If the nanoparticle concentration drops to zero at the periphery, i.e., when nanoparticles are removed via washing the spheroids or in-vivo where nanoparticles are removed at the tumor site due to plasma clearance, the front appears to be stuck at a distance from the periphery. In the case of high binding affinity, transport is rate limited by the reaction rate of nanoparticle association. A comparison of gold nanoparticle transport ( $\sim 50 \mathrm{~nm}$ in diameter) with uptake rate constants determined in the literature (Jin et al., 2009) shows that high association, i.e., $\mathrm{ka}_{\mathrm{a}}$ of 7.2E7 $\mathrm{M}^{-1} \mathrm{sec}^{-1}$, leads to high accumulation at the periphery and low penetration into the tumor (Figure 2.9 inset). High accumulation at the periphery results in the 'binding site barrier' effect which further impedes diffusion into the spheroid (Graff and Wittrup, 2003). On the other hand, low affinity nanoparticles lead to deeper penetration of nanoparticles but at an unacceptable cost of low therapeutic efficacy. The tradeoff between penetration and therapeutic efficacy is intrinsic to the nanoparticle system and thus needs to be tailored carefully. 


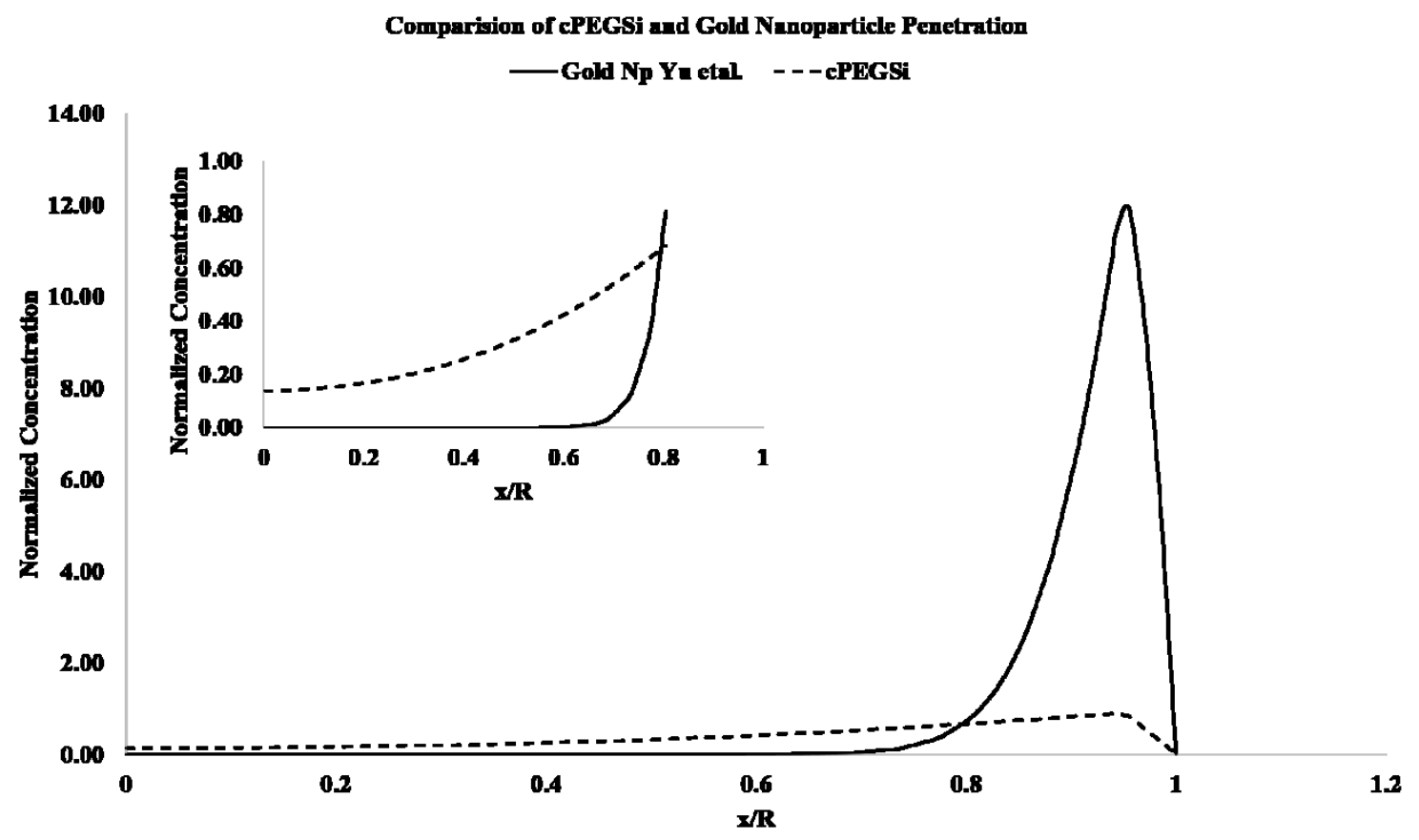

Figure 2.10: COMSOL simulations comparing of transport of $50 \mathrm{~nm}$ gold nanoparticles with high binding affinity to cells and cPEGSi. The uptake parameters for gold nanoparticles were from studies by Jin et al. 2009. The inset shows the particle distribution (zoomed) inside the spheroid.

2.10 Conclusions.

According to current literature, only one other author has reported the mathematical prediction of temperature dependent cell uptake of nanoparticles. The model formulated by Zhang et al. 2011 studied the temperature dependent $\left(6^{\circ} \mathrm{C}\right.$ to $\left.40^{\circ} \mathrm{C}\right)$ uptake of quantum dots in SPCA-1 cells (lung adenocarcinoma) and showed the presence of a temperature independent component of internalization at low temperatures (Zhang et al., 2011). Based on the rate constants obtained by fitting Langmuir kinetics to experimental data, they show that with an increase in temperature from $22^{\circ} \mathrm{C}$ to $37^{\circ} \mathrm{C}$, a reduction in $\mathrm{k}_{\mathrm{a}}$, an increase in $\mathrm{k}_{\mathrm{d}}$ and a plateau in $\mathrm{k}_{\mathrm{i}}$. However, the experimental data by the authors showed a marked increase in uptake at $37^{\circ} \mathrm{C}$. This is counterintuitive as the total content of nanoparticles 
measured in a cell can only increase when there is an increased association of particles with the cell membrane or if there is increased internalization of particles by the cells. The variability in the kinetics of temperature dependent cell uptake of nanoparticles as studied by different authors (DeWitt et al., 2014; Mahmoudi et al., 2013a; Zhang et al., 2011) suggests that this phenomenon may be dependent on the type of nanoparticle and the cell line. The Markov Chain Monte Carlo method used here for predicting cell uptake rate constants provides a unified/general framework for parameter estimation without the need for additional experiments or the need to make specific a priori assumptions regarding cell uptake phenomenon. Since, this approach can predict all rate constants with good confidence and is robust (Figure $2.1 \&$ Figure 2.2), extending it to other nanoparticle systems (liposomes, gold nanoparticles etc.), various cell lines and different conditions can be done easily.

The finite element model developed in this work is an improvement over the existing models of nanoparticle transport. Finite element models for nanoparticle transport in tumor spheroids have been described in the literature for, 1) effect of collagenase on spheroid disruption and subsequent effect on nanoparticle transport (Goodman et al., 2007), 2) dynamic diffusion model of liposomal transport in spheroids (Gao et al., 2013) and 3) macroscopic transport model of heat conduction and magnetic nanoparticle transport in spheroids (Attaluri et al., 2011). The first two models used the conventional method to calculate cell uptake parameters, i.e., perform cell experiments at $4^{\circ} \mathrm{C}$ to estimate $\mathrm{k}_{\mathrm{a}}$ and $\mathrm{k}_{\mathrm{d}}$ and use the information at $37^{\circ} \mathrm{C}$ to estimate $\mathrm{k}_{\mathrm{i}}$. The model by Attaluri et al. only considers nanoparticle transport through the tumor and does not consider uptake by the cells. 
Modeling uptake of nanoparticles by the cells in a spheroid is critical to designing therapeutic interventions for cancer as the drug has to reach the intended target inside the cell to cause an effect. Additionally, the effect of heat on nanoparticle stability and uptake has also not been considered until now. By combining MCMC predictions and finite element simulations, several scenarios can be simulated to find an optimal balance between penetration and cell uptake (MCMC predictions). The transport of drugs to tumors happens through convection which is followed by extravasation of the drug at the tumor site. As mentioned previously, the transport after extravasation is determined by the diffusion into the tumor and the uptake rate of the cells. The uptake rate of nanoparticles is in-turn dependent on the surface and physical properties of the nanoparticle. The combination of parameter estimation using inverse techniques and finite element methods is a suitable approach for this Multiphysics problem. Based on the model predictions, the density/type of surface coating (i.e., number of moieties) and drug loading in nanoparticles can be determined to achieve an efficient therapeutic response. For example, as seen in our experiments, the type of surface coating (i.e. $\mathrm{mPEG}$ vs $\mathrm{cPEG}$ ) plays an important role in determining the uptake. In our case, mPEG particles are not a suitable choice for surface modification due to low cell uptake (Figure 2.3).

The observations from experiments and the corresponding models presented important factors that need to be considered for nanoparticle transport in solid tumors under hyperthermia. These are with regard to effect of hyperthermia on stability of nanoparticle formulation, uptake of nanoparticles by the cell exposed to hyperthermia and the effect of tumor microenvironment on nanoparticle penetration. Colloidal stability i.e., aggregation 
is a kinetic phenomenon dependent on temperature (Figure 2.5). Poor colloidal stability on exposure to hyperthermia may compromise the therapeutic efficacy by further impeding nanoparticle penetration. From a cell uptake perspective, if hyperthermia increases the association rate of particles with the cell, it may overcome the diffusive transport and impede the transport of nanoparticles into the spheroid. Experiments in spheroids showed that in absence of hyperthermia particles mainly localized around the tumor periphery, whereas, exposure to hyperthermia lead to penetration of nanoparticles into the spheroid (Figure 2.7). The advection reaction model showed that the transport of pegylated silica nanoparticles is diffusion dominated (Figure 2.8). The predictions of the model and experiments agreed with each-other (Table 2.4). Spheroids and cells can be cultured in a high throughput format. Therefore, the combination of a mathematical model with high throughput experiments will allow for rapid screening of nanoparticle systems for therapeutic applications.

\subsection{Model limitations.}

The experimental data points vary from model predictions at the center of a spheroid for both $37^{\circ} \mathrm{C}$ and $43^{\circ} \mathrm{C}$ cases. Fluorescence intensity from silica nanoparticles increased near the core of the spheroid (Figure 2.5). This may be due to increased porosity owing to cell necrosis at the center of spheroid. A similar profile of increased fluorescent intensity at the middle of a spheroid due to increased porosity as a result of cell necrosis in the region was reported by Goodman et al. in SiHa cell spheroids (cervical cancer cells; $400 \mu \mathrm{m}$ diameter) (Goodman et al., 2007). The estimation of radially dependent porosity is difficult as reliable techniques for porosity estimation are not available. Electron 
microscopy techniques (SEM and TEM) have been used to image the microstructure of a spheroid and estimate the porosity. However, the processing of spheroids for SEM and TEM, mainly dehydration and critical drying, leads to shrinkage of tissues and alteration to spheroid structure. Furthermore, the alteration in cell uptake of nanoparticles due to aggregation under hyperthermia cannot be estimated inside the spheroid. In monolayer cultures, the uptake was saturated at 5 hours and therefore aggregation which happens after 6 hours did not affect cell uptake. In spheroids, the cell uptake within deep cell layers is delayed due to diffusion. Future models may consider the aforementioned factors for increasing the agreement between experimental data and model predictions.

\section{References}

1. Attaluri, A.; Ma, R.; Qiu, Y.; Li, W.; Zhu, L. Nanoparticle distribution and temperature elevations in prostatic tumours in mice during magnetic nanoparticle hyperthermia. International Journal of Hyperthermia 2011, 27, 491-502.

2. Bagwe, R. P.; Yang, C.; Hilliard, L. R.; Tan, W. Optimization of dye-doped silica nanoparticles prepared using a reverse microemulsion method. Langmuir 2004, 20, 8336-8342.

3. Calvetti, D.; Somersalo, E. An Introduction to Bayesian Scientific Computing: Ten Lectures on Subjective Computing; Springer Science \& Business Media: 2007; Vol. 2.

4. Chang, I. A.; Nguyen, U. D. Thermal modeling of lesion growth with radiofrequency ablation devices. Biomedical engineering online 2004, 3, 1.

5. Cho, E. C.; Xie, J.; Wurm, P. A.; Xia, Y. Understanding the role of surface charges in cellular adsorption versus internalization by selectively removing gold nanoparticles on the cell surface with a I2/KI etchant. Nano letters 2009, 9, 1080-1084.

6. Costa, J. M.; Orlande, H. R.; Velho, H. F. C.; de Pinho, S. T.; Dulikravich, G. S.; Cotta, R. M.; da Cunha Neto, Silvio H Estimation of Tumor Size Evolution Using Particle Filters. Journal of Computational Biology 2015, 22, 649-665. 
7. DeWitt, M. R.; Pekkanen, A. M.; Robertson, J.; Rylander, C. G.; Rylander, M. N. Influence of hyperthermia on efficacy and uptake of carbon nanohorn-cisplatin conjugates. J. Biomech. Eng. 2014, 136, 021003.

8. Dreher, M. R.; Liu, W.; Michelich, C. R.; Dewhirst, M. W.; Yuan, F.; Chilkoti, A. Tumor vascular permeability, accumulation, and penetration of macromolecular drug carriers. J. Natl. Cancer Inst. 2006, 98, 335-344.

9. Fournier, R. L. Basic transport phenomena in biomedical engineering; CRC Press: 2011; .

10. Gao, Y.; Li, M.; Chen, B.; Shen, Z.; Guo, P.; Wientjes, M. G.; Au, J. L. Predictive models of diffusive nanoparticle transport in 3-dimensional tumor cell spheroids. The AAPS journal 2013, 15, 816-831.

11. Gilks, W. R. Markov chain monte carlo; Wiley Online Library: 2005; .

12. Goodman, T. T.; Olive, P. L.; Pun, S. H. Increased nanoparticle penetration in collagenase-treated multicellular spheroids. International journal of nanomedicine 2007, 2, 265.

13. Gottstein, C.; Wu, G.; Wong, B. J.; Zasadzinski, J. A. Precise quantification of nanoparticle internalization. ACS nano 2013, 7, 4933-4945.

14. Graff, C. P.; Wittrup, K. D. Theoretical analysis of antibody targeting of tumor spheroids: importance of dosage for penetration, and affinity for retention. Cancer Res. 2003, 63, 1288-1296.

15. Gyenge, E. B.; Darphin, X.; Wirth, A.; Pieles, U.; Walt, H.; Bredell, M.; Maake, C. Uptake and fate of surface modified silica nanoparticles in head and neck squamous cell carcinoma. Journal of nanobiotechnology 2011, 9, 32.

16. Jain, R. K. Transport of molecules, particles, and cells in solid tumors. Annu. Rev. Biomed. Eng. 1999, 1, 241-263.

17. Jain, R. K.; Stylianopoulos, T. Delivering nanomedicine to solid tumors. Nature reviews clinical oncology 2010, 7, 653-664.

18. Jin, H.; Heller, D. A.; Sharma, R.; Strano, M. S. Size-dependent cellular uptake and expulsion of single-walled carbon nanotubes: single particle tracking and a generic uptake model for nanoparticles. Acs Nano 2009, 3, 149-158.

19. Kaipio, J. P.; Somersalo, E. Computational and statistical methods for inverse problems. Applied mathematical sciences 2004, 160. 
20. Le Roux, L.; Volgin, A.; Maxwell, D.; Ishihara, K.; Gelovani, J.; Schellingerhout, D. Optimizing imaging of three-dimensional multicellular tumor spheroids with fluorescent reporter proteins using confocal microscopy. Molecular imaging 2008, 7.

21. Mahmoudi, M.; Abdelmonem, A. M.; Behzadi, S.; Clement, J. H.; Dutz, S.; Ejtehadi, M. R.; Hartmann, R.; Kantner, K.; Linne, U.; Maffre, P. Temperature: the "ignored" factor at the nanobio interface. ACS nano 2013a, 7, 6555-6562.

22. Mahmoudi, M.; Shokrgozar, M. A.; Behzadi, S. Slight temperature changes affect protein affinity and cellular uptake/toxicity of nanoparticles. Nanoscale 2013b, 5 , 3240-3244.

23. Minchinton, A. I.; Tannock, I. F. Drug penetration in solid tumours. Nature Reviews Cancer 2006, 6, 583-592.

24. Orlande, H. R.; Colaco, M. J.; Dulikravich, G. S.; Vianna, F.; Da Silva, W.; Fonseca, H.; Fudym, O. State estimation problems in heat transfer. International Journal for Uncertainty Quantification 2012, 2.

25. Orlande, H. R.; Dulikravich, G. S.; Neumayer, M.; Watzenig, D.; Colaço, M. J. Accelerated Bayesian inference for the estimation of spatially varying heat flux in a heat conduction problem. Numerical Heat Transfer, Part A: Applications 2014, 65, $1-25$.

26. Orlande, H. R.; Fudym, O.; Maillet, D.; Cotta, R. M. Thermal measurements and inverse techniques; CRC Press: 2011; .

27. Tang, Y. Cancer Therapy Combining Modalities of Hyperthermia and Chemotherapy: in vitro Cellular Response after Rapid Heat Accumulation in the Cancer Cell, 2010.

28. Thurber, G. M.; Wittrup, K. D. Quantitative spatiotemporal analysis of antibody fragment diffusion and endocytic consumption in tumor spheroids. Cancer Res. 2008, $68,3334-3341$.

29. Truskey, G. A.; Yuan, F.; Katz, D. F. Transport phenomena in biological systems. 2004.

30. Tunggal, J. K.; Melo, T.; Ballinger, J. R.; Tannock, I. F. The influence of expression of P-glycoprotein on the penetration of anticancer drugs through multicellular layers. International journal of cancer 2000, 86, 101-107.

31. Weinstein, J.; Eger, R.; Covell, D.; Black, C.; Mulshine, J.; Carrasquillo, J.; Larson, S.; Keenan, A. The Pharmacology of Monoclonal Antibodiesa. Ann. N. Y. Acad. Sci. 1987, 507, 199-210. 
32. Wientjes, M. G.; Yeung, B. Z.; Lu, Z.; Wientjes, M. G.; Au, J. L. Predicting diffusive transport of cationic liposomes in 3-dimensional tumor spheroids. J. Controlled Release 2014, 192, 10-18.

33. Wilhelm, C.; Billotey, C.; Roger, J.; Pons, J.; Bacri, J.; Gazeau, F. Intracellular uptake of anionic superparamagnetic nanoparticles as a function of their surface coating. Biomaterials 2003, 24, 1001-1011.

34. Zhang, A.; Guan, Y.; Xu, L. X. Theoretical study on temperature dependence of cellular uptake of QDs nanoparticles. J. Biomech. Eng. 2011, 133, 124502.

35. Zhang, L. W.; Monteiro-Riviere, N. A. Mechanisms of quantum dot nanoparticle cellular uptake. Toxicological Sciences 2009, 110, 138-155.

36. Zhao, X.; Bagwe, R. P.; Tan, W. Development of Organic-Dye-Doped Silica Nanoparticles in a Reverse Microemulsion. Adv Mater 2004, 16, 173-176. 


\section{Chapter 3}

\subsection{Theranostic nanomedicine for cancer}

The term theranostics is the combination of therapy and diagnostics. Optical imaging in the NIR region has the advantage of low absorption and scattering from the surrounding tissues which enhances the signal to noise ratio from the region of interest where the NIR imaging agent is present. We have investigated the optical, physical and chemical properties of two NIR dyes, viz Indocyanine Green (ICG) and IR820. IR820 is a structural analog of the FDA approved ICG and has more stability against photodegradation as well as improved stability in aqueous media. The combination of IR820 and DOX is a suitable strategy for theranostics and adjuvant hyperthermia owing to the heat generation of IR820 on exposure to NIR laser. However, the free form of DOX and IR820 lacks specificity for tumor specific therapy/imaging and also results in rapid degradation or plasma clearance of the dyes as well as drugs (Fernandez-Fernandez et al., 2012; Saxena et al., 2004). Therefore, a suitable strategy for effective theranostic delivery should simultaneously deliver both agents to the tumor site in sufficient doses without leakage or loss of entrapped moieties. This can be achieved through nanoparticles. The entrapment of multiple agents in a single nanocarrier system presents a challenge to effectively control the release from the nanoparticle of the entrapped agents. Fast or 'burst' release on reconstitution of dried carrier in aqueous media or injection in-vivo leads to loss

of drug from the carrier. The leaked drug will suffer the same fate as its un-encapsulated version, i.e., rapid plasma clearance or in-vivo degradation. This will reduce the available dose at the tumor site. On the other hand, very slow or incomplete release of drug at the 
tumor site is also not desirable as a lethal dose may not be delivered to kill the tumor cells, making the therapy ineffective. For example, DOX and ICG loaded Poly lactic -co glycolic acid (PLGA) nanoparticles synthesized in our laboratory had high initial burst release of DOX and ICG followed by a very slow (50\% in 2 days) and incomplete release of the DOX (Manchanda et al., 2010). For theranostic nanomedicine it is desirable that the imaging agent is retained (i.e., no or very slow release) by the nanoparticle and further that the drug release is triggered on demand. Multiple strategies have been proposed in the literature to tailor the release of entrapped agents. These mainly include research of materials that can respond to various stimuli such as heat, light, sound or conditions of the tumor microenvironment (low $\mathrm{pH}$, hypoxia and matrix metalloproteinases).

The release mechanism based on stimuli can be designed in two ways; (1) via incorporating functional groups that are sensitive to one or more of the above listed stimuli or (2) materials whose structures can be disintegrated (or created) by an external stimulus. External stimuli may cause changes in material structure, dimensions, properties, and lead to their rearrangement or a change in their assembly. In polymers that respond to temperature change, viz, Poly-N-isopropyl acrylamide (PolyNipam), Polyethylene/propylene oxide (PEO/PPO), Pluronic-127, etc., a balance between segmentsegment interactions and segment -solvent interactions can be changed with temperature (Cohn et al., 2009; Motornov et al., 2010). This results in an increase of pore size of the polymer matrix and release of entrapped agents. The polymer properties that define temperature dependent 'phase' change in polymers are known as 'lower critical solution temperature' (LCST) and 'glass transition temperature' $\left(\mathrm{T}_{\mathrm{g}}\right)$. Our work recently illustrated 
a theranostic nano-formulation from a novel polymer, Poly (Glycerol Malate coDodecanedioate) (PGMD) with $\mathrm{T}_{\mathrm{g}}=42^{\circ} \mathrm{C}$. Release of drug (DOX) was enhanced in response to a near infrared dye induced hyperthermia at $43^{\circ} \mathrm{C}$. Increasing the temperature above $T_{g}$ changes the phase of polymer from glassy (no release) to rubbery (release) (Lei et al., 2014). In the case of PolyNipam changing the temperature above $\operatorname{LCST}\left(32^{\circ} \mathrm{C}\right)$ decreases the polymer solvent interactions due to the dominating effect of hydrophobic polymer groups at elevated temperature.

Functional groups such as azobenzene, spirobenzopyran, triphenylmethane, or cinnamonyl are photoactive and can undergo reversible structural changes under UV-vis light. These functional groups change size and shape, or form ionic or zwitterionic species upon irradiation. Absorption of light results in a chemical reaction of the shell of nanoparticle, formation of new functional groups, ionization and the transformation from amphiphilic to hydrophobic. Nanoparticles formulated with the above functional groups are stabilized in the absence of light stimulus. Application of light destabilizes the groups and leads to release of incorporated agents (Li and Keller, 2009; Motornov et al., 2010).

Ultrasonic waves can release entrapped constituents by the generation of heat, which occurs through the absorption of propagating energy by the polymeric membranes. Drug release also occurs by mechanical displacement (referred to as cavitation) of the membrane pores caused by the pressure of ultrasonic waves. Ultrasound mediated therapy has been used with particles such as microbubbles (bubbles composed of gas at the core surrounded by a thin corona of lipid or polymer); the thin corona of the microbubbles is susceptible to oscillations from the ultrasound and can expand and contract in response to 
it. This leads to the creation of strong liquid flows around the membrane which may open the nearby cell membranes. This phenomenon, known as sonophoresis, can lead to enhanced accumulation of drugs when delivered in conjunction with microbubbles or by the controlled release of entrapped constituents in the microbubble (Timko et al., 2010). Similarly, drug release can be modulated with temperature sensitive liposomes; for example, liposomes filled with DOX (Thermodox ${ }^{\circledR}$ ) have been shown to have dose dependent drug release upon exposure to high intensity ultrasound $\left(1300 \mathrm{~W} \mathrm{Cm}^{-2}\right)$ (Dromi et al., 2007).

Materials that respond to conditions of tumor microenvironment such as low $\mathrm{pH}$, tumor enzymes such as matrix metalloproteinases, glutathione and glucose are advantageous to ensure tumor specific drug release. Examples of pH-responsive polymers are weak polyelectrolytes with acidic or basic functional groups (carboxylic, phosphoric, or amino functional groups) (Lee et al., 2008). Functional groups such as hydrazone are $\mathrm{pH}$ labile and can be modified to incorporate chemotherapeutics such as DOX that are released at low pH encountered in the lysosomes of cells (Srinivasan et al., 2016). Gelatin based nanoparticles are degraded by the matrix metalloproteinases (MMP) abundant in tumor tissue and release their constituents (Wong et al., 2011). The design of stimuli responsive theranostic nanoparticles can be quite complex since it requires the assembly of different subunits that should work efficiently as an integrated system. For example, design of the theranostic PGMD formulation required entrapment of chemotherapeutic, near infrared imaging and hyperthermia agent. However, due to the inherent amphiphilicity of the PGMD polymer, the imaging agent leaked rapidly in aqueous solution. Also, the 
physical properties of a system in physiological solutions, viz size, surface charge and colloidal stability should be suitable for deep delivery into the tumor. In some cases, the intrinsic properties of the nanoparticle allow it to be used as an imaging agent and/or an agent for hyperthermia, radiation, or photodynamic therapy. This provides opportunities for image-guided therapy and truly integrated theranostic systems. Some examples of these systems are inorganic nanoparticles formulated from iron and gold.

\subsection{Inorganic nanoparticles.}

The absorption and scattering properties of metallic nanoparticles can be tuned with great control which makes them potential candidates for cancer theranostics. Gold nanoformulations have been used for optical imaging, CT imaging, photo thermal therapy, drug delivery, and combined imaging and therapy (Huang et al., 2008; von Maltzahn et al., 2009). Gold nanoparticles and quantum dots display intense brightness due to strong surface plasmon resonance (SPR), that make them suitable for in-vivo imaging applications. The SPR can be tuned by varying the size, shape and core-shell thickness of the nanoparticles. By varying the size of a gold nanoparticle shell, the absorption can be varied from visible to NIR wavelengths (Jain et al., 2006). In addition to optical properties, gold particles can convert absorbed light into heat in picoseconds and hence have been used for tumor ablation and hyperthermia. Recent studies have shown that hollow gold nanoparticles doped with an excessive amount of DOX release the drug in response to NIR light and low pH triggers (You et al., 2010).

Superparamagnetic iron nanoparticles have been used for Magnetic Resonance Imaging, hyperthermia and magnetic field guided therapy (Wust et al., 2006; Yu et al., 
2008). Superparamagnetic particles (SPM) become mutually attractive under a static magnetic field, hence they have been used to deform hydrogels or ferrogels (the composite of a polymer gel and ferrite is called ferrogel) prompting the release or occlusion of entrapped constituents. When used along-with polymers such as polyvinyl alcohol (PVA) and gelatin, ferrite $\left(\mathrm{Fe}_{3} \mathrm{O}_{4}\right)$ particles conjugated with PVA have been found to inhibit drug release under a static magnetic field that caused magnetic particles to align and seal the pores of the ferrogel (Tai et al., 2009).

Semiconductor metals such as cadmium, selenium and zinc have been used to create ultra-small (6.0 - $15 \mathrm{~nm}$ 's) nanoparticles (quantum dots) that display high luminescence, quantum yield and tunable excitation and emission wavelengths. Due to these advantages, quantum dots have been extensively used for cancer imaging applications (Gao et al., 2004; Yi et al., 2014). For theranostic applications they have been used as cores with a gold, silver or silica shell. Due to their size they are effectively retained by the nanoparticle since the pores within the nanoparticles are too small for the QD's to pass through. Rare earth metals from lanthanides, yittribium and europium have also been used recently for cancer imaging and theranostics. Lanthanide nanoparticles can up-convert NIR light to UV light and when used in conjunction with UV labile bonds can trigger agent release on NIR light exposure (Chatterjee and Yong, 2008). The up-conversion from NIR to UV light is not desirable for in-vivo applications due to the damage caused by UV radiation to the healthy tissues adjacent to the tumor. Apart from gold and iron, inorganic nanoparticles suffer from a lack of biocompatibility associated toxicity. Gold nanoparticles suffer from a lack of stability against aggregation in physiological solutions and large size 
$(>150 \mathrm{~nm})$ is needed for NIR absorbance. Iron nanoparticles require expensive MRI equipment to be used for theranostics.

Silica nanoparticles (amorphous, mesoporous and organically modified) possess the desired properties of an effective theranostic probe, i.e., low toxicity, ease of preparation and modification, good control over size and scale up potential. Mesoporous silica nanoparticles have been extensively used for triggered drug release by 'gating the pores' with stimuli responsive materials (pH and heat) (Slowing et al., 2008). For imaging, silica nanoparticles have been combined with other nanoparticles (iron and gold). Silica particles are considered as "Generally Recognized as Safe" (GRAS) by the FDA, although current research is being undertaken to evaluate and exclude any risk factors associated with their nano-dimensions. Reports by Park et al. have shown luminescent porous silicon particles can self-destruct and be excreted via renal clearance in mice (Park et al., 2009). Recently, fluorescent silica nanoparticles developed at Cornell University, known as "CDots" have been approved for human clinical trials to image human myeloma (Friedman, 2011). However, Silica nanoparticles, especially organically modified silica nanoparticles, have not been studied for image guided cancer therapy.

\subsection{Silica nanoparticles.}

Silica particle synthesis was first reported by Stober and colleagues in 1968 through the base catalyzed condensation of tetraethyl orthosilicate (TEOS) in the presence of aqueous ammonia $\left(\mathrm{NH}_{3}\right)$ (Stöber et al., 1968). The mechanism of particle formation is through the coalescence of condensed TEOS nuclei in a process known as "seed growth". Random Brownian motions in solution bring the nuclei closer to each other resulting in the 
growth of particles. The size of the particles can be varied by varying ratio of TEOS to $\mathrm{NH}_{3}$, reaction time and stirring speed. The disadvantages of the Stober synthesis are high particle polydispersity (wide distribution of particle sizes) and poor entrapment of agents that are not soluble in water or ethanol.

Silica nanoparticles have also been synthesized using the reverse microemulsion method (RME). RME is a Water in Oil (W/O) emulsion system with surfactants such as Triton-X100, Dioctyl sulfosuccinate (AOT), Polyoxyethylene (5)- nonylphenylether (IGEPAL-CO520), sodium dodecyl sulfate (SDS), etc. (Bagwe et al., 2004). The oil phase is comprised of cyclohexane, hexanes, heptane, pentane, etc. In the presence of water, at critical micellar concentrations (CMC), the surfactant molecules form reverse micelles that serve as "nanoreactors" for particle growth and dye entrapment. The reaction mechanism is similar to the Stober synthesis, i.e., base catalyzed condensation of TEOS in the presence of $\mathrm{NH}_{3}$. Brownian collisions cause nuclei transfer between micelles resulting in particle growth, which happens until collision forces can overcome the interfacial tension of the micelles (Arriagada and Osseo-Asare, 1999). Additionally, nanoreactors bring the molecules to be entrapped in close proximity to the silica matrix increasing their interaction with the particles. Particles with small size $(50 \mathrm{~nm})$ and narrow distributions can be obtained through this method by varying surfactant to water ratio (R) and ratios of TEOS or $\mathrm{NH}_{3}$. Other silica precursors have been used in place of TEOS as well as other catalysts, i.e., HCL (acid catalysis) and amino acids (1-lysine, 1-arginine) to formulate silica nanoparticles (Hartlen et al., 2008). 
Ormosil nanoparticles were pioneered by Prasad et al. using a ternary microemulsion system comprised of an oil phase, surfactant, co-surfactant and aqueous phase (Roy et al., 2003). The precursor molecule is an organic silane monomer, viz Triethoxyvinylsilane (VTES). The parameters of the microemulsion can be varied to achieve particles in size ranging from $25 \mathrm{~nm}$ to $80 \mathrm{~nm}$. Functionalization with amine groups offers the flexibility of further modifying the particle surface for attaching tumor targeting moieties (anti-HER2, folic acid, etc.) or with stealth polymers such as Polyethylene Glycol to evade capture by the macrophages of the reticuloendothelial system. Ormosil Np's have been used for chemotherapeutic, photodynamic, gene delivery and bio imaging applications (Kumar et al., 2010; Nakamura, 2012; Shan, 2004). Multifunctional Ormosil Np's entrapped with iron oxide particles and a fluorophore have been reported for MRI and optical imaging applications (Kumar and Roy, 2014; Law et al., 2008).

\subsection{Materials and Methods}

\subsubsection{DOX IR820 Loaded Ormosil Synthesis}

The NIR dye IR820 was entrapped inside the Ormosil matrix whereas DOX was loaded via physical adsorption (called FDSIR820) or via a covalent linkage (called CDSIR820) on the surface of the Ormosil Np's. A silica shell was grown over the DOX layer and was functionalized with amine (-NH2) groups (Figure 3.1). The covalent linkage of DOX to Ormosil was done via a DOX silane conjugate. Five milligrams DOX-HCl was reacted with 2 mole excess Triethylamine (TEA) in $500 \mu$ anhydrous DMSO. This solution was vigorously stirred for 6 hours at room temperature in the dark followed by the addition of $20 \mu \mathrm{l} 3$-(Triethoxysilyl) propylisocyanate (ICPTES). The reaction was allowed to 
proceed for 18 hours and the product (DOX-ICPTES) was used without further purification. Organically modified silica nanoparticles (Ormosil) were prepared by a modification of the ternary microemulsion method developed by Prasad and colleagues (Roy et al., 2003). Briefly, $0.44 \mathrm{mg}$ of Dioctyl sulfosuccinate sodium salt (AOT) was dissolved in $20 \mathrm{ml}$ of deionized water followed by the addition of $800 \mu \mathrm{l} 1$-butanol resulting in a clear solution. To this, $800 \mu$ of DMSO containing IR820 (25 mM) was added. Void nanoparticles were created by adding DMSO without the dye. After this, $125 \mu$ of the silica precursor Triethoxysilane (VTES) was added and stirred for 30 minutes. Ormosil nanoparticles were precipitated by the addition of $20 \mu \mathrm{l}$ aqueous ammonium hydroxide (NH4OH; $28 \%$ in water) and stirring for 18 hours at room temperature.

For DOX loading, $500 \mu \mathrm{l}$ of free DOX in DMSO or $500 \mu \mathrm{l}$ DOX-ICPTES in DMSO was added along with $75 \mu$ VTES to the precipitated particles and stirred for 6 hours. An additional shell of silica was grown by adding $100 \mu$ l of VTES. Amine groups were grafted on the surface by the addition of $10 \mu 13$ - Aminopropyltriethoxysilane (APTES) 30 minutes after the addition of VTES. Finally, after 18 hours of additional stirring the obtained particles were dialyzed for 50 hours using a 12-14 KDa cellulose membrane to remove the free dyes/drugs (IR820, DOX), the surfactant AOT and the co-surfactant 1-butanol. The dialyzed particles were refrigerated until further use. Blank Ormosil nanoparticles without any dye and drug are referred to as $\mathrm{Si}$, with unconjugated DOX are referred to as FDSIR820 and nanoparticles with DOX-ICPTES conjugate are denoted as CDSIR820. 


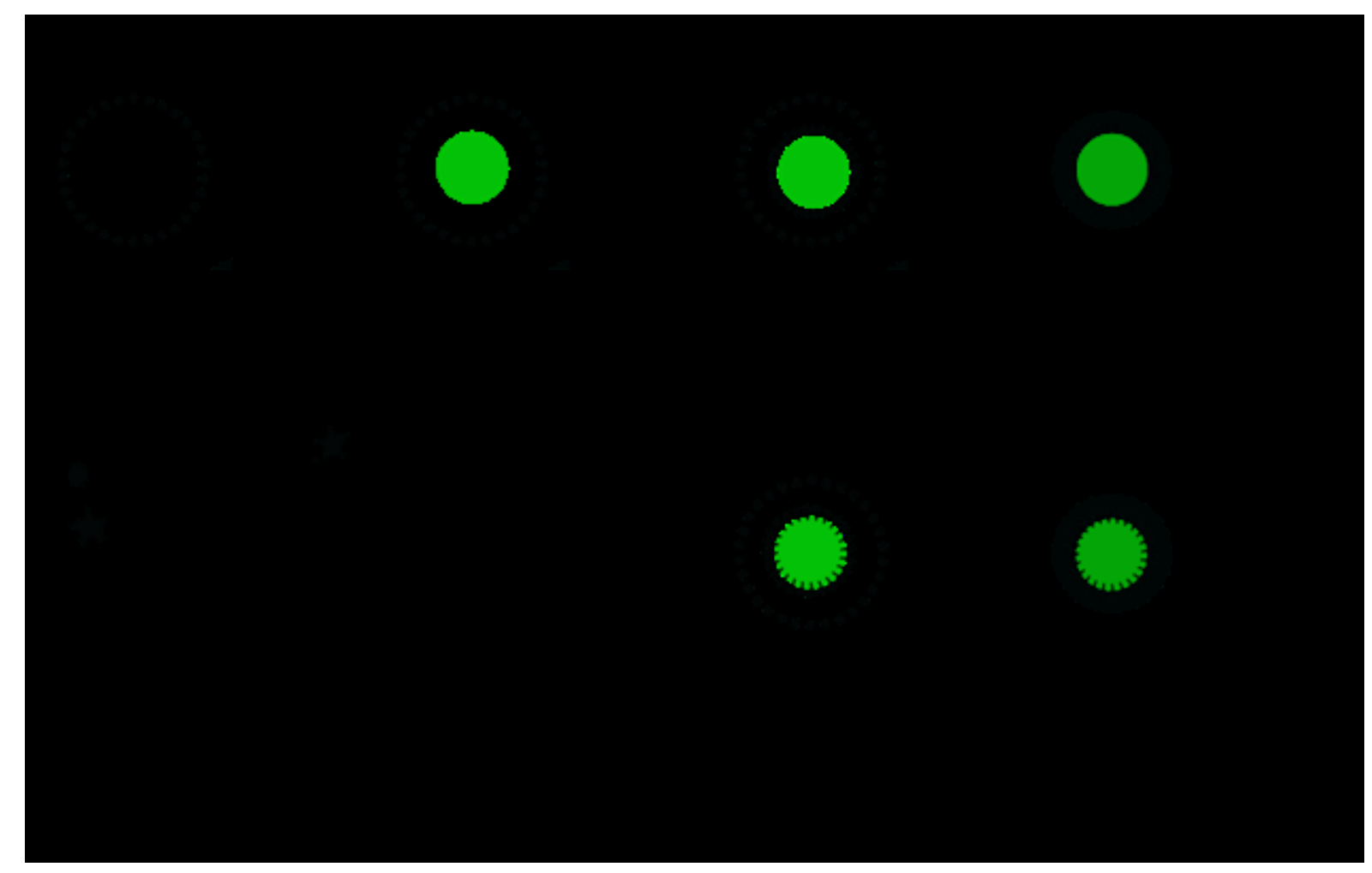

Figure 3.1: Schematic of nanoparticle formation in microemulsion and different modes of DOX loading.

The surface of Ormosil nanoparticles was modified by the addition of Succinimidyl Carbonate-PolyEthylene Glycol-Carboxymethyl (SC-PEG-CM; M.W. 3400 Da). Ormosil nanoparticles were resuspended in de-ionized water (molecular grade) at a final concentration of $0.7 \mathrm{mg} / \mathrm{ml}$ and $10 \mathrm{mg} \mathrm{SC}-\mathrm{PEG}-\mathrm{CM}$ dissolved in $1 \mathrm{ml}$ DI water was added to the Ormosil suspension. The $\mathrm{pH}$ of the mixture was adjusted to 8.0 by addition of diluted sodium hydroxide and the mixture was stirred for 8 hours at $4^{\circ} \mathrm{C}$. PEGylated particles were washed twice using a $100 \mathrm{KDa}$ centrifugation filter to remove free, unreacted PEG monomers. The final product, i.e., pegylated, DOX-IR820 loaded Ormosil (PEGCDSIR820) was stored at $4^{\circ} \mathrm{C}$ until further use. 


\subsubsection{Characterization}

The size and zeta-potential of Ormosil formulations were characterized using Dynamic Light Scattering (DLS) and Scanning Electron Microscopy (SEM). Particles were diluted in DPBS to a concentration of $100 \mu \mathrm{g} / \mathrm{ml}$ and their hydrodynamic diameter was measured in a Malvern Zetasizer ${ }^{\circledR}$. Additionally, nanoparticle morphology was determined using SEM. Nanoparticles were allowed to air dry on a glass slide, sputter coated with gold and imaged using a scanning electron microscope operating at a voltage of $120 \mathrm{kV}$. For measuring the zeta potential, particles were incubated for 1 hour with DPBS or McCoy's 5A medium (with $10 \%$ FBS) at a final concentration of $2.5 \mathrm{mg} / \mathrm{ml}$. The zeta potential was determined using the Smoluchowski approximation and a distribution appropriate for the aqueous medium, i.e., a general distribution for DPBS and monomodal distribution for McCoy's 5A. Six independent batches of nanoparticles were created and evaluated.

Particle composition was studied using FTIR-ATR, Ormosil formulations were air dried at room temperature overnight. Spectra of dried samples was obtained using a Perkin Elmer FTIR-ATR spectrometer.

\subsubsection{Absorbance, Fluorescence and Release kinetics}

Absorbance/fluorescence curves of DOX- IR820 mixture, FDSIR820, CDSIR820 and PEGCDSIR820 particles were obtained in a U-V spectrophotometer or spectrofluorometer (Jobin Yvon). Drug-Dye loading/encapsulation efficiency was determined by DMSO extraction, $250 \mu \mathrm{g} / \mathrm{ml}$ PEGCDSIR 820 particles were dissolved in 
DMSO and IR820 and DOX fluorescence spectra were obtained in a spectrofluorometer. The fluorescence peak values of DOX and IR820 were compared to a calibration of free DOX and free IR820.

CDSIR820 nanoparticles were resuspended in PBS at $250 \mu \mathrm{g} / \mathrm{ml}$ concentration and incubated at $37^{\circ} \mathrm{C}$. Aliquots were drawn at regular time intervals, centrifuged and the IR820 content in the supernatant was determined using fluorescence. Release kinetics of DOX from PEGCDSIR820 was studied in McCoy's 5A media with $10 \%$ FBS. PEGCDSIR820 particles were diluted in media at a concentration of $250 \mu \mathrm{g} / \mathrm{ml}$ and were incubated at $37^{\circ} \mathrm{C}$ under gentle shaking. After one hour of incubation the particles were exposed to $808 \mathrm{~nm}$ NIR laser ( $1 \mathrm{~W}$; fluence rate of $8.3 \mathrm{~W} / \mathrm{cm}^{2}$ for a beam area of $12 \mathrm{~mm}^{2}$ ) for 3 minutes, exposure was repeated every hour for a total of 3 exposures. PEGCDSIR820 were centrifuged at 14,000 r.p.m (2000 g) for 30 minutes using a centrifugal filter and fluorescence intensity of DOX in supernatant was measured. The percentage released drug was calculated using the following formula,

$$
\text { Drug-Dye Loading } \%=\frac{\text { Weight of Drug or Dye in the nanoparticle }}{\text { Weight of nanoparticles }} \times 100
$$

\subsection{Characterization results}

\subsubsection{Size}

Ormosil/silica nanoparticles are formed through a series of steps that involve hydrolysis of the silica precursor followed by condensation and growth of particle nuclei due to intermicellar collisions. The effects of reaction parameters, viz surfactant (dioctyl 
sulfosuccinate sodium salt; AOT), VTES and ammonia/3-aminopropyltriethoxysilane (APTES) concentrations on particle size have been well studied in the literature. The hydrodynamic diameter of three Ormosil formulations is shown in Table 3.1: diameter of Si was $48.5 \pm 2.5 \mathrm{~nm}$, of CDSIR820 was $52.5 \pm 3.0 \mathrm{~nm}$ and FDSIR820 was $52.5 \pm 5.0 \mathrm{~nm}$. The increase in size and polydispersity predicted by dynamic light scattering (DLS) on drug loading can be attributed to the presence of large size fraction particles as shown by the intensity distribution curve (Figure 3.2). It might be possible that drug loading modifies the particle surface and causes minor aggregation of particles in phosphate buffered saline. In aqueous media, physical processes such as aggregation and swelling of organic groups on the nanoparticle surface determine the effective nanoparticle size measured by dynamic light scattering (hydrodynamic diameter). PEG layers on the nanoparticle surface swell in PBS and hence the hydrodynamic diameter of CDSIR820 increased to $58.2 \pm 3.1 \mathrm{~nm}$ from $52.5 \pm 2.5$ (5.7 nanometer increase) after pegylation.

The SEM image shows spherical CDSIR820 particles of uniform size. An agreement between SEM size and average diameter predicted by DLS indicates that the hydrodynamic particle diameter in aqueous media is not increased due to particle swelling and/or aggregation. Addition of PEG improved the aqueous stability of PEGCDSIR820 (compared to CDSIR820) particles as seen from an improvement in the polydispersity index (PDI) (Table 3.1). PEGylated particles exhibited a narrower size distribution (lower PDI) compared to CDSIR820 particles. Both types of particles were stable against aggregation and retained the same diameter over a period of 6 hours. Therefore, the increase in particle diameter was solely due to the PEG-layer and not due to particle 
aggregation. These results are consistent with the pegylation of amorphous silica particles reported by other authors.

\begin{tabular}{|l|l|l|l|l|l|l|}
\hline & & & & Zeta & & \\
& Diameter & & Peta & Potential( & IR820 & DOX \\
Potential(D & Media $)($ & Loading & Loading \\
$(\mathrm{nm})$ & PDI & PBS $)(\mathrm{mV})$ & $\mathrm{mV})$ & $(\mathrm{w} / \mathrm{w} \%)$ & $(\mathrm{w} / \mathrm{w} \%)$ \\
\hline Si & $48.5 \pm 2.5$ & $0.061 \pm 0.015$ & $-40.1 \pm 0.9$ & $-14.6 \pm 2.0$ & N/A & N/A \\
\hline FDSIR82 & $52.5 \pm 3$ & $0.154 \pm 0.022$ & $-39.9 \pm 1.3$ & $-16.9 \pm 1.3$ & $10.0 \pm 0.2$ & $2.1 \pm 0.0$ \\
\hline CDSIR8 & & & & & & \\
\hline IR820 & $52.5 \pm 5$ & $0.214 \pm 0.017$ & $-41.4 \pm 1.9$ & $-17.6 \pm 0.1$ & $6.6 \pm 2.3$ & 0.5 \\
\hline
\end{tabular}

Table 3.1: Physical parameters of different nanoparticle formulations determined by dynamic light scattering. 


\section{Size Intensity distribution of Ormosil Formulations}

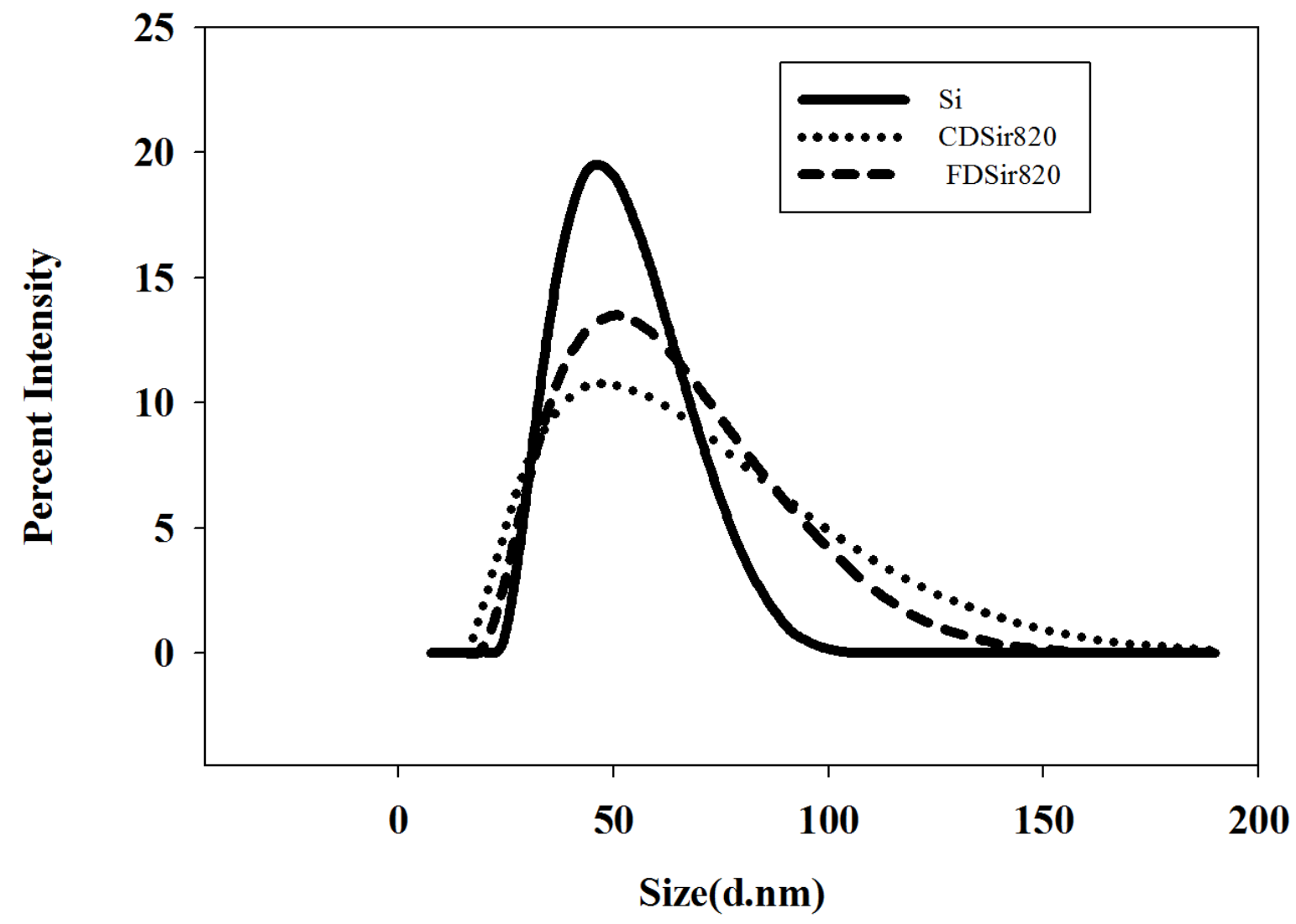

Figure 3.2: Intensity size distribution of Ormosil nanoparticles in Dulbecco's phosphate buffered saline show that the mean diameter of the particles is at $58 \mathrm{~nm}$ 's. 


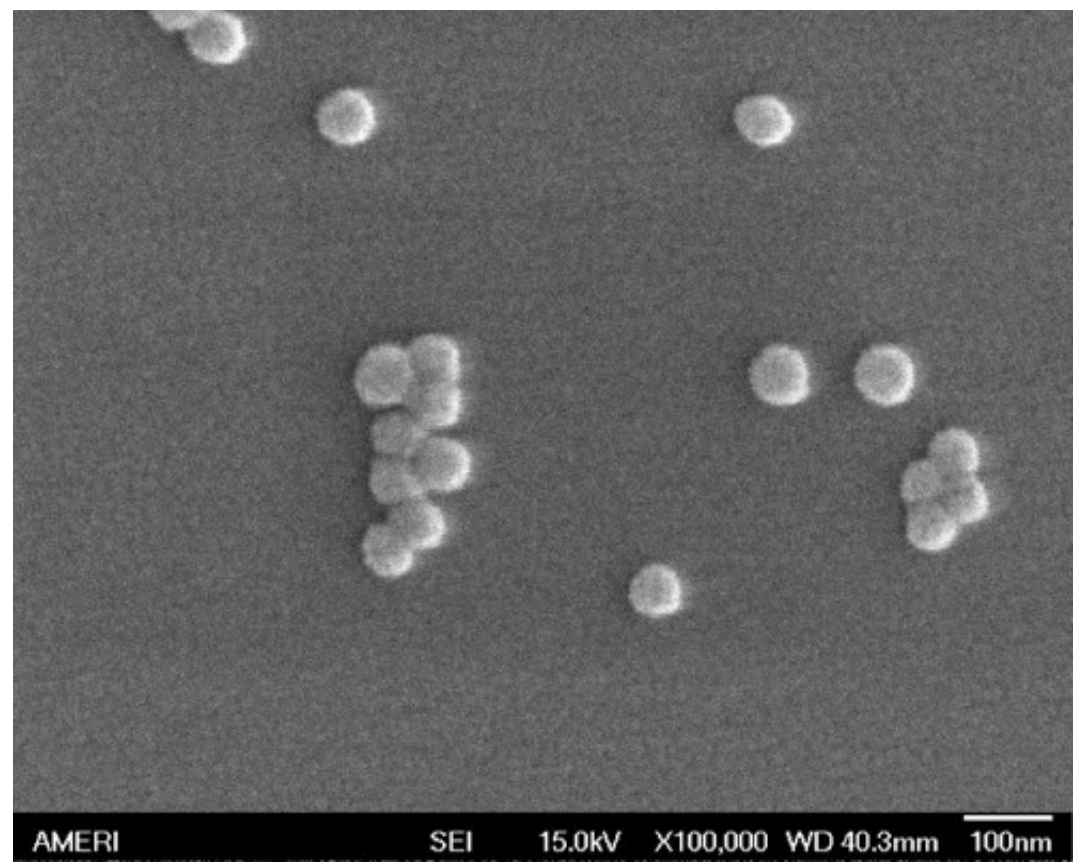

Figure 3.3: Scanning electron microscopy of CDSIR820 particles show monodisperse spherical particles

No aggregation (large aggregates, turbidity) was observed over a period of 6 hours for particles resuspended in DPBS or McCoy's 5A media. Stability against aggregation in physiological buffers/solutions is a desired characteristic for designing nanoparticles for in-vivo applications. Albanese et al. have shown that aggregation results in a lower uptake of nanoparticles by A549 cells (Albanese and Chan, 2011). Also, swelling or aggregation increases the effective nanoparticle diameter which causes a decrease in the diffusion coefficient (diffusion coefficient is inversely related to size) and thereby limits nanoparticle penetration into tumor tissues. The size of our nanoparticles is in the ideal range for optimal cell uptake and deeper penetration into tumor. As cellular uptake of organic/inorganic nanoparticles is an energy dependent mechanism, nanoparticles with diameter less than 30 $\mathrm{nm}$ do not meet the energy threshold needed to drive membrane invagination and wrapping, 
whereas, for particles with diameter greater than $70 \mathrm{~nm}$ the membrane wrapping is slower and more energy intensive (Zhang et al., 2009). Furthermore, nanoparticles with size more than $100 \mathrm{~nm}$ face significant resistance to penetration into tumor tissue due to the presence of tumor extracellular matrix and elevated intratumoral pressure which impedes nutrient and particle diffusion (Goodman et al., 2007).

\subsubsection{Zeta Potential}

Amine grafted Ormosil particles had a zeta potential of $-40.1 \pm 2.3 \mathrm{mV}$ in phosphate buffered saline ( $\mathrm{pH}$ 7.4) (Table 3.1). There are no differences between the zeta potentials of bare $\mathrm{Si}$ and drug loaded formulations owing to the growth of a silica shell over the drug layer. In the case of the Ormosil particles, the zeta potential is not determined by the surface groups such as amine (from APTES functionalization) and vinyl (from VTES monomer), instead, it is determined by the type of surfactant moieties (i.e., Brij-40, Tween-20, AOT) that are immobilized in the silica matrix. Severstel et al. reported that Ormosil nanoparticles prepared with surfactant Brij40 retained the surfactant in silica matrix despite several rigorous washing processes. Their particles had a zeta potential of $-9.3 \mathrm{mV}$ (Selvestrel et al., 2013a).

The zeta potential of CDSIR820 particles shifted to the positive side of neutral on incubation with McCoy's 5A medium containing $10 \%$ FBS. The zeta potential further reduced to $-6.9 \pm 0.07 \mathrm{mV}$ after pegylation (Table 3.1). This is due to the adsorption of serum proteins onto the nanoparticle surface (Lesniak et al., 2010). Adsorption of serum proteins happens very rapidly ( 0.5 minutes $)$ and results in formation of a protein corona around the silica nanoparticle. The protein corona is comprised mainly of proteins with 
molecular mass $>60 \mathrm{KDa}$. Proteins with negative charge seem to preferentially adsorb onto silica nanoparticle surfaces irrespective of the surface zeta potential (i.e., positive or negative). This results in an overall negative charge for Ormosil particles in the presence of serum (Tenzer et al., 2013). Even though PEG provides steric hindrance to protein adsorption it does not completely abolish protein adsorption (Moret et al., 2015a). The protein corona on silica nanoparticles is highly enriched with apolipoproteins involved in lipid and cholesterol transport (Tenzer et al., 2013). Formation of a protein corona on the nanoparticle surface has been shown to be one of the most fundamentally important phenomena in determining nanoparticle interaction with cells (Lesniak et al., 2012).

The cell membrane is an electrically charged surface, therefore the surface charge of nanoparticles also plays a role in cell uptake and tumor penetration. Positively charged nanoparticles are electrostatically attracted to negatively charged cell membranes and taken up by cells to a greater extent compared to particles with negative surface charge. However, particles with positive charge suffer from poor penetration and may also be immobilized due to electrostatic interactions with negatively charged collagen molecules of the ECM (Jain, 1999; Jain and Stylianopoulos, 2010). Similarly, negatively charged nanoparticles aggregate with positively charged hyaluronic molecules. Therefore, a near neutral charge is preferred for efficient tumor penetration and cell uptake. However, colloids are very unstable at their isoelectric point (zero zeta potential) and aggregate in physiological solutions (Oswald's ripening). This can be circumvented by coating the particle surface with PEG (steric hindrance). The mentioned physical properties are ideal for passive accumulation near the tumor site due to tumor EPR. 


\subsubsection{FTIR, Absorbance results}

The FTIR-ATR spectra of Si and CDSIR820 show strong absorption around $1100 \mathrm{~cm}^{-1}$ due to Si-O-Si bridges commonly found in Ormosil particles (Figure 3.4). The dual split peaks in this region are red-shifted by $20-40 \mathrm{~nm}$ to those found in VTES sol gels. This indicates a possible porous structure. Sharp peaks at $1407 \mathrm{~cm}^{-1}$ and $1605 \mathrm{~cm}^{-1}$ indicate in-plane $\mathrm{CH}_{2}$ bending and $\mathrm{C}=\mathrm{C}$ vibrations from the vinyl groups. Furthermore, the peaks at $2969 \mathrm{~cm}^{-1}$ (aliphatic $\mathrm{H}-\mathrm{C}$ ), $3074 \mathrm{~cm}^{-1}$ (aromatic $\mathrm{H}-\mathrm{C}$ ) and at $3400 \mathrm{~cm}^{-1}(\mathrm{~N}-\mathrm{H}, \mathrm{O}-\mathrm{H})$ are due to the functional groups on the surface (Olejniczak et al., 2005; Selvestrel et al., 2013b). The absence of peaks associated with Free DOX or Free IR820 in CDSIR820 samples is because of the additional shell growth on the surface. 


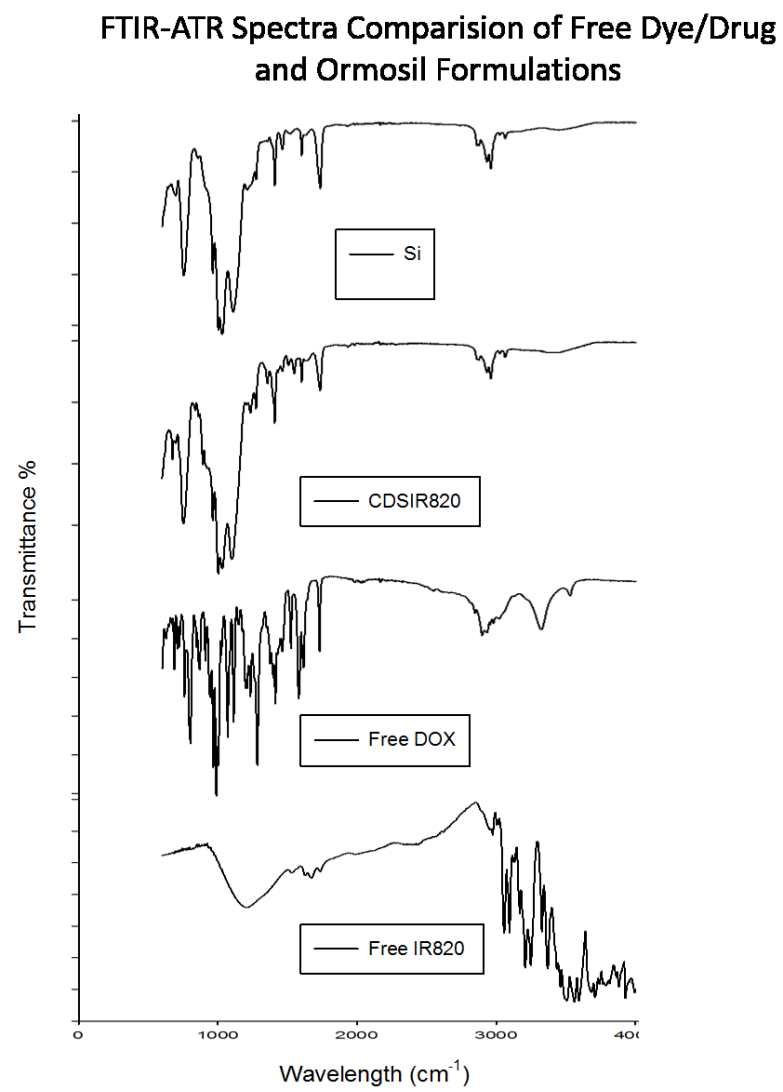

Figure 3.4: FITR-ATR spectra shows core shell structure of CDSIR820. Image shows FTIR-ATR spectra of Blank ORM, CDSIR820, Free Dox and Free IR820. A clear overlap is seen for Blank ORM (no drug or dye) with CDSIR820 indicating the presence of an additional silica shell.

The fluorescence signal of DOX in phosphate buffered saline was quenched for FDSIR820 and CDSIR820 (Figure 3.5). When the particles were incubated with McCoy's 5A media, the fluorescence from FDSIR820 particles increased and reached the expected free DOX value (Figure 3.5), whereas the signal from CDSIR820 remained quenched. Since, DOX is in its hydrophobic form it cannot partition into aqueous media (DPBS) and the proximity to a metal surface resulted in the quenching of fluorescence signal. However, when FDSIR820 particles were re-suspended in cell culture media, DOX was able to partition into the hydrophobic components of the media or was associated with serum 
proteins. This caused an increase in the fluorescence intensity. A similar effect on the fluorescence quenching of DOX was observed by You et al. when DOX was adsorbed onto a hollow gold matrix. The fluorescence subsequently increased on release of DOX from the gold matrix (You et al., 2010).
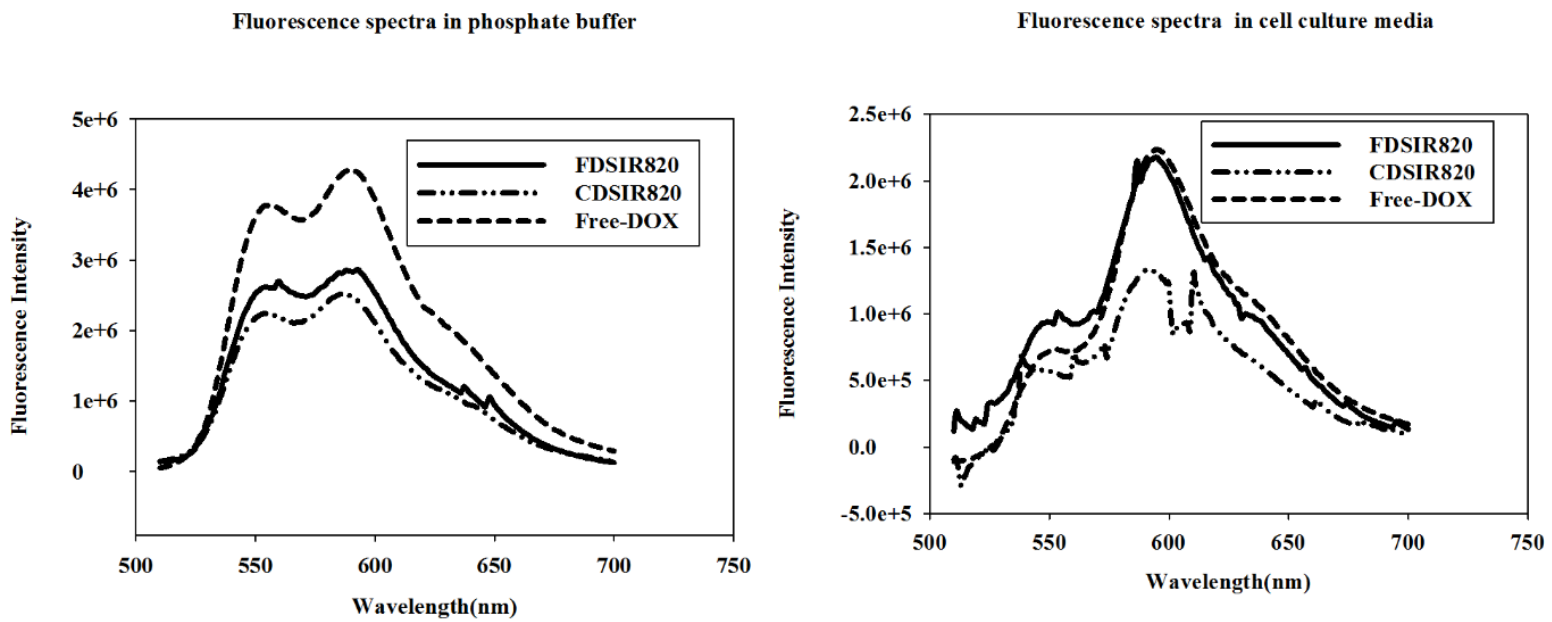

Figure 3.2: Fluorescence studies show leakage of DOX from FDSIR820 in McCoy's 5A media. Fluorescence spectra of different $\mathrm{Si}$ formulations $(3.2 \mu \mathrm{M}$ equivalent DOX concentration) were acquired in DPBS (Left) and McCoy's 5A cell culture media (Right).

Doxorubicin hydrochloride (DOX-HCl) is an amphiphilic drug moiety due to a lipophilic anthraquinone part and a hydrophilic sugar ring. In order to increase the interaction between silica nanoparticles in the oil phase (Dimethyl sulfoxide; DMSO) and DOX molecules, DOX-HCl was converted to its hydrophobic form (DOX) by the addition of Triethylamine (TEA). Hydrophobic DOX molecules are able to partition into the oil phase upon their addition to the emulsion. Furthermore, at high basic $\mathrm{pH}$ of the aqueous phase, most DOX molecules exist in neutral form and are therefore loaded on the silica particle surface via physical adsorption (Raghunand et al., 2003). For the conjugate, 
reaction between DOX and 3-(Triethoxysilyl) propyl isocyanate (ICPTES) results in a DOX-Siloxane intermediate through the formation of urethane (-NHCOO-) or urea (NHCONH-) linkages. However, a urea linkage could preferentially be formed due to the higher affinity of the isocyanate groups $(-\mathrm{N}=\mathrm{C}=\mathrm{O})$ of ICPTES to the amine groups (NH2) of DOX ( $\mathrm{Li}$ et al., 2009). When the DOX conjugate is added to the emulsion, the siloxane groups are hydrolyzed and DOX is covalently loaded onto the silica nanoparticle by condensation of the siloxane groups of the conjugate with the silanol ( $\mathrm{Si}-\mathrm{OH})$ groups on the silica nanoparticle surface.

IR820 was held in the silica matrix due to strong non-covalent electrostatic interactions. The pore size of Ormosil silica nanoparticles is between 1.3-1.7 nm depending on the size of the nanoparticles. The size of the IR820 molecule, based on its molecular weight, is between $1.0-1.3 \mathrm{~nm}$. Since, IR820 is doped at such a high concentration it is possible that IR820 may exist as aggregate molecules which increases their effective size and prevents leakage from the nanoparticles. The absorbance spectra of IR820 shows a strong interaction of the dye with the Ormosil matrix as seen by the red shifted primary peak compared to free IR820 (695 nm's) in PBS. A secondary peak is also observed at 830 nm's from PEGCDSIR820 in DPBS (Figure 3.6). The broadening of absorbance bands is explained from the symmetry breaking and reduced charge delocalization in the polymethine chain of IR820 due to interactions with the Ormosil matrix. Additionally, the $\pi$ - conjugation of terminal end groups has been shown to play a role in the red-shift of absorbance peaks. The resulting broadening of the primary peak $(735 \mathrm{~nm})$ with an appearance of a secondary peak ( $830 \mathrm{~nm}$ 's) is similar to that observed from an elongation 
of middle polymethine chains of NIR dyes by the vinyelene bond $(\mathrm{CH}=\mathrm{CH})$ and extension of nitrogen containing heterocycles (Lepkowicz et al., 2004; Przhonska et al., 2013; Yi et al., 2014).

Absorbance Curves of PEG-CDSIR820 and Free Drug/Dye

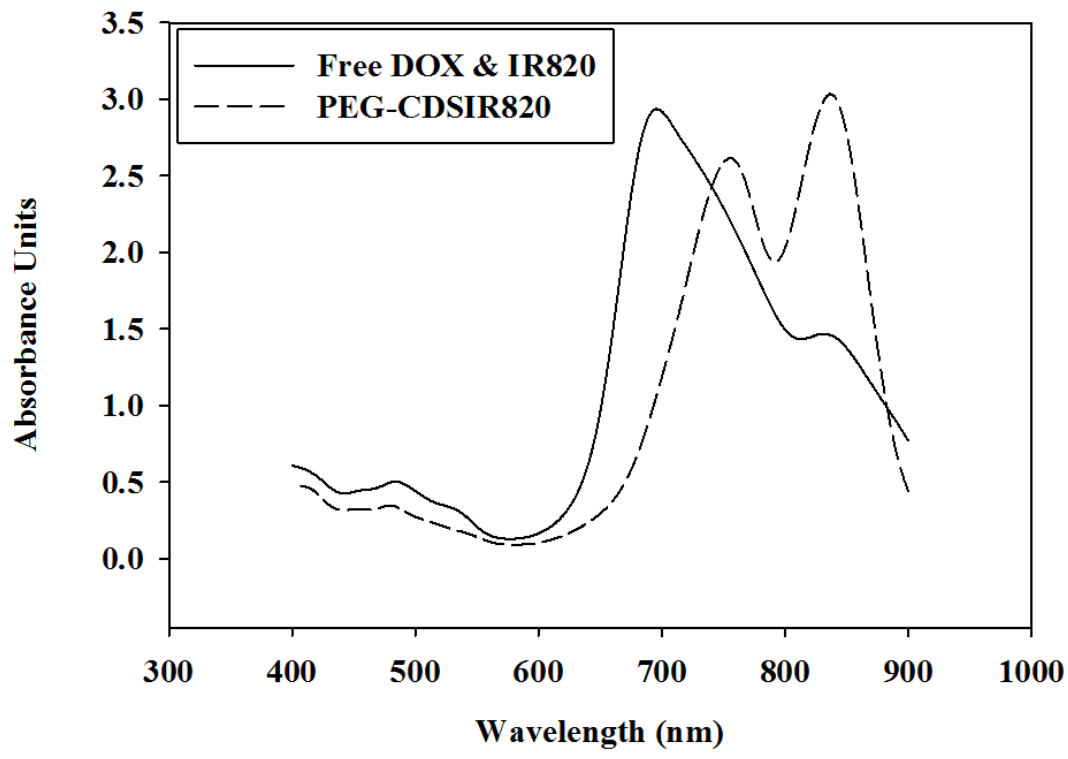

Figure 3.6: Absorbance curves of Free DOX and IR820 mixture in DI water and PEGCDSIR820 in DI water.

For the hyperthermia generation study, the energy fluence rate of a NIR laser was varied in the range of $3.6 \mathrm{~W} / \mathrm{cm}^{2}-6.7 \mathrm{~W} / \mathrm{cm}^{2}$ by changing the output power and keeping the spot size fixed. The energy fluence rate was set at $3.6 \mathrm{~W} / \mathrm{cm}^{2}$ for the first exposure, 4.7 $\mathrm{W} / \mathrm{cm}^{2}$ for the second exposure and was increased to $6.7 \mathrm{~W} / \mathrm{cm}^{2}$ for the third exposure in order to achieve a temperature rise to $43^{\circ} \mathrm{C}$ during each exposure. Under multiple exposures ( 3 exposures were done) to NIR laser, each of duration 3 minutes, the PEGCDSIR820 particles were able to rapidly raise the temperature of the media to $43^{\circ} \mathrm{C}$ 
within 3 minutes for each exposure (Figure 3.7). The temperature measured by the thermocouple at its position in the well is the average of the entire solution temperature. This means that the temperature generated by a fraction of the particles may be higher than $43^{\circ} \mathrm{C}$. After the $3^{\text {rd }}$ exposure, the temperature rise of the media was only $0.5^{\circ} \mathrm{C}$ on exposure to the highest laser power $(1 \mathrm{~W})$, which was the same as observed in media without any PEGCDSIR820 particles. The photobleaching of IR820 due to multiple laser exposures explains the absence of temperature rise after 3 exposures.

Temperature Rise Profile of PEG-CDSIR820

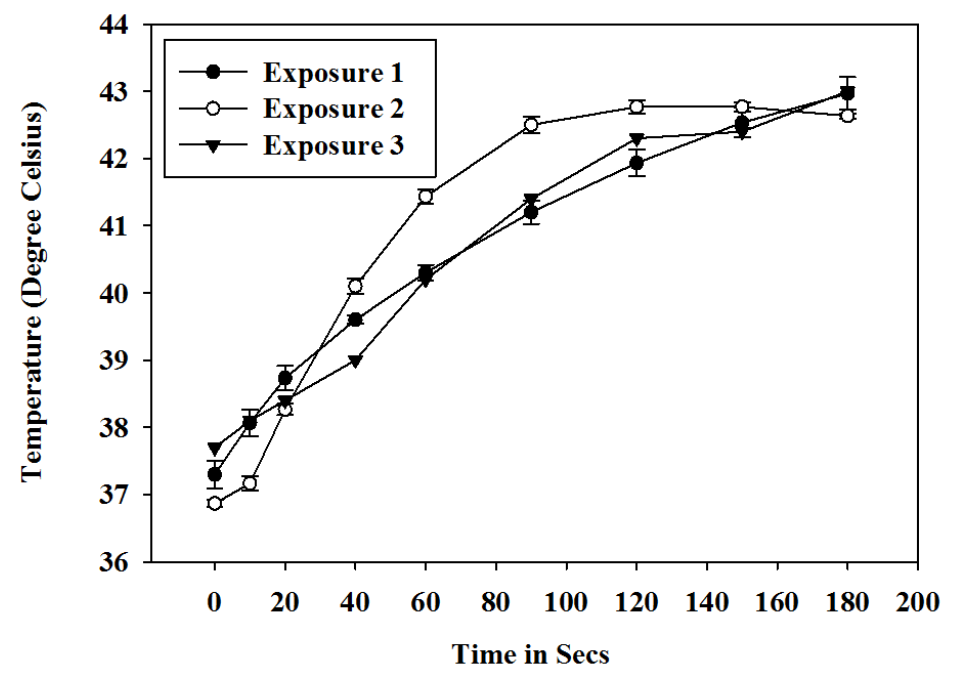

Figure 3.3: Exposure of PEGCDSIR820 to NIR laser resulted in temperature raise. Temperature raise profile of PEGCDSIR820 for successive laser exposures 1 hour apart.

\subsubsection{Release kinetics}

The partitioning of the released DOX is dependent on the release media. In hydrophilic media DOX may not partition out of the particle whereas in cell culture media 
it may partition into the hydrophobic components of the media such as serum proteins. (Roy et.al 2012). shows that DOX and IR820 are slowly released from FDSIR820 and CDSIR820 particles in aqueous media (DPBS). At 48 hours approximately, without any NIR exposure, $93.2 \% \pm 1.5 \%$ DOX was retained in CDSIR820 formulation and $77.0 \% \pm 1.0 \%$ DOX was retained by FDSIR 820 formulation. The slow release of DOX from FDSIR820 is due to the hydrophobic nature of DOX and that of CDSIR820 is due to the gradual hydrolysis of the urea bond in DPBS. A similar release profile was observed for DOX-ICPTES conjugated magnetic particles by Li et al, with only $13.3 \%$ DOX released from the particles in 11 days ( $\mathrm{Li}$ et al., 2009). Release kinetics of DOX from PEGCDSIR820 particles was measured in McCoy's 5A media with 10 \% FBS. Exposure to laser caused the release of DOX from PEGCDSIR820 particles in a time dependent manner. In the absence of laser exposure, only $23 \%$ of DOX was released in 3 hours in McCoy's 5A media (Figure 3.8) with only $5 \%$ observed in the first 20 minutes (burst release). Exposure to the laser resulted in an increase of DOX release from PEGCDSIR820 nanoparticles. Approximately $45 \%$ of DOX was able to release in 3 hours under periodic laser exposure (every 1 hour) (Figure 3.8). At 24 hours, the cumulative release percentage of drug showed no increase from the value measured at 3 hours. The release kinetics of DOX from PEGCDSIR820 was different than un-pegylated CDSIR820 due to the modification of the Ormosil surface with PEG moieties. PEG moieties act as an interface between the hydrophobic DOX molecules and components of media, thus favoring the release of DOX. The mechanism of release under laser exposure is presently unclear, however, hyperthermia and laser exposure may be playing a role in enhancing DOX release from PEGCDSIR820. 

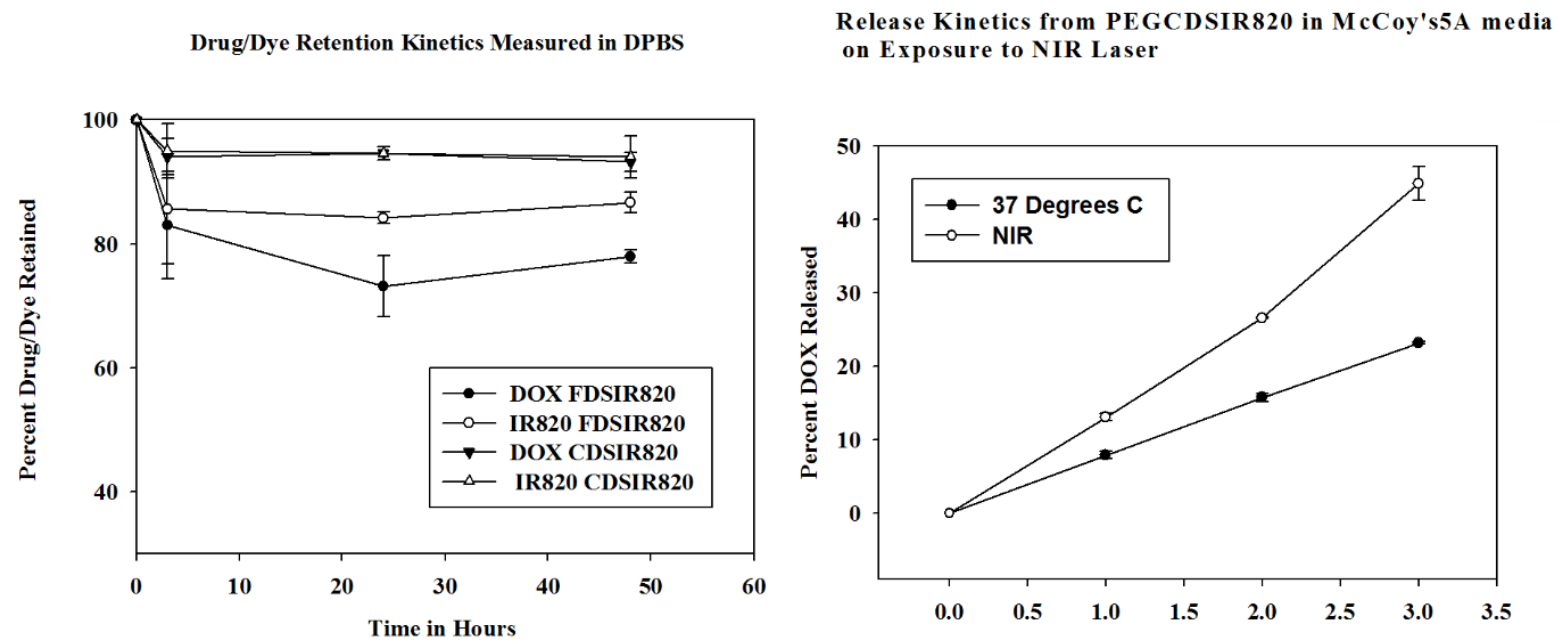
on Exposure to NIR Laser

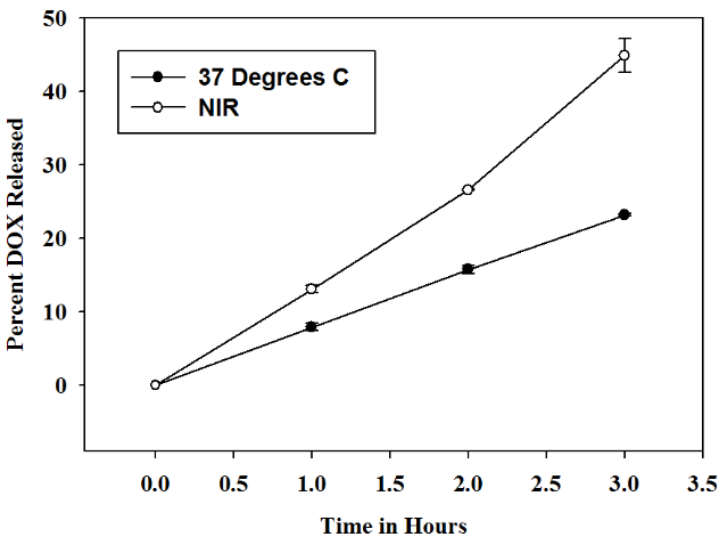

Figure 3.8: Drug (DOX) and Dye (IR820) are retained by ORM formulations in dulbeccos phosphate buffered saline at $37^{\circ} \mathrm{C}$ (left). NIR Exposure of PEGCDSIR820 led to release of DOX. Particles were periodically exposed to laser every hour over a period of 3 hours (right). The error bars represent standard error of mean $(n=3)$.

\subsection{Cell Uptake}

Skov-3 cells were grown on Poly-L-lysine coated coverslips and transfected with Cell BacMam lysolight RFP ${ }^{\circledR}($ Invitrogen). Then, they were incubated with free DOX and DOX loaded nanoparticles (FDSIR820, CDSIR820) for twelve hours, stained with 4',6diamidino-2-phenylindole (DAPI), washed 3 times with ice cold DPBS, fixed with $4 \%$ formalin and mounted onto a glass slide. For laser treatment with PEGCDSIR820 nanoparticles were added to each dish at a concentration of $160 \mu \mathrm{g} / \mathrm{ml}$ and incubated for 24 hours. Cells were exposed to $808 \mathrm{~nm}$ NIR laser (1W) in a manner similar to that described in the cell culture section. After 24 hours, cell nuclei were stained with DAPI for 30 minutes and washed with ice cold DPBS. Cells were fixed in $4 \%$ formalin, washed three times with ice cold DPBS and imaged using a Delta vision Elite deconvolution microscope at the respective wavelengths of the dyes i.e., DAPI ( $\lambda_{\mathrm{ex}}: 360 \mathrm{~nm}, \lambda_{\mathrm{em}}: 430$ 
$\mathrm{nm}), \operatorname{DOX}\left(\lambda_{\mathrm{ex}}: 480 \mathrm{~nm}, \lambda_{\mathrm{em}}: 590 \mathrm{~nm}\right)$ and lyso-RFP ( $\left.\lambda_{\mathrm{ex}}: 550 \mathrm{~nm}, \lambda_{\mathrm{em}}: 610 \mathrm{~nm}\right)$. It should be noted that imaging at DOX wavelengths is only to illustrate the subcellular localization of Ormosil particles, the goal of in-vivo imaging in the NIR region is achieved by IR820 as explained below.

DOX is a DNA intercalating agent that prevents DNA replication by binding with the topoisomerase II complex of cell DNA. Therefore, DOX in its free form is transported to the nucleus which is evident from Figure 3.9 (Free DOX). Similarly, when cells are incubated with FDSIR820 for 12 hours DOX fluorescence is present in the nucleus (Figure 3.9). This is due to labile DOX partitioning into lipid bubbles in the cell membrane due to its hydrophobicity and thus entering the cell and binding with the nucleus in its free form. Conversely, incubation with CDSIR820 resulted in distinct fluorescent puncta in the cell cytoplasm and faint nuclear staining (Figure 3.9). These observations are in accordance with the results by Salvati et al. who showed that the presence of a labile dye on polystyrene nanoparticles results in a diffuse fluorescence, whereas nanoparticles with a non-labile dye appear as distinct fluorescent puncta (Salvati et al., 2011).

DOX fluorescence from CDSIR820 merges with the fluorescence from LysoTracker Red® (Figure 3.9) suggesting that CDSIR820 particles eventually localize in the cell lysosomes. Also, there is a weak DOX fluorescence from the nuclear region (compared to FDSIR820) possibly due to slow urea bond hydrolysis and subsequent release of DOX from CDSIR820 nanoparticles. The cellular uptake mechanisms of Ormosil nanoparticles are not well understood. Studies by Shapero et al. have shown that cell uptake of amorphous silica nanoparticles with similar size $(50 \mathrm{~nm})$ and surface properties (zeta 
potential) is an energy dependent process in which the endocytic uptake mechanisms that are clathirin or caveolae mediated are not involved (Shapero et al., 2011). Fifty nanometer silica nanoparticles enter the cell one by one through membrane invaginations, are subsequently transported to early endosomes and finally get trapped irreversibly in the lysosomes. However, no evidence of clathirin or caveolae was obtained by the authors through short term immunostaining with respective antibodies (Shapero et al., 2011). More recent TEM evidence by Moret et al. of cells incubated with Ormosil nanoparticles showed the presence of clathirin coated pits, however the co-localization of particles within the pits was not found (Moret et al., 2015a). Therefore, it is possible that due to the surface protein corona, silica particles may enter the cell via more specific receptor mediated pathways that are mediated by apolipoproteins. 


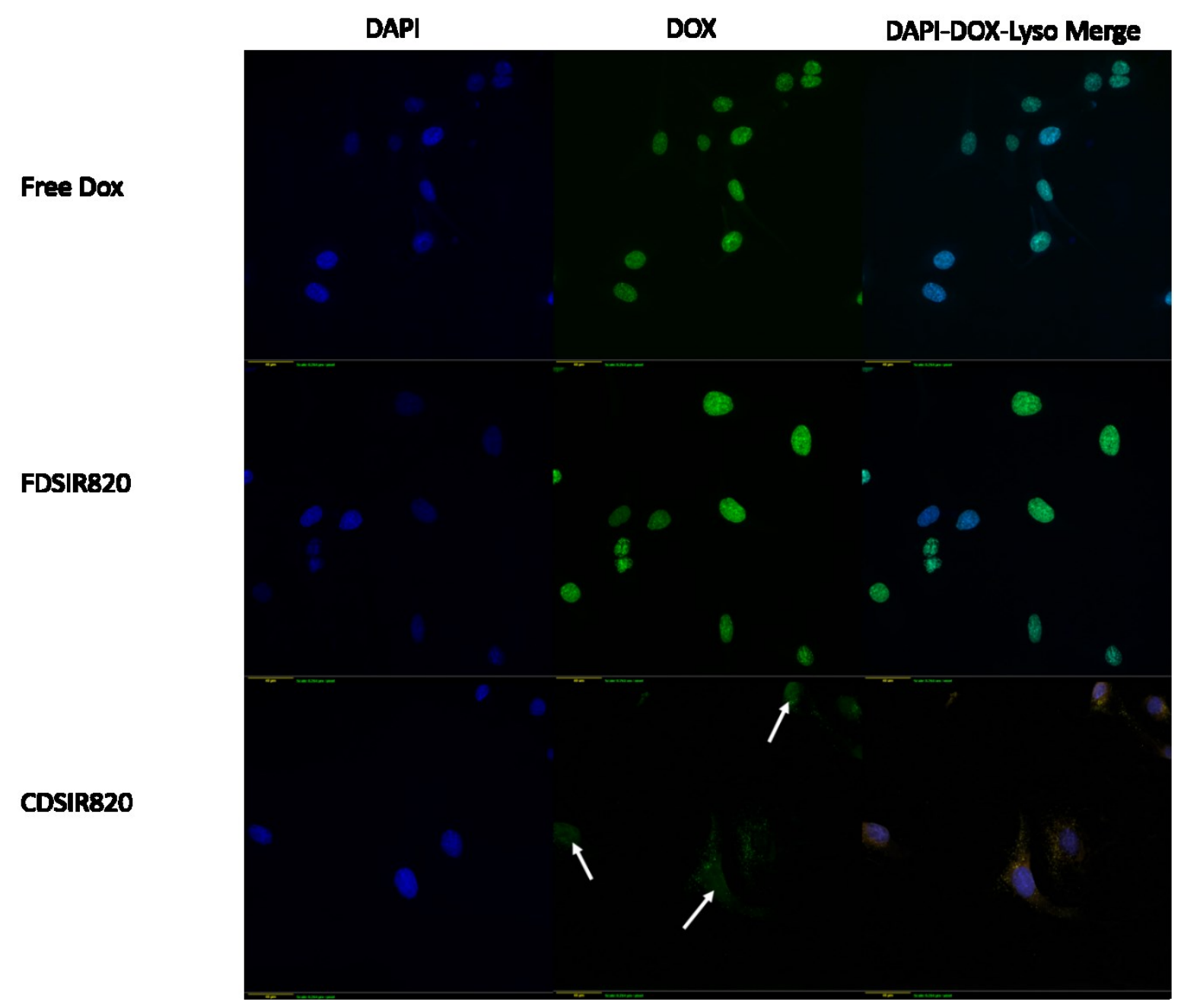

Figure 3.9: Confocal images show differences in intracellular localization of DOX between FDSIR820 and CDSIR820. Fluorescence microscope images of Skov-3 cells incubated with different Ormosil formulations in different fluorescence channels. Scale bar shown in left bottom corner of each picture is $40 \mu \mathrm{m}$. White arrows indicate DOX fluorescence from the nuclear region.

Other transport mechanisms such as phagocytosis or macropinocytosis may also be involved. Further studies are needed to investigate the cellular uptake mechanisms of Ormosil nanoparticles.

Intracellular release of DOX from nanoparticles upon laser exposure is clearly visible. Conditions such as low $\mathrm{pH}$ may be contributing to an increase in DOX release under 
exposure to laser, as seen from an increase in nuclear fluorescence compared to DOX fluorescence in the cytosol. DOX bound to the nanoparticles cannot reach the cell nucleus as nanoparticles are too big to cross the nuclear pore complex. Therefore, upon release from the particles, DOX is able to translocate to the nucleus. Signs of cell necrosis such as fragmentation of nucleus, rounding and shrinking of cells are evident in the PEGCDSIR820 laser treatment group (Figure 3.10).

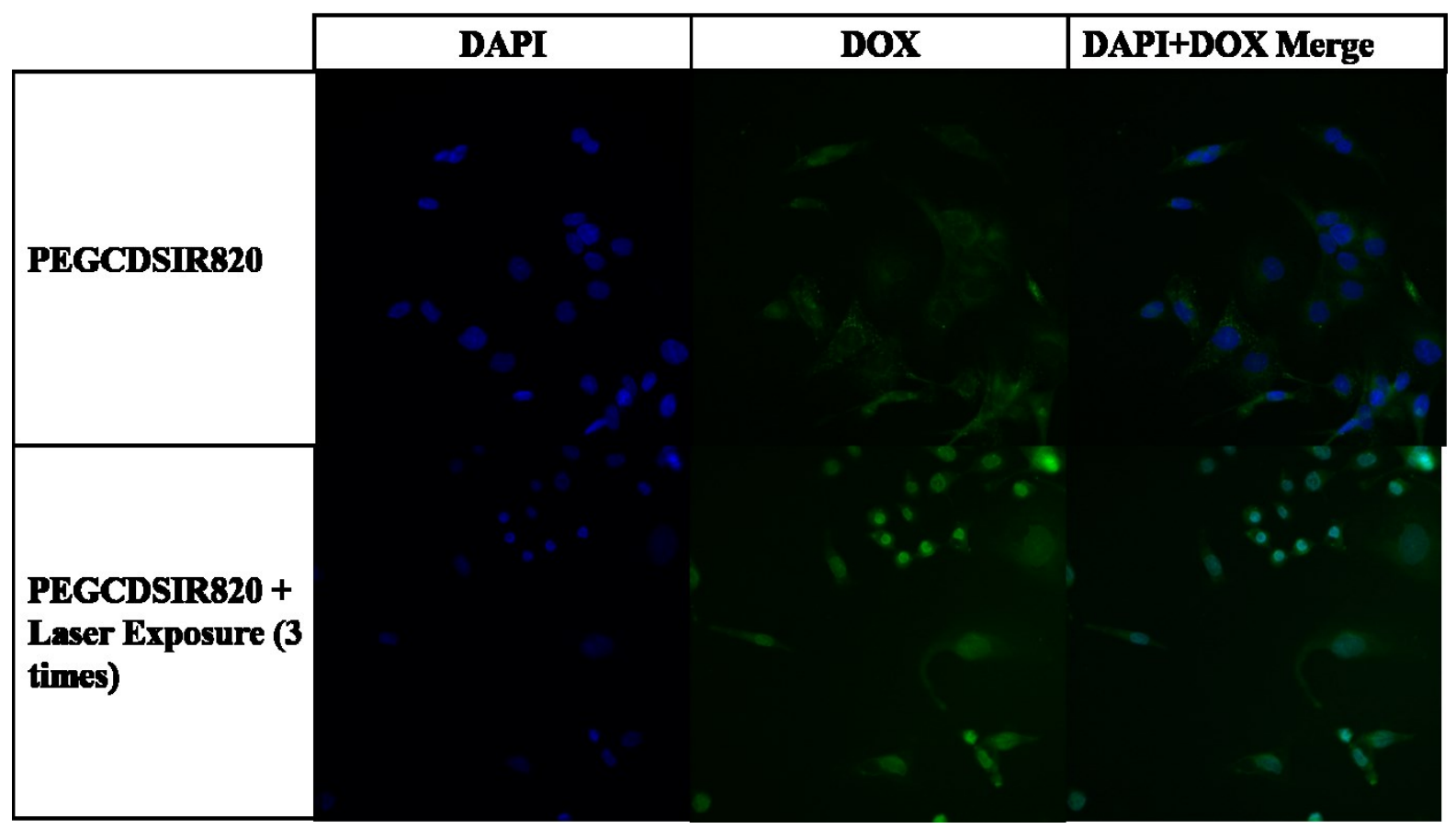

Figure 3.10 : DOX released from PEGCDSIR820 on NIR exposure trans locates to the cell nucleus. Confocal Images of DOX and PEGCDSIR820 distribution in Skov-3 cells. Top panel shows DOX fluorescence from cell nuclei. Middle panel shows fluorescent DOX puncta with minimal fluorescence from the nuclei. Bottom panel shows Skov-3 cells with PEGCDSIR820 particles after laser exposure. DOX fluorescence is evident from cell nucleus. Laser exposed cells show fragmented and shrunk nucleus indicating cell necrosis. 


\subsection{Cell uptake cytotoxicity}

Ovarian carcinoma cells (Skov-3) were maintained in a humidified incubator at $37^{\circ} \mathrm{C}$ and $5 \% \mathrm{CO} 2$. The cells were fed regularly with McCoy's 5A media supplemented with $10 \%$ Fetal Bovine Serum (FBS) and $1 \%$ penicillin-streptomycin. Cellular uptake at $37^{\circ} \mathrm{C}$ and $43^{\circ} \mathrm{C}$ was studied in a cell culture incubator, Skov-3 cells were allowed to equilibrate to $43^{\circ} \mathrm{C}$ for 20 minutes prior to incubation with FITC loaded Ormosil particles for 1 hour at $43^{\circ} \mathrm{C}$ (PEGFITCORM+43). After 1 hour incubation, the cells were returned to $37^{\circ} \mathrm{C}$ for 24 hours. Skov-3 cells were exposed to NIR hyperthermia that was generated by 3-minute laser exposure of $5 \mu \mathrm{M}$ IR820 added to the cell culture media of Skov-3 cells. After NIR exposure, Skov-3 cells were incubated with FITC loaded Ormosil particles for 24 hours (PEGFITCORM+NIR). Cell uptake was determined as mentioned in chapter 2.

For treatment, Skov-3 cells were plated in 96 well plates at a density of 5000 cells per well $(200 \mu \mathrm{l})$ and allowed to attach for 24 hours. Free DOX-HCl, nanoparticles Si, FDSIR820 and CDSIR820, PEGCDIR820 were added to each well at different concentrations $(360,288,216,144,90,36$, or $18 \mu \mathrm{g} / \mathrm{ml})$ and incubated at $37^{\circ} \mathrm{C}$ for 48 hours.

In another group, Skov-3 cells were exposed to $808 \mathrm{~nm}$ wavelength NIR laser (1W) in the presence of PEGCDSIR820 and SIR820 nanoparticles. Each well was exposed to NIR laser for a duration of 3 minutes. A total of 3 exposures with 1-hour interval between successive exposures were done on each well. During laser exposure, the temperature in each well was monitored using a wire thermocouple that was sterilized with $70 \%(\mathrm{v} / \mathrm{v})$ ethanol. After exposure, plates were returned to the incubator. Sulforhodamine B (SRB) 
assay was performed 48 hours after nanoparticle administration to assess cytotoxicity of different treatments. The cells were incubated with nanoparticles for 3 hours and were placed on a heated insert equipped to a movable stage to maintain the cells at $37{ }^{\circ} \mathrm{C}$. The stage was moved so that each well to be exposed was directly in-line with the laser probe. The exposure time for each well was 3 minutes, which was based on the previous studies. Temperature of the well was monitored during laser exposure by a wire-thermocouple. After laser exposure, the plates were incubated for 48 hours and cytotoxicity was determined using the SRB assay. Three experiments with 3 replicates each were performed. Exposure of cells to rapid rise hyperthermia is detrimental to cell uptake of nanoparticles. Therefore, the timing of hyperthermia is crucial.

\subsubsection{Cytotoxicity of Different Ormosil Formulations in Skov-3 cells}

The cytotoxicity results in Figure 3.11 show that Si and CDSIR820 nanoparticles are well tolerated by Skov-3 cells and no growth inhibition is observed below $150 \mu \mathrm{g} / \mathrm{ml}$ nanoparticle concentration. Meanwhile, FDSIR820 nanoparticles showed severe toxicity at relatively lower concentrations compared to the Si and CDSIR 820 formulations. Ninetyfour percent growth inhibition was observed in Skov-3 cells when administered $150 \mu \mathrm{g} / \mathrm{ml}$ FDSIR820 nanoparticles. These results are in accordance with Roy et al. who observed a significant reduction in cell viability $(\sim 80 \%)$ of $\mathrm{MiPaCa}-2$ pancreatic cells treated with DOX loaded Ormosil particles (Roy et al., 2014). The rapid release of DOX from FDSIR 820 and binding with the nucleus might explain the severe toxicity caused by this formulation. Premature rapid release of drug from nanoparticles is disadvantageous to invivo applications as it will cause systemic toxicity associated with the drug and may also 
reduce available injected dose of DOX to the tumor. The observed toxicity in CDSIR820 was lower compared to the toxicity of free DOX at an equivalent DOX concentration of 5 $\mu \mathrm{g} / \mathrm{ml}$. The lower cell killing potency of CDSIR820 compared to free DOX is due to the slow release of DOX from the particles.

The observed toxicity profile for Si particles is in accordance with Moret et al. who have shown that unpegylated blank Ormosil nanoparticles were well tolerated by NCIH2347 adenocarcinoma and CCD-34Lu normal fibroblast cells up to a concentration of 200 $\mu \mathrm{g} / \mathrm{ml}$ (Moret et al., 2015b). The toxicity of Ormosil nanoparticles in cancer cells is dependent on cell type and relies on disruption of various vital cell functions. Ormosil nanoparticles have been shown to induce structural changes in cell morphology, alter cell membrane permeability and metabolism, cause the generation of reactive oxygen species which damage the mitochondria (Figure 3.12) and promote inflammatory gene expression.
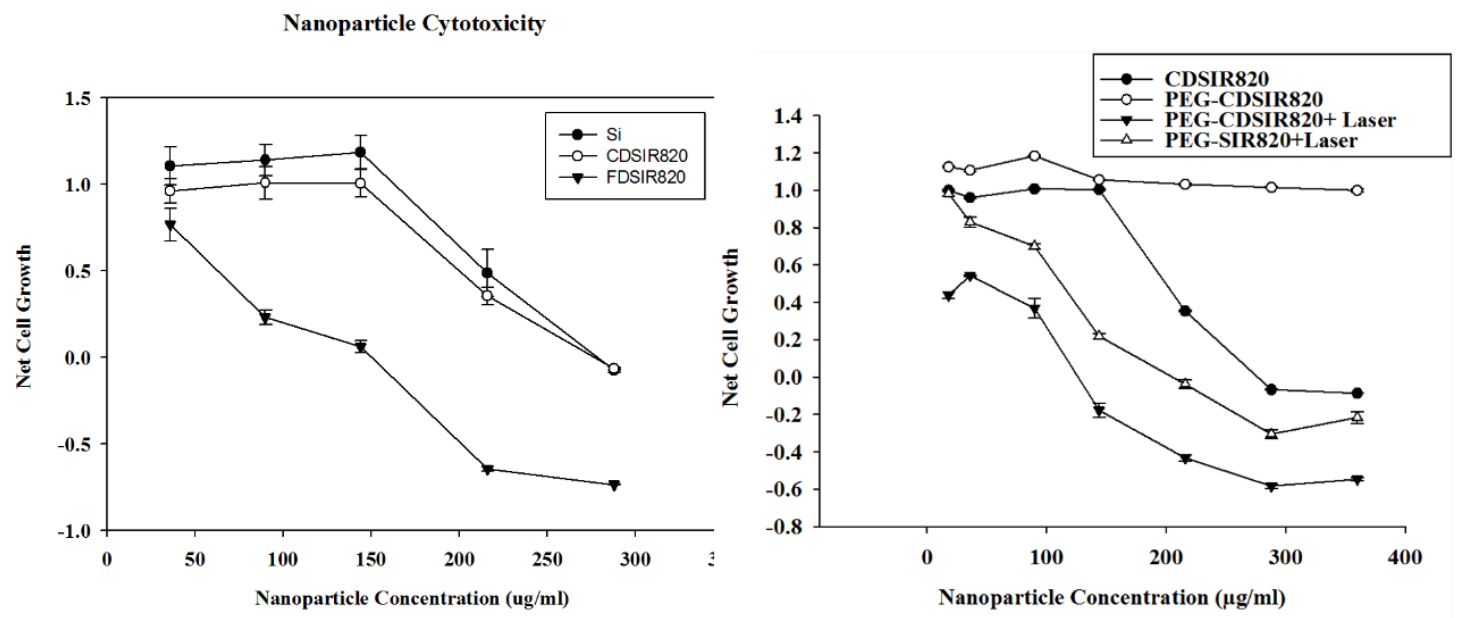
Figure 3.4: Dose dependent cytotoxicity of different nanoparticle formulations under presence or absence of laser exposure. Comparison of FDSIR820 and CDSIR820 (left). Effect of Pegylation and laser exposure on cytotoxicity of PEGCDSIR820. The error bars represent standard error of the mean $(n=3)$.

DOX and IR820 loaded PEGylated particles were well tolerated by Skov-3 cells at higher doses compared to un-pegylated particles, inhibition of cell growth or cell killing was not observed at the highest tested concentration of PEGCDSIR820 (Figure 3.11). This is in accordance with recent results reported by Moret et al. which showed that pegylation of Ormosil nanoparticles reduced toxicity in lung cells. Altered cell uptake due to addition of PEG may also be contributing to the reduction in toxicity (Moret et al., 2015b). Uptake of PEGCDSIR820 by Skov-3 cells is clearly visible by the fluorescent puncta observed from confocal images (Figure 3.11). Exposure to NIR laser markedly increased the toxicity of PEGCDSIR820 nanoparticles; $60 \%$ inhibition of cell growth was observed at the lowest tested concentration (18 $\mu \mathrm{g} / \mathrm{ml})$. PEGylated Ormosil nanoparticles loaded only with IR820 (PEGSIR820) also showed enhanced toxicity on laser exposure, however, they were less toxic compared to PEGCDSIR820 (Figure 3.11). Signs of cell necrosis, such as fragmentation of nucleus, rounding and shrinking of cells, are evident in the PEGCDSIR820 laser treatment group (Figure 3.11). The release of DOX and multiple rounds of hyperthermia generated by laser exposure explains the enhanced toxicity of PEGCDSIR820 particles. Skov-3 cells are intrinsically insensitive to DOX chemotherapy due to a p53 gene mutation. This implies that increasing the intracellular content of DOX is not sufficient to induce higher cell killing. Adjuvant hyperthermia is beneficial for inhibiting p53 deficient tumor cells as it has been shown that the cellular response to 
hyperthermia is independent of p53 status. Moreover, rapid rate, short term hyperthermia does not lead to expression of heat shock proteins (HSP) which is commonly observed in slow rate long term hyperthermia ( $>=1 \mathrm{hr}$.) (Tang and McGoron, 2009; Tang, 2010). Over expression of HSP is associated with an increase in thermal tolerance and has a positive correlation with an increase in drug resistance (Comerford et al., 2002). Since, a high thermal dose can be delivered on-demand multiple times, greater cell killing efficiency can be achieved than through DOX therapy alone. This is advantageous from a clinical treatment perspective where repeated treatment cycles are often needed to achieve complete eradication of tumors.

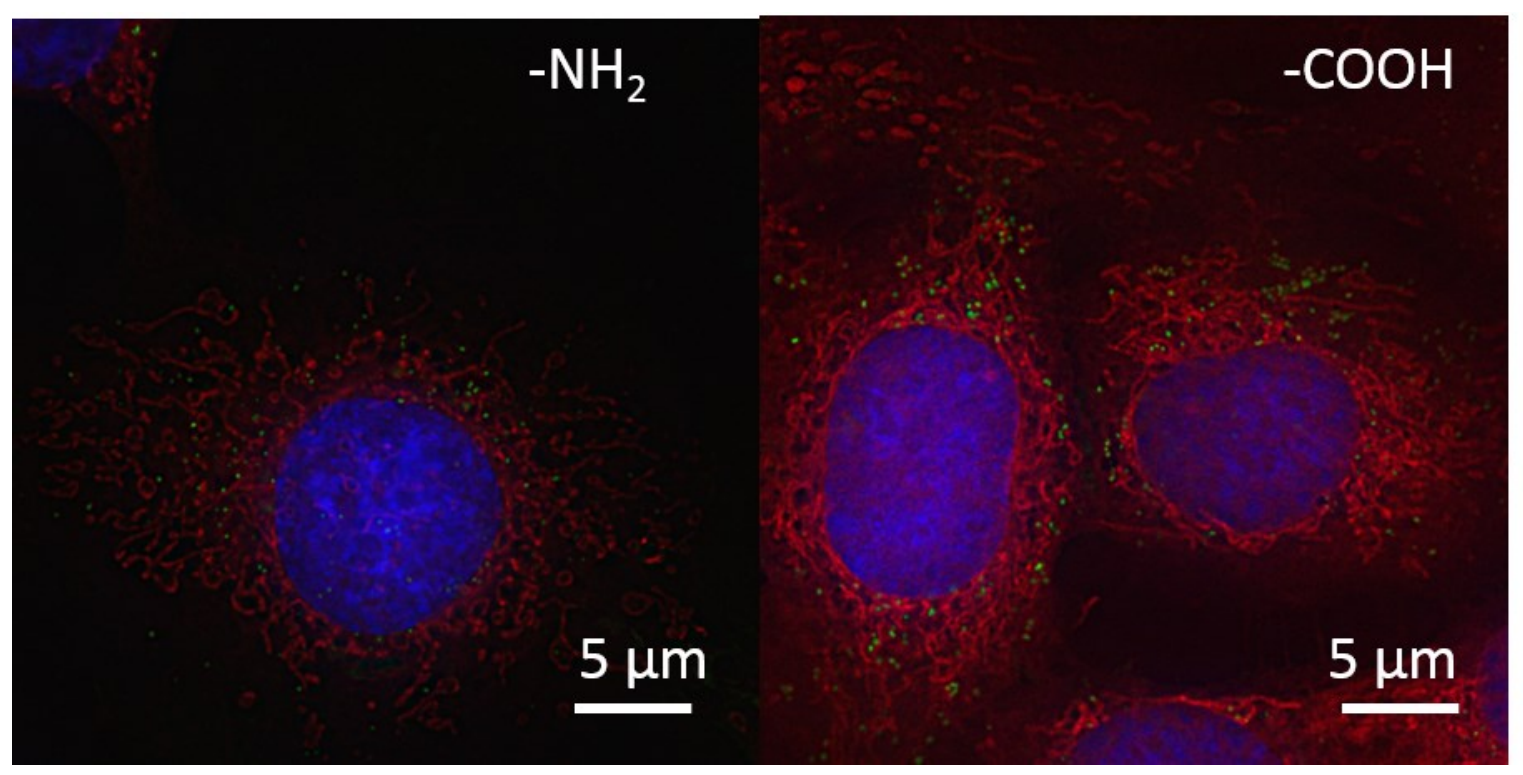

Figure 3.5: Silica nanoparticles affect the cell mitochondria. Cells were stained with Mitotracker Red as per manufacturer's instructions. Green puncta indicate nanoparticles. Cells incubated with aminated silica particles show fragmented mitochondrial cristae (left). Carboxylated silica nanoparticles did not induce mitochondrial fragmentation (right). Images were taken with $100 \mathrm{X}$ objective using the DeltaVision ${ }^{\circledR}$ deconvolution Microscope. 


\subsection{Spheroid Studies}

Spheroids were cultured and characterized as described in Chapter 1. Spheroids were transferred to $200 \mu \mathrm{l}$ fresh media in a 96 well plate, PEGCDSIR820 particles and DOX were added at a final concentration of $250 \mu \mathrm{g} / \mathrm{ml}$. In one study, the spheroids were incubated with PEGCDSIR820 particles for 2 hours and exposed to laser in a manner similar to cell monolayers. In another study, spheroids were incubated for 24 hours and exposed to laser 3 times. The number of viable cells in spheroids was assessed by trypan blue assay 24 hours after laser exposure. Spheroids were disaggregated by trypsin, centrifuged and resuspended in $30 \mu \mathrm{DPBS}$. An equal volume of trypan blue was added and viability was determined using a hemocytometer as the ratio of trypan blue positive cells to total cell count. For each treatment group eight spheroids were used. For imaging, spheroids were incubated with $250 \mu \mathrm{g} / \mathrm{ml}$ PEGCDSIR820 nanoparticles for 2 hours and exposed to laser 3 times. After 8 hours, spheroids were incubated with DAPI for 1 hour, washed in ice cold DPBS and fixed in 4\% formalin. Confocal sections of $10 \mu \mathrm{m}$ thickness were obtained using an Olympus FV1200 confocal microscope.

Tumor spheroids recreate functional and morphological conditions of in-vivo avascular tumors. Dense aggregation of tumor cells and ECM molecules are a barrier to delivery of oxygen and nutrients. Hence, tumor tissues have a stratified organization of cell layers (i.e., necrotic core, quiescent layer and a proliferating cell region). As noted earlier, cells in these quiescent regions have a different physiological response to therapy, i.e., they are more resistant to therapy due to the tumor microenvironment (hypoxia, low $\mathrm{pH}$ ). At the same time, a barrier to molecule diffusion is a limitation for the transport of nanoparticles. 
Confocal images obtained after 8 hours' incubation show that free DOX was able to distribute homogeneously in the spheroids, whereas PEGCDSIR820 particles remained at the periphery of the spheroids (Figure 3.13). The diffusion coefficient of free DOX is much smaller compared to PEGCDSIR820 particles (as diffusion coefficient is inversely related to the square of effective particle diameter). Additionally, the effective diameter of particles may have increased due to swelling and protein adsorption resulting in slow diffusion through the spheroid.

NIR exposure increased the nuclear fluorescence of DOX as seen from the merged image of DAPI and DOX (Figure 3.13). However, after 8 hours, spheroids from both PEGCDSIR820 and PEGCDSIR820 laser treatment groups did not show any visible signs of toxicity, i.e., disintegration of spheroids, and the detachment of cells from the periphery that are commonly seen in treated spheroids. In cells exposed to NIR hyperthermia (by adding 5 $\mu \mathrm{M}$ IR820) uptake of FITC-loaded nanoparticles was reduced compared to uptake at $37^{\circ} \mathrm{C}$ and $43^{\circ} \mathrm{C}$ (Figure 3.14). Additionally, PEGORM particles aggregated on exposure to NIR laser as observed by the formation of visible particle aggregates. Exposure of cells to NIR induced hyperthermia from IR820 leads to cell membrane damage (Tang et al 2009). These effects contribute to the reduction in the cellular uptake of PEGORM nanoparticles. Therefore, the proper timing of hyperthermia delivery is needed to obtain the combined effect of DOX and hyperthermia treatments. Furthermore, the aforementioned results were obtained by incubating the spheroids for 8 hours with PEGCDSIR820. Eight hours is sufficient to saturate the cell uptake of nanoparticles in monolayers (Chapter 2). However, in spheroids this process is considerably slowed as 
particles have to overcome the physical barrier to reach cells deep within the spheroid. At this time, it is not possible to accurately model the particle penetration under hyperthermia due to the presence of DOX release from the particles. The simulation strategy needs to account for drug release and subsequent transport through the spheroid which is out of the scope of present study. Moreover, cells in quiescent and hypoxic regions may display altered uptake mechanisms of nanoparticles. We have observed that cells subjected to long term hypoxia display autophagic uptake of silica nanoparticles. Figure 3.15 shows staining with Monodansylcadaverine (MDC) that preferentially accumulates in autophagic vacuoles via ion trapping and specific interactions with membrane lipids. 


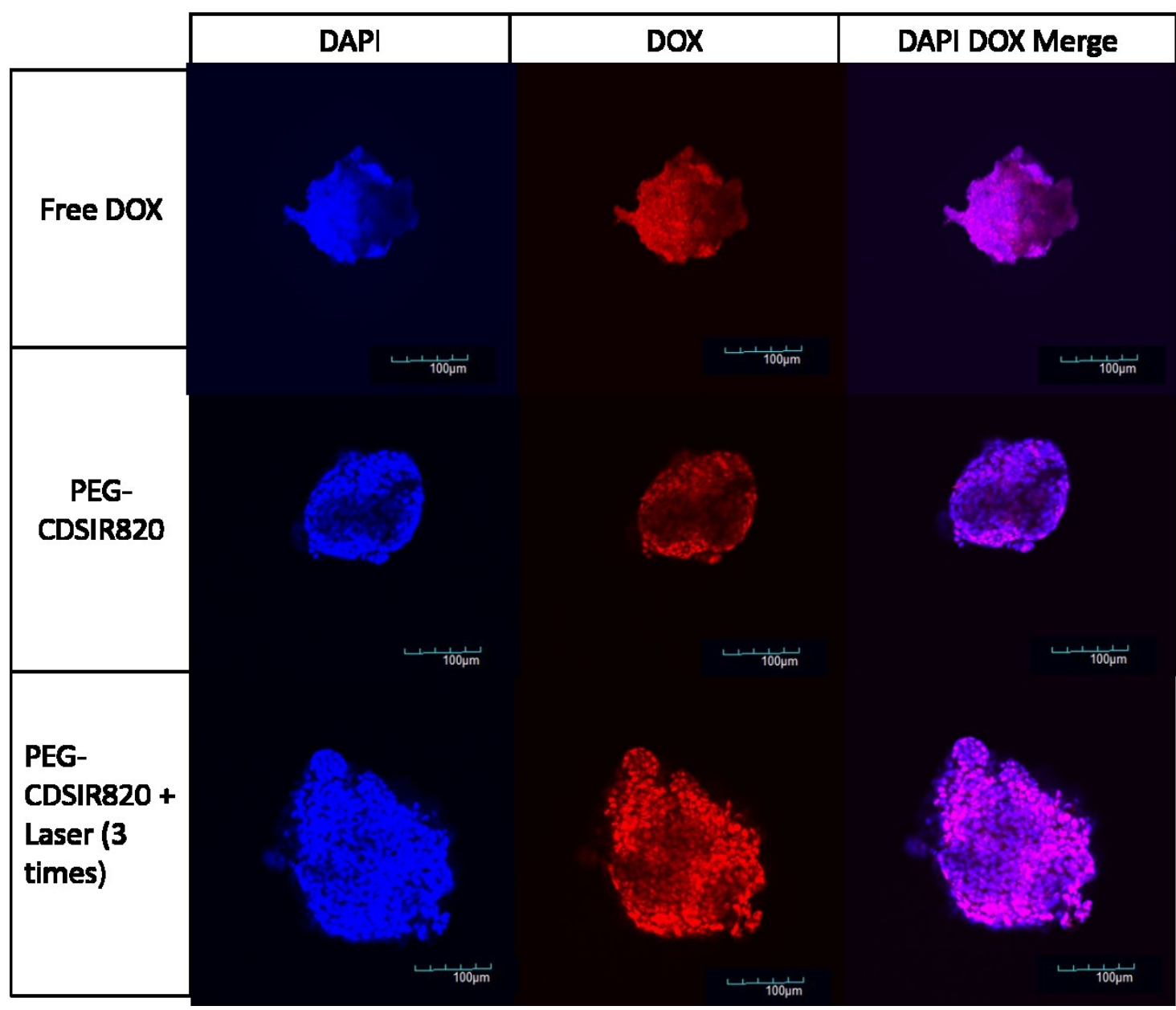

Figure 3.13 : Confocal Images of $90 \mu \mathrm{m}$ deep sections of Skov-3 spheroids from 2-hour study in which spheroids were incubated with PEGCDSIR 820 for 2 hours and then exposed to laser 3 times. Images were collected after 8 hours. Top panel is the image of PEGCDISR820 particles with no laser exposure. DOX fluorescence is concentrated around the periphery. Bottom panel shows image of PEGCDSIR820 particles with laser exposure. DOX and DAPI fluorescence is more diffuse in the spheroid following laser exposure compared to the no-exposure group. Scale bar is $100 \mu \mathrm{m}$. 
Effect of Hyperthermia On cell uptake of ORM particles

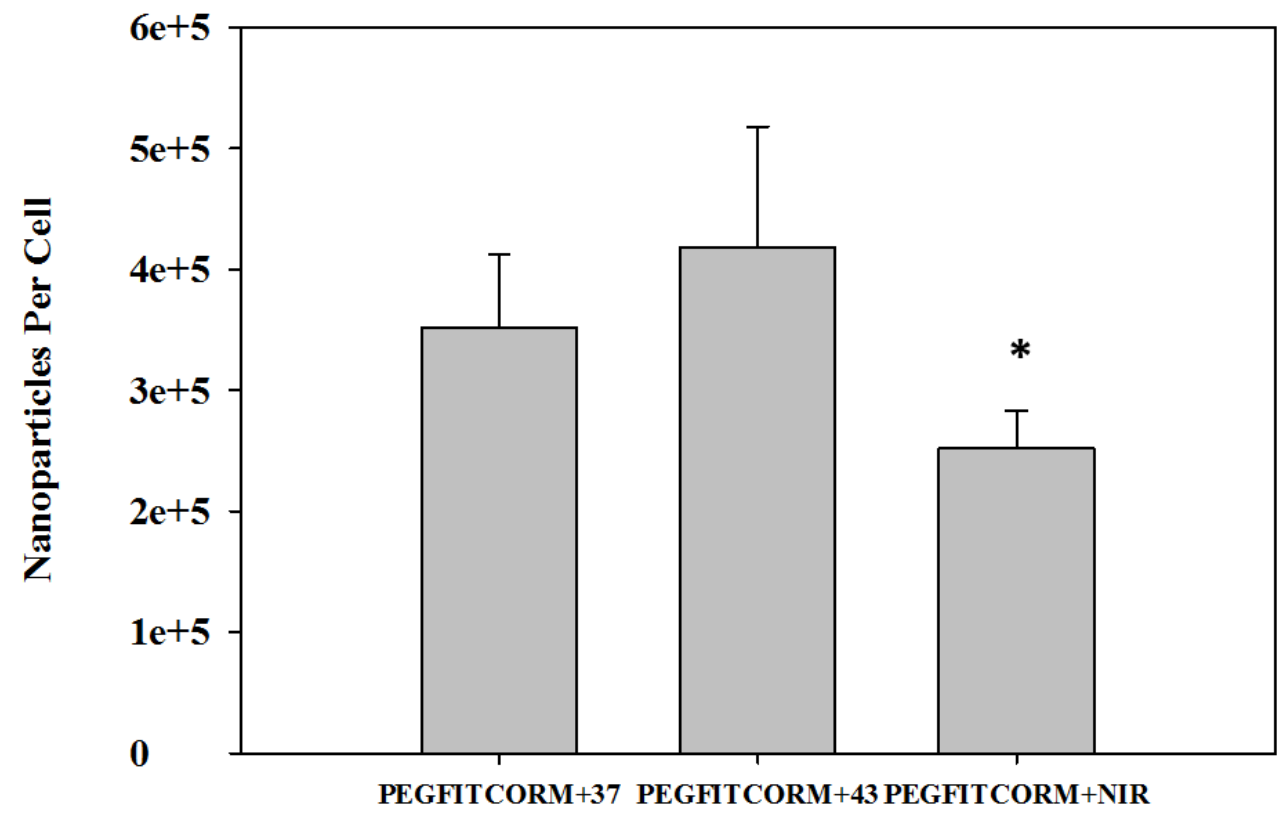

Figure 3.14: Exposure to NIR hyperthermia reduced the cell uptake of PEGFITCORM nanoparticles. Uptake of PEGylated Fluorescein Isothiocyanate loaded Ormosil particles (PEGFITCORM) by Skov-3 cells after 24 hours under different temperature conditions (left), (*) represents significant difference between uptake at $37^{\circ} \mathrm{C}(\mathrm{PEGFITCORM}+37)$ and NIR exposure (PEGFITCORM+NIR). 
Figure 3.15 : Evidence of autophagic uptake (different uptake mechanism compared to endocytosis) of silica nanoparticles under hypoxia. Cells were subject to $1 \% \mathrm{PO}_{2}$ (Hypoxia) for $18 \mathrm{hrs}$. followed by incubation with Si nanoparticles for 24 hours. Autophagy was imaged by staining cells with Monodansylcadaverine.

NIR laser after 24 hours incubation with PEGCDSIR820 nanoparticles led to complete disintegration of the spheroid mass the following day (24 hours) (Figure 3.16). This was further supported by a viability test that showed $94 \%$ trypan blue positive cells in the laser treatment group (Figure 3.16) and are also in accordance with the predictions from finite element simulations. Spheroids incubated with PEGCDSIR820 nanoparticles also showed a reduction in cell viability which is in contrast to results observed in monolayer cell cultures that showed no toxicity to PEGCDSIR820 at the tested concentrations. This is possible since it has been shown by Lee et al. that Skov-3 cells grown as spheroids show a reduction in the corresponding p-53 mutation observed in monolayer culture (Lee et al., 2013). The lack of p-53 mutation, in combination with oxygen, nutrient deprivation and DOX may be causing cytotoxic stress and cell death in the quiescent regions of the spheroid. 

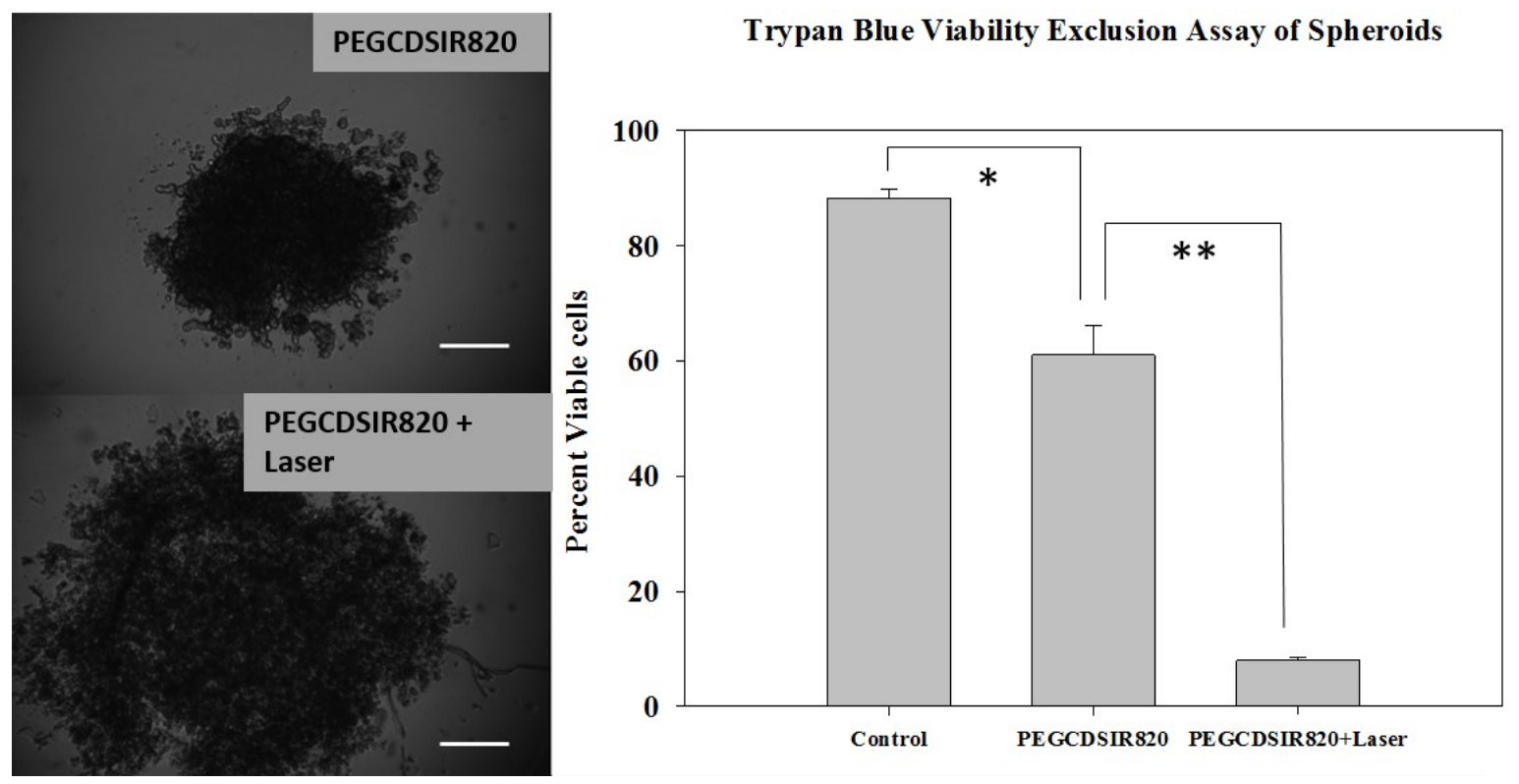

Figure 3.16: Exposure to NIR hyperthermia of PEGCDSIR820 lead to spheroid toxicity.Top left image shows Skov-3 spheroids incubated with PEGCDSIR820, the compact structure is apparent with some loose cells at the periphery. Bottom left shows a less compact spheroid as a result of cell dissociation and death due to PEGCDSIR820 + Laser treatment (scale bar is $100 \mu \mathrm{m}$ ). Trypan blue viability results for spheroids from the 24-hour study. Spheroids were incubated with PEGCDSIR820 for 24 hours and then exposed to laser 3 times. Trypan blue assay was done 24 hours after laser exposure. Data is represented as Mean $\pm \mathrm{S}$.E $\mathrm{n}=8,(*)$ indicates significance for $\mathrm{p}<0.05$ and $(* *)$ indicates significance at $\mathrm{p}<0.001$ level.

The difference between the appearance of spheroids that were treated with DOX or DOX + HYP (Chapter 1) and PEGCDSIR820 has to be noted. While, spheroids treated with DOX or DOX + HYP showed a loss of cells from the periphery (also observed in PEGCDSIR820) and intact spheroid mass on Day 6; spheroids with PEGCDSIR820 exposed to laser showed complete disintegration and diffuse structure 24 hours after the treatment. 


\subsection{Biodistribution Studies.}

ICR mice were bred in house and kept under standard housing conditions, and fed ad libitum. All protocols followed the regulations of the Institutional Animal Care and Use Committee. Mice were randomly assigned to different experimental groups based on different time points, namely 15 minutes, 30 minutes, 60 minutes, 6 Hours, 24 hours and 48 hours. On the day of the experiment, the animals were anesthetized with isofluorane and injected i.v. through the tail vein with a solution of NPs in PBS. The concentration of injected NPs was determined based on an IR820 dose of $0.24 \mathrm{mg} / \mathrm{kg}$ of body weight and an injection volume of $0.1 \mathrm{~mL}(1 \mathrm{mg} / \mathrm{ml}$ Ormosil concentration). At the terminal time point for all groups (15 min, $30 \mathrm{~min}, 60 \mathrm{~min}, 24 \mathrm{~h}$ and $48 \mathrm{~h}$ ), the animals were euthanized and their organs were harvested. Blood samples were collected via a heart-stick. Twenty microliter blood drops were placed on a glass slide and fluorescence images were obtained in a Li-Cor ${ }^{\circledR}$ Odyssey imaging system. Region of interests were drawn around the droplets and fluorescence intensity was obtained. The fluorescence intensity was compared to a calibration plot (intensity vs particle concentration) obtained from serial dilutions of PEGCDSIR820 in mouse blood.

IR820 fluorescence from the organs and blood was also measured using the LiCorß Odyssey imaging system. Region of interests were generated for each organ and the fluorescence intensity was determined. Fluorescence intensity of each organ was converted to particle concentration in a similar manner as described above. The particle concentration was normalized with respect to organ weight in grams. 
The fluorescence intensity of animal organs was measured following intravenous administration of CDSIR820 particles using an NIR imaging system operating at $800 \mathrm{~nm}$ wavelength. Strong IR820 fluorescence signal was obtained from the excised organs. From the calculated injected dose, it is clear that a major fraction of the injected dose of the nanoparticles accumulate in the organs of the reticuloendothelial system (RES), including liver, lungs, kidneys and spleen. In the first 24 hours, the injected dose peaks at 30 minutes in liver and kidneys (Figure 3.17). There are two modes of sequestration of nanoparticles in the RES organs, 1) passive accumulation and 2) capture by the macrophages residing in these organs. The sinusoidal wall of the liver has discontinuous gaps in its endothelium which might result in passive accumulation of nanoparticles. The accumulation in spleen increases remarkably at 24 hours, possibly due to increased capture of nanoparticles by the macrophage cells present in the organ.

The kinetic distribution profile in the kidneys up to 24 hours may suggest some clearance via urine, however, the size of the nanoparticles is above the threshold for glomerular filtration and subsequent excretion via kidneys. Only particulates in the size range $7-10 \mathrm{~nm}$ are efficiently excreted via the kidneys (Ernsting et al., 2013). For particles above $30 \mathrm{~nm}$ diameter, excretion in feces via hepatobiliary clearance is a more prominent mode of removal. Since we do not expect our Ormosil to be degraded or metabolized rapidly, hepatobiliary clearance is more probable than renal clearance. The presence of particles in heart is somewhat surprising, this may be possible since silica nanoparticles have been shown to associate with vascular cells. This may explain the variation observed in heart tissue, which might have been caused due to the presence of residual blood clotted 
in the organ following euthanasia. Vascular association of nanoparticles may also explain accumulation in lungs. Mesoporous and non-amine silica particles have been shown to accumulate in the lungs due to association with lung vascular cells instead of internalization by pulmonary cells (Yu et al., 2012). Furthermore, the particle surface may be gradually modified due to the hydrolysis of amide bonds which happens in-vivo (in liver) (Kumar et al., 2010). A bright fluorescence signal from lungs was observed at 6 hours (CDSIR820) and was still present at 48 hours in the animals administered free IR820 and CDSIR820. Kumar et al. confirmed that encapsulated dye can redirect the nanoparticles to its primary site of accumulation (Kumar et al., 2010). Another reason for lung accumulation might be due to some aggregation of our particles, as indicated by the DLS measurements. The bio distribution data suggested that after 24 hours, Ormosil nanoparticles are retained more in the spleen than the free dye.

It is important to note that even though Ormosil particles may not possess cytotoxic effects at low concentrations, vascular obstruction due to the formation of aggregates may pose a health hazard and hence strategies to further enhance particle stability and evade RES capture are needed. In a study by our group with IR820 PEG diamine particles, we found that free IR820 localized in liver and lungs after 24 hours, but IR820-PEG-diamine conjugates did not show any lung accumulation, presumably due to the presence of PEG. 


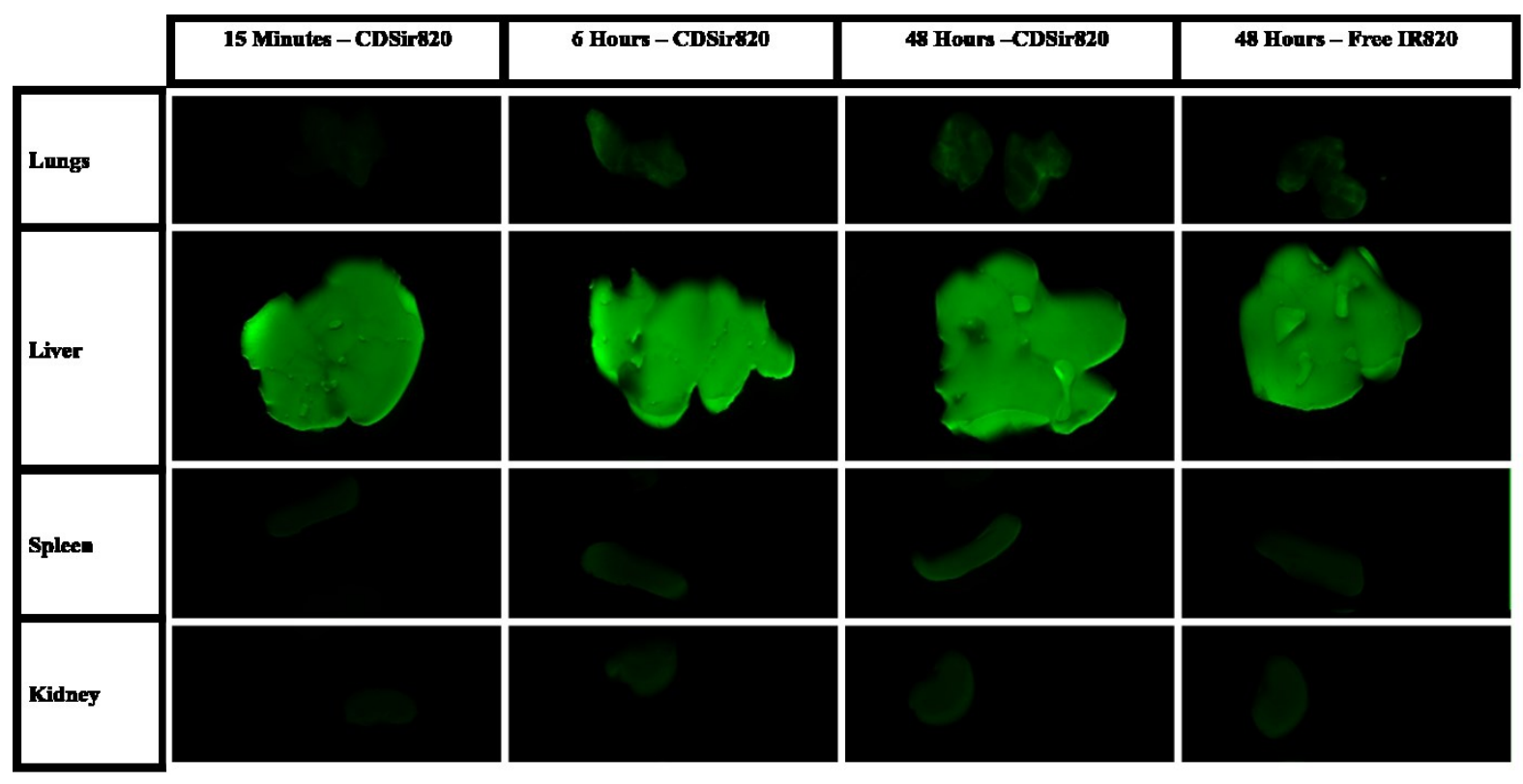

Figure 3.17: High NIR fluorescence is observed from the internal RES organs of mice. Fluorescence images of different organs at different time points obtained from LI-COR CLx imaging system.

The measured dye content was fit to a two-compartmental pharmacokinetics model to estimate the distribution and elimination half-life of IR820 encapsulated in PEGCDSIR820 particles. The distribution half-life $(\alpha 1 / 2)$ was estimated to be $11.4 \pm 4.2$ minutes and the elimination half-life $(\beta 1 / 2)$ was estimated as $41.2 \pm 7.1$ hours. The elimination half-life is significantly longer $(p<0.05)$ than free IR820, which was estimated to be $30.5 \pm 0.5$ hours from our previous studies (Fernandez-Fernandez et al., 2011). Recent reports from Qian et al. with IR820 loaded Ormosil particles showed sustained particle fluorescence at the tumor site until 30 days (Qian et al., 2012). 


\section{Plasma Clearence of PEGCDSIR820 Vs Time}

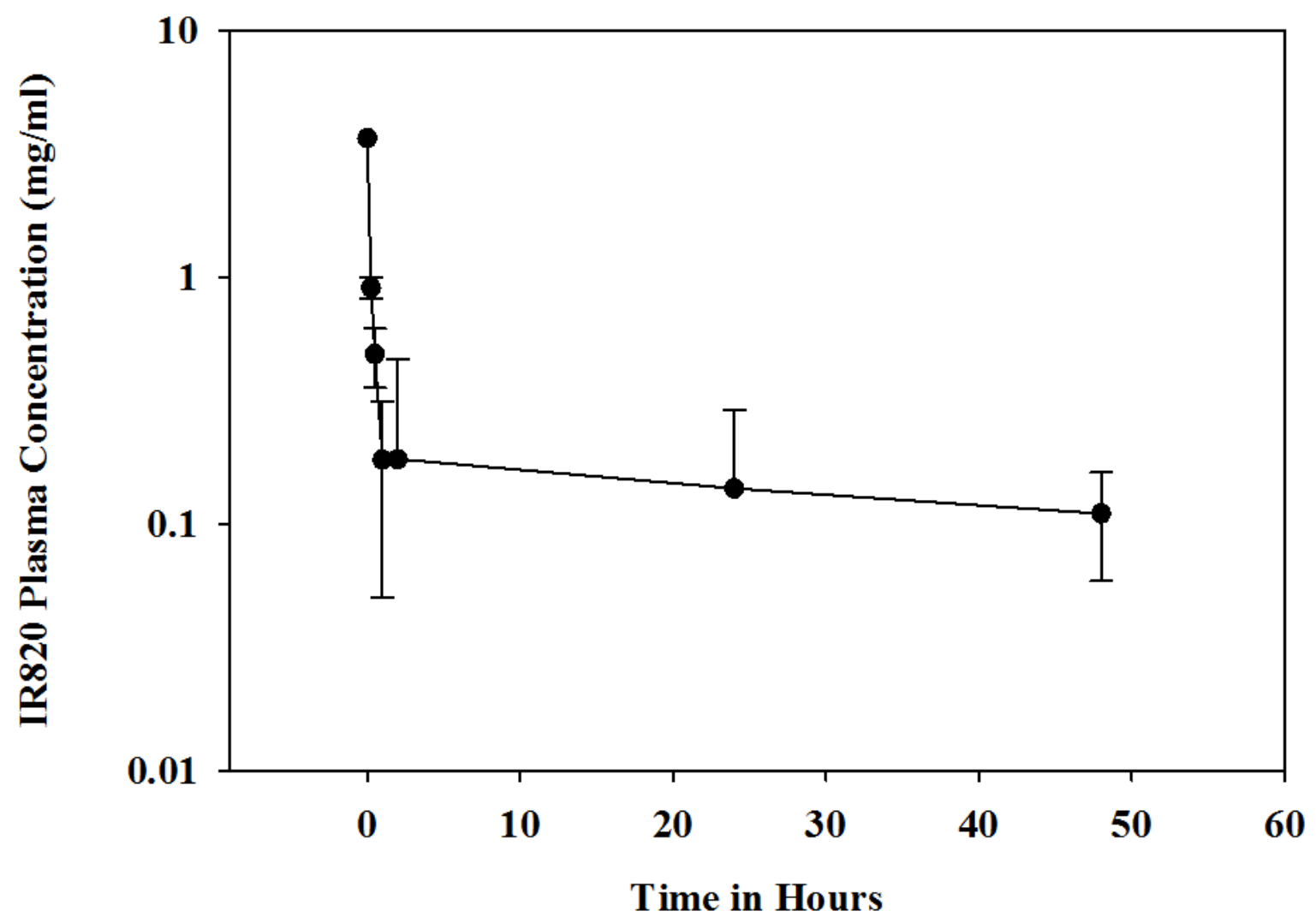

Figure 3.18 : Plasma pharmacokinetics show increased circulation time of IR820 administered using PEGCDSIR820 particles. Plasma clearance curves of IR820 vs Time after IV injection of PEGCDSIR820 particles. Y-Axis is shown in log units. Data is represented as Mean $\pm \mathrm{S}$.E of $n=3$.

The plasma concentration represents the nanoparticle concentration available to diffuse at the tumor periphery. This is represented by the following equation of two compartmental pharmacokinetics.

$$
N_{T}(t)=N_{o}\left(\alpha 2^{-t / \tau_{\alpha}}+\beta 2^{-t / \tau_{\beta}}\right)
$$


The parameters $\alpha(3.43), \beta(0.22)$ represent the fraction of pharmacokinetic phases and $\tau_{\alpha}$ (11.4 min)' $\tau_{\beta}$ (41.2 hours) represent the half-life in the respective phases. Equation (19) can be used as a time dependent boundary condition at the periphery of the spheroid instead of a constant boundary condition (of available nanoparticle concentration) that was used to model transport of nanoparticles under in-vitro incubation. Clearance of nanoparticles from plasma will reduce the available concentration to diffuse into the tumor. At the same time, the concentration gradient will also reach an equilibrium which may further slow-down the transport into the tumor (Figure 3.19). In the case below, the AUC of the tumor reduced to 0.18 compared to 0.38 in case of constant boundary condition for concentration. This represents a simple albeit realistic situation of nanoparticle administration in-vivo. Based on the results of simulations, hyperthermia delivery can be planned for the tumor. The simulations can be combined with in-vivo NIR fluorescence tracking for particles for hyperthermia planning upon tumor accumulations. However, to accurately predict the delivery under hyperthermia, the simulations will need to consider effects of convective transport on nanoparticle delivery, convection of heat due to blood flow, and the effect of hyperthermia on local blood pressure and interstitial tumor pressure to accurately predict the nanoparticle distribution in solid tumors. 


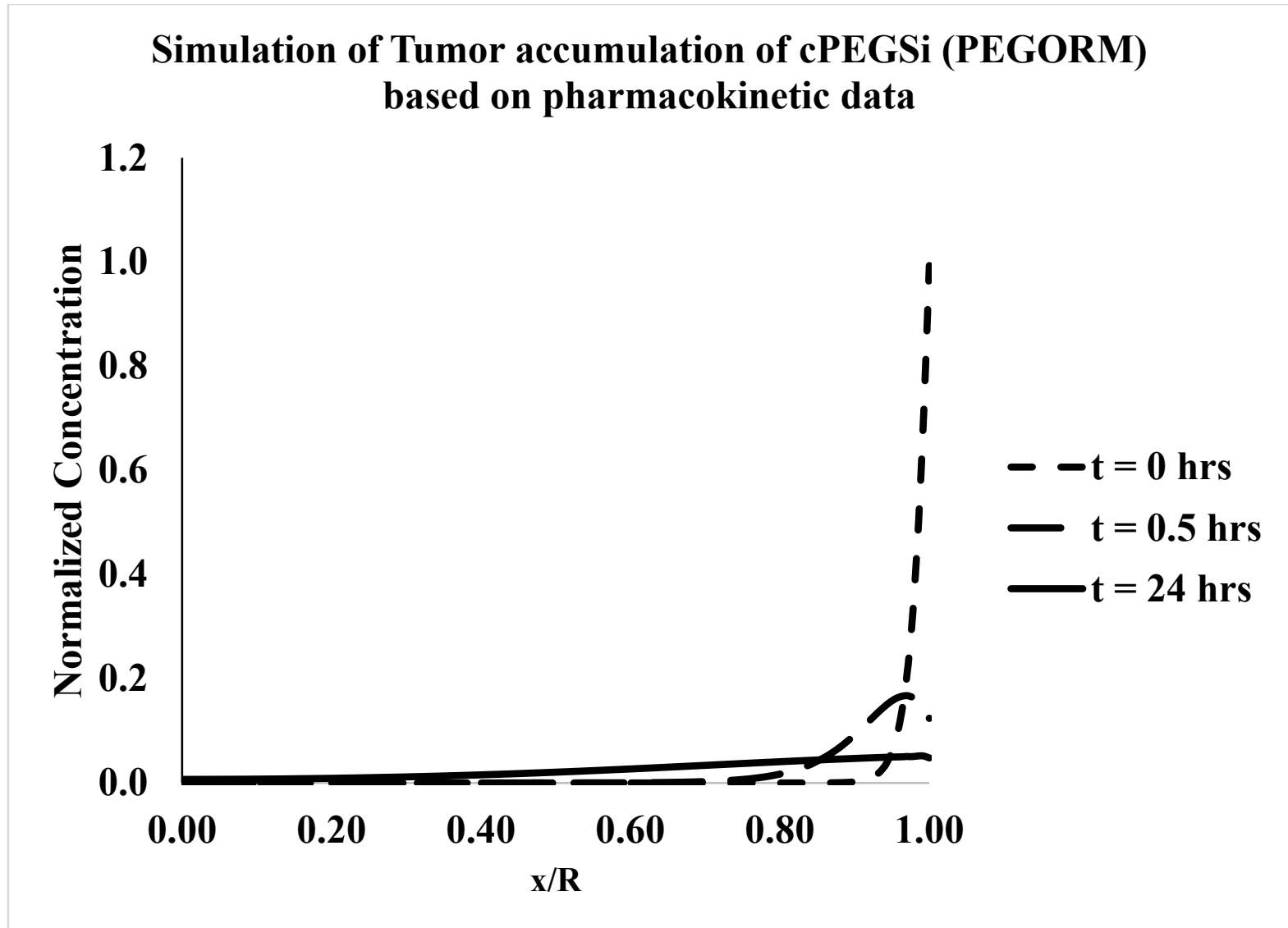

Figure 3.19 : Simulation of the effect of plasma clearance of nanoparticles on tumor accumulation.

DOX pharmacokinetics in-vivo was not measured, free DOX has been shown to have a similar elimination half-life as IR820, i.e., approximately 30 hours. Therefore, based on its slow release from the particles, it is reasonable to expect a similar increase in halflife of DOX encapsulated in PEGCDSIR820 as IR820. This expected increase of clearance half- life is in agreement with studies in the literature of the pharmacokinetics of a wellknown liposomal formulation of DOX, Doxil, that has been clinically approved by the FDA for treatment of ovarian cancer and AIDS related Kaposi Sarcoma (Gabizon et al., 2003). Other factor that may influence the biodistribution of DOX and IR820 in 
PEGCDSIR820 include the degradation of particles in-vivo, especially in the liver. This should be further investigated.

\subsection{Nanoparticle Design Criteria for Cancer Theranostics.}

Nanoparticle design for cancer theranostics should consider the physical and chemical properties of nanoparticles (viz size, surface charge, and other surface properties) in relation to the properties of tumor such as porosity, interstitial fluid pressure and cell uptake. As mentioned earlier, in solid tumors, the outward directed interstitial fluid pressure that arises due to the lack of lymphatic drainage and the poorly developed vasculature are primary hindrances to the convective transport of nanoparticles (Zhan et al., 2017). In such case, diffusion is the dominant transport mechanism for delivery of nanoparticles in solid tumors. A recent study by Sykes et al. has shown that tumor properties such as tumor volume and concurrently the ECM content and pore size determined gold nanoparticle penetration in MDA-MB-435 human breast adenocarcinoma mice xenografts (Sykes et al., 2016). As tumor volume increased, the porosity also increased as a result of loss in structural ECM via reduction in collagen fiber thickness (this was also observed for our large size MES-SA/Dx-5 aggregates that exhibited a highly porous structure). This led to an increase in sequestration of gold nanoparticles with diameter greater than $50 \mathrm{~nm}$ 's. In our simulations of highly porous tumors $(\varepsilon>=0.5)$, an increase in porosity (by cell death under hyperthermia) above the base threshold did not significantly increase the accumulation (i.e. AUC) of $50 \mathrm{~nm}$ silica nanoparticles. Therefore, in highly porous tumors, instead of promoting particle penetration, hyperthermia can be used to achieve other functions viz intracellular drug release or as an adjuvant once the 
drug has bound to its intracellular target. Moreover, particles with larger diameters ( $>50$ nm) maybe suitable for therapy of highly porous tumors since larger size particles can carry more payload compared to smaller size particles.

It may appear that decreasing the particle size below the pore threshold is a successful strategy to enhance the penetration of nanoparticles in tumors of low porosity. However, decreasing the particle size has the following disadvantages; 1) particles with diameter smaller than $10 \mathrm{~nm}$ are subject to rapid renal clearance, and 2) smaller particles have higher collision rate with the tumor ECM and expulsion rate from the tumor (Sykes et al., 2016). Experimental and mathematical simulations from literature studies have shown that nanoparticles in the size range 30-60 nm's offer a distinctive advantage over all other sizes in terms of cell uptake and tumor penetration.

As seen in chapter 2, the penetration of nanoparticles is also determined by the balance between physical (i.e. diffusive forces) and reaction forces (i.e. cell uptake). If the reaction force dominates the diffusive force, then nanoparticle transport into the tumors is impeded by the binding site barrier effect. Hence, it is essential to consider both when modeling nanoparticle transport in tumors. In the above case of PEGCDSIR820 transport in Skov-3 spheroids, the finite element solution predicted the $\mathrm{W}_{1 / 2}$ value for carboxy functionalized particles at 8 hours to be 0.80 . This implies that the nanoparticle concentration is more than the half maximal concentration (at the periphery) only till a 20 $\%$ distance from the spheroid surface. Due to the slow release of DOX from the particles, cells in the spheroid are not exposed to lethal concentration that will result in cell necrosis. Even though DOX release is enhanced under NIR hyperthermia, the application of NIR 
induced hyperthermia at 2 hours results in rapid aggregation of PEGORM which will impede nanoparticle penetration at further times. In conjunction with reduced uptake of nanoparticles by the cells, application of early NIR hyperthermia ( 2 hours) may not result in cell death. In 2 hours, the Ormosil particles only diffuse to $20 \%$ distance from the periphery. The distance increased to $50 \%$ at $24 \mathrm{hrs}$ and hence the adjuvant treatment was able to exert its cytotoxic effect on Skov-3 spheroids.

Furthermore, at the tumor cell, for the drug to reach its intracellular target (DNA in case of DOX), the nanoparticles should be up-taken by the cells where the drug should be released from the nanoparticle. Here, the release rate of drug from nanoparticles plays a critical role. While, fast release of drug from the nanoparticles reduces bioavailability of drugs due to rapid elimination of the leaked drug, slow/incomplete release also reduces the drug bioavailability in tumors. This was observed by Langinha et.al. for liposomal formulations of DOX administered (slow release) in mice tumor xenografts (Laginha et al., 2005). Only, $27 \%$ of encapsulated DOX from a fast releasing liposomal formulation could reach the cell DNA (27 \% bioavailability) and $50 \%$ of encapsulated DOX from DOXIL (slow release) could reach the intended target. The release rate should be tailored per the diffusion and uptake rate (penetration rate) of nanoparticles. When the penetration rate of nanoparticles is slow (in tumors with low porosity), the release rate should be slow so that the drug is retained by the carrier until it reaches its intracellular target in therapeutic doses. At the same time, very slow (extending over days or weeks) release of drugs may also reduce the bioavailability through reverse diffusion of nanoparticle carrier from the tumor and its subsequent clearance. Hence, it is desirable that all (preferably $100 \%$ ) of the drug 
be released from the nanoparticle during its residence time in the tumor. The goal of ondemand drug release (i.e. timing of laser exposure) on spheroid penetration and cellular internalization can be easily attained through the combination of on-demand stimuli responsive nanoparticles and mathematical modeling approach developed in this work. However, for PEGCDSIR820 particles it should be noted that though the adjuvant therapy shows increased efficacy in cell killing, incomplete release of drug from the particle is also its main limitation. Increasing the release further will be the goal of future studies.

\subsection{Conclusion.}

Novel theranostic Ormosil nanoparticles loaded with DOX and IR820 for chemotherapy, adjuvant hyperthermia and NIR imaging were developed using the ternary microemulsion method. Covalent loading of DOX on the silica particle surface slowed the release of DOX compared to physical adsorption, which resulted in rapid release of DOX from the particles (Figure 3.5). Pegylated nanoparticles possessed the physical properties, e.g., stability against aggregation in physiological media, size $(50-60 \mathrm{~nm})$ and surface charge $(-6.2 \mathrm{mV})$ for optimal cell uptake and passive targeting of tumors via the EPR effect (Table 3.1). Exposure to NIR laser resulted in a rapid rise of temperature to $43^{\circ} \mathrm{C}$ and $45 \%$ release of DOX from the pegylated nanoparticles in cell culture media in 3 hours (Figure $3.7 \&$ Figure 3.8). The comparison of cytotoxicity profiles between different loading modes show that FDSIR820 causes high toxicity in Skov-3 ovarian cancer cells whereas CDSIR820 particles are well tolerated by the cells (Figure 3.11). Pegylated particles without NIR exposure did not show any toxicity in Skov-3 cells up to a concentration of $360 \mu \mathrm{g} / \mathrm{ml}$, whereas non-pegylated particles showed toxicity in a dose dependent manner (Figure 3.11). 
The combination of DOX release and rapid rise hyperthermia resulted in enhanced cell killing of Skov-3 cells (Figure 3.11). Nanoparticle penetration and associated cytotoxicity were tested in Skov-3 spheroids grown in liquid overlay culture. The spheroids showed a very compact and dense aggregation of cells. DOX fluorescence from pegylated nanoparticles was in-homogeneously distributed around the periphery of Skov-3 spheroids at 8 hours. Exposure to laser increased DOX fluorescence into the interior of the spheroids but failed to elicit a measurable cytotoxic response at 8 hours (Figure 3.13). Exposure to NIR laser after 24 hours incubation with PEGCDSIR820 nanoparticles resulted in complete disaggregation of the spheroid mass as a result of cell death (Figure 3.16). Combined with cell uptake results that showed that a rapid rate NIR hyperthermia decreases cell uptake of nanoparticles (Figure 3.14), it can be concluded that the timing of adjuvant hyperthermia plays an important role to achieve a combined effect of both therapies. In-vivo biodistribution experiments showed that encapsulation increased the in-vivo circulation time of IR820 (Figure 3.18). PEGCDSIR820 particles can be further modified with cancer specific antibodies (HER2) or small molecules (folate) along-with PEG for selective targeting of ovarian cancer and other cancers as well. Furthermore, the spheroid model is a rigorous tool to study nanoparticle distribution. The results from spheroids can be readily translated to develop in-vivo strategies for combined chemotherapy and adjuvant hyperthermia.

\section{References}

1. Albanese, A.; Chan, W. C. Effect of gold nanoparticle aggregation on cell uptake and toxicity. ACS nano 2011, 5, 5478-5489. 
2. Arriagada, F.; Osseo-Asare, K. Synthesis of nanosize silica in a nonionic water-in-oil microemulsion: effects of the water/surfactant molar ratio and ammonia concentration. J. Colloid Interface Sci. 1999, 211, 210-220.

3. Bagwe, R. P.; Yang, C.; Hilliard, L. R.; Tan, W. Optimization of Dye-Doped Silica Nanoparticles Prepared Using a Reverse Microemulsion Method. Langmuir 2004, 20, 8336-8342.

4. Chatterjee, D. K.; Yong, Z. Upconverting nanoparticles as nanotransducers for photodynamic therapy in cancer cells. Nanomedicine 2008, 3, 73-82.

5. Cohn, D.; Sagiv, H.; Benyamin, A.; Lando, G. Engineering thermoresponsive polymeric nanoshells. Biomaterials 2009, 30, 3289-3296.

6. Comerford, K. M.; Wallace, T. J.; Karhausen, J.; Louis, N. A.; Montalto, M. C.; Colgan, S. P. Hypoxia-inducible Factor-1-dependent Regulation of the Multidrug Resistance (MDR1) Gene. Cancer Research 2002, 62, 3387-3394.

7. Dromi, S.; Frenkel, V.; Luk, A.; Traughber, B.; Angstadt, M.; Bur, M.; Poff, J.; Xie, J.; Libutti, S. K.; Li, K. C.; Wood, B. J. Pulsed-high intensity focused ultrasound and low temperature-sensitive liposomes for enhanced targeted drug delivery and antitumor effect. Clin. Cancer Res. 2007, 13, 2722-2727.

8. Ernsting, M. J.; Murakami, M.; Roy, A.; Li, S. Factors controlling the pharmacokinetics, biodistribution and intratumoral penetration of nanoparticles. $J$. Controlled Release 2013, 172, 782-794.

9. Fernandez-Fernandez, A.; Manchanda, R.; Lei, T.; Carvajal, D. A.; Tang, Y.; Raza Kazmi, S. Z.; McGoron, A. J. Comparative study of the optical and heat generation properties of IR820 and indocyanine green. Molecular imaging 2012, 11, 99.

10. Fernandez-Fernandez, A.; Manchanda, R.; Tingjun, L.; Tang, Y.; Carvajal, D. A.; McGoron, A. J. IR820-PEG-diamine nanocomplexes for image-guided hyperthermia. 2011.

11. Friedman, R. Nano dot technology enters clinical trials. J. Natl. Cancer Inst. 2011, 103, 1428-1429.

12. Gabizon, A.; Shmeeda, H.; Barenholz, Y. Pharmacokinetics of pegylated liposomal doxorubicin. Clin. Pharmacokinet. 2003, 42, 419-436.

13. Gao, X.; Cui, Y.; Levenson, R. M.; Chung, L. W.; Nie, S. In vivo cancer targeting and imaging with semiconductor quantum dots. Nat. Biotechnol. 2004, 22, 969-976. 
14. Goodman, T. T.; Olive, P. L.; Pun, S. H. Increased nanoparticle penetration in collagenase-treated multicellular spheroids. International journal of nanomedicine 2007, 2, 265.

15. Hartlen, K. D.; Athanasopoulos, A. P.; Kitaev, V. Facile preparation of highly monodisperse small silica spheres $(15 \mathrm{to}>200 \mathrm{~nm})$ suitable for colloidal templating and formation of ordered arrays. Langmuir 2008, 24, 1714-1720.

16. Huang, X.; Jain, P. K.; El-Sayed, I. H.; El-Sayed, M. A. Plasmonic photothermal therapy (PPTT) using gold nanoparticles. Lasers in medical science 2008, 23, 217 228.

17. Jain, P. K.; Lee, K. S.; El-Sayed, I. H.; El-Sayed, M. A. Calculated absorption and scattering properties of gold nanoparticles of different size, shape, and composition: applications in biological imaging and biomedicine. The Journal of Physical Chemistry B 2006, 110, 7238-7248.

18. Jain, R. K. Transport of molecules, particles, and cells in solid tumors. Annu. Rev. Biomed. Eng. 1999, 1, 241-263.

19. Jain, R. K.; Stylianopoulos, T. Delivering nanomedicine to solid tumors. Nature reviews clinical oncology 2010, 7, 653-664.

20. Kumar, P.; Roy, I. Optically and magnetically doped ormosil nanoparticles for bioimaging: synthesis, characterization, and in vitro studies. RSC Advances 2014, 4, 16181-16187.

21. Kumar, R.; Roy, I.; Ohulchanskky, T. Y.; Vathy, L. A.; Bergey, E. J.; Sajjad, M.; Prasad, P. N. In vivo biodistribution and clearance studies using multimodal organically modified silica nanoparticles. ACS nano 2010, 4, 699-708.

22. Laginha, K. M.; Verwoert, S.; Charrois, G. J.; Allen, T. M. Determination of doxorubicin levels in whole tumor and tumor nuclei in murine breast cancer tumors. Clin. Cancer Res. 2005, 11, 6944-6949.

23. Law, W.; Yong, K.; Roy, I.; Xu, G.; Ding, H.; Bergey, E. J.; Zeng, H.; Prasad, P. N. Optically and magnetically doped organically modified silica nanoparticles as efficient magnetically guided biomarkers for two-photon imaging of live cancer cells $\dagger$. The Journal of Physical Chemistry C 2008, 112, 7972-7977.

24. Lee, E. S.; Gao, Z.; Bae, Y. H. Recent progress in tumor pH targeting nanotechnology. J. Controlled Release 2008, 132, 164-170.

25. Lee, J. M.; Mhawech-Fauceglia, P.; Lee, N.; Parsanian, L. C.; Lin, Y. G.; Gayther, S. A.; Lawrenson, K. A three-dimensional microenvironment alters protein expression 
and chemosensitivity of epithelial ovarian cancer cells in vitro. Laboratory investigation 2013, 93, 528-542.

26. Lei, T.; Manchanda, R.; Fernandez-Fernandez, A.; Huang, Y.; Wright, D.; McGoron, A. J. Thermal and $\mathrm{pH}$ sensitive multifunctional polymer nanoparticles for cancer imaging and therapy. RSC advances 2014, 4, 17959-17968.

27. Lepkowicz, R. S.; Cirloganu, C. M.; Przhonska, O. V.; Hagan, D. J.; Van Stryland, E. W.; Bondar, M. V.; Slominsky, Y. L.; Kachkovski, A. D.; Mayboroda, E. I. Absorption anisotropy studies of polymethine dyes. Chem. Phys. 2004, 306, 171183.

28. Lesniak, A.; Campbell, A.; Monopoli, M. P.; Lynch, I.; Salvati, A.; Dawson, K. A. Serum heat inactivation affects protein corona composition and nanoparticle uptake. Biomaterials 2010, 31, 9511-9518.

29. Lesniak, A.; Fenaroli, F.; Monopoli, M. P.; Åberg, C.; Dawson, K. A.; Salvati, A. Effects of the presence or absence of a protein corona on silica nanoparticle uptake and impact on cells. ACS nano 2012, 6, 5845-5857.

30. Li, M.; Keller, P. Stimuli-responsive polymer vesicles. Soft Matter 2009, 5, 927-937.

31. Li, S.; Ma, Y.; Yue, X.; Cao, Z.; Dai, Z. One-pot construction of doxorubicin conjugated magnetic silica nanoparticles. New Journal of Chemistry 2009, 33, 24142418 .

32. Manchanda, R.; Fernandez-Fernandez, A.; Nagesetti, A.; McGoron, A. J. Preparation and characterization of a polymeric (PLGA) nanoparticulate drug delivery system with simultaneous incorporation of chemotherapeutic and thermo-optical agents. Colloids and Surfaces B: Biointerfaces 2010, 75, 260-267.

33. Moret, F.; Selvestrel, F.; Lubian, E.; Mognato, M.; Celotti, L.; Mancin, F.; Reddi, E. PEGylation of ORMOSIL nanoparticles differently modulates the in vitro toxicity toward human lung cells. Arch. Toxicol. 2015a, 89, 607-620.

34. Moret, F.; Selvestrel, F.; Lubian, E.; Mognato, M.; Celotti, L.; Mancin, F.; Reddi, E. PEGylation of ORMOSIL nanoparticles differently modulates the in vitro toxicity toward human lung cells. Arch. Toxicol. 2015b, 89, 607-620.

35. Motornov, M.; Roiter, Y.; Tokarev, I.; Minko, S. Stimuli-responsive nanoparticles, nanogels and capsules for integrated multifunctional intelligent systems. Progress in Polymer Science 2010, 35, 174-211.

36. Nakamura, M. Biomedical applications of organosilica nanoparticles toward theranostics. Nanotechnology Reviews 2012, 1, 469-491. 
37. Olejniczak, Z.; Łęczka, M.; Cholewa-Kowalska, K.; Wojtach, K.; Rokita, M.; Mozgawa, W. 29 Si MAS NMR and FTIR study of inorganic-organic hybrid gels. $J$. Mol. Struct. 2005, 744, 465-471.

38. Park, J.; Gu, L.; Von Maltzahn, G.; Ruoslahti, E.; Bhatia, S. N.; Sailor, M. J. Biodegradable luminescent porous silicon nanoparticles for in vivo applications. Nature materials 2009, 8, 331-336.

39. Przhonska, O. V.; Hu, H.; Webster, S.; Bricks, J. L.; Viniychuk, A. A.; Kachkovski, A. D.; Slominsky, Y. L. Electronic transitions in a series of 2-azaazulene polymethine dyes with different $\pi$-conjugation lengths. Chem. Phys. 2013, 411, 1725 .

40. Qian, J.; Wang, D.; Cai, F.; Zhan, Q.; Wang, Y.; He, S. Photosensitizer encapsulated organically modified silica nanoparticles for direct two-photon photodynamic therapy and in vivo functional imaging. Biomaterials 2012, 33, 4851-4860.

41. Raghunand, N.; Mahoney, B. P.; Gillies, R. J. Tumor acidity, ion trapping and chemotherapeutics: II. pH-dependent partition coefficients predict importance of ion trapping on pharmacokinetics of weakly basic chemotherapeutic agents. Biochem.

Pharmacol. 2003, 66, 1219-1229.

42. Roy, I.; Kumar, P.; Kumar, R.; Ohulchanskyy, T. Y.; Yong, K.; Prasad, P. N. Ormosil nanoparticles as a sustained-release drug delivery vehicle. RSC Advances 2014, 4, 53498-53504.

43. Roy, I.; Ohulchanskyy, T. Y.; Pudavar, H. E.; Bergey, E. J.; Oseroff, A. R.; Morgan, J.; Dougherty, T. J.; Prasad, P. N. Ceramic-based nanoparticles entrapping waterinsoluble photosensitizing anticancer drugs: a novel drug-carrier system for photodynamic therapy. J. Am. Chem. Soc. 2003, 125, 7860-7865.

44. Salvati, A.; Åberg, C.; dos Santos, T.; Varela, J.; Pinto, P.; Lynch, I.; Dawson, K. A. Experimental and theoretical comparison of intracellular import of polymeric nanoparticles and small molecules: toward models of uptake kinetics. Nanomedicine: Nanotechnology, Biology and Medicine 2011, 7, 818-826.

45. Saxena, V.; Sadoqi, M.; Shao, J. Enhanced photo-stability, thermal-stability and aqueous-stability of indocyanine green in polymeric nanoparticulate systems. Journal of Photochemistry and Photobiology B: Biology 2004, 74, 29-38.

46. Selvestrel, F.; Moret, F.; Segat, D.; Woodhams, J. H.; Fracasso, G.; Echevarria, I. M. R.; Baù, L.; Rastrelli, F.; Compagnin, C.; Reddi, E. Targeted delivery of photosensitizers: efficacy and selectivity issues revealed by multifunctional ORMOSIL nanovectors in cellular systems. Nanoscale 2013a, 5, 6106-6116. 
47. Selvestrel, F.; Moret, F.; Segat, D.; Woodhams, J. H.; Fracasso, G.; Echevarria, I. M. R.; Baù, L.; Rastrelli, F.; Compagnin, C.; Reddi, E. Targeted delivery of photosensitizers: efficacy and selectivity issues revealed by multifunctional ORMOSIL nanovectors in cellular systems. Nanoscale 2013b, 5, 6106-6116.

48. Shan, L. In Protoporphyrin IX and IR-820 fluorophore-encapsulated organically modified silica nanoparticles; Molecular Imaging and Contrast Agent Database (MICAD); Bethesda (MD), 2004; .

49. Shapero, K.; Fenaroli, F.; Lynch, I.; Cottell, D. C.; Salvati, A.; Dawson, K. A. Time and space resolved uptake study of silica nanoparticles by human cells. Mol. BioSyst. 2011, 7, 371-378.

50. Slowing, I. I.; Vivero-Escoto, J. L.; Wu, C.; Lin, V. S. Mesoporous silica nanoparticles as controlled release drug delivery and gene transfection carriers. $A d v$. Drug Deliv. Rev. 2008, 60, 1278-1288.

51. Srinivasan, S.; Bhardwaj, V.; Nagasetti, A.; Fernandez-Fernandez, A.; McGoron, A. J. Multifunctional Surface-Enhanced Raman Spectroscopy-Detectable Silver Nanoparticles for Combined Photodynamic Therapy and $\mathrm{pH}$-Triggered Chemotherapy. Journal of Biomedical Nanotechnology 2016, 12, 2202-2219.

52. Stöber, W.; Fink, A.; Bohn, E. Controlled growth of monodisperse silica spheres in the micron size range. J. Colloid Interface Sci. 1968, 26, 62-69.

53. Sykes, E. A.; Dai, Q.; Sarsons, C. D.; Chen, J.; Rocheleau, J. V.; Hwang, D. M.; Zheng, G.; Cramb, D. T.; Rinker, K. D.; Chan, W. C. Tailoring nanoparticle designs to target cancer based on tumor pathophysiology. Proc. Natl. Acad. Sci. U. S. A. 2016, 113, E1142-51.

54. Tai, L.; Tsai, P.; Wang, Y.; Wang, Y.; Lo, L.; Yang, C. Thermosensitive liposomes entrapping iron oxide nanoparticles for controllable drug release. Nanotechnology 2009, 20, 135101.

55. Tang, Y. Cancer Therapy Combining Modalities of Hyperthermia and Chemotherapy: in vitro Cellular Response after Rapid Heat Accumulation in the Cancer Cell, 2010.

56. Tang, Y.; McGoron, A. J. Combined effects of laser-ICG photothermotherapy and doxorubicin chemotherapy on ovarian cancer cells. Journal of Photochemistry and Photobiology B: Biology 2009, 97, 138-144.

57. Tenzer, S.; Docter, D.; Kuharev, J.; Musyanovych, A.; Fetz, V.; Hecht, R.; Schlenk, F.; Fischer, D.; Kiouptsi, K.; Reinhardt, C. Rapid formation of plasma protein corona critically affects nanoparticle pathophysiology. Nature nanotechnology 2013, $8,772-781$. 
58. Timko, B. P.; Dvir, T.; Kohane, D. S. Remotely triggerable drug delivery systems. Adv Mater 2010, 22, 4925-4943.

59. von Maltzahn, G.; Centrone, A.; Park, J.; Ramanathan, R.; Sailor, M. J.; Hatton, T. A.; Bhatia, S. N. SERS-coded gold nanorods as a multifunctional platform for densely multiplexed near-infrared imaging and photothermal heating. Adv Mater 2009, 21, 3175-3180.

60. Wong, C.; Stylianopoulos, T.; Cui, J.; Martin, J.; Chauhan, V. P.; Jiang, W.; Popovic, Z.; Jain, R. K.; Bawendi, M. G.; Fukumura, D. Multistage nanoparticle delivery system for deep penetration into tumor tissue. Proc. Natl. Acad. Sci. U. S. A. 2011, 108, 2426-2431.

61. Wust, >. P.; Gneveckow, U.; Wust, >. P.; Gneveckow, U.; Johannsen, M.; Böhmer, D.; Henkel, T.; Kahmann, F.; Sehouli, J.; Felix, R. Magnetic nanoparticles for interstitial thermotherapy-feasibility, tolerance and achieved temperatures. International Journal of Hyperthermia 2006, 22, 673-685.

62. Yi, X.; Wang, F.; Qin, W.; Yang, X.; Yuan, J. Near-infrared fluorescent probes in cancer imaging and therapy: an emerging field. Int. J. Nanomedicine 2014, 9, 13471365 .

63. You, J.; Zhang, G.; Li, C. Exceptionally high payload of doxorubicin in hollow gold nanospheres for near-infrared light-triggered drug release. ACS nano 2010, 4, 1033 1041 .

64. Yu, M. K.; Jeong, Y. Y.; Park, J.; Park, S.; Kim, J. W.; Min, J. J.; Kim, K.; Jon, S. Drug-loaded superparamagnetic iron oxide nanoparticles for combined cancer imaging and therapy in vivo. Angewandte Chemie International Edition 2008, 47, 5362-5365.

65. Yu, T.; Hubbard, D.; Ray, A.; Ghandehari, H. In vivo biodistribution and pharmacokinetics of silica nanoparticles as a function of geometry, porosity and surface characteristics. J. Controlled Release 2012, 163, 46-54.

66. Zhan, W.; Gedroyc, W.; Xu, X. Y. The effect of tumour size on drug transport and uptake in 3-D tumour models reconstructed from magnetic resonance images. PloS one 2017, 12, e0172276.

67. Zhang, S.; Li, J.; Lykotrafitis, G.; Bao, G.; Suresh, S. Size-Dependent Endocytosis of Nanoparticles. Adv Mater 2009, 21, 419-424. 


\section{Final Conclusions and Future Work}

The tumor microenvironment is a critical determinant of response to therapy. The poor penetration of nutrients and oxygen due to the presence of extracellular matrix in tumors creates regions of hypoxia and low $\mathrm{pH}$ that alter the response of cells to drugs. Currently, monolayer cultures of cells cannot recreate the structural and functional complexity of in-vivo tumors. Multicellular tumor spheroids recreate the morphological and functional characteristics of in-vivo avascular/solid tumors. In this study, 3dimensional tumor models were generated from ovarian carcinoma (Skov-3) and uterine sarcoma cells (MES-SA/Dx5). Skov-3 spheroids showed compact organization that was also verified by scanning electron microscopy (SEM) whereas, MES-SA/Dx5 resembled cell aggregates with distinctly visible cells under SEM. Both models (Skov3 and MESSA/Dx5) were resistant to DOX and adjuvant hyperthermia, probably owing to the drug and thermal tolerance that are enhanced due to the three dimensional organization of tumor spheroids. These results are in contrast to those obtained in monolayers which showed enhancement in cell killing from the combination of DOX and hyperthermia. For a successful treatment, the mechanisms of drug and thermal resistance originating due to the three-dimensional organization of tumor spheroids need to be circumvented.

Nanoparticles have emerged as a logical choice to specifically deliver encapsulated agents to tumors safely, without the systemic toxicity of free drugs. The physical (nanodimensions) and chemical properties (surface and nanomaterial properties) of nanoparticles are advantageous to specifically deliver the encapsulated agents to the 
tumors, either via passive targeting through the EPR effect or by specific targeting of cancer cell receptors. At the same time, drug delivery using nanoparticles has been shown to circumvent P-gp mediated drug tolerance. However, owing to a bigger size compared to free drug moieties, nanoparticles suffer from poor penetration into avascular/solid tumors. Several factors such as the size, surface charge of nanoparticles, binding with cells in spheroids and stability of nanoparticle against aggregation determine the penetration of nanoparticles into tumor spheroids. Hyperthermia can also influence nanoparticle delivery to spheroids by 1) affecting the colloidal stability of nanoparticles, 2) altering the uptake of nanoparticles by the cells and 3) changing the porosity of spheroids due to thermal damage related cell death. At present there are no reported methods to estimate the kinetics of nanoparticle uptake by cells under hyperthermia. A novel method of inverse estimation based on Bayesian statistics was developed to fit the experimental measurements of fluorescent silica nanoparticle uptake by the Skov-3 cells. The solution to the inverse problem was found using the Markov Chain Monte Carlo technique. The method was able to successfully predict all four rate parameters of cell uptake i.e., association rate $\left(\mathrm{k}_{\mathrm{a}}\right)$, dissociation rate $\left(\mathrm{k}_{\mathrm{r}}\right)$, internalization rate $\left(\mathrm{k}_{\mathrm{i}}\right)$ and externalization rate $\left(\mathrm{k}_{\mathrm{rec}}\right)$ from a wide parameter space, independent of initial guess. Under hyperthermia, the kinetics of particle association and internalization increased compared to $37^{\circ} \mathrm{C}$. However, hyperthermia did not cause a significant increase in amount of nanoparticles taken up by the cells compared to $37^{\circ} \mathrm{C}$. The experiments also showed the importance of surface modification on nanoparticle uptake, nanoparticles modified with inert polyethylene glycol (methoxy-PEG) were taken up less by the cells compared to carboxy-PEG modified silica nanoparticles. The parameters predicted from MCMC were used in an advection reaction model that was 
coupled with the Arrhenius model of thermal damage to simulate the effect of hyperthermia on nanoparticle transport in tumor spheroids. Application of hyperthermia increased the effective size of particles through aggregation and also increased tumor porosity due to thermal damage related cell death. The transport of silica nanoparticles was diffusion dominated and an increase in porosity lead to an increase in penetration (distance) of nanoparticles. The inverse estimation approach combined with the finite element model can be used to predict optimal set of nanoparticle properties (for a given condition; hyperthermia) such as size, surface charge, cellular uptake parameters that are suitable for deep penetration into the avascular tumor. Based on these observations, drug loading and the density of moieties on the nanoparticle surface can be designed. Additionally, in case of hyperthermia, based on the tumor characteristics, the thermal dose necessary to cause a desired increase in porosity for deep penetration of nanoparticles can also be determined.

Based on the observations of nanoparticle transport in tumor spheroids and response to DOX under hyperthermia, a novel multifunctional theranostic probe for rapid rise hyperthermia was developed for image guided chemotherapy with adjuvant hyperthermia. Rapid rise hyperthermia is beneficial to prevent thermal tolerance (HSP70 expression) associated with slow hyperthermia. Organically modified silica nanoparticles were encapsulated with a near infrared dye (NIR; IR820) and Doxorubicin (DOX). Ormosil nanoparticles were formulated using the ternary microemulsion method and their surface was modified with Polyethylene Glycol (PEG). The size, surface properties and colloidal stability in physiological media of the pegylated particles are ideal for passive targeting of the tumors via Enhanced Permeation and Retention Effect (EPR). DOX was loaded into 
the nanoparticles via physical adsorption or by chemical conjugation. IR820 showed strong interaction with the Ormosil matrix as determined by a shift in the absorbance spectra and was therefore retained effectively by the Ormosil nanoparticles in physiological buffers. Owing to its hydrophobic nature, DOX was effectively retained by the nanoparticles in aqueous buffers. However, physical adsorption of DOX lead to rapid leakage by partitioning the hydrophobic components of the media. Covalent conjugation of DOX led to a very slow release due to slow hydrolysis of urea linkage between DOX and silica matrix. Pegylated Ormosil particles generated hyperthermia, rapidly, multiple times on successive exposures to NIR laser. Exposure to laser also led to an increase in release of DOX from the particles. These factors (heat and DOX release) led to enhanced cell killing by PEGylated Ormosil particles which were otherwise non-toxic to Skov-3 cells in the absence of laser exposure. In spheroids, exposure to hyperthermia led to deeper penetration of DOX, however, early application of laser exposure before the particles were able to penetrate the periphery of the spheroids did not cause any increase in the toxicity. This was possibly due to extensive aggregation observed for PEGylated Ormosil particles on laser exposure which impeded nanoparticle transport into the spheroid. Laser exposure of Skov3 spheroids after 24 hours of incubation with PEGylated Ormosil nanoparticles resulted in the marked destruction of spheroid structure due to cell death. By properly planning the hyperthermia treatment maximum therapeutic efficacy was achieved in killing tumor spheroids. This efficacy was not achieved with slow rate hyperthermia. From these results it is clear that for both cases i.e. free DOX therapy and nanoparticle therapy, successive applications of DOX plus hyperthermia may not be beneficial to achieve an additive effect in avascular tumors. In-vivo experiments in mice showed that encapsulation of IR820 
increased the elimination half-life compared to its free form. The bio-distribution profile of Ormosil nanoparticles showed uptake by the organs of the RES. The vascular association and presence of particles in the lungs remains a concern for successful application of these particles for therapy in-vivo.

Future Work: The tumor spheroids in this work were generated from a single cell type, as noted earlier, in-vivo tumor tissues consist of various cell types that ultimately determine the tumor organization and response to therapy. Additionally, dynamic conditions that simulate flow and pressure characteristics in tumors are also needed to accurately model nanoparticle transport. Hence, co-culture of different cell types into spheroids and incorporation of spheroids with microfluidic chambers that simulate flow patterns in a tumor will create a suitable 'tumor on chip' platform for drug testing. The inverse estimation method for estimating rate parameters of cellular uptake of nanoparticles can be coupled with experiments with different nanoparticles (gold, iron, silver, etc.) cell types and environmental conditions (low $\mathrm{pH}$ and hypoxia) to generate appropriate constraints to accurately 'train' the method to predict the physical phenomenon of uptake. In order to further increase the accuracy of finite element predictions of nanoparticle transport, detailed information regarding tumor characteristics will be needed. Other modeling techniques such as, Monte Carlo, meshless, particle tracking and Lattice Boltzmann methods may also be developed to overcome limitations of the finite element method.

Presently, the theranostic Ormosil probes are ideal for passive targeting to the tumor. In the future, along-with PEG to evade RES clearance, the surface of these probes can also be 
modified with ligands specific to cancer cell receptors (example: folic acid, anti-HER2) for active targeting of the tumors. In-vivo studies in animal tumor models are needed to evaluate the efficacy of Ormosil mediated adjuvant NIR hyperthermia and Doxorubicin therapy on tumor reduction or eradication. Additionally, the slow release of DOX is a limitation to improving the therapeutic efficacy of DOX loaded Ormosil particles. Other strategies such as mesoporous silica particles and $\mathrm{pH}$ or photo cleavable bonds should be tried to enhance the DOX release in response to tumor microenvironment or external stimuli. The DOX circulation and release of DOX due to particle degradation when they are metabolized also needs to be investigated. 


\section{Appendix A: List of model parameters.}

\begin{tabular}{|l|l|l|}
\hline Parameter & Description & Units (SI) \\
\hline $\mathrm{r}$ & radial coordinate & $\mu \mathrm{m}$ \\
\hline $\mathrm{t}$ & time & $\mathrm{s}$ \\
\hline $\mathrm{C}$ & Interstitial concentration of nanoparticles in the spheroid & $\mathrm{M}$ \\
\hline$\varepsilon$ & Porosity of the spheroid & dimensionless \\
\hline $\mathrm{C}_{\mathrm{bs}}$ & Available binding sites of the cells in the spheroid & $\mathrm{M}$ \\
\hline $\mathrm{C}_{\mathrm{b}}$ & Bound concentration of nanoparticles to the cells & $\mathrm{M}$ \\
\hline $\mathrm{k}_{\mathrm{a}}$ & Association rate of nanoparticles with the cells & $\mathrm{M}^{-1} \mathrm{~s}^{-1}$ \\
\hline $\mathrm{k}_{\mathrm{d}}$ & Dissociation rate of nanoparticles from the cells & $\mathrm{s}^{-1}$ \\
\hline $\mathrm{k}_{\mathrm{i}}$ & Internalization rate of nanoparticles by the cells & $\mathrm{s}^{-1}$ \\
\hline $\mathrm{k}_{\mathrm{rec}}$ & Externalization rate of nanoparticles from the cells & $\mathrm{s}^{-1}$ \\
\hline $\mathrm{D}_{\mathrm{eff}}$ & Effective diffusion coefficient & $\mathrm{m} / \mathrm{s}$ \\
\hline $\mathrm{D}_{0}$ & Ideal diffusion coefficient & $\mathrm{m} / \mathrm{s}$ \\
\hline$\tau$ & Tortuosity & $\mathrm{Dimensionless}$ \\
\hline $\mathrm{R}$ & Spheroid Radius & $\mathrm{m}$ \\
\hline $\mathrm{B}_{\mathrm{max}}$ & Cell Surface Binding Capacity of Spheroids & $\mathrm{mol} / \mathrm{L}$ \\
\hline $\mathrm{C}_{\mathrm{Medium}}$ & External Boundary Condition & $\mathrm{mol} / \mathrm{L}$ \\
\hline $\mathrm{k}_{\mathrm{B}}$ & Boltzmann Constant & $\left(\mathrm{m}^{\wedge} 2 * \mathrm{~kg}\right) /\left(\mathrm{s}^{\wedge} 2 * \mathrm{~K}\right)$ \\
\hline $\mathrm{muCp}$ & Viscosity of Fluid (media) & $\mathrm{Pa} \mathrm{s}$ \\
\hline $\mathrm{a}$ & Particle Radius & $\mathrm{nm}$ \\
\hline $\mathrm{r}_{\mathrm{p}}$ & Collagen Fiber Radius & $\mathrm{m}$ \\
\hline$\lambda$ & Ratio of Nanoparticle Radius to Pore Size & $\mathrm{dimensionless}$ \\
\hline $\mathrm{Ax}$ & Activation Energy & $\mathrm{s}^{-1}$ \\
\hline $\mathrm{EAx}$ & Activation Energy Barrier & $\mathrm{J}^{*} \mathrm{~mol}{ }^{-1}$ \\
\hline $\mathrm{R}$ & Universal Gas Constant & $\mathrm{J}^{*}\left(\mathrm{~mol}^{-1 *} \mathrm{~K}^{-1}\right)$ \\
\hline
\end{tabular}


VITA

\section{ABHIGNYAN NAGESETTI}

Born, Hyderabad, India

2001-2005

B.Tech, Biomedical Engineering

Jawaharlal Technological University,

Hyderabad,India.

2012-2016

Graduate Assistant

Office of Global Learning Initiatives

Florida International University

Miami, FL,USA.

2008-2017

Ph.D Biomedical Engineering

Florida International University,

Miami, FL,USA

\section{PUBLICATIONS AND PRESENTATIONS}

Nagesetti, A.; McGoron, A. J. Multifunctional organically modified silica nanoparticles for chemotherapy, adjuvant hyperthermia and near infrared imaging. Colloids and Surfaces B: Biointerfaces 2016, 147, 492-500.

Nagesetti, A., Rodzinski, A., Stimphil, E., Khanal, T. S. C., Wang, P., Guduru, R., . . .Khizroev, S. (2017). Multiferroic coreshell magnetoelectric nanoparticles as NMR sensitive nanoprobes for cancer cell detection. Scientific Reports, 7(1), 1610-017- 01647$\mathrm{x}$.

Stimphil, E., Nagesetti, A., Guduru, R., Stewart, T., Rodzinski, A., Liang, P., \&amp; Khizroev, S. (2017). Physics considerations in targeted anticancer drug delivery by magnetoelectric nanoparticles. Applied Physics Reviews, 4(2), 021101

Srinivasan, S.; Bhardwaj, V.; Nagesetti, A.; Fernandez-Fernandez, A.; McGoron, A. J. Multifunctional Surface-Enhanced Raman Spectroscopy-Detectable Silver Nanoparticles for Combined Photodynamic Therapy and pH-Triggered Chemotherapy. Journal of Biomedical Nanotechnology 2016, 12, 2202-2219.

Srinivasan, S.; Manchanda, R.; Lei, T.; Nagesetti, A.; Fernandez-Fernandez, A.; McGoron, A. J. Targeted nanoparticles for simultaneous delivery of chemotherapeutic and hyperthermia agents-an in vitro study. Journal of Photochemistry and Photobiology B: Biology 2014, 136, 81-90.

Lei, T.; Srinivasan, S.; Tang, Y.; Manchanda, R.; Nagesetti, A.; Fernandez-Fernandez, A.; McGoron, A. J. Comparing cellular uptake and cytotoxicity of targeted drug carriers 
in cancer cell lines with different drug resistance mechanisms. Nanomedicine:

Nanotechnology, Biology and Medicine 2011, 7, 324-332.

Manchanda, R.; Fernandez-Fernandez, A.; Nagesetti, A.; McGoron, A. J. Preparation and characterization of a polymeric (PLGA) nanoparticulate drug delivery system with simultaneous incorporation of chemotherapeutic and thermo-optical agents. Colloids and Surfaces B: Biointerfaces 2010, 75, 260-267.

Tang, Y.; Lei, T.; Manchanda, R.; Nagesetti, A.; Fernandez-Fernandez, A.; Srinivasan, S.; McGoron, A. Simultaneous Delivery of Chemotherapeutic and Thermal-Optical Agents to Cancer Cells by a Polymeric (PLGA) Nanocarrier: An In Vitro Study. Pharm. Res. 2010, 27, 2242-2253.

Abhignyan Nagesetti, George Dulikravich \& Anthony McGoron: Multifunctional Organically Modified Silica Nanoparticles for Image Guided Chemotherapy with Ondemand Drug Release and Deep Tumor Drug Delivery Properties. World Molecular Engineering Congress 2016, New York

Abhignyan Nagesetti, George Dulikravich, Anthony J McGoron: Silica Nanoparticle Transport in Simulated Tumor Microenvironments: The Role of Surface Functionalization and Cellular Autophagy. Biomedical Engineering Society, Tampa, Florida 2015.

Abhignyan Nagesetti, Diego Estumano, Helcio R. B. Orlande, Marcelo J. Colaço, George Dulikravich, Anthony McGoron: Influence of Surface Modification and Temperature on Silica Nanoparticle Transport in Mono-layer and Tumor Spheroid Cultures. Society for personalized nanomedicine, Florida International University $2015 *$ (Junior investigator award)

Abhignyan Nagesetti, Diego Estumano, Helcio R. B. Orlande, Marcelo J. Colaço, George Dulikravich, Anthony McGoron: The Effect of Surface Functionalization and Temperature on Nanoparticle Penetration into Tumor Spheroids. Biomedical Engineering Society, San Antonio, Texas 2014

Abhignyan Nagesetti, George Dulikravich, Anthony McGoron: Enhancing Nanoparticle penetration in tumors via the use of Hyperthermia: An experimental and theoretical investigation. BCI Conference MARC FIU, 2014

Abhignyan Nagesetti, Anthony McGoron: The Effect of Hyperthermia on Penetration of Nanoparticles in Extracellular Matrix Gels and Tumor Spheroids. Biomedical Engineering Society, Atlanta, Georgia, 2012

Abhignyan Nagesetti, Anthony McGoron:Effect of Hyperthermia on the cytotoxicity of Doxorubicin and penetration of nanoparticles in a tumor spheroid model, NanoBio, University of South Florida, Tampa,2012. 


\section{SPRINGER LICENSE TERMS AND CONDITIONS}

May 12, 2017

This Agreement between Abhignyan Nagesetti ("You") and Springer ("Springer") consists of your license details and the terms and conditions provided by Springer and Copyright Clearance Center.

License Number

License date

Licensed Content Publisher

Licensed Content Publication

Licensed Content Title

Licensed Content Author

Licensed Content Date

Licensed Content Volume

Licensed Content Issue

Type of Use

Portion

Number of

figures/tables/illustrations

Author of this Springer article No

Order reference number

Original figure numbers

Title of your thesis /

dissertation

Expected completion date

Estimated size(pages)

Requestor Location

Billing Type

Billing Address

Total

Terms and Conditions

1

1
4106611026283

Springer

Cell and Tissue Research

High-resolution deep imaging of live cellular spheroids with lightsheet-based fluorescence microscopy

Francesco Pampaloni

Jan 1,2013

352

Thesis/Dissertation

Figures/tables/illustrations

Figure 1 a

THE EFFECT OF HYPERTHERMIA ON DOXORUBICIN THERAPY AND NANOPARTICLE PENETRATION IN MULTICELLULAR OVARIAN CANCER SPHEROIDS

May 2017

178

Abhignyan Nagesetti

9429 Sw 4th Lane

MIAMI, FL 33174

United States

Attn: Abhignyan Nagesetti

Invoice

Abhignyan Nagesetti

9429 Sw 4th Lane

MIAMI, FL 33174

United States

Attn: Abhignyan Nagesetti

0.00 USD 
Introduction

The publisher for this copyrighted material is Springer. By clicking "accept" in connection with completing this licensing transaction, you agree that the following terms and conditions apply to this transaction (along with the Billing and Payment terms and conditions established by Copyright Clearance Center, Inc. ("CCC"), at the time that you opened your Rightslink account and that are available at any time at http://myaccount.copyright.com). Limited License

With reference to your request to reuse material on which Springer controls the copyright, permission is granted for the use indicated in your enquiry under the following conditions: - Licenses are for one-time use only with a maximum distribution equal to the number stated in your request.

- Springer material represents original material which does not carry references to other sources. If the material in question appears with a credit to another source, this permission is not valid and authorization has to be obtained from the original copyright holder.

- This permission

- is non-exclusive

- is only valid if no personal rights, trademarks, or competitive products are infringed.

- explicitly excludes the right for derivatives.

- Springer does not supply original artwork or content.

- According to the format which you have selected, the following conditions apply accordingly:

- Print and Electronic: This License include use in electronic form provided it is password protected, on intranet, or CD-Rom/DVD or E-book/E-journal. It may not be republished in electronic open access.

- Print: This License excludes use in electronic form.

- Electronic: This License only pertains to use in electronic form provided it is password protected, on intranet, or CD-Rom/DVD or E-book/E-journal. It may not be republished in electronic open access.

For any electronic use not mentioned, please contact Springer at permissions.springer@spiglobal.com.

- Although Springer controls the copyright to the material and is entitled to negotiate on rights, this license is only valid subject to courtesy information to the author (address is given in the article/chapter).

- If you are an STM Signatory or your work will be published by an STM Signatory and you are requesting to reuse figures/tables/illustrations or single text extracts, permission is granted according to STM Permissions Guidelines: http://www.stm-assoc.org/permissionsguidelines/

For any electronic use not mentioned in the Guidelines, please contact Springer at permissions.springer@spi-global.com. If you request to reuse more content than stipulated in the STM Permissions Guidelines, you will be charged a permission fee for the excess content.

Permission is valid upon payment of the fee as indicated in the licensing process. If permission is granted free of charge on this occasion, that does not prejudice any rights we might have to charge for reproduction of our copyrighted material in the future.

-If your request is for reuse in a Thesis, permission is granted free of charge under the following conditions:

This license is valid for one-time use only for the purpose of defending your thesis and with a maximum of 100 extra copies in paper. If the thesis is going to be published, permission needs to be reobtained.

- includes use in an electronic form, provided it is an author-created version of the thesis on his/her own website and his/her university's repository, including UMI (according to the definition on the Sherpa website: http://www.sherpa.ac.uk/romeo/);

- is subject to courtesy information to the co-author or corresponding author. 
Geographic Rights: Scope

Licenses may be exercised anywhere in the world.

Altering/Modifying Material: Not Permitted

Figures, tables, and illustrations may be altered minimally to serve your work. You may not

alter or modify text in any manner. Abbreviations, additions, deletions and/or any other

alterations shall be made only with prior written authorization of the author(s).

Reservation of Rights

Springer reserves all rights not specifically granted in the combination of (i) the license

details provided by you and accepted in the course of this licensing transaction and (ii) these terms and conditions and (iii) CCC's Billing and Payment terms and conditions.

License Contingent on Payment

While you may exercise the rights licensed immediately upon issuance of the license at the end of the licensing process for the transaction, provided that you have disclosed complete and accurate details of your proposed use, no license is finally effective unless and until full payment is received from you (either by Springer or by CCC) as provided in CCC's Billing and Payment terms and conditions. If full payment is not received by the date due, then any license preliminarily granted shall be deemed automatically revoked and shall be void as if never granted. Further, in the event that you breach any of these terms and conditions or any of CCC's Billing and Payment terms and conditions, the license is automatically revoked and shall be void as if never granted. Use of materials as described in a revoked license, as well as any use of the materials beyond the scope of an unrevoked license, may constitute copyright infringement and Springer reserves the right to take any and all action to protect its copyright in the materials.

Copyright Notice: Disclaimer

You must include the following copyright and permission notice in connection with any reproduction of the licensed material:

"Springer book/journal title, chapter/article title, volume, year of publication, page, name(s) of author(s), (original copyright notice as given in the publication in which the material was originally published) "With permission of Springer"

In case of use of a graph or illustration, the caption of the graph or illustration must be included, as it is indicated in the original publication.

Warranties: None

Springer makes no representations or warranties with respect to the licensed material and adopts on its own behalf the limitations and disclaimers established by CCC on its behalf in its Billing and Payment terms and conditions for this licensing transaction.

Indemnity

You hereby indemnify and agree to hold harmless Springer and CCC, and their respective officers, directors, employees and agents, from and against any and all claims arising out of your use of the licensed material other than as specifically authorized pursuant to this license.

No Transfer of License

This license is personal to you and may not be sublicensed, assigned, or transferred by you without Springer's written permission.

No Amendment Except in Writing

This license may not be amended except in a writing signed by both parties (or, in the case of Springer, by CCC on Springer's behalf).

Objection to Contrary Terms

Springer hereby objects to any terms contained in any purchase order, acknowledgment, check endorsement or other writing prepared by you, which terms are inconsistent with these terms and conditions or CCC's Billing and Payment terms and conditions. These terms and conditions, together with CCC's Billing and Payment terms and conditions (which are incorporated herein), comprise the entire agreement between you and Springer (and CCC) concerning this licensing transaction. In the event of any conflict between your obligations 
established by these terms and conditions and those established by CCC's Billing and Payment terms and conditions, these terms and conditions shall control. Jurisdiction

All disputes that may arise in connection with this present License, or the breach thereof, shall be settled exclusively by arbitration, to be held in the Federal Republic of Germany, in accordance with German law.

Other conditions:

V 12AUG2015

Questions? customercare@copyright.com or +1-855-239-3415 (toll free in the US) or +1-978-646-2777. 